\title{
Statics and dynamics of solvent-free models for liquid bilayer membranes
}

\author{
Dissertation zur Erlangung des \\ mathematisch-naturwissenschaftlichen Doktorgrades \\ „Doctor rerum naturalium“ \\ der Georg-August-Universität Göttingen
}

\author{
vorgelegt von \\ Martin Hömberg \\ aus Wesel am Rhein
}

Göttingen 2011 
D 7

Referent: Prof. Dr. Marcus Müller

Koreferentin: Prof. Dr. Annette Zippelius

Weitere Referentin: Prof. Dr. Friederike Schmid

Tag der mündlichen Prüfung: 


\section{Danksagung}

Diese Dissertation ist in den Jahren 2007 bis 2011 im Institut für theoretische Physik an der Universität Göttingen entstanden. Auch wenn es die Arbeit eines Einzelnen ist, so wäre sie niemals ohne die Mithilfe von vielen Anderen möglich gewesen.

Ich danke meinem Betreuer und Doktorvater Prof. Marcus Müller für die Ausgabe eines derartig vielseitigen und abwechselungsreichen Themas, für die herausragende fachliche Betreuung und die langjährige, sehr gute Zusammenarbeit. Zu großem Dank verpflichtet bin ich Kostas Daoulas, der einen Großteil unseres Modells mitentwickelt hat und mir mit vielen Erklärungen zur Seite stand. Gedankt sei auch Fabien Léonforte, Claudio Pastorino und Cem Servantie, die mir bei zahlreichen physikalischen und technischen Fragen den richtigen Weg gewiesen haben. Für hilfreiche Kommentare möchte ich mich weiterhin bei Veronica Chappa, Sandra Frank, Marc Fuhrmans, André Galuschko, Giovanni Marelli, David Morse, Pablo Nigra, Yuki Norizoe, Julian Shillcock und Annette Zippelius bedanken. Einen unschätzbaren Beitrag zu dieser Dissertation haben Birger Steinmüller und Stefan Schneider geleistet, die diese Arbeit gründlich gelesen und unzählige Verbesserungen eingebracht haben.

Finanzielle Untersützung kam von der Volkswagenstiftung, der DFG im Rahmen des SFB 937, Teilprojekt A7 und des DAADs, der einen Austausch mit Buenos Aires, Argentinien ermöglicht hat. Dank geht auch an die GWDG, das HLRN, sowie das Jülich Supercomputing Centre, die etliche CPU-Stunden an Rechenzeit zur Verfügung gestellt haben.

$\mathrm{Zu}$ guter letzt möchte ich mich bei Petra und meiner Freundin Dominika dafür bedanken, dass sie mich gelegentlich auch an ein Leben außerhalb der Physik erinnert haben. 



\section{Zusammenfassung}

Kollektive Phänomene in Lipidmembranen, wie die Porenbildung und die Fusion, die Selbstorganisation zu Doppelschichten oder die laterale Entmischung mehrerer Lipidsorten haben in den letzten Jahren großes Interesse geweckt. All diese Phänomene eint die Tatsache, dass sie auf mesoskopischen Skalen, also Mikrometern und Mikrosekunden, stattfinden. Diese Skalen sind zu klein um in Experimenten direkt beobachtet werden zu können, sie sind aber auf Grund der Vielzahl der beteiligten Freiheitsgrade zu groß für Computersimulationen mit atomarer Auflösung. Vergröberte Modelle verfügen über erheblich weniger Freiheitsgrade und weichere Potentiale und gestatten eine qualitative Untersuchung dieser Phänomene mittels Computersimulationen.

Im Rahmen dieser Arbeit wird ein vergröbertes, lösungsmittelfreies Modell für die Simulation von Doppelschichten amphiphiler Moleküle präsentiert und eingehend untersucht. Die Moleküle werden durch lineare Ketten von Punktteilchen beschrieben, die über Federn miteinander verbunden sind. Die ungebundenen Wechselwirkungen entstammen einem klassischen, gewichteten Dichtefunktional für die Freie Energie, das eine Entwicklung bis zur dritten Ordnung in gewichteten molekularen Dichten darstellt. Die auftretenden Entwicklungskoeffizienten und Wichtungsfunktionen ermöglichen es, im Rahmen einer Mean-Field-Näherung die Zustandsgleichung und die lokale Flüssigkeitsstruktur unabhängig voneinander vorzugeben. Wir verwenden Dissipative Particle Dynamics (DPD) Simulationen um die Eigenschaften des Modells numerisch zu untersuchen.

Wir untersuchen die Selbstorganisation zu kondensierten Morphologien, das Phasendiagramm von Doppelschichten sowie strukturelle und mechanische Eigenschaften der flüssigen Phase $\left(L_{\alpha}\right)$ und einer Gelphase $\left(L_{\beta}\right)$, darunter den statischen Strukturfaktor, die Biegesteifigkeit und die Flächenkompressibilität. Es werden vier Verfahren zur präzisen Lokalisierung des Hauptphasenübergangs $\left(L_{\beta} \leftrightarrow L_{\alpha}\right)$ aufgezeigt und die Freie Energie wird als Funktion eines Konformations-Ordnungsparameters berechnet. Die Linienspannung zwischen beiden Phasen wird über das Fluktuationsspektrum der Kontaktlinie und aus der Höhe der freien Energie-Barriere berechnet und verglichen.

Weiterhin untersuchen wir die Brown'sche Bewegung einzelner Moleküle, sowie die kollektive Bewegung von Molekülclustern anhand der mittleren quadratischen Verschiebung, der Geschwindigkeits-Autokorrelationsfunktion und des dynamischen Strukturfaktors. Kollektive Diffusion ist nur für sehr kurze 
Zeiten sichtbar; es existieren keine ausgedehnten Strömungsfelder. Wir untersuchen die Oberflächenviskosität und die Intermonolagenreibung mit jeweils zwei verschiedenen Methoden. Für die Berechnung der Intermonolagenreibung leiten wir eine neue Green-Kubo-Relation ab und präsentieren eine modifizierte Variante der Seifert-Langer-Theorie, die die Dynamik von Undulationsmoden in einem lösungsmittelfreien Modell beschreibt. Schließlich bilden wir unser Modell auf ein noch einfacheres Scheibenmodell ab, in dem alle intramolekularen Freiheitsgrade eliminiert sind, und untersuchen ob es möglich ist, die ausintegrierte Reibung der intramolekularen Freiheitsgrade einzig durch den DPD-Thermostaten zu modellieren. 


\section{Abstract}

Collective phenomena in lipid membranes, like pore formation and fusion, selfassembly, or lateral demixing of lipid mixtures, have attracted tremendous interest over the last years. Often, collective phenomena involve mesoscopic time and length scales, microseconds and micrometers, which are difficult to observe directly in experiments and which are at present beyond the scales that can be addressed by models with atomistic resolution. The reduced number of degrees of freedom and the softer potentials in coarse-grained models open up the opportunity to address the mesoscopic scales computationally and to gain qualitative insights.

In this work we present a solvent-free, coarse-grained model for amphiphilic bilayers. The molecules are represented by linear bead-spring chains and the non-bonded interactions are derived from a classical density functional, which is an expansion of the free energy in terms of weighted molecular densities up to third order. Within the mean-field approximation the involved expansion coefficients and weighting functions can be utilized to tune the equation of state and the local, fluid structure of the model independently. We employ Dissipative Particle Dynamics (DPD) simulations to study the properties of the model numerically.

We study the self-assembly process of various morphologies, the bilayer phase diagram, and structural and mechanical properties of the fluid phase $\left(L_{\alpha}\right)$ and a gel phase $\left(L_{\beta}\right)$, e.g., the static structure factor, the bending rigidity, and the area compressibility. Three different methods for precisely locating the point of the main phase transition $\left(L_{\beta} \leftrightarrow L_{\alpha}\right)$ are presented and the free energy as a function of a conformational order parameter is computed. We estimate the line tension between fluid and gel domains from the power spectrum of the interface fluctuations, as well as from the height of the free energy barrier.

The Brownian motion of individual molecules and the collective motion of molecular clusters are investigated by looking at the mean-square displacement, the velocity autocorrelation function, and the intermediate scattering function. Collective diffusion exists only for very short time intervals and no flow pattern is observed. The surface viscosity and the intermonolayer friction are each measured by two different methods. To this end we derive a new Green-Kubo relation for computing the intermonolayer friction and present a modified version of the Seifert-Langer-theory that is able to describe the dynamics of undulation modes in a solvent-free model. Finally, we map our 
bead-spring model onto a two-dimensional model of soft discs, where all intramolecular degrees of freedom are integrated out. We study the question if it is possible to obtain the same dissipation mechanisms as before by using only a DPD thermostat. 


\section{Contents}

1 Introduction 1

2 Coarse-grained model $\quad 13$

2.1 Atomistic and coarse-grained models - an overview . . . . . . 13

2.2 Model description I: physical part . . . . . . . . . . . . . 17

2.2.1 Bonded interactions . . . . . . . . . . . . . . 17

2.2 .2 Non-bonded interactions . . . . . . . . . . . . . 18

2.3 Model description II: computational part . . . . . . . . . . 26

2.3 .1 Forcefield . . . . . . . . . . . . . . . 26

2.3.2 Simulation in various ensembles . . . . . . . . . . . 29

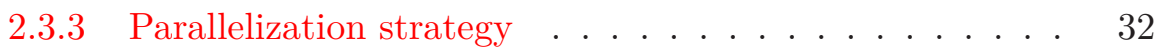

3 Static properties $\quad 37$

3.1 Phase diagram and phase properties . . . . . . . . . . 38

3.1 .1 Self-assembly . . . . . . . . . . . . . . . . . 39

3.1 .2 Phase diagram . . . . . . . . . . . . . . . . . 42

3.1 .3 Observables . . . . . . . . . . . . . . . . . . . . 43

3.1 .4 Phase properties . . . . . . . . . . . . . . . . . . . 49

3.1 .5 Summary . . . . . . . . . . . . . . 54

3.2 Main phase transition . . . . . . . . . . . . 55

3.2 .1 Free energy calculations . . . . . . . . . . . . 57

3.2 .2 Location of the transition point . . . . . . . . . . 61

3.2 .3 Discussion and conclusion . . . . . . . . . . 66

3.3 Line tension and bilayers in the miscibility gap . . . . . . . 67

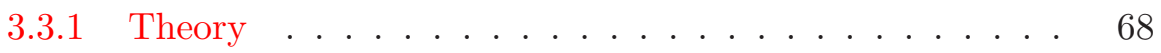

3.3 .2 Results and discussion . . . . . . . . . . . . . . 71

3.3 .3 Conclusions . . . . . . . . . . . . . 76

4 Dynamic properties $\quad 77$

4.1 Brownian vs. correlated motion . . . . . . . . . . 78

4.1 .1 Methods . . . . . . . . . . . . . . . 80

4.1 .2 Results I: Brownian motion . . . . . . . . . . . 85

4.1.3 Results II: correlated motion . . . . . . . . . . . . 90

4.1 .4 Conclusions . . . . . . . . . . . . . . . . . . . 99 
4.2 Surface viscosity and intermonolayer friction . . . . . . . . 100 4.2 .1 Theory . . . . . . . . . . . . . . . . 102

4.2.2 Results and discussion . . . . . . . . . . . . . 110

4.2 .3 Conclusions . . . . . . . . . . . . . . . . . 122

4.3 Mapping to a fluid of soft discs . . . . . . . . . . . 123

4.3 .1 Interactions . . . . . . . . . . . . . . 126

4.3.2 Mapping of the statics . . . . . . . . . . . . . . 132

4.3.3 Mapping of the dynamics . . . . . . . . . . . . 138

4.3 .4 Conclusions . . . . . . . . . . . . . . . . . 143

5 Conclusions and outlook $\quad 145$

$\begin{array}{ll}\text { A Interaction coefficients } & 149\end{array}$

$\begin{array}{ll}\text { Bibliography } & 151\end{array}$ 


\section{List of Figures}

1.1 Schematic fusion pathway through hemifusion . . . . . . . . 3

1.2 Microscopy picture of a vesicle at phase coexistence . . . . . . 4

1.3 Dynamical states of a vesicle . . . . . . . . . . 5

1.4 Atomistic and systematically coarse-grained models . . . . . . 8

2.1 Lipid architecture . . . . . . . . . . . . . . . . . . 18

2.2 Mean-field equation of state of heads and tails . . . . . . . . . . 21

2.3 The weighting functions $w_{2}(r)$ and $w_{3}(r) \ldots \ldots \ldots . \ldots 24$

2.4 Sketch of the force-decomposition parallelization . . . . . . 33

2.5 Scaling plot of the simulation code . . . . . . . . . 36

3.1 Self-assembly to a bilayer membrane . . . . . . . . . . . 40

3.2 Phase diagram . . . . . . . . . . . . . . . . . . . . 43

3.3 Images of the four phases . . . . . . . . . . . . . . 44

3.4 Static structure factor of the $L_{\alpha}$ and $L_{\beta}$ phases . . . . . . . 50

3.5 Lateral pair correlation function of the beads . . . . . . . . 51

3.6 Density profile of the $L_{\alpha}$ and the $L_{\beta}$ phases . . . . . . . . 52

3.7 Undulation power spectrum of the fluid phase . . . . . . . . 54

3.8 A fluid domain in the gel phase . . . . . . . . . . . 57

3.9 Hysteresis loops . . . . . . . . . . . . . . . . . . . . 62

3.10 Free energy perturbation calculation . . . . . . . . . 63

$3.11 F(\Delta S)$ at phase coexistence . . . . . . . . . . 64

3.12 Specific heat, $C$, and total energy, $\langle U\rangle \ldots \ldots \ldots \ldots$

3.13 Definition of the Gibbs dividing surface . . . . . . . . . 72

3.14 Bilayer configurations in the miscibility gap . . . . . . . 73

3.15 Free energy profile at phase coexistence . . . . . . . . . 74

3.16 Power spectrum of the boundary line fluctuations . . . . . . 75

4.1 Lateral diffusion of several lipids . . . . . . . . . . . . . 79

4.2 Lateral mean square displacement of the lipids . . . . . . . 86

4.3 Velocity autocorrelation function of the lipids . . . . . . . . 88

4.4 Dynamic structure factor of the fluid phase . . . . . . . . . 92

4.5 Dynamic structure factor of the gel phase . . . . . . . . . 94

4.6 Structural relaxation of the fluid phase . . . . . . . . . . 96

4.7 Flow motion of large lipid patches . . . . . . . . . . 98 
4.8 The two shear modes in bilayer membranes . . . . . . . . . . 102

4.9 Sketch for the Seifert-Langer theory . . . . . . . . . . 106

4.10 Green-Kubo measurement of the surface viscosity . . . . . . . . 111

4.11 RNEMD velocity, temperature, and density profile . . . . . . 112

4.12 Diffusion and viscosity with the TDPD thermostat . . . . . . . 115

4.13 Intermonolayer force autocorrelation and its integral . . . . . . 117

4.14 Undulation autocorrelation in the time domain . . . . . . . . 118

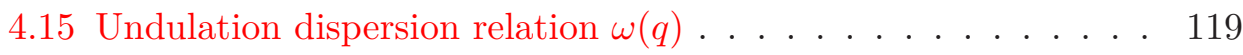

4.16 Widths of the Rayleigh and the Brillouin lines . . . . . . . . 120

4.17 Mapping from lipids to soft discs . . . . . . . . . . . . . 124

4.18 Interaction potentials of the LRM . . . . . . . . . . . 133

4.19 Lateral pair correlation function of the LRM . . . . . . . 136

4.20 Comparison of $G(q)$ between the HRM and the LRM . . . . . 137

4.21 Universality of the LRM's diffusion coefficient . . . . . . . . . 140

4.22 Intermonolayer friction of the LRM . . . . . . . . . . . . 141

4.23 Surface viscosity of the LRM . . . . . . . . . . . . . 142 


\section{List of Tables}

3.1 Self-assembled morphologies . . . . . . . . . . . . . . . 42

3.2 Compilation of important static properties . . . . . . . . 55

4.1 Surface viscosities and intermonolayer friction coefficients . . . 112

4.2 Pressure of the LRM with the scaled attractions . . . . . . . 134

4.3 DPD parameters for studying the LRM's dynamics . . . . . . 139

A.1 Frequently occurring interaction parameters . . . . . . . . 149

A.2 Intramolecular interactions for $N=10 \ldots \ldots \ldots \ldots$

A.3 Intramolecular interactions for $N=16 \ldots \ldots \ldots$ 



\section{List of Abbreviations}

Lipid polar head groups

PC phosphatidylcholine

PE phosphatethanolamine

\section{Lipids}

DLPC di-laureoyl PC

DMPC di-myristoyl PC

DOPC di-oleoyl PC

DPPC di-palmitoyl PC

DPPE di-palmitoyl PE

\section{Technical terms}

DFT density functional theory

DPD dissipative particle dynamics

EOS equation of state

FCS fluorescence correlation spectroscopy

FD force decomposition

FEP free energy perturbation theory

FFT fast Fourier transform

FRAP fluorescence recovery after photobleaching

FWHM full width at half maximum

GK Green-Kubo

HRM high resolution model

ISF intermediate scattering function

LRM low resolution model

MC Monte Carlo

MD molecular dynamics

MDPD multi-body DPD

MSD mean-square displacement

NEMD non-equilibrium MD

NGP non-Gaussianity parameter

OZ Ornstein-Zernicke

PME particle-mesh Ewald

QENS quasi-elastic neutron scattering

RNEMD reverse NEMD 
SL Seifert-Langer

TDPD transverse DPD

US umbrella sampling

VACF velocity autocorrelation function

WCA Weeks-Chandler-Andersen

WHAM weighted histogram analysis method 


\title{
1 Introduction
}

\author{
"Nature is made in such a way as to be able to \\ be understood. Or perhaps I should put it -more \\ correctly- the other way around, and say that we \\ are made in such a way as to be able to \\ understand Nature."
}

(Werner Heisenberg)

In contrast to the widely held belief that fats are something vicious, which endanger our health and should be avoided at all cost, only few people are aware of the true relevance of these molecules for our body. Fats are essential ingredients of our diet, they provide for tasty meals, good health, and longevity, and enable our body to produce important substances like sex hormones, vitamin $\mathrm{D}$, or bile (the liquid in our stomach to break down the food). They constitute a major part of our brain and are among the most abundant molecules in our body $[1,2]$.

In science, fats are called lipids. They often appear in combination with other fundamental building blocks of cell biology, like carbohydrates, genes, or proteins. While proteins are the molecular machines in our body, which perform highly specific tasks, and genes encode the way how these proteins are catalyzed, lipids seem to be more general and less specific. This is certainly related to their less intriguing chemical structure, which only comprises a polar head group favoring the contact with water ("hydrophilic") and one or two nonpolar tails avoiding such a contact ("hydrophobic"). Although there are many kinds of lipids which differ in chemical details, like the degree of saturation of the carbon tails or the residues in the head groups, they are all qualitatively similar and the study of individual molecules does not offer much insight into the relevance of these details or into the lipids' function.

Their key property becomes visible when they are placed in water. Due to their amphiphilic nature, i.e., they like and dislike water at the same time, they self-assemble into extended morphologies, e.g., micelles, bilayers, or vesicles, which minimize the contact area of the hydrophobic tails with the water [3] and whose size varies between $10 \mathrm{~nm}$ and $10 \mu \mathrm{m}$. Bilayers are thin films consisting of two stacked layers of apposing lipid molecules with the head groups pointing outwards, i.e., towards the water, and the tails pointing inwards (see Fig. 1.1i). They are of particular importance in biology, because they constitute the cell membrane as well as the membranes of cell organelles. There, 
they are often decorated with proteins or carbohydrates and are then called biomembranes. Vesicles are spherically closed bilayers, which transport small molecules or liquids in cells, and are also referred to as liposomes.

The lipids in these self-assembled aggregates attract each other through weak van-der-Waals interactions and typical forces are on the order of magnitude of $10 \mathrm{pN}$. A comparison of these forces with those arising from thermal agitation can be achieved by rewriting the thermal energy, $k_{B} T$, as a product of a typical molecular separation, $1 \mathrm{~nm}$, and a thermal force,

$$
\text { thermal energy }=1 k_{B} T \approx 4 \mathrm{pN} \times 1 \mathrm{~nm} .
$$

Here, $k_{B}$ is Boltzmann's constant, which is a universal number, and $T=$ $293 \mathrm{~K}=20^{\circ} \mathrm{C}$ is the room temperature. We find that the thermal forces are on the same order of magnitude and, thus, they cannot be neglected. The coincidence of these two energy scales leads to important thermal fluctuations of these aggregates and to a permanently changing shape.

Besides lipids, this work also deals with another class of molecules: polymers. These are synthetic macromolecules consisting of a large number of simple repeat units which are covalently bound to form long molecules. If more than one type of repeat units is used to grow a polymer, the resulting molecule is called a copolymer for which an endless number of possible molecular compositions and structures is available. If the different types are arranged in two blocks, the polymers are called diblock copolymers; if they are arranged in three blocks, triblock copolymers.

Many block copolymers self-assemble in a selective solvent into a range of different morphologies, like spheres, rods, vesicles, or large micelles [4, 5]. There is a huge interest in polymeric vesicles, "polymersomes", because they are in many respects comparable to lipid vesicles, but at the same time they offer several advantages. Due to the thicker shell they are generally tougher, the physical and chemical properties can be tailored over a wide range by attaching various side groups to the polymers, and they can be cross-linked after the preparation to capsules. This cross-linking makes the polymersomes stable for long times and leads to very different elastic properties, because these capsules are not fluid anymore. These abilities are interesting for many practical applications, like the potential use as microreactors, drug-delivery vehicles, or simple model systems of biomembranes. Polymer vesicles are also able to encapsulate proteins, nano-particles, or other biologically active molecules $[6,7$, and references therein].

The self-assembly is just one example of a collective phenomenon of lipids and polymers; the fusion of biomembranes is another one. It is a crucial step in many biological processes, like intracellular traffic, viral infection, fertilization, or the transport of small molecules through a membrane [1]. Although a large 


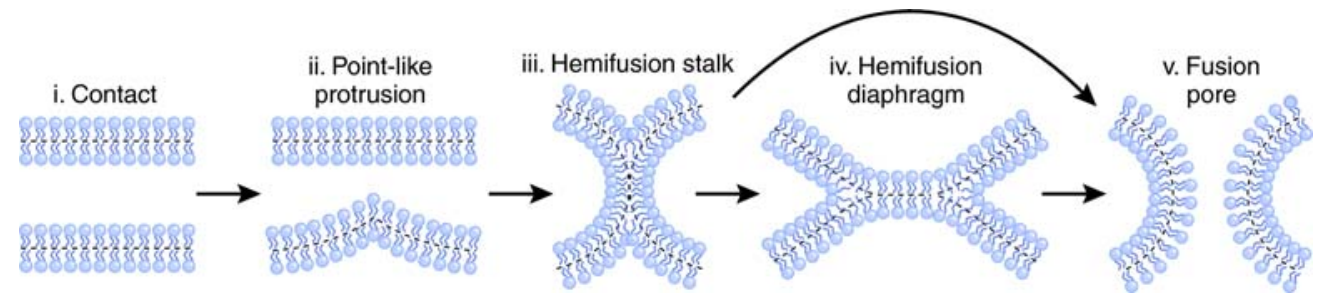

Figure 1.1: Schematic fusion pathway: (i) Pre-fusion contact. (ii) A point-like membrane protrusion minimizes the energy of the hydration repulsion between the proximal leaflets of the membranes coming into immediate contact. (iii) A hemifusion stalk with proximal leaflets fused and distal leaflets unfused. (iv) Stalk expansion yields the hemifusion diaphragm. (v) A fusion pore forms either in the hemifusion diaphragm bilayer or directly from the stalk. Dashed lines show the boundaries of the hydrophobic surfaces of monolayers. Figure and caption taken from Ref. 8.

fraction of the literature discusses the role fusion proteins play, it seems that their main purpose is to bring two membranes into close apposition and to create stress. Once this stage has been reached, there is growing evidence that the subsequent process, i.e., the rupture of the bilayers and the formation of intermediate structures, which finally create a fusion pore (see Fig. 1.1), results from the lipids' inherent properties [8-12]. Consequently, it should be possible to study the fusion of lipid bilayers even in simple models without any proteins. A typical fusion pore spans a few nanometers in diameter and a typical fusion event takes several microseconds [13].

Similar to lipid vesicles, polymer vesicles can fuse or create pores, too. The fusion rates of diblock and triblock copolymer vesicles were studied experimentally as functions of the water concentration, the polymer concentration, or the block length [14-16]. Due to the higher molecular weight, these fusion events involve significant longer times, e.g., seconds to minutes [15].

The lateral phase separation of lipid mixtures, which naturally occur in biomembranes, has attracted abiding interest in recent years, and is yet another collective phenomenon. Under certain conditions, unlike lipid species demix and form domains of like species [18, 19, and references therein]. These domains, "rafts", cause a lateral heterogeneity of the bilayer which is deemed to play an important role in the organization of cell membranes. A typical domain size is on the order of $10 \mathrm{~nm}$ and contains hundreds of lipids. A quantification of this lateral heterogeneity has proven difficult, therefore simpler systems, with only one or two kinds of lipids and often some cholesterol [17], are studied (see Fig. 1.2). Although a large number of phase diagrams for such systems have been studied [20, and references therein], it is difficult to get a comprehensive overview from these two-dimensional projections of a much large parameter space. 

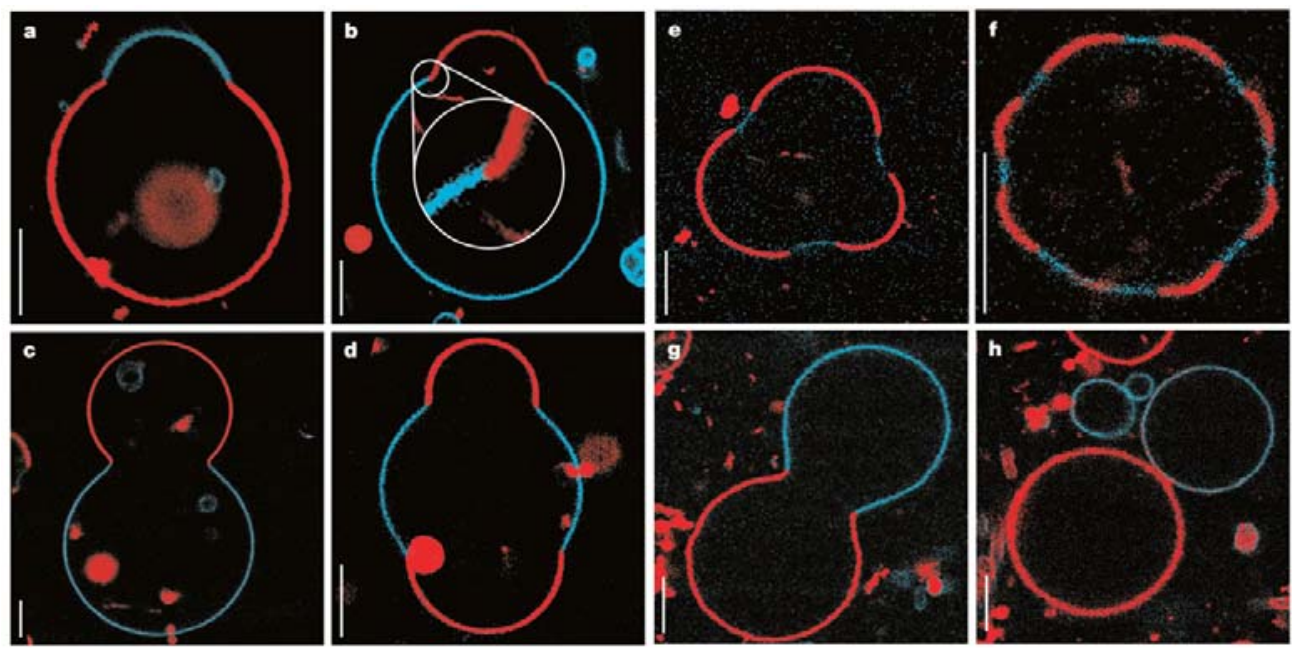

Figure 1.2: Two photon microscopy picture of giant unilamellar vesicles formed from a ternary mixture of sphingomyelin, DOPC, and cholesterol at various compositions. The molecules phase separate into a liquid-ordered phase (blue) and a liquid-disordered phase (red). The scale bar is $5 \mu \mathrm{m}$. Reprinted by permission from Macmillan Publishers Ltd: Nature [17], copyright 2003.

Inspired by this heterogeneity, chemists have recently started to tailor polymersomes composed of incompatible macromolecules [21-23] or even "lipopolymersomes" which are mixtures of lipids and polymers [24]. In contrast to the biomembranes, the exact chemical composition of these multi-component polymersomes is known, so that it is possible to study the demixing behavior quantitatively. These objects become particularly interesting if polymers of different chemical or physical properties are combined into a single polymersome.

A final example comes from the rheology of biofluids. The flow resistance of blood in microvessels is largely determined by the deformability of the red blood cells. It is known that diseases such as diabetes mellitus and sickle cell anemia decrease this deformability and thereby enhance the flow resistance of blood which causes, in turn, other diseases [25]. It is therefore of great interest to understand the cell's elasticity in shear flow. Red blood cells are, however, very complex objects, due to their cytoskeleton and their biconcave shape, so that simpler single-component vesicles, with a size of 10 micrometers or larger, are preferred in most studies.

Such vesicles under shear flow have been studied in experiments, analytical calculations, and computer simulations [26, 27, and references therein]. Three different kinds of motion are observed: the tank-treading, the tumbling, and the trembling motion (see Fig. 1.3). In the tank-treading motion, the vesicle 


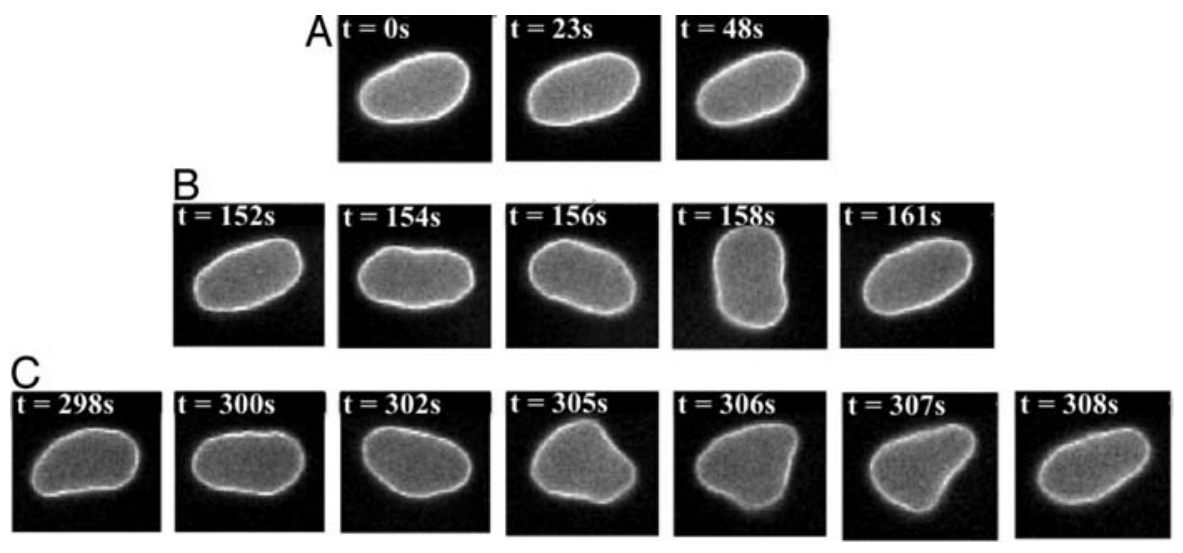

Figure 1.3: Dynamical states of a vesicle with mean radius $R=6.19 \mu \mathrm{m}$, at different values of the fluid's vorticity and different strain rates. A: tank-treading, B: tumbling, C: trembling motion. Taken from Ref. 26.

keeps a constant inclination angle between its major axis and the flow direction, while its membrane rotates about the internal fluid. In the tumbling motion the entire vesicle rotates and in the trembling motion oscillations of the inclination angle around $0^{\circ}$ with excursions smaller than $\pi / 2$ occur [26]. Typical oscillation periods are milliseconds to seconds.

Although the tank-treading and the tumbling motion can be explained by chiefly considering the viscosity of the fluids on the inside and the outside [28], the surface viscosity and the intermonolayer friction of the membrane also contribute to the energy dissipation. The effect of the monolayer's surface viscosity has been studied to some extent [29, 30], but the role of the intermonolayer friction [31, 32] has received less attention, because the molecular character of the bilayer must be taken into account. Such a study has not yet been feasible due to the widely separated length scales, micrometers for the vesicles and nanometers for the molecules.

Not only lipid vesicles, but also polymersomes under shear flow are of interest. Recently, a group succeeded in creating leuko-polymersomes that act like leukocytes, but are made of polymers, and can bind to inflammations [33, 34]. Of course, such polymersomes are exposed to the same fast flow in the blood vessels as any other object, thus the interplay of the elastic and the dissipative properties of polymersomes under shear has to be known before such objects can be used in medical applications.

The aforementioned examples show that the properties of lipids manifest themselves in the collective behavior where hundreds or thousands of molecules cooperate. It is exactly this cooperative nature which makes the study of collective phenomena so interesting on the one hand, and so challenging on the other 
hand. It is inadequate to study individual molecules only, but large numbers of them must be taken into account to see how their chemical details translate into a mesoscopic structure. Even though polymers and lipids differ a great deal in their chemistry and their characteristic scales, they expose the same qualitative behavior. Thus, there are many good reasons to study collective phenomena in lipid bilayers together with those in polymeric membranes and to explore similarities and differences in their behavior.

\section{Coarse-grained models}

A direct observation of such collective phenomena in experiments is often difficult or ambiguous, so that computer simulations are frequently employed to acquire a better understanding of the experimental results. Simulations offer several advantages, for instance it is possible to repeat the same computer experiments many times under exactly known conditions, e.g., temperature, pressure, $\mathrm{pH}$, or molecular composition, or to study the effect of changing one of these parameters precisely. Since the phenomena in membranes evolve on vastly different time and length scales, many different models and computational techniques have been devised for studying specific questions. They can be roughly categorized into atomistic, coarse-grained, and continuum models.

In atomistic models one tries to describe every component of a membrane embedded in a solvent with the greatest possible accuracy, so that a chemically realistic model is obtained. Specifically, the covalent bonds are often taken into account by rigid constraints between the atoms [35-37] and are supplemented by carefully determined torsional and dihedral angle potentials. Non-bonded interactions, i.e., the short-ranged van-der-Waals and the long-ranged Coulomb interactions are treated explicitly, the former ones by empirically determined pairwise interaction potentials, and the latter ones by Ewald summation or particle-mesh Ewald methodologies [38, 39]. Thus, atomistic simulations crucially depend on the existence of previously determined, accurately calibrated force fields for all possible bonded and non-bonded interactions [40-43]. Most of these simulations are performed by a technique called molecular dynamics (MD) where the positions and velocities of all atoms are known in an initial state. The momentary forces between all atoms are evaluated, Newton's equations of motion are integrated for a short time step $(\sim 1 \mathrm{fs})$, and new positions and velocities are obtained. Then the process starts all over [44-46].

Atomistic models are very well suited for the study of small and specific systems where the chemical details of the constituents matter, e.g., in protein folding [47, 48], lipid-protein or lipid-DNA interactions [49], or in the transport of water or ions through lipid bilayers [50-52].

Due to the complexity of the interactions and the incredibly large number of atoms, atomistic simulations are seriously limited in size. The first simulations 
in the early 1990's included only a hundred lipid molecules that were simulated for $0.1 \mathrm{~ns}[53,54]$, and even today's state of the art simulations on the fastest parallel computers are unable to handle systems larger than a few hundred lipid molecules for much longer than 100 ns [55-57]. Thus, a computer simulation with full atomistic detail of the aforementioned collective phenomena on the interesting time and length scales is inaccessible, at present and in the foreseeable future.

Even if such detailed studies became feasible in the future, they would be plagued by yet another problem which is particularly bothering for a theoretical physicist. The abundance of the degrees of freedom with their highly specific atomistic detail would hide the underlying universal mechanisms and complicate the identification of the relevant degrees of freedom that dominate the phenomenon under study. In other words, "even though one would have all experimental values right, one wouldn't learn anything about the system under study" [58].

Instead of trying to describe the collective phenomena starting from the underlying, atomistic structure, coarse-grained models overcome this difficulty by replacing the microscopic background with a simplified description. This is often achieved by lumping several atoms into effective beads that interact with each other via simplified, effective potentials (see Fig. 1.4). Chemical bonds are typically described by harmonic springs which are sometimes accompanied by bond angle potentials; torsional potentials are normally ignored. The nonbonded interactions are mostly short-ranged, i.e., it is assumed that the longranged Coulomb interactions are screened [59-64].

The determination of these simplified interactions is "often guided by physical intuition, computational constraints, and a large degree of trial-and-error" [65]. Two different approaches for obtaining these interactions can be distinguished, which we will denote as the systematic and the phenomenological coarse-graining. In the former one, a scheme for mapping atoms onto effective beads and a set of basic interactions with many adjustable parameters are proposed. The parameters are fitted in a way that the coarse-grained model reproduces some key quantities as closely as possible, e.g., certain energies or structural quantities. Several of these systematically coarse-grained models for lipid bilayers have been established [59, 66-69].

In the phenomenological or "minimal" models, one relies on the concept of universality, i.e., the assumption that all models, which capture the relevant interactions of a physical system, should show the same qualitative behavior on mesoscopic scales. The strengths of these relevant interactions are set by a few coarse-grained parameters. Unfortunately, in most cases it is unknown what these coarse-grained parameters and the corresponding relevant interactions are. Nevertheless, once they have been found, a model comprising the minimum amount of necessary interactions is obtained which is very appealing from a 

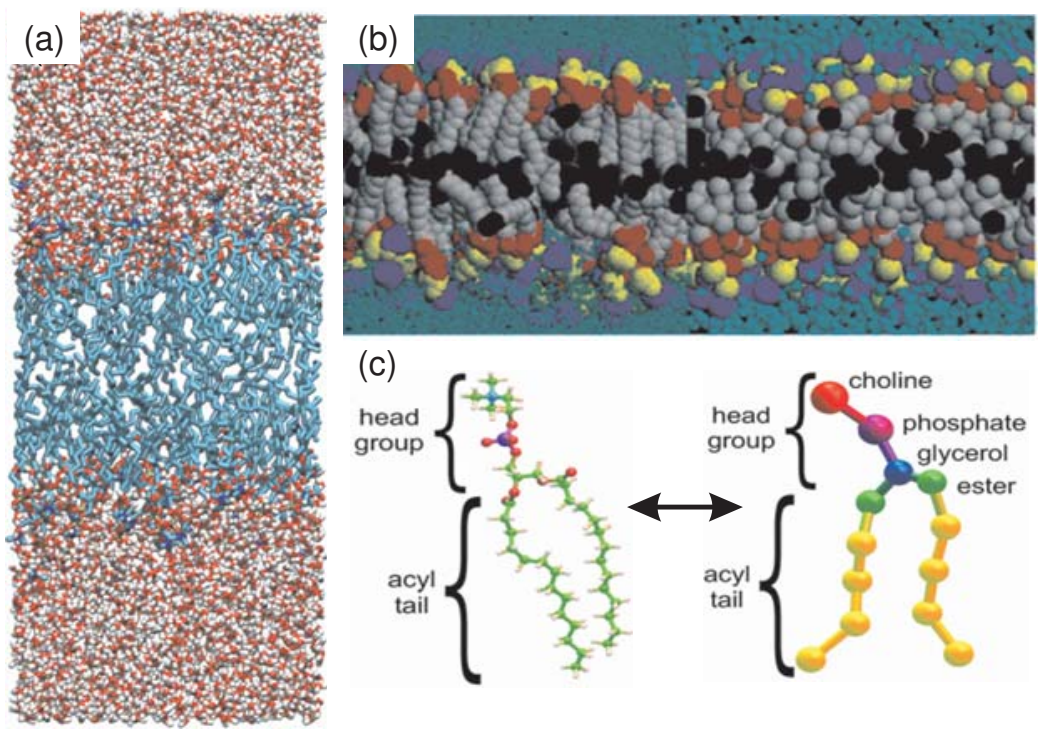

Figure 1.4: (a) Side view of a DPPE bilayer membrane at $T=300 \mathrm{~K}$ surrounded by explicit water molecules (TIP3P). (b) DPPC bilayer in another atomistic model (left) and in a systematically coarse-grained model (right). Reproduced in part with permission from the Journal of Physical Chemistry B [60]. Copyright 2004 American Chemical Society. (c) Atomistic and systematically coarse-grained structure of a DMPC lipid, taken from [61]. Reproduced with permission from IOP Publishing Ltd.

physical point of view and extremely efficient from a technical point of view. Several of these models for lipid bilayers exist [63, 64, 70-76]. Although some of them are excellent in describing lipid bilayers, they all involve interaction coefficients whose physical meaning remains vague.

In both cases, the reduction of the microscopic degrees of freedom has several consequences. First and foremost, significantly less degrees of freedom - compared to atomistic models- have to be simulated for reaching the same length scale. Second, the usage of effective particles leads to softer interactions so that much larger integration time steps than in $\mathrm{MD}(\sim 1 \mathrm{~ns})$ are possible. Third, the coarse-graining is done for a specific thermodynamic state, e.g., pressure, temperature, or thermodynamic phase. Hence, it is understandable that, in general, the effective interactions depend on these parameters [77]. Fourth, the reduction of chemical detail renders the predictions of a coarsegrained model near or below the characteristic scale of the reduced degrees of freedom devoid of any physical meaning. Thus, one has to decide which degrees of freedom shall be studied before one can select the degrees of freedom to be coarse-grained. This decision is the crucial step for the construction of a coarse-grained model. 
Another important aspect in coarse-grained models arises from the treatment of the solvent. Since an amphiphilic bilayer is a two-dimensional object without a bulk phase, most of the volume of the simulation box is occupied by a solvent, whose main purpose is in many cases only to promote the self-assembly. Thus, as long as no hydrodynamic interactions of the solvent are of inherent interest and only the bilayer's properties shall be studied, a simplified treatment or a total omission of the solvent potentially leads to a drastic reduction of the number of interactions to be evaluated and of the required computing time.

The usage of solvent-free models has a long-standing tradition in polymer science. In many polymer models the solvent is taken into account in the interactions between the polymer beads effectively, i.e., slightly attractive interactions mimic a poor solvent and purely repulsive interactions a good solvent. Formally, the removal of a solvent can be justified by treating the system comprised of the polymers and of the solvent as being incompressible on mesoscopic scales with a fixed density $\rho_{0}$. If the solvent's degrees of freedom are integrated out, one obtains a model where the local polymer density, $\rho(\mathbf{r})$, can fluctuate in the interval $0 \ldots \rho_{0}$.

Recently, solvent-free models have been successfully employed in the study of lipid bilayer membranes [64, 66, 70-72, 78-80]. It has turned out that meaningful results of many static properties, like density profiles, bending rigidities, area compressibilities, or the phase behavior, can be obtained in agreement with experiments or atomistic models. These solvent-free models are particularly appealing because only the interesting degrees of freedom of the bilayer are simulated, the desired time and length scales become accessible, and a study of the collective phenomena is qualitatively possible [65, 81, 82].

However, the lack of solvent influences the bilayers. For instance, several solvent-free models exist where the self-assembly into bilayers cannot be demonstrated [70, 78]. From the point of view of statistical physics, the removal of a chemical species leads to a change in the number of thermodynamic degrees of freedom, i.e., in the number of intensive state variables that can be specified independently. Finally, the dissipation of energy, which is dominated by the solvent viscosity on large length scales [83-85], is modified and excitations decay differently.

The effect of coarse-graining on dynamic properties, like dispersion relations, autocorrelation functions, or transport coefficients, is less well understood. The problem is that the microscopic degrees of freedom create friction and thermal noise and act as a heat bath that dominates the time evolution of the mesoscopic degrees of freedom. Coarse-graining always decimates the microscopic degrees of freedom and therefore leads to a reduction of the friction and the thermal noise. This reduction may impact quantities differently, so that the speed-up of the dynamics in a coarse-grained model is desired from a computational point of view, but if there are several slow processes, they may be 
speeded-up by different scaling factors as well. Thus, different relevant slow processes ensue with rates that deviate from the original system. Although much effort is devoted to obtaining an understanding of the coarse-grained dynamics, there is currently no practical scheme for mapping the dynamics [8688]. Hence, as soon as a coarse-grained model is used to study time-dependent phenomena, great care must be exerted to ensure that the dynamics are physically meaningful.

\section{Outline}

In this thesis we rely on the concept of universality. Instead of trying to reproduce chemical details of specific lipid molecules, like it is done in atomistic or systematically coarse-grained simulations, we present a phenomenologically coarse-grained, solvent-free model for amphiphilic bilayers, which smoothly interpolates between lipid and polymer membranes. It is situated somewhere between the systematically coarse-grained and the continuum models, and has some similarities with models used in self-consistent field calculations [89-91]. The interaction coefficients are calculated from simple expressions, which we derive from mean-field theory, and thus offer a direct insight into their physical significance. In Chapter 2 we detail the model description and the simulation method.

However, before studying collective phenomena we have to ensure that the model accurately describes lipid and polymer bilayers. We analyze the statics and dynamics in turn.

In Chapter 3 we begin with the statics and answer the questions which length scales are reachable and which influence the phenomenological coarse-graining has. It turns out that our model exhibits a rich phase diagram with a fluid and several gel phases. Thus, we are able to study lateral phase separation and phase transitions. In the course of these studies we develop a method for precisely locating the point of the fluid-gel transition and measure the interfacial free energy, i.e., the line tension, for a fluid-gel interface.

The first part of Chapter 4 focusses on the question how realistic the coarsegrained dynamics is. To this end we carefully analyze the Brownian motion as well as the collective motion of the amphiphiles, and compare our findings to a recent atomistic molecular dynamics simulation where a flow pattern in a lipid bilayer is observed [92].

We continue the discussion of the dynamics by looking at the consequences which arise from the lack of solvent. The most important modifications show up in the dissipation mechanisms and in the related decay of the undulation modes. Although we discover that the absence of the solvent impacts the dynamics of these modes on large length scales, we obtain an almost quantitative agreement with other simulation studies of the remaining dissipation mecha- 
nisms on smaller scales. Thus, it is possible to use our model for the study of mesoscopic phenomena where dissipation on small scales is important.

In the last part of Chapter 4, we present an even more coarse-grained representation of our model where the molecules are modelled as soft discs which move only in-plane. By treating the dissipation constants as additional coarsegrained parameters, we perform a mapping of our original model that conserves the statics and the dynamics. We present the conditions that have to be fulfilled if the mapping of the dynamics is to be achieved by using a thermostat only.

We close this thesis in Chapter 5 with a summary of the most important findings and an outlook on further developments and extensions of the model. 



\section{Coarse-grained model}

"Everything should be made as simple as possible, but not simpler."

(Albert Einstein)

This chapter is devoted to the description of the soft, coarse-grained model for amphiphilic bilayers used in this work. In Sec. 2.1 we begin with an overview of the different atomistic and coarse-grained models that already exist. We continue in Sec. 2.2 with the physical ideas of our model and present the detailed interaction potentials. Finally, in Sec. 2.3, we describe the method of simulation and focus on the computational implementation.

\subsection{Atomistic and coarse-grained models - an overview}

In general two different types of models are used in the study of bilayer membranes: the "bottom-up" or "ab initio" models and the "top-down" or coarsegrained models. The former ones describe the physical system under study as realistically as possible and computer simulations can be used to predict the results of a variety of experiments accurately [93]. Due to the enormous number of interacting degrees of freedom, these models are limited to the study of very small systems for short time intervals, i.e., only a few hundred amphiphiles embedded in a solvent for tens or hundreds of nanoseconds. The latter ones focus at the interesting time and length scales, microseconds and micrometers, where the collective nature of the interacting molecules becomes important. To this end coarse-grained models integrate out many microscopic degrees of freedom and incorporate the effect of the microstructure into a small number of phenomenological, coarse-grained parameters [65, 81, 82].

The atomistic models, which can be divided into the all-atom models and the united-atom models, are the most important members of the first category. In the all-atom models every single atom of every interacting molecule is included in the simulation, whereas in the united-atom models small groups of atoms, e.g., $-\mathrm{CH}_{3}$ groups, are treated as effective atoms with an effective charge or dipole moment [94, 95]. In both cases much effort is devoted to a realistic description of the interactions between the atoms which requires a careful calibration of all force fields [40-43]. However, since the microscopic interactions lead to harsh repulsive forces, very small time steps, e.g., $\Delta t \approx 10 \mathrm{fs}$, must be 
used in molecular dynamics (MD) simulations and the computational requirements are formidable. Due to the huge number of involved particles, typically $10^{8}-10^{10}$, large amounts of computing time are needed for the simulation of systems whose largest dimension is still comparably small, e.g., < 1 $\mu \mathrm{m}$ (see Fig. 1.4).

The systematically coarse-grained models are closely related. Here, larger groups of atoms, e.g., 5-20, are lumped into effective particles and the interactions between these particles are obtained by a careful matching of static properties, like the radial distribution function [96, 97] or the forces [98-101]. It has been demonstrated [59, 66-69] that these models lead to an accurate description of bilayers on all length scales beyond the size of a single molecule. Due to the coarse-graining, the effective interactions are softer than in the atomistic case and the number of interacting particles is significantly reduced. These models are a very promising approach to reach the relevant time and length scales while preserving realistic interactions even at the small scales.

The other broad category comprises the phenomenologically coarse-grained models. Formally, they can be justified by integrating out the fast, microscopic degrees of freedom below a certain threshold. In this way one obtains effective interactions that depend only on the slow degrees of freedom and a few coarse-grained parameters which incorporate the microstructure. The larger this threshold is, the more microscopic details are integrated out which necessarily leads to deviations in some physical quantities from their experimental counterparts near or below this threshold. Hence, these models cannot be used to study small scales, but they allow a qualitative understanding of the interrelations of the coarse-grained parameters and the physical observables on large scales.

Frequently, the procedure of explicitly integrating out the degrees of freedom is skipped and a Hamiltonian ${ }^{1}$ for a coarse-grained model is proposed directly which depends on a few coarse-grained parameters. These parameters are often matched with values obtained from atomistic simulations or experiments. A common feature of these models is that their interactions are "soft", i.e., the involved energies are comparable to the thermal energy, $k_{B} T$, and therefore smaller than the harsh, microscopic interactions with typical energies of $\mathrm{eV}$. A plethora of different coarse-grained models for amphiphilic bilayers has been proposed which can be roughly divided into different types by their level of coarse-graining. The amount of included microscopic detail roughly follows the evolution of the computational power over the last 30 years.

In continuum models the bilayer is modelled as an smoothly-curved, infinitely thin surface. The molecular structure is assumed to be negligible and the energy depends on macroscopic quantities like curvature or membrane topology.

\footnotetext{
${ }^{1}$ In fact, it is a free energy.
} 
Helfrich's model is certainly the most representative one of this class of models [102-104]. These models can be used to study a large range of cell or vesicle morphologies [105] and to explain mechanical properties on large scales. In simulations these objects are frequently modelled with triangulated surfaces $[106,107]$. However, they ignore the surrounding solvent totally and it is difficult to study non-bilayer-like structures or changes of the topology that occur for instance during membrane fusion or fission [11].

Another type are the molecular models where each molecule is represented by a single particle or an object with a finite amount of states [62, 108-112]. These were mainly used in the past to acquire qualitative insights into the static properties of lipid bilayers, like phase transitions and phase diagrams of various mixtures, i.e., lipid mixtures or mixtures with other molecules like cholesterol. Many of them are lattice models and, consequently, computationally very efficient. However, these simple models cannot be mapped easily to specific kinds of lipids and quantitative results must be taken with a grain of salt. The investigation of dynamical phenomena is outright impossible, because no continuous degrees of freedom exist. Although most of these models have been superseded by flexible off-lattice ones today, they still present a possible route to investigate systems consisting of millions of lipids.

The most important class of modern models suitable for computer simulations are the (off-lattice) bead-spring models where each molecule is represented by a small number of structureless beads connected by springs. Each bead is treated as a point particle with three translational degrees of freedom and interacts with the other beads through pairwise interaction potentials. The beads form flexible molecules which offer several advantages over the molecular models: first and foremost an unlimited number of conformations is accessible, so that even miniscule conformational changes in response to some perturbation can be studied. Furthermore, different molecular architectures, like the number of hydrophobic tails or their length as well as different lipid compositions can be easily implemented.

In contrast to the representation of the amphiphiles, which are in many models similar, there is a broad range of coarse-grained representations for the surrounding solvent; normally this is water. In principle four different ones have been devised so far.

(i) The earliest models include an "explicit solvent" where a small number of water molecules, typically 2-4, are lumped into a single coarse-grained particle in the simulation [63, 74-76, 113]. The interactions between these solvent particles are taken into account explicitly, so that these models normally show realistic hydrodynamics. Additionally, the solvent promotes the self-assembly of the amphiphiles to various morphologies like micelles, bilayers, or vesicles. However, they pay a heavy computational price for this. It is easy to see that a two-dimensional bilayer will occupy only a small amount of space in a three- 
dimensional simulation box and that most of the volume is filled with solvent. Therefore a large amount of the calculated interactions are between "irrelevant" solvent molecules and the whole simulation becomes rather inefficient. Another problem is the artificial structure that the explicit interactions impart to the solvent.

(ii) A first solution to this problem was tried with the introduction of a "surface solvent potential" [114] where no solvent molecules are simulated at all. Instead, the hydrophilic heads couple to an external potential that keeps them at the surface. This potential constrains the bilayer to a plane and strongly damps its undulations. Therefore, the results of this type of model must be interpreted with great care.

(iii) A clever solution for the solvent problem was found by Lenz and Schmid $[73,115]$ who invented a "phantom solvent". In this representation the solvent molecules interact only with the amphiphiles but not with themselves. This has the advantage that the solvent possesses no internal structure, still promotes self-assembly, and is inexpensive to simulate. It can even be used to study the effect of the hydrodynamic coupling between the bilayer and the surrounding fluid.

(iv) As long as the effects of the solvent are of inherent interest to the physical problem, one must naturally include it in the model. Frequently, its only task is to promote hydrophobic attractions between the tails, and as such it is only of minor relevance to the purpose of a simulation. Therefore it is tempting to use "solvent-free" models where the solvent is removed altogether, like it is frequently done in polymer physics, and to take account of it by effective attractions between the hydrophobic tails instead. Such a model is computationally extremely efficient, because only the interesting interactions within the membrane are included. It is known from the earliest attempts of constructing such solvent-free models, that simple Lennard-Jones-like pair potentials lead either to solid bilayers at low temperatures or to dilute gases at higher temperatures. This fact inspired Noguchi and Takasu to add higher order (multi-body), density-dependent interactions and led them to the construction of a first solvent-free model that shows self-assembly [64, 72]. Unfortunately, this model suffers from severe difficulties with the interpretation of thermodynamic quantities. Other models with angular potentials [78] or finely-tuned Lennard-Jones interactions [70] followed. However, the self-assembly cannot be demonstrated for all of these models, and in some cases the bending rigidity of a bilayer is either too high or too low [71]. Since then a small number of solvent-free models have emerged that show self-assembly and have reasonable values for the bending rigidity $[71,79,80]$. These models frequently employ interaction potentials with numbers for the interaction coefficients that "produce the right physics", but cannot be readily understood from a physical point of view. 
In summary, atomistic models have a long-standing tradition in simulations. They provide quantitative results, allow the study of all processes on the atomic level, and are frequently employed to interpret experimental results. However, they are restricted to very small systems and cannot reach the time and length scales where collective effects involving many molecules, microseconds and micrometers, become important. In coarse-grained models many microscopic degrees of freedom are integrated out for these scales to be accessible. This integrating-out is done either systematically or phenomenologically. The first approach yields all-purpose models whose accuracy is on large scales comparable to that of atomistic models. The latter approach yields efficient models, that reproduce key quantities on large scales, but is unable to provide meaningful results on the microscopic scales. Currently, several coarse-grained bead-spring models have been proposed which fundamentally differ in their treatment of the solvent. All of them employ interaction potentials where numerical constants show up whose physical origin remains unclear.

\subsection{Model description I: physical part}

In this work a phenomenologically coarse-grained, solvent-free model for the amphiphilic molecules is considered and this section provides a detailed description from a physical point of view. We discuss the bonded, intramolecular interactions first and then turn to the more involved non-bonded ones.

\subsubsection{Bonded interactions}

In the following the system under study contains $n$ identical molecules and each one consists of $N$ interaction centers which are linearly connected by harmonic springs. There are additional bond angle potentials that stiffen the molecules and restrict their conformational fluctuations. The potential energy is given by

$$
\frac{\mathcal{H}_{\mathrm{b}}}{k_{B} T}=\frac{k_{s}}{2} \sum_{i=1}^{N-1}\left(\left\|\mathbf{r}_{i+1, i}\right\|-\ell_{0}\right)^{2}+k_{b} \sum_{i=2}^{N-1}\left(1-\cos \theta_{i}\right),
$$

where $\mathbf{r}_{i+1, i}=\mathbf{r}_{i+1}-\mathbf{r}_{i}$, and $\mathbf{r}_{i}$ denotes the position of the $i$ th bead of a chain. $k_{s}$ is the spring constant, $\ell_{0}$ defines the position of the minimal potential energy, and $k_{b}$ is the bond angle constant. All simulations take place at the same fixed temperature, $T$, and the thermal energy, $k_{B} T$, serves as the unit of energy in the rest of this work. $\theta_{i}$ denotes the angle between three successive beads, and

$$
\cos \theta_{i}=\hat{\mathbf{r}}_{i+1, i} \cdot \hat{\mathbf{r}}_{i, i-1}
$$

where $\hat{\mathbf{r}}_{i, j}=\mathbf{r}_{i, j} /\left\|\mathbf{r}_{i, j}\right\|$ is a unit vector (see Fig. 2.1). 

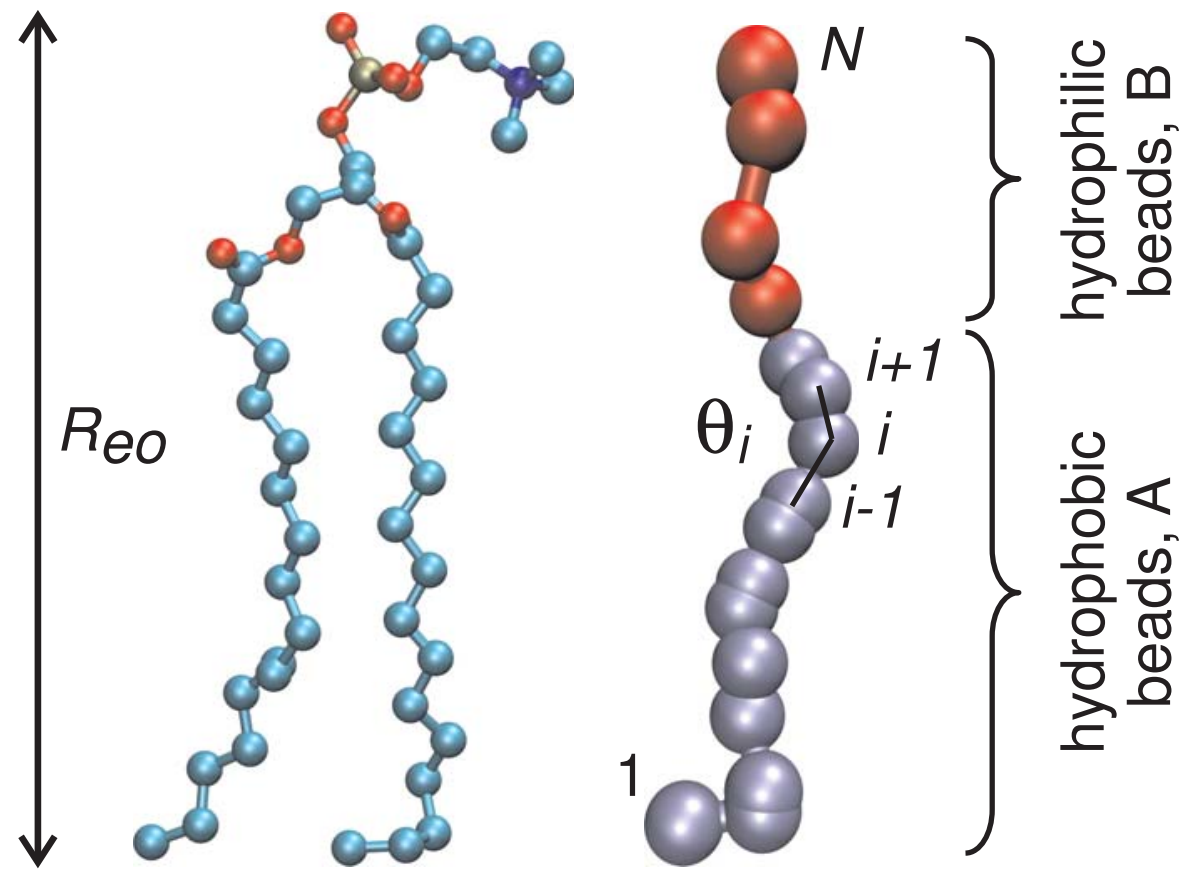

Figure 2.1: Architecture of our coarse-grained lipid molecules (right) in comparison to an united-atom representation of a DPPC molecule (left).

Although amphiphilic molecules are characterized by several length scales, the root-mean-square end-to-end distance $R_{\mathrm{eo}}=\left\langle\left(\mathbf{r}_{N}-\mathbf{r}_{1}\right)^{2}\right\rangle^{1 / 2}$ of molecules that are only subjected to the bonded interactions, is used as the characteristic dimension of a bilayer. $R_{\mathrm{eo}}$ can be pictured as the head-to-tail length of a molecule. The use of $R_{\text {eo }}$ to specify the molecular extension is rooted in polymeric membranes, where the polymer conformations are characterized by this single length scale [116]. The actual size of a molecule is, of course, influenced by the interactions with its neighbors.

One could easily extend the interaction potential in Eq. (2.1) to other molecular architectures, e.g., amphiphiles with two tails. While not being part of the present work, such architectures may be considered in the future.

\subsubsection{Non-bonded interactions}

In this work, each interaction center is either hydrophobic, "A", or hydrophilic, "B". Every molecule consists of two parts, a single hydrophobic tail with $N_{A}$ beads of type A, and a hydrophilic head with $N_{B}=N-N_{A}$ beads of type B (see Fig. 2.1). The non-bonded interactions are incorporated into the model by a phenomenological ansatz for the excess free energy. Specifically, we commence 
with the density functional $[116,117]{ }^{2}$

$$
\frac{\mathcal{H}_{\mathrm{nb}}^{\prime}\left[\hat{\rho}_{A}, \hat{\rho}_{B}\right]}{k_{B} T}=\int \frac{d^{3} r}{R_{\mathrm{eo}}^{3}} \hat{\rho}_{\alpha}(\mathbf{r})\left[\frac{v_{\alpha \beta}}{2} \hat{\rho}_{\beta}(\mathbf{r})+\frac{w_{\alpha \beta \gamma}}{3} \hat{\rho}_{\beta}(\mathbf{r}) \hat{\rho}_{\gamma}(\mathbf{r})\right]
$$

which is a third order expansion in terms of dimensionless, microscopic molecular densities. Here, we use the Einstein sum convention where Greek indices, that occur twice, imply a summation over all species, i.e., $A$ and $B$. The integration extends over the whole simulation box. Due to symmetry there are three expansion coefficients of second order $\left(v_{A A}, v_{A B}, v_{B B}\right)$ and four of third order $\left(w_{A A A}, w_{A A B}, w_{A B B}, w_{B B B}\right)$.

These densities are defined as

$$
\hat{\rho}_{\alpha}(\mathbf{r})=\frac{R_{\mathrm{eo}}^{3}}{N} \sum_{i=1}^{n N} \delta\left(\mathbf{r}_{i}-\mathbf{r}\right) \delta_{\alpha \mathcal{T}(i)}
$$

They count the proportionate number of molecules of species $\alpha$ in a volume $R_{\mathrm{eo}}^{3}$ and are functions of the explicit coordinates, $\mathbf{r}_{i}$, of the beads of species $\alpha$, where $\mathcal{T}(i) \in\{A, B\}$ denotes the species of bead $i$. The prefactor has been chosen such that the molecular density is independent of $N$. Below, we define weighted, molecular densities, where the $\delta$-function in Eq. (2.4) is replaced by a smooth weighting function in order to regularize the terms in Eq. (2.3). Thus, $\mathcal{H}_{\mathrm{nb}}^{\prime}\left[\hat{\rho}_{A}, \hat{\rho}_{B}\right]$ is a function of the explicit particle coordinates and the properties of the coarse-grained model can be studied by computer simulations.

Within the mean-field approximation, the properties of the particle-based simulation model coincide with the results of a classical density functional theory (DFT) calculation using $\mathcal{H}_{\mathrm{nb}}^{\prime}\left[\hat{\rho}_{A}, \hat{\rho}_{B}\right]$. In particular thermodynamic and structural properties decouple [118]: the thermodynamic properties of a spatially homogeneous system, e.g., the EOS, are dictated by the seven expansion coefficients, whereas the local structure is encoded in the definition of the weighted densities.

The advantages of these DFT-based, non-bonded interactions are twofold. On the one hand, Eq. (2.3) can be generalized in a systematic way to accommodate more sophisticated EOSs. In the present work, we use a third-order expansion [89, 90, 119], because this is the simplest form capable of describing all six qualitatively different types of phase diagrams that a compressible binary system exhibits. According to the classification of van Konynenburg and Scott [120, 121], this suffices to capture all qualitative features of the interplay between the liquid-vapor phase separation and the demixing of two

\footnotetext{
${ }^{2}$ This expression should not be confused with a low-density virial expansion around an ideal gas. Equation (2.3) defines our model's equation of state (EOS) and is valid for all densities.
} 
species. Moreover, by virtue of its simplicity, the second and third order coefficients are straightforwardly related to the density and the compressibility of a homogeneous liquid, as well as the incompatibility between hydrophobic and hydrophilic entities. This relation imparts a transparent physical interpretation to the expansion coefficients. $\mathcal{H}_{\text {nb }}^{\prime}\left[\hat{\rho}_{A}, \hat{\rho}_{B}\right]$ also allows for a systematic generalization to systems comprised of more than two different species, a situation which arises in the study of more complex biomembranes.

On the other hand, the weighted densities encode local structural information. Altering the definition of the weighted density, we are able to describe lipid bilayer membranes, which exhibit pronounced packing effects on the length scale of an effective interaction center, or polymersomes that are comprised of long, flexible, amphiphilic polymers and, typically, do not form gel phases.

We discuss how to choose the expansion coefficients and the definition of the weighted densities in turn.

\section{Thermodynamic coefficients}

Formally, we consider the system of amphiphiles and solvent on the mesoscopic scale as an incompressible, dense liquid. Knowing the local densities of amphiphiles, one can reconstruct the solvent density via the incompressibility constraint and integrate out the degrees of freedom associated with the solvent $[65,77]$. This gives rise to effective interactions and the incompressibility constraint generates multi-body interactions. The occurrence of multi-body interactions is natural in the course of coarse-graining and it would also arise during a systematic coarse-graining procedure, where microscopic degrees of freedom are integrated out explicitly.

The coefficients $v_{A A}$ and $w_{A A A}$ dictate the properties of the hydrophobic species in contact with the solvent. In a solvent-free model, the hydrophobic species forms a dense liquid that coexists with a vapor phase, which represents the solvent. Since the solubility of amphiphiles in the solvent is vanishingly small, the osmotic pressure of the vapor phase, which coexists at the temperature $T$ with the liquid, vanishes, $P \approx 0$. Using the mean-field EOS for the pure $A$-component,

$$
\frac{P R_{\mathrm{eo}}^{3}}{k_{B} T} \approx \bar{\rho}_{A}+\frac{v_{A A}}{2}\left(\bar{\rho}_{A}\right)^{2}+\frac{2 w_{A A A}}{3}\left(\bar{\rho}_{A}\right)^{3},
$$

where $\bar{\rho}_{A}$ denotes the average, molecular density of component $A$, we obtain for the average, molecular density, $\bar{\rho}^{*}$, of the liquid with $P=0$

$$
\bar{\rho}^{*} \approx-\frac{3 v_{A A}}{4 w_{A A A}}
$$




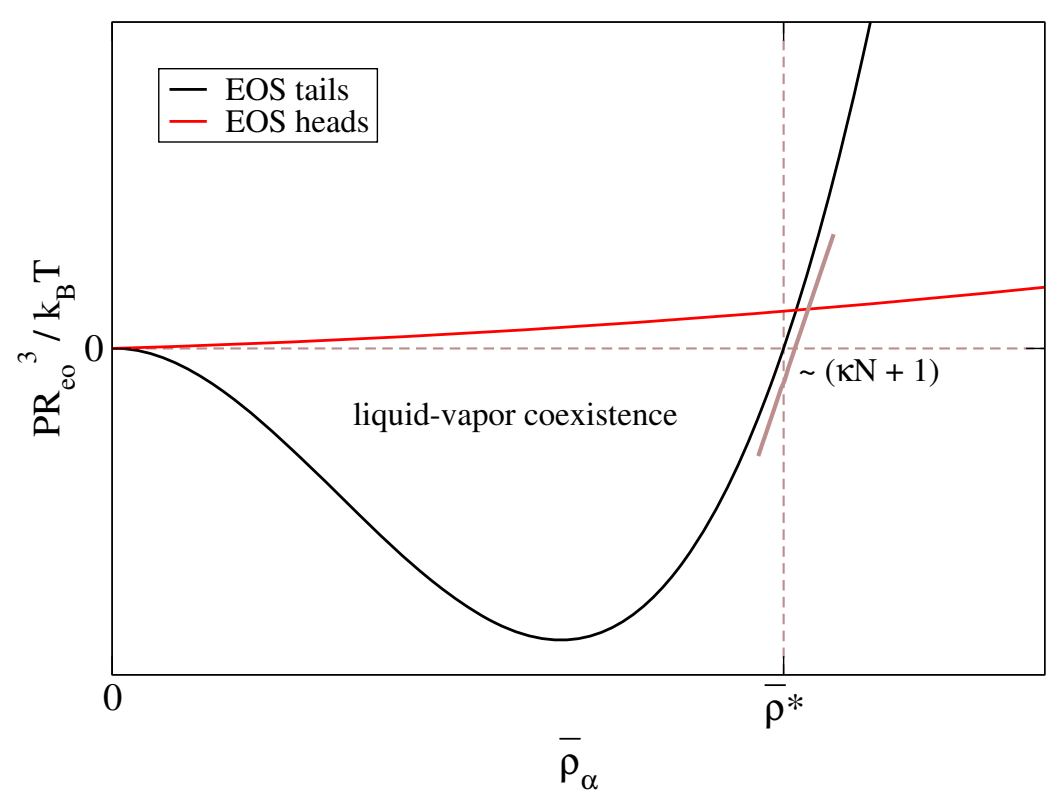

Figure 2.2: Mean-field EOS for the pure $A$-component (black) and the pure $B$ component (red) as a function of the average molecular density, $\bar{\rho}_{\alpha}$. Since the hydrostatic pressure, $P$, is zero, the pure $A$-component phase separates for $\bar{\rho}_{A}<\bar{\rho}^{*}$ into a condensed liquid and an empty vapor phase. $\kappa N$ gives an estimate of the steepness of the EOS at the density of the condensed phase.

and for the dimensionless, inverse compressibility

$$
\kappa N \equiv \frac{R_{\mathrm{eo}}^{3}}{\kappa_{T} \bar{\rho}^{*} k_{B} T}=v_{A A} \bar{\rho}^{*}+2 w_{A A A}\left(\bar{\rho}^{*}\right)^{2}
$$

with $\kappa_{T}=-(1 / V)(\partial V / \partial P)_{T}$, respectively. ${ }^{3}$ These approximate expressions impart a simple physical interpretation to the expansion coefficients (see Fig. 2.2). We will present our results as a function of $\kappa N$ and $\bar{\rho}^{*}$ using the dependencies

$$
v_{A A}=-2 \frac{\kappa N+3}{\bar{\rho}^{*}} \quad \text { and } \quad w_{A A A}=\frac{3}{2} \frac{\kappa N+2}{\left(\bar{\rho}^{*}\right)^{2}} .
$$

At large $\bar{\rho}^{*}$ molecules strongly overlap, packing effects are small, and the system is in the fluid phase. This behavior is typical for polymersomes, where a coarse-grained bead is comprised of many atoms or for high temperatures, where the soft, non-bonded interactions are weak compared to the thermal energy scale. A decrease of $\bar{\rho}^{*}$, in turn, corresponds to an increase of the

\footnotetext{
${ }^{3}$ In both cases we have neglected the contribution of the first term in the EOS, Eq. (2.5), that corresponds to an ideal gas.
} 
repulsive, third-order interactions (cf. Eq. (2.8)), which gives rise to a transition from a disordered to an ordered phase. The coefficient $v_{A B}$ sets the strength of the interactions between $A$ and $B$ beads. It is approximately related to the Flory-Huggins parameter, $\chi N$, via [122]

$$
v_{A B}=\frac{\chi N}{\bar{\rho}^{*}}+\frac{1}{2}\left(v_{A A}+v_{B B}\right) .
$$

$\chi N$ is a dimensionless quantity and measures the incompatibility between hydrophilic and hydrophobic species. The coefficients $v_{B B}$ and $w_{B B B}$ are chosen in a way that the EOS of the hydrophilic beads is purely repulsive, i.e., $v_{B B}=0.1$ and $w_{B B B}=0$. The mixed, third-order coefficients, $w_{A A B}$ and $w_{A B B}$, do not influence the qualitative behavior and, for simplicity, we set $w_{A A A}=w_{A A B}=w_{A B B}{ }^{4}$

Four coarse-grained parameters describe the thermodynamics of our soft, solvent-free, coarse-grained model: $\bar{\rho}^{*}, \kappa N, \chi N$, and $R_{\mathrm{eo}}$. They parameterize (i) the density and (ii) the incompressibility of the hydrophobic interior, (iii) the incompatibility between hydrophilic and hydrophobic beads, and (iv) the spatial extension of an amphiphile. All these parameters are directly related to experimentally accessible quantities and our model can be related to a specific system by matching these four parameters to experimental data.

For instance, we estimate the order of magnitude of $\kappa N$ from the bulk properties of an alkane liquid. Using the isothermal compressibility under standard conditions $\kappa_{T}=0.955 \mathrm{GPa}^{-1}$ for $n$-Dodecane [123], its bulk mass density $\rho_{m}=748.8 \mathrm{~kg} / \mathrm{m}^{3}$, and its molar mass $m=170.34 \mathrm{~g} / \mathrm{mol}$, we obtain $\kappa N=m /\left(\kappa_{T} \rho_{m} k_{B} T\right) \approx 98$. The volume compressibility of lipid bilayers, approximately $\kappa_{T}=0.5 \mathrm{GPa}^{-1}$, is even lower [124, 125] so that the values for $\kappa N$ in Tab. A.1 underestimate the actual values.

It should be pointed out that the thermodynamics of a solvent-free model differs from that of a model including an explicit solvent. The Gibbs phase rule [126],

$$
f=c-M+2,
$$

specifies how many independent thermodynamic variables, $f$, exist in a system with $c$ chemical components and $M$ coexisting phases.

In a single-component bilayer embedded in an explicit solvent there are two components, $c=2$ : the amphiphiles and the solvent molecules. Together they form two phases, $M=2$; a condensed phase with a high amphiphile and a low solvent concentration, and the solvent phase with a very low amphiphile and a high solvent concentration. Hence, $f=2$, i.e., two intensive

\footnotetext{
${ }^{4}$ One could, of course, use some more elaborate mixing rules, like the Lorentz-Berthelot mixing rules [44] or those used by Müller et al. [89].
} 
thermodynamic variables must be specified to define the thermodynamic state. Simulations with an explicit solvent are often performed in thermodynamic ensembles like the $N P_{n} A T, N P_{n} \Sigma T$, or $N P_{t} h T$ one, where $P_{n}$ is the normal (hydrostatic) pressure, $P_{t}$ is the lateral pressure, ${ }^{5} A$ is the area of the bilayer, $\Sigma=h \times\left(P_{n}-P_{t}\right)$ is the mechanical tension, and $h$ is the height of the simulation box in the normal direction [127]. In all ensembles $N$ defines the total number of particles, i.e., the size of the system, but only two of the other three thermodynamic variables are independent. They must be chosen such that the right thermodynamic state is obtained.

In case of a solvent-free model, the amphiphiles, $c=1$, form two phases, the condensed phase and the "vacuum", $M=2$. Hence, $f=1$, i.e., there is only one independent thermodynamic variable. Thus, we simulate our solventfree model in different ensembles, like NVT or $\mathrm{NP}_{t} \mathrm{~T} . V$ and $T$ or $P_{t}$ and $T$, respectively, are in general dependent and must be chosen so that two phases exist. However, $T$ is fixed in our model and the expansion coefficients were chosen such that there is a phase coexistence at exactly this temperature. Hence, $T$ must not be varied, and $V$ or $P_{t}$ are the independent thermodynamic variables that always lead to a condensed phase coexisting with the vacuum. ${ }^{6}$

\section{Local structure and weighting functions}

For lipid bilayer membranes we seek weighted densities that yield a phase diagram with the biologically important fluid phase and, additionally, various gel phases. Analytical studies have suggested that the phase behavior of lipid bilayers is dominated by packing effects due to the excluded volume of the hydrophobic tails [110]. In our model, we can draw on the vast knowledge of liquid-state theory to control the degree of packing effects and the local structure of the fluid in order to tailor the weighted densities such that the fluid exhibits pronounced packing effects.

In order to regularize the $\delta$-function in the excess free-energy functional of non-bonded interactions, Eq. (2.3), we use a weighted-density approximation [128-131] and define coarse-grained densities

$$
\tilde{\rho}_{m \alpha}(\mathbf{r})=\frac{R_{\mathrm{eo}}^{3}}{N} \sum_{i=1}^{n N} w_{m}\left(\left|\mathbf{r}_{i}-\mathbf{r}\right|\right) \delta_{\alpha \mathcal{T}(i)}
$$

by convoluting the microscopic, molecular density, $\hat{\rho}_{\alpha}(\mathbf{r})$, with weighting functions, $w_{m}(r)$ (cf. Eq. (2.4)). These weighting functions are part of the definition of the model and must be chosen with great care. Here, we require that they are

\footnotetext{
${ }^{5} P_{t}$ is the average of the lateral, diagonal components of the pressure tensor.

${ }^{6}$ Unfortunately, this condensed phase is not always a bilayer. Other morphologies, such as micelles or inverted structures are competing.
} 


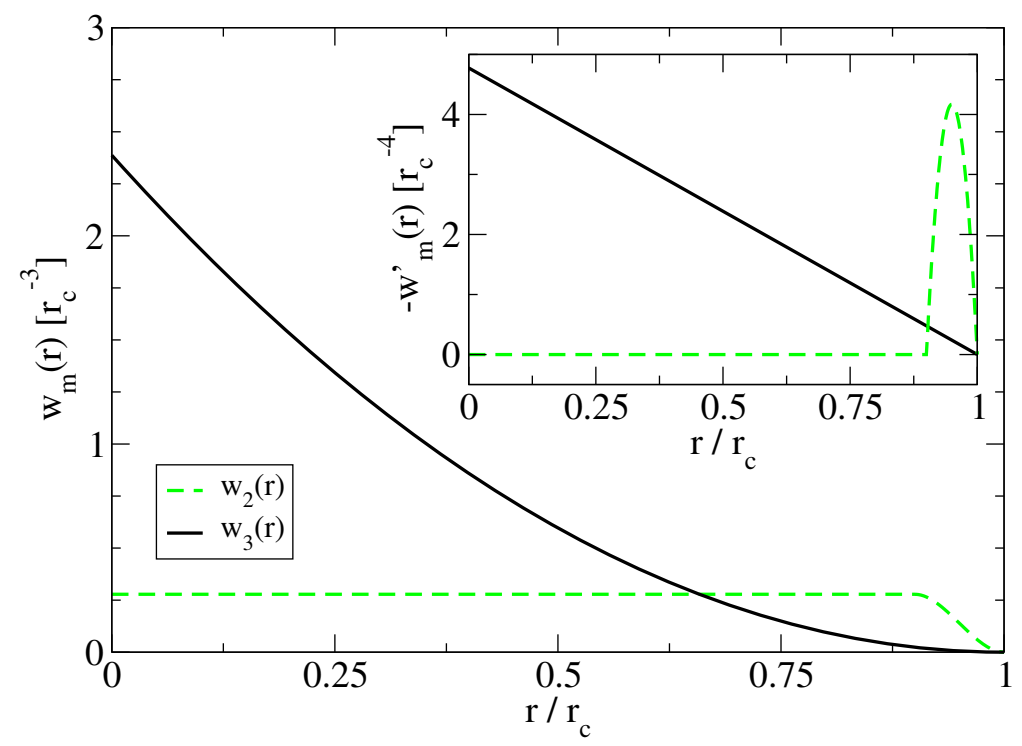

Figure 2.3: The two weighting functions from Eqs. (2.12) and (2.13) with $a=0.9 r_{c}$. The inset shows the negative derivatives of these functions, which are proportional to the non-bonded forces between two beads.

differentiable, vanish for $r \geq r_{c}$, and are normalized, i.e., $\int d^{3} r w_{m}(\|\mathbf{r}\|)=1$. $r_{c}$ defines the cutoff of the non-bonded interactions.

Liquid-state theory for simple liquids [128, 129] as well as integral equation theory $[132,133]$ stress the importance of using different weighting functions to represent the harsh, short-ranged repulsion in a liquid and the soft, longerranged attractions. The second-order terms in Eq. (2.3) typically correspond to attractive interactions and the third-order terms to repulsions. Therefore, we use different weighting functions, $w_{2}(r)$ and $w_{3}(r)$, for the second- and thirdorder contributions. Both weighting functions are plotted in Fig. 2.3. The longer-ranged weighting function,

$$
w_{2}(r)=A \begin{cases}\left(r_{c}-a\right)^{3}, & 0 \leq r<a \\ 2 r^{3}-3\left(a+r_{c}\right) r^{2}+6 a r r_{c}-3 a r_{c}^{2}+r_{c}^{3}, & a \leq r<r_{c} \\ 0, & r \geq r_{c}\end{cases}
$$

consists of a constant part for $r<a$ and a cubic spline for $a \leq r<r_{c}$ with $0<a<r_{c} ; A=-15 / 2 \pi\left(2 a^{6}-3 a^{5} r_{c}+3 a r_{c}^{5}-2 r_{c}^{6}\right)$ is a normalization constant. In this work we use $a=0.9 r_{c}$. The weighting function for the repulsive interactions,

$$
w_{3}(r)=\frac{15}{2 \pi} \times\left\{\begin{array}{ll}
\left(r_{c}-r\right)^{2}, & r \leq r_{c} \\
0, & r \geq r_{c}
\end{array},\right.
$$


is the standard choice in DPD models [134] and only possesses positive Fourier modes. Negative Fourier modes of pairwise, repulsive interactions give rise to cluster-crystallization in dense liquids of soft particles [135-137] and our choice of weighting functions avoids their formation.

Using Eqs. (2.4) and (2.11), we rewrite the non-bonded interactions as

$$
\frac{\mathcal{H}_{\mathrm{nb}}}{k_{B} T}=\int \frac{\mathrm{d}^{3} r}{R_{\mathrm{eo}}^{3}} \hat{\rho}_{\alpha}(\mathbf{r})\left[\frac{v_{\alpha \beta}}{2} \tilde{\rho}_{2 \beta}(\mathbf{r})+\frac{w_{\alpha \beta \gamma}}{3} \tilde{\rho}_{3 \beta}(\mathbf{r}) \tilde{\rho}_{3 \gamma}(\mathbf{r})\right],
$$

which takes the form of a weighted-density functional [128-131]. The densityfunctional form of this coarse-grained interaction free energy controls local correlations, e.g., packing effects. Their length scale is set by the spatial extent of the non-bonded interaction, $r_{c}$. Unlike DFT, however, we obtain the properties not by minimizing the density functional but we use these densityfunctional-inspired interactions in our soft, coarse-grained model whose properties are studied by computer simulation. In this way long-range fluctuations, like undulations, are accounted for.

Finally, we note that weighted densities, which give rise to strong packing effects, deteriorate the quality of the mean-field approximation. Consequently, the decoupling between the thermodynamic properties, like the compressibility and the coexistence density, and the liquid structure breaks down. Therefore, the model parameters, $\bar{\rho}^{*}$ and $\kappa N$, are not identical to the density in the hydrophobic interior of the bilayer and its inverse compressibility. Nevertheless, the approximate equations, (2.6) and (2.7), are a useful guide for constructing the model.

\section{Relationship to Multibody Dissipative Particle Dynamics}

The non-bonded interactions bear a close relationship to another mesoscale simulation method, called "Dissipative Particle Dynamics" (DPD), and in particular to one of its descendants, "Multibody DPD" (MDPD).

Originally, DPD [134, 138-141] was invented for the study of fluid elements pictured as soft, interacting particles. It introduced a momentum conserving, stochastic thermostat that leads to the correct hydrodynamic behavior, so that the DPD fluid adheres to the predictions of the Navier-Stokes equation in the hydrodynamic limit. The great benefit of soft potentials consists in allowing much larger integration time steps than MD to integrate the stochastic equations of motion.

However, the EOS of the original DPD fluid is always quadratic, irrespective of the strength of the interactions [140]. Such an EOS does not permit the simulation of a liquid phase coexisting with its vapor, because this requires at least a third order EOS. This fact stimulated the development of MDPD which 
adds more flexibility with regard to the EOS [142-144]. The method uses as input an EOS in the form of a power series in the density, which would normally produce non-bonded many-body interactions. These many-body interactions are rewritten as density-dependent two-body interactions and an EOS similar to the input is obtained. At this point the weighting functions enter the model definition. Their physical meaning remained unclear in the cited work and, for simplicity, the standard DPD weighting functions were used.

MDPD is, from a mathematical point of view, equivalent to our model, but the physical interpretation differs strongly. First, a soft particle in our case describes a small group of atoms and not a fluid element. Second, the MDPD weighting functions are used to define the local structure of the amphiphiles and liquid-state theory is explicitly used to find meaningful functions. Third, the resulting non-ideal EOS defines our model's EOS; we do not try to tune the model to approximate the mean-field EOS.

\subsection{Model description II: computational part}

MDPD is, from a technical point of view, closely related to MD, but two crucial differences exist: the velocity dependence of the thermostat's forces and the multi-body interactions. Especially the second point is a problem for many standard MD programs [145-148], since most of the publicly available programs neither cope with non-bonded multi-body interactions nor with density-dependent, pairwise interactions. Hence, we have developed our own simulation code. The close relationship to MD enabled us to build on the vast knowledge of molecular simulations and facilitated the usage of optimized parallelization strategies [148-155] or algorithms for efficiently finding interacting particles [156-162].

This section discusses the technical details of our simulation program. First, in Sec. 2.3.1, we describe the force field arising from the interactions and the thermostats. In Sec. 2.3.2 we briefly discuss the two symplectic integration algorithms that were used in this work to simulate either in the canonical (NVT) or the tensionless $\left(\mathrm{NP}_{t} \mathrm{~T}\right)$ ensemble. Finally, we explain our parallelization strategy in Sec. 2.3.3.

\subsubsection{Forcefield}

Like in DPD the force, $\mathbf{F}_{i}$, acting on a bead $i$ consists of three terms,

$$
\mathbf{F}_{i}=\mathbf{F}_{i}^{C}+\mathbf{F}_{i}^{D}+\mathbf{F}_{i}^{R}
$$

The conservative force, $\mathbf{F}_{i}^{C}=\mathbf{F}_{i, \mathrm{~b}}^{C}+\mathbf{F}_{i, \mathrm{nb}}^{C}$, comprises the bonded, $\mathbf{F}_{i, \mathrm{~b}}^{C}$, and the non-bonded forces, $\mathbf{F}_{i, \mathrm{nb}}^{C} ; \mathbf{F}_{i}^{D}$ and $\mathbf{F}_{i}^{R}$, are the dissipative and the random 
force, respectively, coming either from the DPD, the transverse DPD, or the Langevin thermostat. They are discussed in turn.

\section{Conservative forces}

The contributions from the bonded interactions, $\mathbf{F}_{i, \mathrm{~b}}^{C}$, are obtained by taking the derivative of the potential energy in Eq. (2.1) with respect to the coordinates of the beads. Noteworthily, all these forces can be decomposed into pairwise forces which simplifies the calculation of the pressure via the virial theorem. This is obvious for the harmonic springs and can be seen easily for the bond angle potentials by calculating the forces between three beads: ${ }^{7}$

$$
\begin{aligned}
\mathbf{F}_{i-1} & =-\frac{k_{b}}{\left\|\mathbf{r}_{i, i-1}\right\|}\left[\hat{\mathbf{r}}_{i+1, i}-\hat{\mathbf{r}}_{i, i-1} \cos \theta_{i}\right] \\
\mathbf{F}_{i} & =\frac{k_{b}}{\left\|\mathbf{r}_{i, i-1}\right\|}\left[\hat{\mathbf{r}}_{i+1, i}-\hat{\mathbf{r}}_{i, i-1} \cos \theta_{i}\right]-\frac{k_{b}}{\left\|\mathbf{r}_{i+1, i}\right\|}\left[\hat{\mathbf{r}}_{i, i-1}-\hat{\mathbf{r}}_{i+1, i} \cos \theta_{i}\right] \\
\mathbf{F}_{i+1} & =\frac{k_{b}}{\left\|\mathbf{r}_{i+1, i}\right\|}\left[\hat{\mathbf{r}}_{i, i-1}-\hat{\mathbf{r}}_{i+1, i} \cos \theta_{i}\right]
\end{aligned}
$$

Unfortunately, we are unaware of an analytical expression for the single-chain partition function of the potentials in Eq. (2.1), so that the relationship between the parameters $N, R_{\mathrm{eo}}, k_{b}, l_{0}$, and $k_{s}$ must be determined numerically. ${ }^{8}$ To this end a small MC program was used that samples the configuration space of a single, ideal chain with $N$ beads for a given set of interaction parameters, $k_{b}, \ell_{0}$, and $k_{s}$, in an efficient way by using "Slithering Snake" [163, 164] and local displacement moves, until a reliable estimate for $\left\langle\left(\mathbf{r}_{N}-\mathbf{r}_{1}\right)^{2}\right\rangle$ is obtained. If this is smaller than the desired $R_{\mathrm{eo}}^{2}$, then $k_{s}$ is slightly decreased, otherwise $k_{s}$ is increased, and the program runs again with the new set of parameters. Finally, a consistent set of parameters $k_{b}, \ell_{0}$, and $k_{s}$ is obtained, that yields $\left\langle\left(\mathbf{r}_{N}-\mathbf{r}_{1}\right)^{2}\right\rangle \approx R_{\mathrm{eo}}^{2}$. Some important parameter sets for $N=10$ and 16 are listed in Tab. A.2 and Tab. A.3, respectively.

The non-bonded forces, $\mathbf{F}_{i, \mathrm{nb}}^{C}$, stem from the density-dependent Hamiltonian $\mathcal{H}_{\mathrm{nb}}$, cf. Eq. (2.14). We rewrite Eq. (2.14) in a computationally convenient form using Eq. (2.4) and arrive at

$$
\frac{\mathcal{H}_{\mathrm{nb}}}{k_{B} T}=\sum_{i} \delta_{\alpha \mathcal{T}(i)}\left[\frac{v_{\alpha \beta}}{2 N} \tilde{\rho}_{2 \beta}\left(\mathbf{r}_{i}\right)+\frac{w_{\alpha \beta \gamma}}{3 N} \tilde{\rho}_{3 \beta}\left(\mathbf{r}_{i}\right) \tilde{\rho}_{3 \gamma}\left(\mathbf{r}_{i}\right)\right] .
$$

By taking the negative derivative and using Eq. (2.11) we obtain

\footnotetext{
${ }^{7}$ It is a good idea to limit these forces to a maximum value, because otherwise numerical instabilities may occur, when $\left\|\mathbf{r}_{i+1, i}\right\|$ or $\left\|\mathbf{r}_{i, i-1}\right\|$ become small.

${ }^{8}$ There is an expression for $k_{b}=0$ and $\ell_{0}=0: k_{s}=3 k_{B} T(N-1) / R_{\mathrm{eo}}^{2}$.
} 


$$
\begin{aligned}
\mathbf{F}_{i, \mathrm{nb}}^{C} & =-\frac{\partial}{\partial \mathbf{r}_{i}} \mathcal{H}_{\mathrm{nb}}=\frac{k_{B} T R_{\mathrm{eo}}^{3}}{N^{2}} \sum_{j} \hat{\mathbf{r}}_{i j}\left[v_{\mathcal{T}(i) \mathcal{T}(j)} w_{2}^{\prime}\left(\left|\mathbf{r}_{j}-\mathbf{r}_{i}\right|\right)\right. \\
& \left.+\frac{2 w_{\mathcal{T}(i) \mathcal{T}(j) \alpha} R_{\mathrm{eo}}^{3}}{3 N} w_{3}^{\prime}\left(\left|\mathbf{r}_{j}-\mathbf{r}_{i}\right|\right)\left(\tilde{\rho}_{3 \alpha}\left(\mathbf{r}_{i}\right)+\tilde{\rho}_{3 \alpha}\left(\mathbf{r}_{j}\right)\right)\right]
\end{aligned}
$$

Thus, the total non-bonded force is decomposed into a sum of densitydependent pairwise forces, $\mathbf{F}_{i, \mathrm{nb}}^{C}=\sum_{j} \mathbf{F}_{i j}$. The actual evaluation of the force requires an additional calculation of all weighted densities, $\tilde{\rho}_{3 \alpha}\left(\mathbf{r}_{i}\right)$, first. However, this additional calculation is cheap, because it involves only an additional loop over the list of interacting particles.

\section{Standard DPD thermostat}

We use the standard DPD thermostat $[138,139,141,165]$ for most of the simulations. It is Galilean invariant, conserves momentum and angular momentum, and acts locally. The dissipative force, $\mathbf{F}_{i}^{D}\left(\mathbf{r}_{i j}, \mathbf{v}_{i j}\right)$, depends on the distance, $\mathbf{r}_{i j}=\mathbf{r}_{i}-\mathbf{r}_{j}$, as well as on the difference of the velocities, $\mathbf{v}_{i j}=\mathbf{v}_{i}-\mathbf{v}_{j}$, between two interacting particles. They have the same cutoff as the non-bonded interactions, i.e., $r_{c}$, and vanish for $r_{i j}=\left\|\mathbf{r}_{i j}\right\| \geq r_{c}$. For $r_{i j}<r_{c}$ they are given by the standard DPD form,

$$
\begin{aligned}
\mathbf{F}^{D}\left(\mathbf{r}_{i j}, \mathbf{v}_{i j}\right) & =-\gamma w^{D}\left(r_{i j}\right)\left(\mathbf{v}_{i j} \cdot \hat{\mathbf{r}}_{i j}\right) \hat{\mathbf{r}}_{i j} \\
\mathbf{F}^{R}\left(\mathbf{r}_{i j}\right) & =\xi w^{R}\left(r_{i j}\right) \theta_{i j} \hat{\mathbf{r}}_{i j} .
\end{aligned}
$$

The friction constant, $\gamma$, is related to the noise coefficient, $\xi$, by the fluctuation dissipation theorem, $\xi^{2}=2 k_{B} T \gamma . \theta_{i j}$ is a stochastic variable with $\left\langle\theta_{i j}\right\rangle=0$ and $\left\langle\theta_{i j}(t) \theta_{k l}\left(t^{\prime}\right)\right\rangle=\left(\delta_{i k} \delta_{j l}+\delta_{i l} \delta_{j k}\right) \delta\left(t-t^{\prime}\right)$. The random numbers are drawn from a uniform distribution [166] and the standard weighting functions for DPD [134],

$$
\left[w^{R}(r)\right]^{2}=w^{D}(r)= \begin{cases}\left(1-r / r_{c}\right)^{2}, & r<r_{c} \\ 0, & r \geq r_{c}\end{cases}
$$

are employed.

Unfortunately, there are two problems with this thermostat. First, the stochastic nature of the random force can cause problems with the microscopic reversibility. Second, the dissipative force of a particle depends on the velocities of the other particles. At first sight this looks like a minor detail, but it invalidates the widely used integration schemes, for instance the "Velocity Verlet" algorithm. This causes deviations of the actual temperature from the desired one. Several improved or "self-consistent" integration schemes have been proposed over the years which alleviate these problems [167-170], however, it is still an open question how to integrate the stochastic equations of 
motion best. It seems that a different thermostat could be able to solve these problems [171].

In this work we ignore these two problems and accept that our temperature is not exactly unity, but

$$
\frac{\left\langle\mathbf{v}_{i}^{2}\right\rangle}{3 k_{B} T}=1.0028(2)
$$

\section{Transverse DPD thermostat}

The transverse DPD thermostat (TDPD) works exactly the same as the DPD thermostat, there are just additional forces acting perpendicular to the distance vector $\mathbf{r}_{i j}$ [172]; the functional form of these transverse forces is the same as that of the longitudinal ones. To avoid confusion, we now distinguish between the longitudinal friction and noise coefficients, $\gamma^{\|}$and $\xi^{\|}$, and their transverse counterparts, $\gamma^{\perp}$ and $\xi^{\perp}$. The forces between two interacting particles are

$$
\begin{aligned}
\mathbf{F}^{D}\left(\mathbf{r}_{i j}, \mathbf{v}_{i j}\right) & =-w^{D}\left(r_{i j}\right)\left[\left(\gamma^{\|}-\gamma^{\perp}\right) \hat{\mathbf{r}}_{i j}\left(\hat{\mathbf{r}}_{i j} \cdot \mathbf{v}_{i j}\right)+\gamma^{\perp} \mathbf{v}_{i j}\right], \\
\mathbf{F}^{R}\left(\mathbf{r}_{i j}\right) & =w^{R}\left(r_{i j}\right)\left[\left(\xi^{\|}-\xi^{\perp}\right) \hat{\mathbf{r}}_{i j}\left(\hat{\mathbf{r}}_{i j} \cdot \boldsymbol{\theta}_{i j}\right)+\xi^{\perp} \boldsymbol{\theta}_{i j}\right] .
\end{aligned}
$$

The longitudinal and transverse friction and dissipation constants obey the same fluctuation dissipation theorem as before. $\boldsymbol{\theta}_{i j}$ is now a random vector with $\left\langle\boldsymbol{\theta}_{i j}\right\rangle(t)=0$ and $\left\langle\boldsymbol{\theta}_{i j}(t) \cdot \boldsymbol{\theta}_{k l}\left(t^{\prime}\right)\right\rangle=\left(\delta_{i k} \delta_{j l}+\delta_{i l} \delta_{j k}\right) \delta\left(t-t^{\prime}\right)$. $\gamma^{\|}$and $\gamma^{\perp}$ can be chosen independently.

\section{Langevin thermostat}

The Langevin thermostat is the oldest of the discussed thermostats and has been used for long times in "Brownian Dynamics" simulations [44]. It violates the conservation of momentum, thus, it is not Galilean invariant. The stochastic forces acting on particle $i$ are given by:

$$
\mathbf{F}_{i}^{D}=-\gamma \mathbf{v}_{i}, \quad \mathbf{F}_{i}^{R}=\xi \boldsymbol{\theta}_{i}
$$

As before $\gamma$ is the friction constant and related to the noise coefficient, $\xi$, by $\xi^{2}=2 k_{B} T \gamma$. The random vector, $\boldsymbol{\theta}_{i}(t)$, obeys $\left\langle\boldsymbol{\theta}_{i}(t)\right\rangle=0$ and $\left\langle\boldsymbol{\theta}_{i}(t)\right.$. $\left.\boldsymbol{\theta}_{j}\left(t^{\prime}\right)\right\rangle=\delta_{i j} \delta\left(t-t^{\prime}\right)$. As before, we draw the random numbers from a uniform distribution.

\subsubsection{Simulation in various ensembles}

The bilayers were simulated either in the canonical ensemble (NVT), where the dimensions of the simulation box are fixed, or in an ensemble with vanishing 
lateral pressure $\left(\mathrm{NP}_{t} \mathrm{~T}\right)$, where the two lateral box dimensions could fluctuate either isotropically or anisotropically. In MD and DPD simulations the thermodynamic ensemble is closely related to the integration scheme for the equations of motion and a general strategy for deriving arbitrary, phase-space conserving, "symplectic", integration schemes exists [173, 174]. Here, we briefly present the two integration algorithms that were used in this work and that correspond to the two ensembles.

\section{Canonical ensemble (NVT)}

The standard way of integrating the equations of motion in the canonical ensemble is the "Velocity Verlet" algorithm [173]. The momentum, $\mathbf{p}(t)$, and the coordinate, $\mathbf{r}(t)$, of every particle are updated according to the following scheme:

$$
\begin{aligned}
\mathbf{p}(\Delta t / 2) & =\mathbf{p}(0)+\frac{\Delta t}{2} \mathbf{F}(\mathbf{r}(0)) \\
\mathbf{r}(\Delta t) & =\mathbf{r}(0)+\frac{\Delta t}{m} \mathbf{p}(\Delta t / 2) \\
\mathbf{p}(\Delta t) & =\mathbf{p}(\Delta t / 2)+\frac{\Delta t}{2} \mathbf{F}(\mathbf{r}(\Delta t))
\end{aligned}
$$

After $\mathbf{r}(\Delta t)$ has been calculated, all forces, $\mathbf{F}(\mathbf{r}(\Delta t))$, evaluated from the coordinates at time $\Delta t$, must be recalculated. $\Delta t$ is the integration time step and we used $\Delta t=0.005 \tau$ in all simulations. We define the mass of a single bead as unity, i.e., $m \equiv 1$. In combination with the units of length, $r_{c}$, and energy, $k_{B} T$, we obtain the unit of time, $\tau \equiv \sqrt{r_{c}^{2} / k_{B} T}$, that is mapped to an experimental time scale later on.

\section{Tensionless ensemble (NP $\left.\mathbf{P}_{t} \mathbf{T}\right)$}

The integration scheme in the tensionless ensemble is slightly more involved, because the permanently changing box dimensions lead to a rescaling of the lateral components of the particle coordinate vectors. Similar algorithms have already been published for this ensemble [127, 175, 176]. Here, we briefly present our integration algorithm whose derivation follows that of Kolb and Dünweg for an NPT ensemble [177]. The full derivation is given elsewhere [178].

Our coordinate system is chosen such that $x$ is the normal direction, and $y$ and $z$ are the lateral directions. Due to the anisotropy, the lateral and the normal components are treated differently. An additional degree of freedom, $A(t)$, which is the area of the isotropically fluctuating bilayer, with its canonically conjugated momentum, $\pi_{A}(t)$, and a mass $Q$ is introduced. The lateral box 
dimensions are $L_{y}(t)=L_{z}(t)=\sqrt{A(t)} . L_{x}$ is the fixed height of the simulation box and physically meaningless. The current lateral pressure, $P_{t}$, is defined over the diagonal components of the pressure tensor, $P_{\alpha \alpha}$, by $P_{t}=\left(P_{y y}+P_{z z}\right) / 2$. Using the same notation as in the canonical ensemble, the first steps are:

$$
\begin{aligned}
p_{x}(\Delta t / 2) & =p_{x}(0)+\frac{\Delta t}{2} F_{x}(\mathbf{r}(0)) \\
p_{y / z}^{\prime} & =p_{y / z}(0)+\frac{\Delta t}{2} F_{y / z}(\mathbf{r}(0)) \\
\pi_{A}(\Delta t / 2) & =\pi_{A}(0)+\frac{\Delta t}{2} L_{x} P_{t} \\
A(\Delta t / 2) & =A(0)+\frac{\Delta t}{2 Q L_{x}^{2}} \pi_{A}(\Delta t / 2) \\
r_{x}(\Delta t) & =r_{x}(0)+\frac{\Delta t}{m} p_{x}(\Delta t / 2) \\
r_{y / z}^{\prime} & =r_{y / z}(0)+\frac{A(0)}{A(\Delta t / 2)} \frac{\Delta t}{m} p_{y / z}^{\prime} \\
A(\Delta t) & =A(\Delta t / 2)+\frac{\Delta t}{2 Q L_{x}^{2}} \pi_{A}(\Delta t / 2)
\end{aligned}
$$

After this step the particle coordinates must be rescaled using $A(\Delta t)$ :

$$
r_{y / z}(\Delta t)=\frac{\sqrt{A(\Delta t)}}{\sqrt{A(0)}} r_{y / z}^{\prime} \quad \quad p_{y / z}^{\prime \prime}=\frac{\sqrt{A(0)}}{\sqrt{A(\Delta t)}} p_{y / z}^{\prime}
$$

Finally, the forces must be recalculated. For simplicity we use the coordinates $\mathbf{r}(\Delta t)$ and the auxiliary momenta $p_{x}(\Delta t / 2)$ and $p_{y / z}^{\prime \prime}$. The final steps of the integration algorithm are:

$$
\begin{aligned}
\pi_{A}(\Delta t) & =\pi_{A}(\Delta t / 2)+\frac{\Delta t}{2} L_{x} P_{t} \\
p_{x}(\Delta t) & =p_{x}(\Delta t / 2)+\frac{\Delta t}{2} F_{x}(\mathbf{r}(\Delta t)) \\
p_{y / z}(\Delta t) & =p_{y / z}^{\prime \prime}+\frac{\Delta t}{2} F_{y / z}(\mathbf{r}(\Delta t))
\end{aligned}
$$

To damp possible oscillations of $A(t)$, we couple a "Langevin piston" to $A(t)$ by the replacement [177]

$$
\frac{\Delta t}{2} L_{x} P_{t} \rightarrow \frac{\Delta t}{2} L_{x} P_{t}-\gamma_{A} \frac{\Delta t}{2 Q L_{x}^{2}} \pi_{A}+\sqrt{k_{B} T \gamma_{A} \Delta t / 2} \xi_{A} .
$$

$\gamma_{A}$ is the friction coefficient of the area and $\xi_{A}$ is a Gaussian random number with unit variance. We use $Q=0.0001$ and $\gamma_{A}=0.1$. 
A second integration algorithm with two additional degrees of freedom $\left(L_{y}\right.$, $\left.L_{z}\right)$ can be derived in a similar way. It is used to simulate bilayers in the gel phase, where isotropic fluctuations of the area are inappropriate due to the hexatic coordination of the molecules. For brevity the details of this algorithm are omitted.

\subsubsection{Parallelization strategy}

In this subsection we describe the technical implementation of our parallel simulation program. Due to the large spatial heterogeneity caused by the lack of solvent, we decided to employ the "force-decomposition" (FD) strategy [148152] for parallelizing the simulation program. Unlike the domain decomposition scheme, in which distinct parts of the simulation box are distributed over the available compute nodes, the basic idea of the FD algorithm is to subdivide the quadratic matrix of all possible pairwise interactions into smaller submatrices and to assign each one to a different compute node. Specifically, we employ a modified version of Plimpton's FD algorithm [148, 149] that is capable of running on any number of processors and exploits Newton's third law, so that every interaction is computed only once. This facilitates a straightforward incorporation of the DPD or the TDPD thermostat. It is a general scheme for parallelizing any problem where frequent, pairwise computations between many objects are needed. ${ }^{9}$

\section{Force-decomposition parallelization}

Let $P \in \mathbb{N}$ denote the number of parallel processes. The processes are arranged in a two-dimensional Cartesian grid process topology with $R$ rows and $C$ columns. $R, C \in \mathbb{N}$ are arbitrary integers, but must be chosen such that the relation $P=C \times R$ holds. As shown in Fig. 2.4 the processes are ordered in a way that the process with rank $n_{r}, n_{r}=0 \ldots P-1$, is located at the grid point $\left(n_{1}, n_{2}\right), n_{1}=0 \ldots R-1, n_{2}=0 \ldots C-1$ with $n_{1}=n_{r} \div C$ and $n_{2}=n_{r}$ $\bmod C$. $^{10}$

To simplify the discussion we now introduce the expression of a "group" of molecules. Here, a group, $\mathcal{G}$, is an ordered set of molecular indices. If the molecule with index $i \in \mathcal{G}$ has $N_{i}$ beads, then we define the size of a group $\mathcal{G}$, $|\mathcal{G}|$, as the sum

$$
|\mathcal{G}|=\sum_{i \in \mathcal{G}} N_{i} .
$$

\footnotetext{
${ }^{9}$ For instance, it can also be used in the calculation of pair correlation functions.

${ }^{10}$ The integer division, $n_{1} \div n_{2}$, yields the result of the division $n_{1} / n_{2}$ with the fractional part being truncated. The modulus operation, $n_{1} \bmod n_{2}$, yields the remainder of the integer division $n_{1} \div n_{2}$.
} 

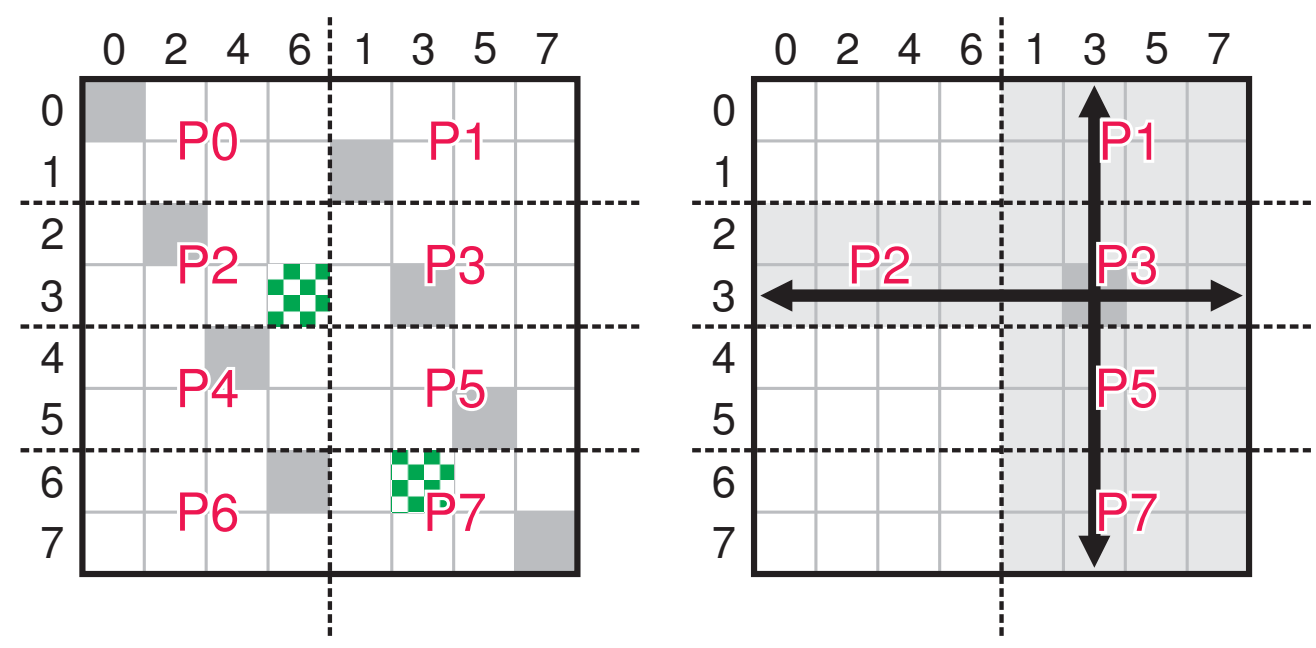

Figure 2.4: Left: Interaction matrix for $P=8, R=4$, and $C=2$ columns. The numbers on the vertical and the horizontal axis are the indices $i$ of the groups $\mathcal{G}_{i}$. Each process has to compute the pairwise interactions $i \leftrightarrow j$ where the bead $i$ is in one of its two groups on the vertical axis and bead $j$ is in one of its four groups on the horizontal axis. The dashed lines denote the borders of the areas of responsibility for each process. The dark gray fields denote the intersections of identical row and column groups. To avoid the double calculation of all interactions, for instance in the green checkered areas, we employ Plimpton's checkerboarding method. Right: Schematic representation of the communication pattern for process 3 as it is used in the computation of the non-bonded forces. Only the gray shaded processes compute forces involving $\mathcal{G}_{3}$, and only those parts of the interaction matrix covered by the thick black arrow need to be communicated to process 3 .

The size is simply the number of all beads belonging to all molecules in a group. A group can also be conceived as a collection of bead indices which are compiled in a way that all beads of one molecule are always contained in the same group.

We now define $P$ groups, $\mathcal{G}_{i}, i=0 \ldots P-1$, one for each process. $\mathcal{G}_{i}$ is called the "local group" of the process with rank $i$. The $n$ molecules of the physical system are distributed among these groups, in a way that (i) every molecule is contained in exactly one group and (ii) all groups have roughly the same size. It is advantageous if all groups acquire exactly the same size. This can be achieved by adding an extra "ghost" molecule, $j$, to group $\mathcal{G}_{j}$ with $N_{j}$ chosen so large that

$$
\left|\mathcal{G}_{0}\right|=\left|\mathcal{G}_{1}\right|=\cdots=\left|\mathcal{G}_{j}\right|=\cdots=\left|\mathcal{G}_{P-1}\right| \text {. }
$$

In principle every bead of every molecule can have non-bonded pairwise interactions with every other bead, or, to use the group terminology, every bead in every group can interact with every bead in every other group. This leads to 
Plimpton's picture of the interaction matrix as it is depicted in Fig. 2.4. Every pairwise interaction occurs twice in this matrix, and we strictly distinguish between the situation where a bead $i$ interacts with a bead $j$, denoted as $i \leftrightarrow j$, and the -physically equivalent- situation where $j$ interacts with $i, j \leftrightarrow i$. If we talk about all interactions of the beads in one group, $\mathcal{G}_{i}$, with the beads in another group, $\mathcal{G}_{j}$, then this is denoted as $\mathcal{G}_{i} \leftrightarrow \mathcal{G}_{j}$.

The central idea of the FD algorithm is to distribute the entire area of the interaction matrix among all processes. In contrast to Plimpton's original work, we do not use the bead indices, but the group indices to label the two axes. In a pairwise interaction, $\mathcal{G}_{i} \leftrightarrow \mathcal{G}_{j}$, the first group -in this case $\mathcal{G}_{i}$ - always corresponds to a group on the vertical axis, while the second group $-\mathcal{G}_{j}-$ always refers to a group on the horizontal axis. The same convention is also used for bead indices.

The groups on the vertical axis are written down in order. Since $P$ is divisible by $R$ without remainder, the processes in row $i$ calculate the interactions of the groups $\mathcal{G}_{(i+k) \times C}$, where $k=0 \ldots C-1$. The groups on the horizontal axis are written down in a permutated order, which goes back to Plimpton. Here, the processes in column $j$ calculate the interactions of the groups $\mathcal{G}_{j+k \times C}$, where $k=0 \ldots R-1$. The reason for this permutation lies in the fact that all processes which compute interactions with group $\mathcal{G}_{k}$ are located within the same row or the same column.

\section{Communications}

The beads' forces are computed in parallel according to the following algorithm. In the initial state every process is aware of the current positions and velocities of all particles in the groups of its row and its column. In a first step, a loop over all interacting pairs of beads within the available groups is performed to calculate the partial weighted densities according to Eq. (2.11). No single process has full knowledge of all interacting pairs, so that a first communication step is required to add the partial weighted densities and to obtain the complete weighted densities, $\tilde{\rho}_{m \alpha}\left(\mathbf{r}_{i}\right)$. The permutation of the group indices has led to the situation in which all processes, that compute interactions of the group $\mathcal{G}_{k}$, are located within a single row and a single column. The process at the grid point where this row and this column intersect is the one with process rank $k$, i.e., $\mathcal{G}_{k}$ is its local group. Hence, it is sufficient to perform two collective communication steps. First, the partial weighted densities of the groups on the vertical axis are summed up among the processes on the same row. Second, the partial weighted densities of the groups on the horizontal axis are summed up among the processes of the same column. Every process participates in two communications and has full knowledge of the weighted densities of its local group at the end. By using two more collective communication steps the 
weighted densities of its local group are then sent back to the other processes on the same row and the same column.

Now, every process has full knowledge of the positions, velocities, and weighted densities of all beads in all its groups. Hence, the non-bonded, conservative forces, Eq. (2.17), and the stochastic DPD forces, Eqs. (2.18-2.19), can be evaluated. For obtaining the full non-bonded forces we need the same communication steps as before, i.e., two summations along the row and the column. After that, every process has full knowledge of the non-bonded forces acting on the beads of its local group.

The calculation of the bonded, intramolecular interactions for the local group is trivial, because all bead coordinates are available. If these bonded forces are added to the previously communicated non-bonded ones, then every process yields the total forces acting on the beads of its local group. With this information, the equations of motion of the local group's beads are integrated by one time step. Finally, the new coordinates and velocities are communicated to the processes in the same row and column, they get back to the initial state, and the procedure starts again.

The method presented so far is already able to compute the non-bonded interactions in parallel. But up to now, every interaction would be computed twice, i.e., $i \leftrightarrow j$ and $j \leftrightarrow i$. According to Newton's third law both conservative forces differ only by a sign, so that one computation is redundant. Plimpton proposed a simple method which he calls "checkerboarding of the interaction matrix" to get rid of this redundancy [148]. His idea is to compute an interaction $i \leftrightarrow j$, only if (i) $i>j$ and $i+j$ is even, or (ii) $i<j$ and $i+j$ is odd. Graphically, this leads to a perforated checkerboard of the interaction matrix: the red squares above the diagonal and the black squares below the diagonal are omitted. This is pictured for the interactions $\mathcal{G}_{3} \leftrightarrow \mathcal{G}_{6}$ and vice versa in the left part of Fig. 2.4. It should be pointed out that Plimpton devised his checkerboarding on the level of bead indices, but it can also be done on the level of molecular or even group indices. Here we use the checkerboarding method on the level of bead indices.

The parallel scalability of our program is shown in Fig. 2.5.

\section{Construction of neighbor list}

Even though we have distributed the force matrix over many processes, we still need an efficient way to find interacting beads within the set of the possible interactions in every process, i.e., where $r \leq r_{c}$ and $r$ is the distance between two beads. To our understanding the most efficient way to find these pairs is to create neighbor lists from cell lists [46].

Many different algorithms for creating cell lists have been proposed over the years [156-162] which can be divided into two categories. Either they subdivide 


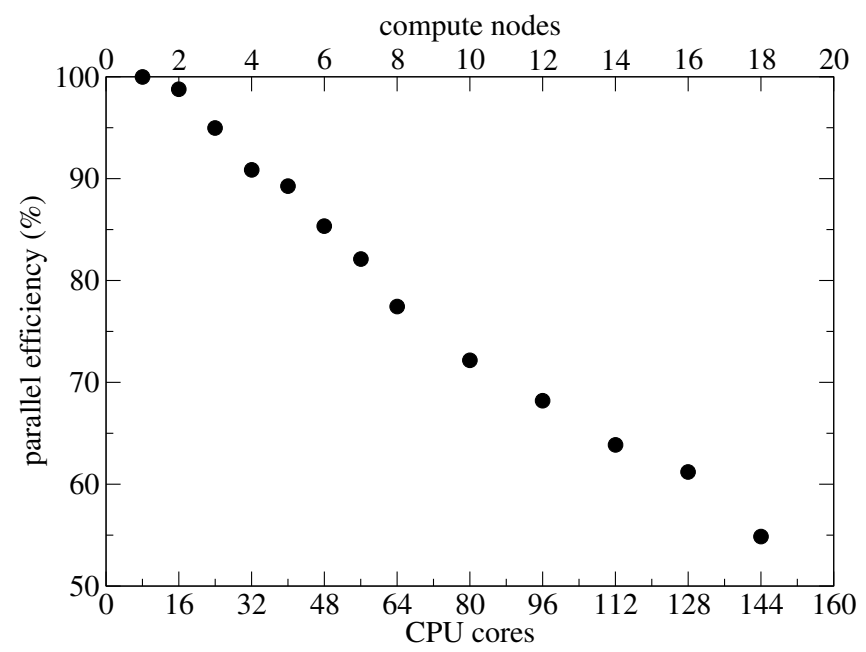

Figure 2.5: Parallel scalability of our simulation code

the simulation volume into many small cells whose size is much smaller than $r_{c}$, or they subdivide it into cells with a size larger than or equal to $r_{c}$. In the former case there are typically zero or one beads in a cell, whereas in the latter case there are significantly more.

We decided to use the fast algorithm for creating the cell lists devised by Heinz and Hünenberger [159] which utilizes small cells. Although this algorithm has been developed for domain-decomposition parallelization schemes, it can be easily adapted to the case of FD. In the NVT ensemble it is used to determine all potential interactions within $r_{c}+r_{s}$, where $r_{s}$ is the shell size of the neighbor list. Here we use $r_{s} / r_{c}=0.07$. In the $\mathrm{NP}_{t} \mathrm{~T}$ ensemble we simply use it to determine the interactions within $r_{c}$. 


\section{Static properties}

"Facts are the air of scientists.

Without them you can never fly."

(Linus Pauling)

The equilibrium properties of lipidic and polymeric bilayers have been studied intensively over the last decades by a multitude of experiments, computer simulations, and analytical calculations. Thus, we commence the discussion of our coarse-grained model with a comparison to these well-known properties. This discussion serves a twofold purpose: first, we determine meaningful values for all parameters, and second we list our results as a reference for future work. The discussion is partitioned into three sections.

In Sec. 3.1 we look at the self-assembled morphologies and determine under which conditions stable bilayers form. We setup the bilayer phase diagram and study the thermodynamic, structural, and mechanical properties of a lipid membrane's fluid and gel phase, as well as of a polymer membrane's fluid phase in detail.

The second section, Sec. 3.2, focusses on the main phase transition, i.e., the transition between the fluid and a gel phase in a lipid bilayer. The precise location of the transition point is a formidable task, because it is obscured by hysteresis effects. We compare four different methods with different accuracies for locating it. Thereby we calculate the free energy along the path of a phase transformation from a fluid to a gel phase and learn how different sets of the involved degrees of freedom couple.

It has been conjectured that the lateral demixing of multi-component lipid bilayers into coexisting domains is actively used by cells to control the properties of the cell membrane. However, a quantification of this lateral heterogeneity is difficult due to the complexity of the phase diagram. The simplest system that shows such a lateral demixing is a single-component bilayer at phase coexistence. Thus, we continue the study of the main phase transition in Sec. 3.3, calculate the line tension between fluid and gel domains, and look at the shape transitions of these domains.

A large amount of the material covered in this chapter has been published in Ref. 178. 


\subsection{Phase diagram and phase properties}

The aim of the present section is to establish the thermodynamical, structural, and mechanical properties of our model. In the course of these calculations, we perform many validity checks on the model and obtain a comprehensive overview about its applicability. In particular, we investigate three crucial aspects: the self-assembly, the bilayer phase diagram, and the elasticity of bilayers.

In the first part, Sec. 3.1.1, we focus on our model's ability to self-assemble into various morphologies. It has been pointed out that the unassisted selfassembly to bilayers is a crucial property which every realistic coarse-grained model for lipid membranes should have [71]. The problems with models lacking this ability are twofold. On the one hand, preassembled bilayers, which are not the thermodynamically favored morphology, have to be used as initial configurations. Although the physical system is thereby trapped in a local minimum of the free energy, it might try to escape, e.g., by sporadically opening pores, by performing large undulations, or by ejecting micelles. Hence, nonphysical behavior might arise. On the other hand, such models do not yield true equilibrium averages, since they only sample a small, metastable region of the phase space.

The self-assembly process is also interesting for another reason. Depending on the interaction coefficients, the amphiphiles may self-assemble -besides just bilayers- into other morphologies like spherical, cylindrical, or wormlike micelles, as well as inverted structures with hydrophilic inclusions. The resulting morphology can be conceived as a direct consequence of the mean shape of the molecules and the way how they pack [3]. Thus, a careful study of the self-assembled morphologies provides a way of finding suitable interaction parameters for simulating bilayers.

In the second part, Sec. 3.1.2, we concentrate on bilayers and their different phases. A whole zoo of such phases have been identified experimentally and phase diagrams have been compiled for various synthetic and natural lipids, as well as lipid mixtures as function of temperature, pressure, water concentration, or composition [20, 179, and references therein]. Among other things there are a low-temperature subgel phase $\left(L_{C}\right)$, an interdigitated, non-tilted gel phase $\left(L_{\beta I}\right)$, a tilted gel phase $\left(L_{\beta^{\prime}}\right)$, a non-tilted gel phase $\left(L_{\beta}\right)$, a ripple phase $\left(P_{\beta^{\prime}}\right)$, and a fluid phase $\left(L_{\alpha}\right)$. The last phase is most important for cell biology, because cells are flexible, fluid-like objects and the normal thermodynamic state of the cell membrane is the fluid one.

With the increasing computing power over the last two decades, bilayer phase diagrams have also been obtained from computer simulations of atomistic and coarse-grained models [73, 75, 180-182]. Even though different variables, like the head-head repulsion, the range of attractive potentials, or the solvent den- 
sity, were used as variables for the phase diagrams, these simulations helped in clarifying the role individual lipid properties play for obtaining specific phases as well as the molecular structure of the gel phases at low temperatures $\left(<30^{\circ} \mathrm{C}\right)$. The molecular packing is dominated by the excluded volume of the tails, but the bulkiness of the head groups decides about the orientation of the lipids in the gel phases. If it is small, then the lipids arrange normal to the bilayer plane $\left(L_{\beta}\right)$, if it is large -as in the case of many natural lipids- they include a certain tilt angle with the bilayer plane $\left(L_{\beta^{\prime}}\right)$. If it is very large, the bilayer forms an interdigitated, non-tilted phase $\left(L_{\beta I}\right)$.

The ripple phase, $P_{\beta^{\prime}}$, which shows up in a narrow region between the $L_{\alpha}$ and the $L_{\beta^{\prime}}$ phase, has attracted particular interest because of its long wavelength modulation [75, 182, 183]. It could be demonstrated by computer simulations that this phase has a striped pattern with out-of-plane modulations in which fluid and tilted gel regions alternate. Its existence is a consequence of the stress induced in the bilayer that is caused by the spontaneous curvature of the monolayers that the $L_{\beta^{\prime}}$ phase is comprised of. This stress is, in turn, reduced by forming ripples.

In the third part of this section, Sec. 3.1.3 and 3.1.4, we turn to the mechanical and structural properties of the $L_{\alpha}$ and the $L_{\beta}$ phases. The bending rigidity, $\kappa$, of the fluid phases is the most important result in this context. It measures how much energy it costs to bend a bilayer, and its value is therefore crucial for determining the pathway of all phenomena in which bilayers have to deform, e.g., in pore formation and fusion, in undulations, or in pulling tethers from vesicles. Experimentally it is measured by one of two methods: either the thermal fluctuations of bilayer membranes are recorded and $\kappa$ is extracted from the power spectrum of these fluctuations, or the membrane is bent actively and the required force is measured [184-187]. Typical values of biomembranes are in the range of $\kappa=10-50 k_{B} T$ [188]. In computer simulations, $\kappa$ is routinely measured by the first method, i.e., from the power spectrum of fluctuations [70, 74, 181, 189-192], but an alternative employing an active deformation has also been proposed [193].

The area compression modulus, $K_{A}$, is a related quantity and is discussed in turn. It measures the energetic cost of a small stretching of a membrane and is frequently extracted from the area fluctuations. Experimental values are $\sim 240 \mathrm{mN} / \mathrm{m}$ for lipid bilayers [188] and $\sim 100 \mathrm{mN} / \mathrm{m}$ for polymeric membranes $[194,195]$. As a side product, we also obtain the mean area per lipid and, in combination with the density profile of the bilayer, the aspect ratio of the lipids, $f_{A}$. Finally, we map the simulation length scale to experimental units.

\subsubsection{Self-assembly}

In this subsection we study the lipids' self-assembly process from a random initial configuration to various morphologies. We use $N=16, R_{\mathrm{eo}}=3.5 r_{c}$, 
(a)

(c)
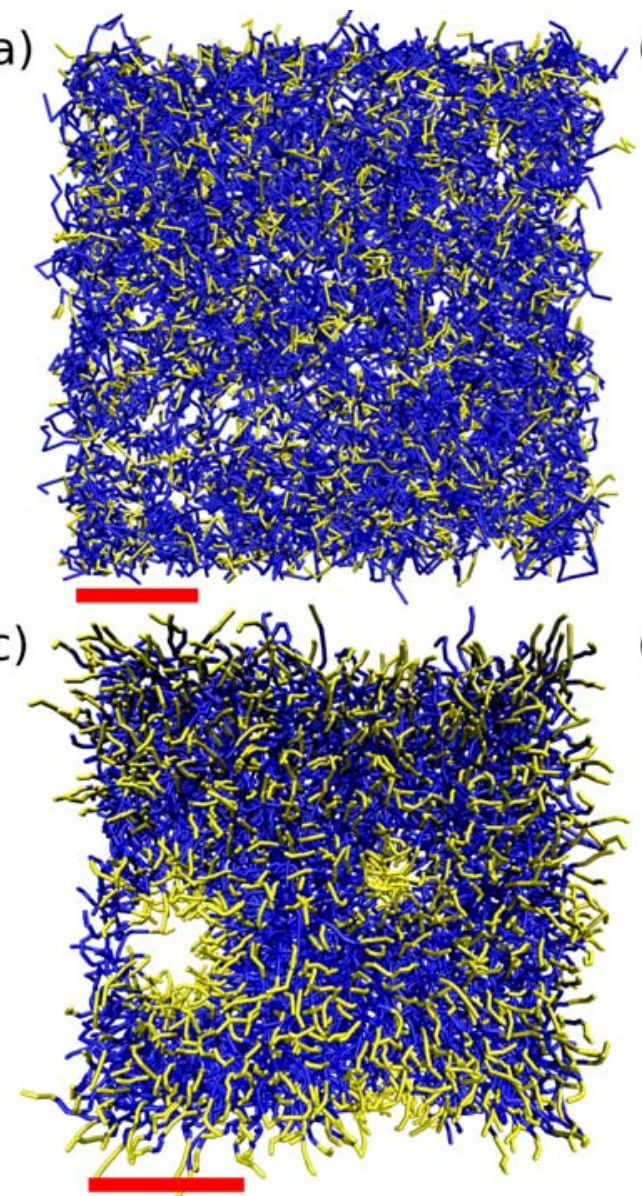

(b)

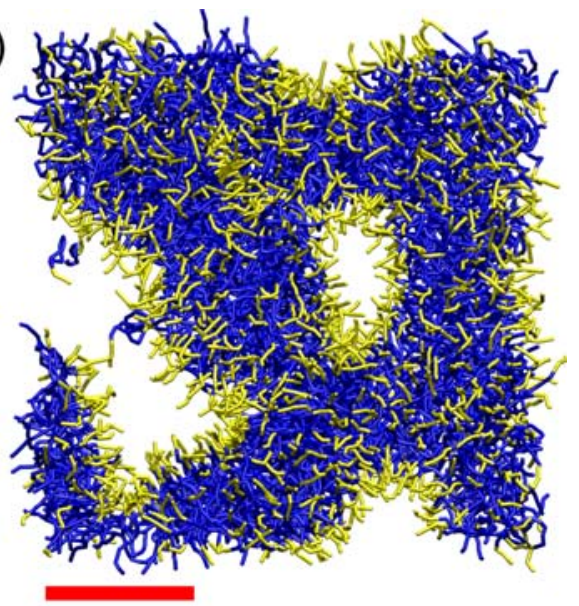

(d)

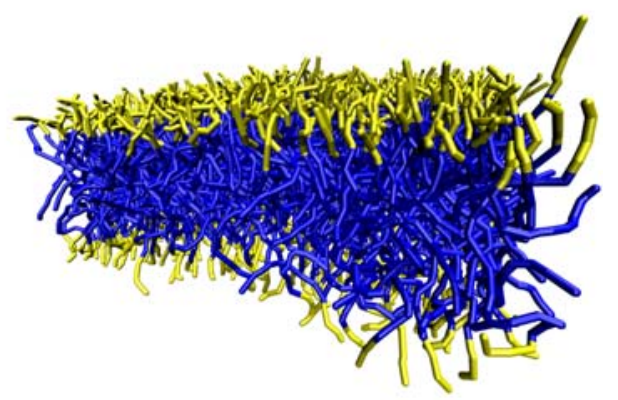

Figure 3.1: Self-assembly of the system $\bar{\rho}^{*}=17, \kappa N=100, \chi N=30, N_{A}=12$ (blue beads), and $N_{B}=4$ (yellow beads). (a) Initial configuration at $t=0$. (b) After $t=40 \tau$ broad micelles have formed. (c) At $t=200 \tau$ these micelles have coalesced to a bilayer with several pores. (d) After $t=800 \tau$ the pores have closed and a bilayer has been formed. The red scale bar denotes $7 r_{c}$. Reprinted with permission from Ref. 178. Copyright 2010, American Institute of Physics. 
and three different sets of $k_{s}$ and $k_{b}$, cf. Eq. (2.1): (i) $k_{s}=3.673$ and $k_{b}=0$ for flexible lipids without any bond-angle potential, (ii) $k_{s}=19.089$ and $k_{b}=5$ for lipids with a moderate stiffness, and (iii) $k_{s}=29.007$ and $k_{b}=10$ for lipids with a high stiffness. We perform all simulations in the $N P_{t} T$ ensemble and use an initial configuration comprised of $n=1600$ lipids in a box with $L_{x}=50 r_{c}$ and $L_{y}(t=0)=L_{z}(t=0)=30 r_{c}$. To avoid the formation of multiple bilayers, the lipids in the initial configuration were randomly distributed over the lower half of the box, i.e., $0<x_{i}<L_{x} / 2$. We explore various values of the coarse-grained parameters, $20 \leq \chi N \leq 100,50 \leq \kappa N \leq 500$, and $15 \leq \bar{\rho}^{*} \leq 40$, as well as different lengths of the hydrophobic tails, $N_{A}$, and of the hydrophilic heads, $N_{B}=N-N_{A}$.

Although the spatial extension of a lipid molecule is of the order $R_{\text {eo }}$, the fluctuations around this mean value are largely influenced by $k_{b}$. The conformations in the runs with $k_{b}=0$ correspond to fully flexible molecules and the shape fluctuations are of the same order of magnitude as the lipid's size, i.e., the conformations resemble a self-avoiding random walk. For $k_{b}=10$ the lipids are strongly elongated and they behave like rigid rods, which gives rise to a nematic, liquid crystalline structure of the self-assembled morphologies. Noteworthy, gel-like structures are only observed for $k_{b}=5,10$, whereas fluid like structures are only observed for $k_{b}=0,5$. Thus, the right usage of bond angle potentials is crucial for obtaining multiple bilayer phases. This observation is consistent with many other coarse-grained models where similar potentials are employed [63, 73, 74, 76].

The runs with $k_{b}=5$ showed the greatest spectrum of morphologies (see Tab. 3.1). Depending on the other parameters, the lipids self-assemble within $\Delta t \leq 500 \tau$ either into (i) spherical micelles, (ii) cylindrical micelles, (iii) wormlike micelles, (iv) small bilayers, (v) or bilayers with hydrophilic inclusions. Bilayers form for $N_{A} \geq 11$, and inverted structures, i.e., bilayers with hydrophilic inclusions, form for $N_{A} \geq 13$ (see Fig. 3.1). For $\chi N \geq 50$ wormlike or cylindrical micelles predominantly form, whereas for $\chi N<20$ the incompatibility between hydrophilic and hydrophobic beads becomes so small, that no clear separation between hydrophilic and hydrophobic regions is visible.

The observed sequence of morphologies is consistent with the geometrical arguments put forward by Israelachvili [3]. For $N_{A}<11$ the head groups are much larger than the tails and the amphiphiles prefer to assemble to micelles irrespective of the other parameters; their shape is in average conical. At $N_{A}=11$ the lipids form extended structures, like wormlike micelles or networks of these micelles, which indicates on average the form of a truncated cone. $N_{A}=12$ leads to bilayers, i.e., the lipids have a cylindrical shape. When the hydrophilic heads decrease in size, $N_{A}>12$, the molecules acquire the form of an inverted truncated cone and inverted morphologies appear. 
Table 3.1: Self-assembled morphologies obtained from a random initial configuration. The column headers in the right half denote the molecular asymmetry, e.g., 10/6 means $N_{A}=10$ and $N_{B}=6$. The morphologies are abbreviated: s=spherical micelles, $\mathrm{c}=$ cylindrical micelles, $\mathrm{w}=$ wormlike micelles, $\mathrm{b}=$ bilayers, $\mathrm{i}=$ inverted structures, i.e., bilayers with hydrophilic inclusions.

\begin{tabular}{rrrr|ccccccc}
\hline \hline $\bar{\rho}^{*}$ & $\kappa N$ & $\chi N$ & $k_{b}$ & $10 / 6$ & $11 / 5$ & $12 / 4$ & $13 / 3$ & $14 / 2$ & $15 / 1$ \\
\hline 15 & 100 & 40 & 0 & $\mathrm{c}$ & $\mathrm{b}$ & $\mathrm{b}$ & $\mathrm{i}$ & $\mathrm{i}$ & $\mathrm{i}$ \\
15 & 100 & 40 & 5 & $\mathrm{~s}$ & $\mathrm{~b}$ & $\mathrm{~b}$ & $\mathrm{~b}$ & $\mathrm{i}$ & $\mathrm{i}$ \\
15 & 100 & 60 & 0 & $\mathrm{~s}$ & $\mathrm{~b}$ & $\mathrm{~b}$ & $\mathrm{~b}$ & $\mathrm{i}$ & $\mathrm{i}$ \\
15 & 100 & 60 & 5 & $\mathrm{~s}$ & $\mathrm{~b}$ & $\mathrm{~b}$ & $\mathrm{i}$ & $\mathrm{i}$ & $\mathrm{i}$ \\
18 & 80 & 20 & 5 & $\mathrm{~s}$ & $\mathrm{w}$ & $\mathrm{w}$ & & & \\
18 & 80 & 30 & 5 & $\mathrm{~s}$ & $\mathrm{c}$ & $\mathrm{w}$ & & & \\
18 & 80 & 40 & 5 & $\mathrm{~s}$ & $\mathrm{c}$ & $\mathrm{w}$ & & & \\
20 & 100 & 20 & 5 & $\mathrm{~s}$ & $\mathrm{c}$ & $\mathrm{b}$ & $\mathrm{b}$ & & \\
20 & 100 & 30 & 5 & $\mathrm{~s}$ & $\mathrm{c}$ & $\mathrm{b}$ & $\mathrm{i}$ & & \\
20 & 100 & 40 & 5 & $\mathrm{~s}$ & $\mathrm{~s}$ & $\mathrm{c}$ & $\mathrm{b}$ & & \\
20 & 100 & 50 & 5 & $\mathrm{~s}$ & $\mathrm{~s}$ & $\mathrm{w}$ & $\mathrm{b}$ & & \\
\hline \hline
\end{tabular}

We conclude from these results, that $N_{A}=12, N_{B}=4, k_{b}=5$, and $k_{s}=19.089$ is a set of parameters that reproduces thermodynamically stable bilayers. Hence, we will use this set exclusively in the remainder of this work.

\subsubsection{Phase diagram}

Though the self-assembly runs have already provided a first glimpse on the possible bilayer phases, a more systematic study is needed for obtaining the full bilayer phase diagram of our model. To this end, we prepared an initial bilayer configuration in the fluid phase with $n=500$ molecules, quenched this configuration in many independent runs with $\kappa N=75 \ldots 150, \bar{\rho}^{*}=12 \ldots 40$, and $\chi N=30$ to the desired point in the bilayer phase diagram, and performed simulations for $500 \tau$ in the $\mathrm{NP}_{t} \mathrm{~T}$ ensemble. This procedure allowed for a quick overview of the phase diagram, but is seriously hampered by metastability effects near the phase boundaries. The result is shown in Fig. 3.2.

Four qualitatively different phases are detected: (i) the $L_{\alpha}$ phase, (ii) the $L_{\beta}$ phase, (iii) the $L_{\beta I}$ phase, and (iv) the $L_{\beta^{\prime}}$ phase, see Fig. 3.3. We note that Fig. 3.2 misses the ripple phase, $P_{\beta^{\prime}}$. It is known that this phase normally shows up in a narrow region between the $L_{\alpha}$ and the $L_{\beta^{\prime}}$ phase. Since we omitted a careful study of the $L_{\beta^{\prime}}$ phase, we probably overlooked the $P_{\beta^{\prime}}$ phase. Nevertheless, it is still possible that our model includes it. If it existed, it would probably be located at $\kappa N \geq 140$ and $\bar{\rho}^{*} \geq 20$. 


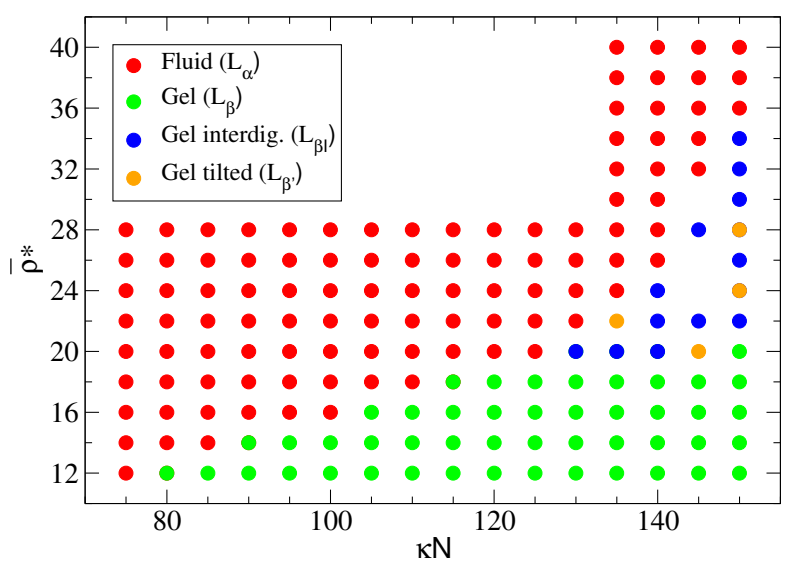

Figure 3.2: Phase diagram obtained by quenching an initial configuration in the fluid phase to the given $\kappa N$ and $\bar{\rho}^{*}$. Four, qualitatively different phases have been observed that are portraited in Fig. 3.3. Some simulations yielded bilayers with different phases on the two leaflets; in this case an empty spot is shown. Others contained coexisting domains of two phases; in this case the majority phase is shown.

With increasing $\bar{\rho}^{*}$ each coarse-grained bead interacts with more neighbors, so that the mean-field approximation becomes more accurate and fluid-like packing effects weaker. This marks the crossover to polymeric membranes, where the chain number density is typically higher than in lipid membranes and only a fluid phase is stable.

\subsubsection{Observables}

In this subsection we define the equilibrium quantities that will be used to characterize the phases. Since most of them are well known, their discussion is kept brief.

\section{Local structure}

Although scanning microscopes are becoming increasingly popular for obtaining real-space images of microscopic biological systems, most structural information is still obtained from scattering experiments, either with photons, neutrons, or electrons. To facilitate the comparison of our model bilayer's structure with experiments, we calculate the lateral static structure factor, $G(\mathbf{q})$. Since the scattering theory is covered in detail in many introductory textbooks to condensed matter physics [196-199], we are content with a brief derivation of the formulas needed for this work. Our presentation is based on Ref. 198, chap. 2.1. 

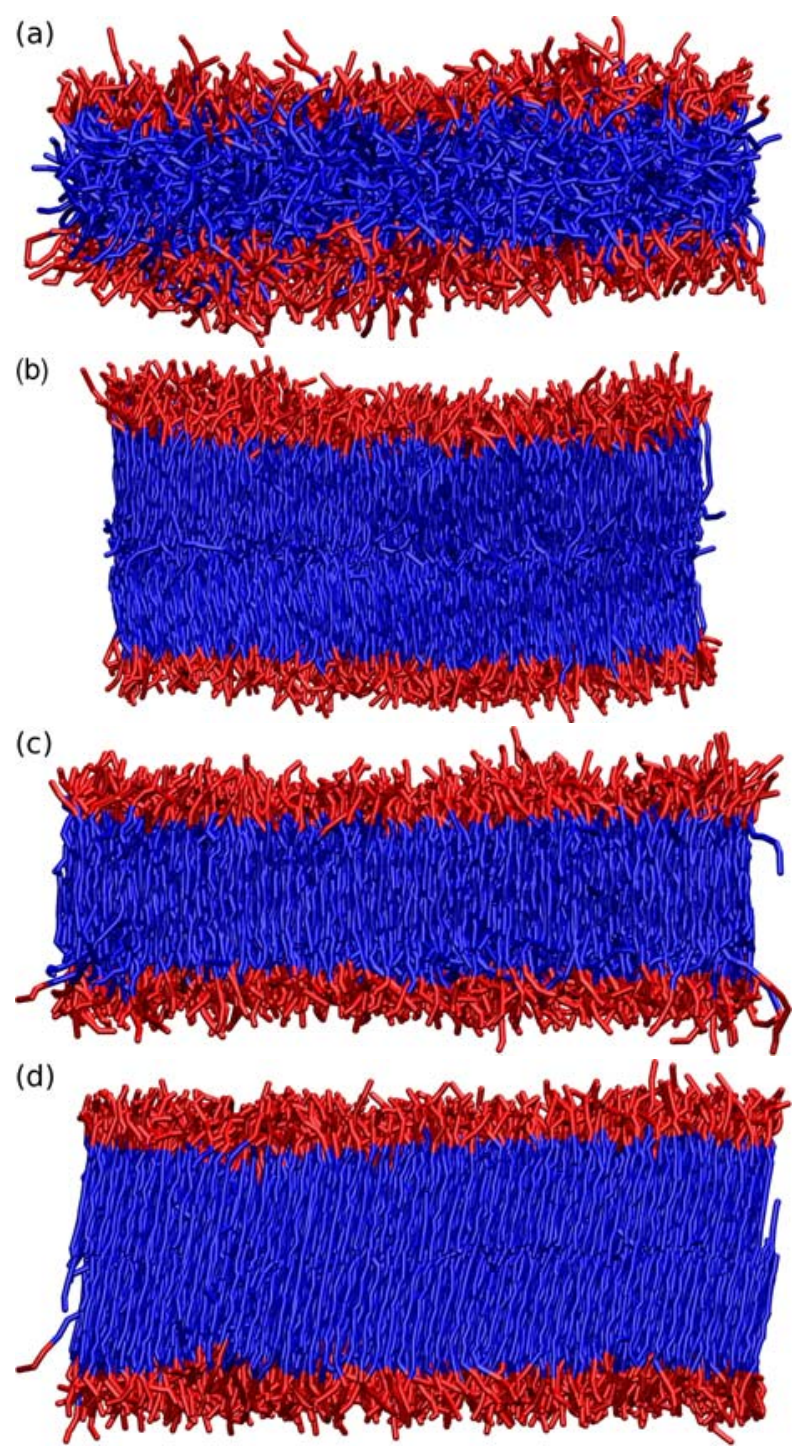

Figure 3.3: Images of the four phases (drawn to scale): (a) $L_{\alpha}$ fluid phase, (b) $L_{\beta}$ gel phase, (c) $L_{\beta I}$ interdigitated gel phase, (d) $L_{\beta^{\prime}}$ tilted gel phase. Head groups are shown in red, tails in blue. 
If an incident plane wave with wave vector $\mathbf{k}$ is scattered elastically by a sample to an outgoing wave vector $\mathbf{k}^{\prime}$, then the differential cross-section,

$$
\frac{d^{2} \sigma}{d \Omega} \sim \frac{2 \pi}{\hbar}\left|\left\langle\mathbf{k}|U| \mathbf{k}^{\prime}\right\rangle\right|^{2}
$$

is, according to Fermi's golden rule, proportional to the square modulus of the matrix element of the incoming and the outgoing plane wave state with the interaction potential of the sample, $U(\mathbf{r})$. If there are $N$ scatterers, located at $\mathbf{r}_{i}, i=1 \ldots N$, each having an interaction potential $U_{i}(\mathbf{r})$, then $U(\mathbf{r})$ may be written as

$$
U(\mathbf{r})=\sum_{i} U_{i}\left(\mathbf{r}-\mathbf{r}_{i}\right)
$$

An expansion of this matrix element yields after some algebra

$$
\left|\left\langle\mathbf{k}|U| \mathbf{k}^{\prime}\right\rangle\right|^{2}=\sum_{i, j} U_{i}(\mathbf{q}) U_{j}(-\mathbf{q}) e^{-i \mathbf{q} \cdot \mathbf{r}_{i}} e^{i \mathbf{q} \cdot \mathbf{r}_{j}}
$$

where $\mathbf{q}=\mathbf{k}-\mathbf{k}^{\prime}$ is the two-dimensional transferred momentum vector and $U_{i}(\mathbf{q})$, the Fourier transform of $U_{i}(\mathbf{r})$, the atomic form factor.

A special case arises, if all scatterers have the same atomic form factor. Then, $U_{i}(\mathbf{q})$ can be taken out of the sum, and the differential cross-section can be written as

$$
\frac{d^{2} \sigma}{d \Omega} \sim N\left|U_{i}(\mathbf{q})\right|^{2} G(\mathbf{q}),
$$

where we have introduced the static structure factor,

$$
G(\mathbf{q})=\frac{1}{N}\left\langle\sum_{i, j} e^{-i \mathbf{q} \cdot\left(\mathbf{r}_{i}-\mathbf{r}_{j}\right)}\right\rangle=\frac{1}{N}\left\langle\left|\sum_{i} e^{-i \mathbf{q} \cdot \mathbf{r}_{i}}\right|^{2}\right\rangle,
$$

which is intensive for all $\|\mathbf{q}\|>0 . G(\mathbf{q})$ is the sum of $N^{2}$ complex numbers with phases determined by all $N$ scatterers. If the relative positions, $\mathbf{r}_{i}-\mathbf{r}_{j}$, are randomly distributed, then destructive interference occurs, i.e., these numbers average to zero. If a periodicity with wavelength $\lambda$ exists, then the interference is constructive and a peak at the corresponding wave vector, $q=2 \pi / \lambda$, shows up in $G(\mathbf{q})$.

The numerical evaluation of $G(\mathbf{q})$ has been performed on a lattice, and the details of this calculation are given later at page 82. Due to the periodic boundary conditions, the wave vector, q, assumes only discrete values of the form $\mathbf{q}=2 \pi\left(n_{y} / L_{y}, n_{z} / L_{z}\right)$ with $n_{y}, n_{z} \in \mathbb{Z}$. 
The lateral pair correlation function,

$$
g(r)=\frac{1}{2 \pi r \Phi_{0} N}\left\langle\sum_{i=1}^{N} \sum_{j=1}^{N} \delta\left(r-\left\|\mathbf{r}_{j}-\mathbf{r}_{i}\right\|\right)\right\rangle,
$$

is a related quantity and measures correlations between two particles, that are separated in the plane by a distance $r$. The primed sum indicates that pairs with $i=j$ are excluded and $\Phi_{0}=N / L_{y} L_{z}$ is the particle number density. The normalization is chosen such that $g(r \rightarrow \infty) \rightarrow 1$.

\section{Density profile}

Stable fluid membranes in a solvent form a bilayer structure. The hydrophilic head groups on the outside favor contact with the solvent, and the tails constitute the bilayer's hydrophobic interior, which is shielded from the solvent. This lamellar structure becomes visible in the molecular density profile, which we record for the two leaflets separately. To avoid a broadening of these profiles by thermal undulations, we laterally subdivide the simulation box into small cells of a size $r_{c} \times r_{c}$. In each cell, the local bilayer position is determined and the profiles are averaged with respect to the local bilayer position over all cells and along a trajectory.

The hydrophobic interior's width, $w$, is estimated by measuring the full width at half maximum (FWHM) of the total hydrophobic density profile. The bilayer thickness, $t_{h}$, is estimated from the distance between the two peaks arising from the hydrophilic head groups on each leaflet.

\section{Bending rigidity}

The starting point for the calculation of the bending rigidity, $\kappa$, in a particlebased model is the Helfrich Hamiltonian [102-104],

$$
\mathcal{H}=\int d A\left(\frac{\kappa}{2}\left(2 H-C_{0}\right)^{2}+\kappa_{G} K+\gamma\right)
$$

which is an integral over the curved surface of the bilayer. Locally, the surface can be described by a symmetric curvature tensor with two eigenvalues, the inverse principal radii of curvature, $R_{1}^{-1}$ and $R_{2}^{-1}$. The two invariants of the tensor, the trace and the determinant, are denoted as the mean curvature, $H$, and the Gaussian curvature, $K$, respectively. They are defined at every point on the surface by

$$
H=\left(R_{1}^{-1}+R_{2}^{-1}\right) / 2 \text { and } \quad K=R_{1}^{-1} \times R_{2}^{-1} .
$$


$C_{0}$ is the spontaneous curvature of the bilayer, and $\kappa_{G}$ is the saddle-splay modulus. An in-depth discussion of this Hamiltonian and its terms is given by Seifert [105].

The first summand in Eq. (3.7) penalizes deviations of the actual curvature from the spontaneous curvature and the constant of proportionality is the bending rigidity. In the following we will focus on planar bilayers comprised of symmetric monolayers without such a spontaneous curvature, i.e., $C_{0}=0$, where the term penalizes any curvature. The second summand in the integral is, due to the Gauss-Bonnet theorem, a topological invariant, i.e., it is constant as long as the topology of the bilayer does not change. Its value is given by $\int d A K=4 \pi(1-g)$, where $g$ is the "genus" of the surface, i.e., the number of handles. It contributes only a constant shift to the energy which is neglected in the following. The third summand is the mechanical tension, $\gamma$, of the bilayer.

In this work, we focus on membranes that deviate only weakly from the plane, i.e., there are no overhangs. In this case the position of the bilayer midplane can be described in the Monge parameterization by a height, $h(\mathbf{r})$, above some reference plane. It can be shown that in the limit of a nearly flat surface [200],

$$
\mathcal{H}=\frac{1}{2} \int d^{2} r\left(\kappa\left(\nabla^{2} h\right)^{2}+\gamma(\nabla h)^{2}\right)+\text { const. }
$$

It is advantageous to expand $h(\mathbf{r})$ in a Fourier series, i.e., to write

$$
h_{\mathbf{q}}=\frac{1}{L_{y} L_{z}} \int d^{2} r h(\mathbf{r}) e^{-i \mathbf{q} \cdot \mathbf{r}} \quad h(\mathbf{r})=\sum_{\mathbf{q}} h_{\mathbf{q}} e^{i \mathbf{q} \cdot \mathbf{r}} .
$$

Insertion of Eq. (3.10) into Eq. (3.9) leads to an expression for $\mathcal{H}$,

$$
\mathcal{H}=\frac{L_{y} L_{z}}{2} \sum_{\mathbf{q}}\left|h_{\mathbf{q}}\right|^{2}\left(\kappa q^{4}+\gamma q^{2}\right)
$$

in which the different Fourier modes, $h_{\mathbf{q}}$, decouple. The energy density of each mode is given by the term in brackets. If the undulation modes are considered as the degrees of freedom of the bilayer, then the equipartition theorem may be invoked to obtain the well-known expression for the equilibrium power spectrum,

$$
\left\langle\left|h_{\mathbf{q}}\right|^{2}\right\rangle=\frac{k_{B} T}{L_{y} L_{z}\left(\kappa q^{4}+\gamma q^{2}\right)} .
$$

Equation (3.12) allows us to obtain $\kappa$ from particle-based simulations, if the power spectrum is measured. The calculation of $\left\langle\left|h_{\mathbf{q}}\right|^{2}\right\rangle$ is done in two steps. First, we define a linear, two-dimensional mesh with $16 \times 16$ grid points over the area of the bilayer. The bilayer's height at each grid point is determined in every 
snapshot by the average normal coordinate of the surrounding hydrophobic beads. In a second step, we calculate a two-dimensional, discrete fast Fourier transform to obtain $h_{\mathbf{q}}$ and $\left|h_{\mathbf{q}}\right|^{2}$. It has been pointed out by Cooke and Deserno that this way of assigning beads to grid points introduces spectral artifacts which must be accounted for [181]. We do this by dividing $\left|h_{\mathbf{q}}\right|^{2}$ through

$$
\operatorname{sinc}^{2}\left(\frac{n_{y}}{16}\right) \operatorname{sinc}^{2}\left(\frac{n_{z}}{16}\right),
$$

where $n_{y}=q_{y} L_{y} / 2 \pi$ and $n_{z}=q_{z} L_{z} / 2 \pi$. Finally, we obtain $\left\langle\left|h_{\mathbf{q}}\right|^{2}\right\rangle$ by averaging over many snapshots.

\section{Area compressibility}

The area compressibility, $k_{A}$, of a bilayer is defined as

$$
k_{A}=\frac{1}{A} \frac{\partial A}{\partial \gamma}
$$

where $\gamma$ is the mechanical tension and $A$ is the surface area of the bilayer. In this work, we adopt the common approximation of identifying $A$ with the projected area, i.e., the lateral area of the simulation box, $L_{y} \times L_{z} \cdot{ }^{1}$ The approximation renders the calculation of $k_{A}$ in the $\mathrm{NP}_{t} \mathrm{~T}$ ensemble, where $A(t)$ fluctuates, a straightforward task. The partition function for this ensemble [127],

$$
\mathcal{Z}_{N \gamma T}=\int_{0}^{\infty} d A e^{\beta \gamma A} \mathcal{Z}_{N V T}(A),
$$

depends on $\gamma$ only through the first, exponential factor; the canonical partition function, $\mathcal{Z}_{N V T}$, is independent of it. We compute $k_{A}$ by taking the second derivative of $\ln \left(\mathcal{Z}_{N \gamma T}\right)$ and obtain

$$
\begin{aligned}
\frac{\partial \ln \mathcal{Z}_{N \gamma T}}{\partial \gamma} & =\beta\langle A\rangle, \\
\frac{\partial^{2} \ln \mathcal{Z}_{N \gamma T}}{\partial \gamma^{2}} & =\beta \frac{\partial\langle A\rangle}{\partial \gamma}=\beta^{2}\left\langle A^{2}\right\rangle-\beta^{2}\langle A\rangle^{2} .
\end{aligned}
$$

Thus, with Eq. (3.15) we find

$$
k_{A}=\frac{1}{\langle A\rangle} \frac{\partial\langle A\rangle}{\partial \gamma}=\beta \frac{\left\langle A^{2}\right\rangle-\langle A\rangle^{2}}{\langle A\rangle} .
$$

\footnotetext{
${ }^{1}$ This neglects the excess area introduced by undulations and leads to a dependency of $k_{A}$ on the system size [201].
} 
Below, in Sec. 4.2, we will use the monolayer area compressibility, $k_{m}$. For the sake of simplicity we define $k_{m}=k_{A} / 2$.

\subsubsection{Phase properties}

In this subsection we study the properties of the $L_{\alpha}$ and the $L_{\beta}$ phase. To this end we simulate larger systems with $n=4680$ molecules in the $\mathrm{NP}_{t} \mathrm{~T}$ ensemble. Specifically, we investigate preassembled bilayers with three different densities, $\bar{\rho}^{*}=17,18$, and 40 . The first two have a low molecular density, i.e., they mimic lipid systems, whereas the latter one has a higher density and mimics a polymeric system. We use $\kappa N=100$ and $\chi N=30$.

\section{Local structure}

We start the discussion by inspecting the static structure factors, $G(\mathbf{q})$, of the $L_{\alpha}$ and $L_{\beta}$ phases in Fig. 3.4. The first one has a broad ring at $q r_{c} \approx 9.3$ and a peak at $q r_{c} \approx 0.33$. The ring is typically found in liquids and indicates that the molecules are distributed isotropically with an average separation of $\Delta x_{1} \approx 0.68 r_{c}$ and its width is related to the spread of molecular separations. In contrast to experimental diffraction patterns we miss, of course, the second microscopic length scale that corresponds to the separation of the two tails of a single molecule. The peak at small $q r_{c}$ indicates the existence of another, much larger length scale, $\Delta x_{2} \approx 19 r_{c}$. However, its origin was not analyzed in this work, but we expect that it originates from either large undulations or peristaltic waves.

The diffraction pattern of the $L_{\beta}$ phase has six sharp peaks oriented on a hexagon at $q r_{c} \approx 9.3$. They are surrounded by a background which rises up to $2 \%$ of the peak's height. From the existence of this background we tend to conclude that the $L_{\beta}$ phase has no hexagonal crystalline, but a hexatic, sixfold orientational order. Thus, there is a long-range orientational, but no long-range positional order. Interestingly, the peak at small $q$ does not show up in the $L_{\beta}$ phase for the system sizes studied.

The investigation of the bilayer structure is complemented by the calculation of the lateral pair correlation function between two head beads (B) and between two tails beads (A), in the same monolayer, $g_{\text {intra }}(r)$, as well as in opposite monolayers, $g_{\text {inter }}(r)$ (see Fig. 3.5). Unlike simple liquids, where the particles' repulsion creates a pronounced correlation hole at small $r, g_{\text {intra }}(r)$ is in our model always greater than unity at $r=0$. This is expected, because the lipids are oriented perpendicular to the plane where $g_{\text {intra }}(r)$ is calculated, so that the beads seem to overlap in the lateral pair correlation function. The hydrophobic beads in the same leaflet show weak packing effects up to the fourth coordination sphere in the fluid phase, and long range packing effects 

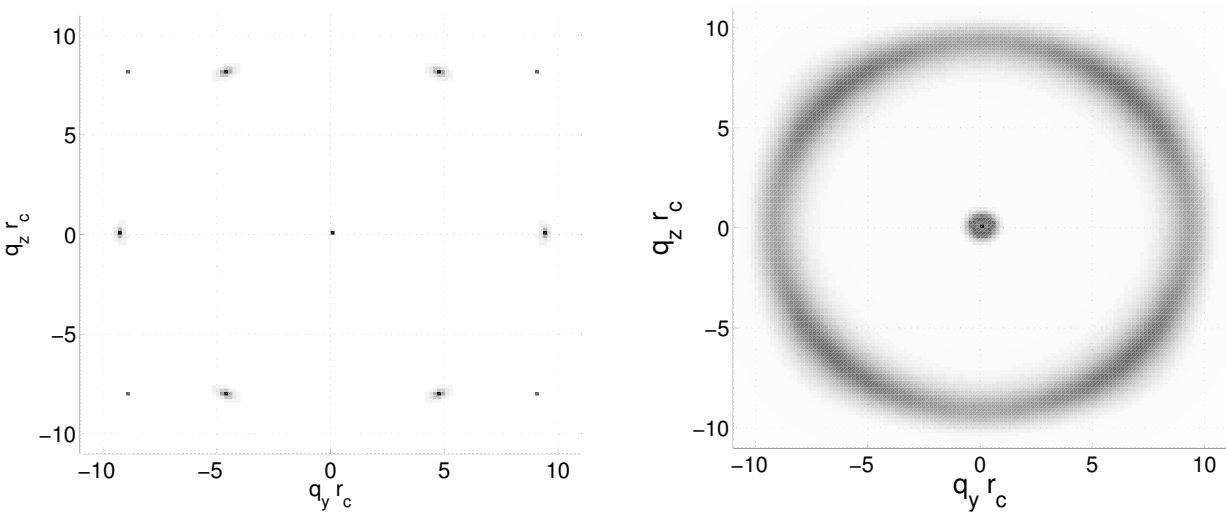

Figure 3.4: Static structure factor, $G(\mathbf{q})$, of the $L_{\beta}$ phase at $\bar{\rho}^{*}=17$ (left) and the $L_{\alpha}$ phase at $\bar{\rho}^{*}=18$ (right). The slight anisotropy of both patterns, as well as the four dots on a square in the left panel are numerical artifacts caused by the evaluation of $G(\mathbf{q})$ on a lattice.

in the gel phase. The pair correlation functions of the head groups look very similar in both phases, which is also expected, since the interactions are the same. The head beads of the same molecule overlap at small $r$, but they are almost uncorrelated with the head beads from other molecules, i.e., $g_{\text {intra }}(r)$ decays quickly to unity.

Even though the interactions between two head beads are purely repulsive $\left(v_{B B}=0.1, w_{B B B}=0\right)$ and the weighting function, $w_{2}(r)$, has negative Fourier components, there is no problem of running into a cluster-crystalline phase. It can be shown that the critical density for getting cluster crystals is $\rho=$ $N^{2} / v_{B B} R_{\mathrm{eo}}^{3}\left|\tilde{w}_{2}\left(k^{*}\right)\right| \approx 700 r_{c}^{-3}$, which is much larger than the actual density [135]. Here, $\tilde{w}_{2}(k)$ is the Fourier transform of $w_{2}(r)$ and $k^{*}$ is the wave number at which $\tilde{w}_{2}(k)$ attains its negative minimum.

The tail-tail pair correlation function between opposite leaflets has a small correlation hole. Its existence indicates that lipids on opposite leaflets tend to avoid each other. This effect is less pronounced in the fluid phase than in the gel phase, where $g_{\text {inter }}(0)$ falls to 0.38 . It means that the molecules in the gel phase on the one leaflet tend to reside in the interstitials of those on the other leaflet.

Finally we remark that the head groups on different leaflets are uncorrelated and that head-tail correlations are, even in the same leaflet, negligible and therefore omitted. 


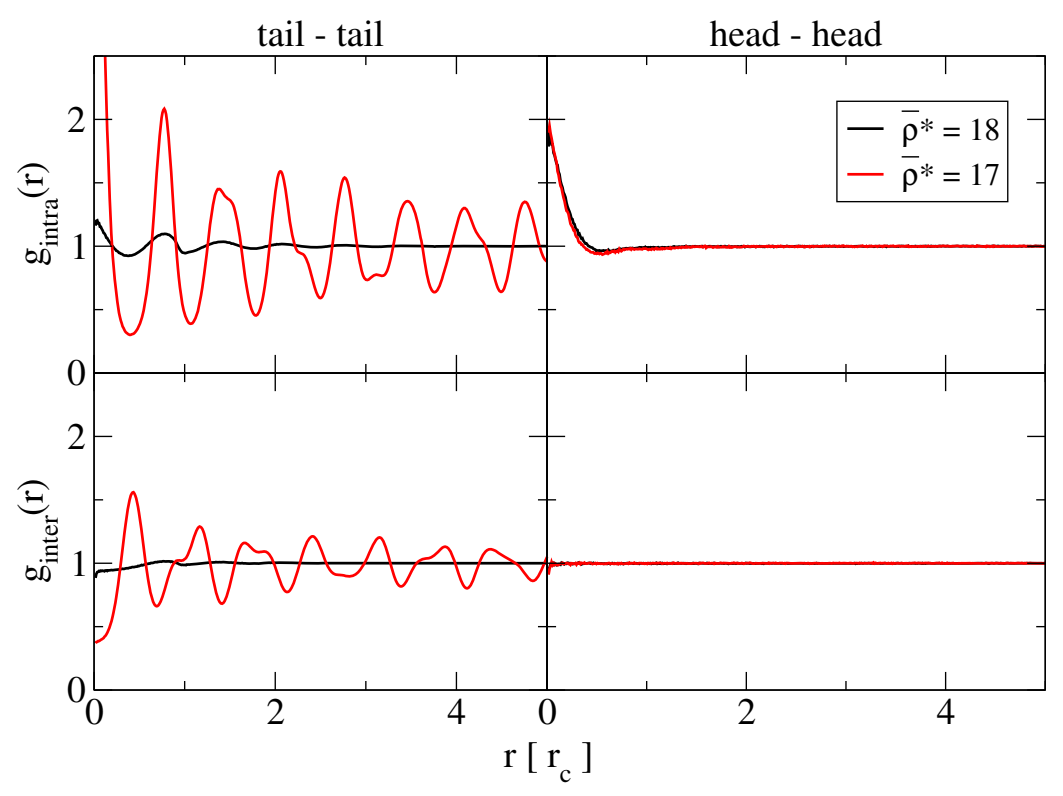

Figure 3.5: Top row: lateral pair correlation function within the same leaflet between two tail beads (left column) and between two head beads (right column). Bottom row: lateral pair correlation function between beads in different leaflets.

\section{Density profiles and conversion factors}

Figure 3.6 depicts three density profiles, $\hat{\rho}_{\alpha}(x)$, across the bilayer. They belong to the $L_{\beta}$ phase at $\bar{\rho}^{*}=17$, the $L_{\alpha}$ phase at $\bar{\rho}^{*}=18$, and the $L_{\alpha}$ phase at $\bar{\rho}^{*}=40$. All three show separated peaks for the hydrophilic heads and the hydrophobic tails which implies that the amphiphiles indeed form bilayer membranes. A closer inspection of the densities of the hydrophobic interior shows that the two leaflets are clearly distinguishable, but that there is no dip in the center of the density profile, like it is known from atomistic or systematically coarse-grained models including solvent [202, 203]. In fact, we find a flat profile in the fluid phase and a minor hump in the gel phase, the latter being caused by a small overlap of the last bead of the lipids from each side. We find $w=5.60 r_{c}$ and $t_{h}=7.07 r_{c}$ for $\bar{\rho}^{*}=17, w=4.93 r_{c}$ and $t_{h}=6.45 r_{c}$ for $\bar{\rho}^{*}=18$, and $w=4.25 r_{c}$ and $t_{h}=5.77 r_{c}$ for $\bar{\rho}^{*}=40$.

This overlap might arise from three different reasons. (i) The molecular shape in our model is finely discretized and the tails are rather flexible. If it becomes more rod-like, the density profile at the center develops a dip due to the molecular packing. This could be achieved by a decrease of the number of beads per lipid or an increase of the bond stiffness. (ii) If the incompatibility between hydrophobic and hydrophilic segments increases, the bilayer thickness also increases and the interdigitation between the opposite leaflets decreases. 


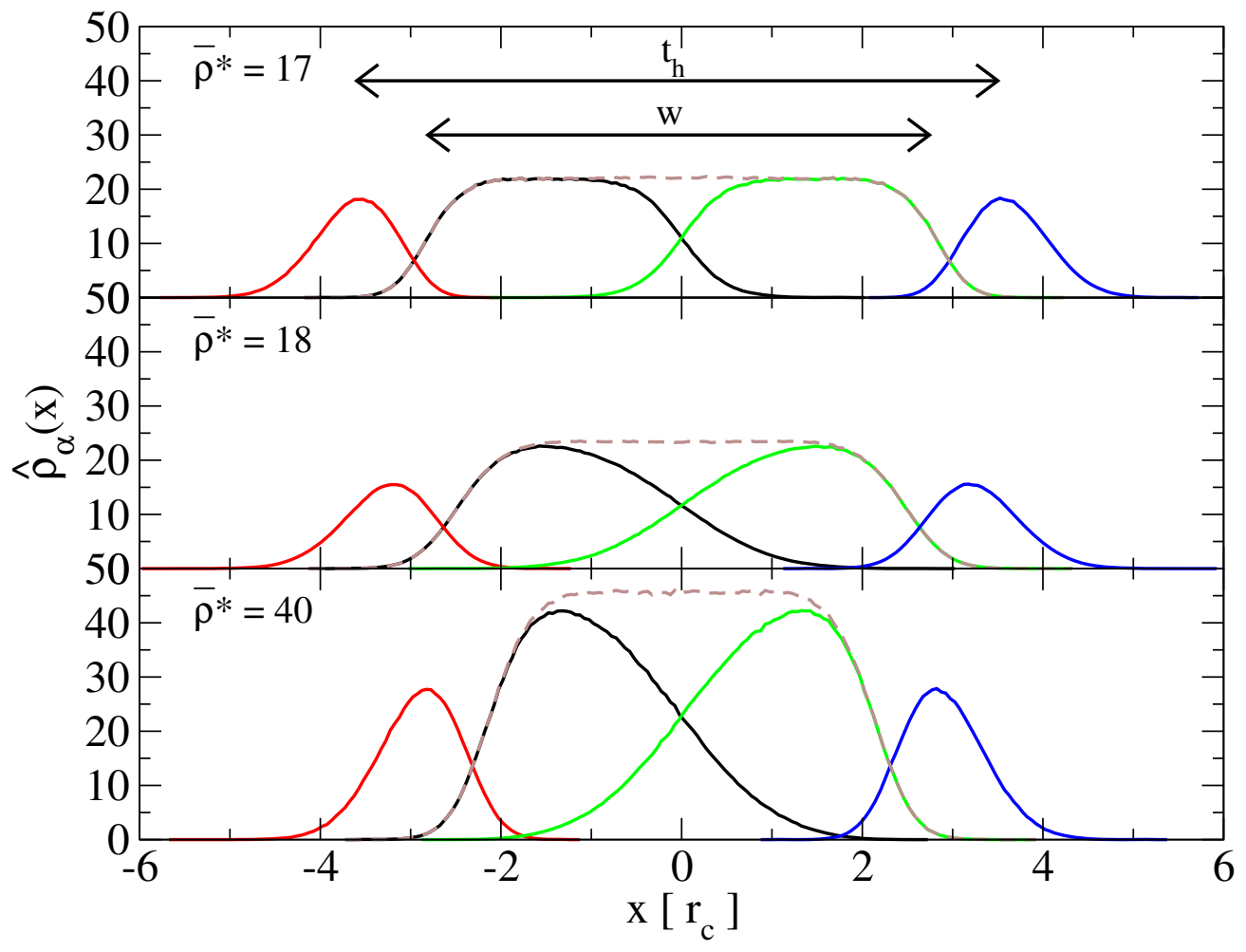

Figure 3.6: Density profiles of the $L_{\beta}$ phase at $\bar{\rho}^{*}=17$ (top), and the $L_{\alpha}$ phase at $\bar{\rho}^{*}=18$ (middle) and $\bar{\rho}^{*}=40$ (bottom). The densities of the head beads in both leaflets are colored in red and blue, and the densities of the tail beads in black and green, respectively. The sum of the tail densities is marked by the dashed, gray line. As noted above, packing effects lead to a deviation of the hydrophobic interior's density from the estimate of the coexistence density, $\bar{\rho}^{*}$, provided by mean-field theory. 
(iii) The flat density profile could also arise from the lack of solvent molecules. Since there is no solvent exerting pressure on the membrane, the lipids might have to interdigitate slightly so that the whole bilayer remains stable.

The average area per lipid, $\langle a\rangle=2\langle A\rangle / n$, is an important parameter in the study of the lipid bilayer phases. We obtained $\langle a\rangle=0.52 r_{c}^{2}, 0.56 r_{c}^{2}$, and $0.33 r_{c}^{2}$ for $\bar{\rho}^{*}=17,18$, and 40 , respectively.

Although $w$ and $\langle a\rangle$ depend on the details of the underlying model, the dimensionless aspect ratio, $f_{A}=w / \sqrt{\langle a\rangle}$, can straightforwardly be compared to experiments. The most common two-tailed lipids have aspect ratios in the range of $f_{A} \approx 3-5$, whereas our single-tailed model lipids have $f_{A} \approx 6-7$. If we assumed that two of our single-tailed lipids glued together result in one two-tailed lipid, we would double the mean area per lipid, $\langle a\rangle$, and obtain an additional factor of $\sqrt{2}$ in the denominator of $f_{A}$. Thereby we would obtain aspect ratios that are in good agreement with experimental values. Noteworthily, it is impossible to obtain the right aspect ratio simply by selecting different interaction coefficients at fixed molecular architecture and fixed discretization, $N$.

To establish a conversion factor between the unit of length in simulations and in experiments, we use the thickness of the bilayer, $t_{h}=6.45 r_{c}$. From synthetic lipids like DPPC, DPPE, or DLPC it is known that $t_{h} \approx 5 \mathrm{~nm}$. Hence, we obtain for the lipidic system $\left(\bar{\rho}^{*}=18\right)$

$$
1 r_{c}=0.78 \mathrm{~nm}
$$

which is also in good agreement with the typical size of coarse-grained beads in models for alkanes. For the polymeric system, $\bar{\rho}^{*}=40$, the thickness, $t_{h}=5.77 r_{c}$, is typically between 10 and $20 \mathrm{~nm}$. Thus we get,

$$
1 r_{c}=2.6 \mathrm{~nm}
$$

\section{Elastic properties}

Figure 3.7 shows the undulation power spectrum of tensionless bilayers in the $L_{\alpha}$ phase at $\bar{\rho}^{*}=18$ and 40 . By fitting $k_{B} T / A\left\langle\left|h_{\mathbf{q}}\right|^{2}\right\rangle q^{2}$ as a function of $q^{2}$ to a line through the origin, we extract $\kappa$ from the slope, cf. Eq. (3.12). We obtain $\kappa=20.1(4) k_{B} T$ and $\kappa=19.7(4) k_{B} T$ for $\bar{\rho}^{*}=18$ and 40, respectively. These values lie within the interval of experimental relevance for lipidic as well as polymeric membranes. Thus, our model is able to describe realistic deformations of the bilayer. Since the various gel phases are essentially rigid, there are no undulations and, consequently, no bending rigidity can be obtained by this method. 


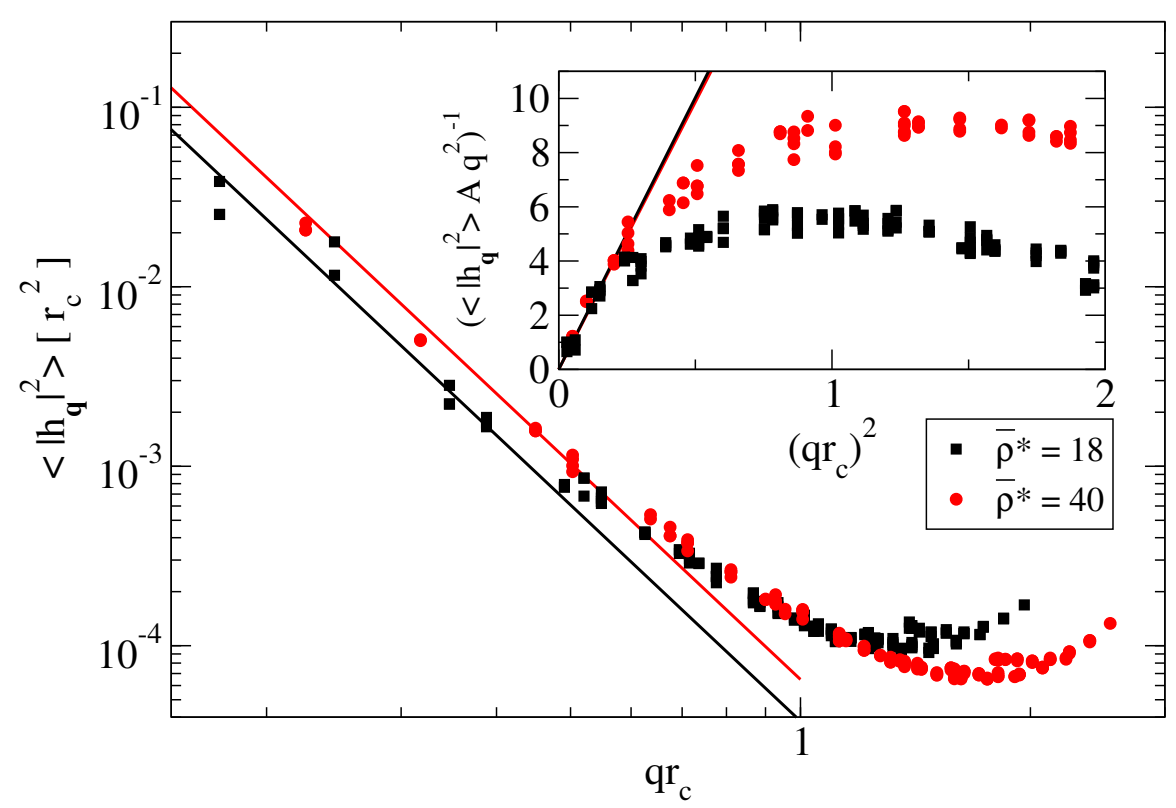

Figure 3.7: Undulation power spectrum of the fluid phase for $\bar{\rho}^{*}=18$ (black) and $\bar{\rho}^{*}=40$ (red). The solid lines show a fit to the asymptotic $q^{-4}$ power law, cf. Eq. (3.12). Inset: $\left(\left\langle\left|h_{\mathbf{q}}\right|^{2}\right\rangle A q^{2}\right)^{-1}$ shown as a function of $q^{2} . \kappa$ is given by the slope of the line in the limit $q \rightarrow 0$. Reprinted with permission from Ref. 178. Copyright 2010, American Institute of Physics.

We measured $k_{A}$ according to Eq. (3.18) for the $L_{\beta}$ phase at $\bar{\rho}^{*}=17$ as well as for the $L_{\alpha}$ phase at $\bar{\rho}^{*}=18$ and 40 , and obtained $k_{A}=1.68 \times 10^{-3} r_{c}^{2} / k_{B} T$, $k_{A}=0.46 r_{c}^{2} / k_{B} T$, and $k_{A}=0.051 r_{c}^{2} / k_{B} T$, respectively. We used the conversion factor from Eq. (3.19) to convert $k_{A}$ for the lipidic systems, $\bar{\rho}^{*}=17$ and 18 , to area compression moduli, $K_{A}=k_{A}^{-1}$, in experimental units and obtained $K_{A}=3960 \mathrm{mN} / \mathrm{m}$ in the $L_{\beta}$ phase and $K_{A}=14.4 \mathrm{mN} / \mathrm{m}$ in the $L_{\alpha}$ phase. Hence, the area fluctuations in our model are more pronounced than in experiments, where $K_{A} \approx 240 \mathrm{mN} / \mathrm{m}$ ( $L_{\alpha}$ phase) [188]. For the polymeric system, $\bar{\rho}^{*}=40$, we used Eq. (3.20) for the conversion and obtained $K_{A}=11.7 \mathrm{mN} / \mathrm{m}$, which is an order of magnitude smaller than in experiments, $K_{A} \approx 100 \mathrm{mN} / \mathrm{m}$ $[194,195]$. We attribute these larger fluctuations to the lack of harsh repulsions.

\subsubsection{Summary}

In this section we have demonstrated that our model captures three crucial properties: it self-assembles into bilayers, offers a rich bilayer phase diagram, and has a high bending rigidity. Further, due to the universality of the phenomena, it can be used to describe polymeric as well as lipidic bilayers. 
Table 3.2: Summary of some important static bilayer properties

\begin{tabular}{lllccc}
\hline \hline Quantity & Symbol & Unit & $\bar{\rho}^{*}=17$ & $\bar{\rho}^{*}=18$ & $\bar{\rho}^{*}=40$ \\
\hline Bilayer phase & & & $L_{\beta}$ & $L_{\alpha}$ & $L_{\alpha}$ \\
Area & $\langle A\rangle$ & $r_{c}^{2}$ & 1222 & 1304 & 780.2 \\
Area compressibility & $k_{A}$ & $r_{c}^{2} / k_{B} T$ & 0.168 & 46 & 5.1 \\
& & $\times 10^{-2}$ & & & \\
Area per lipid & $\langle a\rangle$ & $r_{c}^{2}$ & 0.52 & 0.56 & 0.33 \\
Hydrophobic width & $w$ & $r_{c}$ & 5.60 & 4.93 & 4.25 \\
Bilayer thickness & $t_{h}$ & $r_{c}$ & 7.07 & 6.45 & 5.77 \\
Aspect ratio & $f_{A}$ & 1 & 7.8 & 6.6 & 7.4 \\
Bending rigidity & $\kappa$ & $k_{B} T$ & $\ldots$ & $20.1(4)$ & $19.7(4)$ \\
\hline \hline
\end{tabular}

First, we analyzed the correlation between the self-assembled morphologies and the interaction parameters. Spherical, cylindrical, and wormlike micelles are obtained for large head groups; bilayers and inverted structures with hydrophilic inclusions are obtained for small head groups or small incompatibilities between hydrophilic and hydrophobic beads. The bond-angle stiffness, $k_{b}$, crucially determines the structure of bilayer phases: if its value is too low, only fluid bilayers are observed, if it is too high, only gel bilayers are observed. In the remainder of this thesis, we exclusively use $N=16, N_{A}=4, R_{\mathrm{eo}} / r_{c}=3.5$, $k_{s}=19.089$, and $k_{b}=5$.

Second, we compiled the approximate bilayer phase diagram as a function of $\bar{\rho}^{*}$ and $\kappa N$ by quenching an initial configuration to the desired state. Although the obtained phase diagram suffers from metastabilities in the vicinity of phase boundaries, four different phases were identified: the fluid phase, $L_{\alpha}$, the gel phase, $L_{\beta}$, the tilted gel phase, $L_{\beta^{\prime}}$, and an interdigitated phase, $L_{\beta I}$. No ripple phase, $P_{\beta^{\prime}}$, was observed.

Third, we focussed on $\kappa N=100$ and $\chi N=30$, where only the $L_{\alpha}$ and the $L_{\beta}$ phase are stable, and calculated many static properties. Among them are the the static structure factor, the bending rigidity, the density profile, and the area compressibility. The area compressibility is higher than in experimental systems, but this can be related to the softness of our model, where density fluctuations are more pronounced.

The most important results are summarized in Tab. 3.2.

\subsection{Main phase transition}

The phase diagram of single-component lipid bilayers comprises several gel and subgel phases, e.g., $L_{\beta}, L_{\beta^{\prime}}, L_{\beta I}, L_{C}$, the ripple phase, $P_{\beta^{\prime}}$, and the fluid phase, $L_{\alpha}$. The phase transitions between these phases have been experimentally 
studied in detail and they all seem to be discontinuous [20, and references therein]. Some of them acquired their own names, e.g., the "subtransition" $\left(L_{C} \leftrightarrow L_{\beta^{\prime}}\right)$, the "pretransition" $\left(L_{\beta}^{\prime} \leftrightarrow P_{\beta^{\prime}}\right)$, or the "main phase transition" $\left(P_{\beta^{\prime}} \leftrightarrow L_{\alpha}\right.$ or $\left.L_{\beta} \leftrightarrow L_{\alpha}\right)$

At high temperatures the bilayers are always in the $L_{\alpha}$ phase, but depending on the bulkiness of the head group, the phase diagrams differ at low temperatures qualitatively. Two different cases are distinguished. If the head group is small, like in the phosphatidylethanolamines $(\mathrm{PE})$, the lipids arrange at low temperatures in the non-tilted $L_{\beta}$ phase, where the director is parallel to the normal vector of the membrane. The main phase transition, $L_{\beta} \leftrightarrow L_{\alpha}$, does not involve an intermediate ripple phase. If it is large, like in the phosphatidylcholines (PC), the lipids arrange at low temperatures in a tilted phase, $L_{\beta^{\prime}}$, with a certain angle between the director and the normal vector. When the temperature is increased, the pretransition into the ripple phase $\left(L_{\beta}^{\prime} \leftrightarrow P_{\beta^{\prime}}\right)$ occurs first, followed by the main phase transition $\left(P_{\beta^{\prime}} \leftrightarrow L_{\alpha}\right)$ [204, and references therein].

In both cases the main phase transition simultaneously involves a change in the in-plane degrees of freedom that dictate the orientational order and a change in the conformational degrees of freedom [110]. The simultaneous transition in these two different sets of degrees of freedom can be decoupled by mixing the lipids with cholesterol [205]. In this case an intermediate phase, the liquid-ordered phase, in which the conformational degrees are still frozen, but the orientational degrees are unlocked, shows up. It is interesting to note that the main phase transition happens for most lipids in the vicinity of the body temperature, e.g., $41^{\circ} \mathrm{C}$ for DPPC. Hence, it is tempting to speculate that the proximity of this phase transition is used in some way or other in biology (see for instance Fig. 1.2 or Fig. 3.8).

Early simulation studies of these phase transitions involved Ising-like, lattice models [108, 109], where the response functions have been used to locate phase transitions. Qualitative insights into the topology of the phase diagrams were obtained later by continuum models [206-208], but it was difficult to gain insights on the molecular scale with these models. Coarse-grained, off-lattice models are suited very well for the study of phase transitions, because they are - unlike atomistic models- able to incorporate a large number of molecules, so that finite-size effects are suppressed significantly [81, and references therein]. Due to their infinite number of conformations, they offer the possibility to study even miniscule changes in these degrees of freedom. Though various phase transitions have been observed in these coarse-grained models, no careful determination of the exact phase transition point has been presented so far. Most studies were content with an approximate location of the transition point by looking for hysteresis effects [71, 75]. Further it seems, that the situation of large head groups is of major relevance for cell biology, so that most studies 

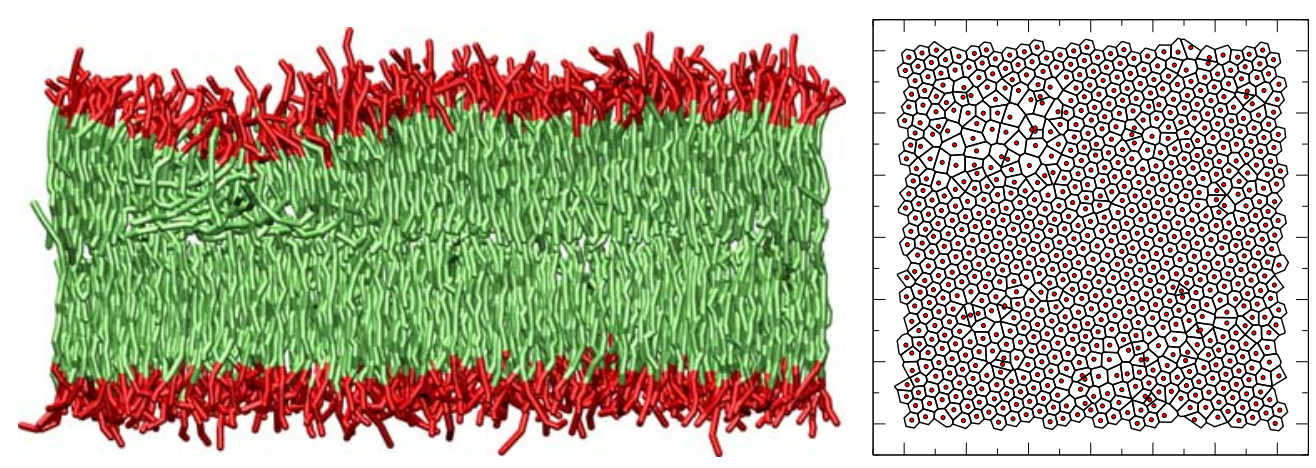

Figure 3.8: In the vicinity of the main phase transition metastable domains of the minority phase occur. The left panel shows a fluid domain $\left(L_{\alpha}\right.$; located in the upper left corner) embedded in the $L_{\beta}$ phase near coexistence. The right panel shows a Voronoi tesselation of the upper leaflet of the same configuration. The fluid domain can be clearly distinguished from the gel; the dots denote the centers of mass of the lipids.

focussed on the more complex situation where a pretransition occurs. The simpler main phase transition, $L_{\beta} \leftrightarrow L_{\alpha}$, without a preceding pretransition and a ripple phase received less attention in the literature.

Motivated by these facts, we focus on the somewhat simpler situation and locate the main phase transition, $L_{\beta} \leftrightarrow L_{\alpha}$, in our model precisely. In contrast to most other studies, we do not use the temperature as the control parameter to drive the transition, but the molecular density, $\bar{\rho}^{*}$. We use three different but not independent methods - to locate the phase coexistence. First, we apply a combination of umbrella sampling (US) and the weighted histogram analysis method (WHAM) [209-213] to compute the free energy, $F\left(\bar{\rho}^{*}\right)$, in the vicinity of the main phase transition. Second, we utilize the free energy perturbation theory (FEP) to extrapolate the free energy branches of each phase [213] and to find the value of $\bar{\rho}^{*}$ at which the branches intersect. Finally, we use a histogram reweighting scheme to calculate the specific heat, $C\left(\bar{\rho}^{*}\right)$.

In Sec. 3.2.1 we briefly explain the technical details of the free energy calculation and the reweighting methods. This presentation is based on the work by Souaille and Roux in Ref. 212. We apply them in Sec. 3.2.2 to our model and conclude the discussion of the main phase transition in Sec. 3.2.3.

\subsubsection{Free energy calculations}

\section{Order parameters}

Several order parameters can be used to characterize the various bilayer phases [80, 181, 203]. We chiefly employ the orientational order parameter, 


$$
S=\frac{1}{n}\left\langle\sum_{i=1}^{n} \sum_{j=0}^{N_{A}-1} \frac{3 \cos ^{2} \alpha_{j, j+1}-1}{2\left(N_{A}-1\right)}\right\rangle,
$$

where $\cos \alpha_{j, j+1}=\mathbf{n}(\mathbf{r}) \cdot\left(\mathbf{r}_{j+1}-\mathbf{r}_{j}\right) /\left\|\mathbf{r}_{j+1}-\mathbf{r}_{j}\right\|$ denotes the angle between the local normal vector, $\mathbf{n}(\mathbf{r})$, to the bilayer and the bond vector between successive neighboring hydrophobic beads $j$ and $j+1$. $i$ sums over all molecules in the bilayer and the average is taken over an ensemble of bilayers. $S=1$ indicates that the local director is perfectly aligned parallel to $\mathbf{n}(\mathbf{r}), S=0$ indicates that the director is isotropically distributed, and $S=-1$ indicates that the director is aligned in the plane of the bilayer. Since $\mathbf{n}(\mathbf{r})$ is a function of the coordinates of many lipids, its calculation involves a triangulation procedure [201], where we describe the bilayer midplane by a set of small triangles with a unique normal, $\mathbf{n}(\mathbf{r})$, in each triangle.

In the gel phase the lipids form a structure with sixfold orientational order. We probe this structure by the order parameter

$$
\psi_{6}=\frac{1}{n}\left\langle\left|\sum_{i=1}^{n} \frac{1}{n_{i}} \sum_{j=1}^{n_{i}} \exp \left(6 i \phi_{i j}\right)\right|\right\rangle .
$$

Here, $n_{i}$ denotes the number of lipids adjacent to lipid $i$, as determined by a Voronoi tesselation [214], and $\phi_{i j}$ the angle between the vector from the center of mass of lipid $i$ to that of lipid $j$, and some arbitrary but fixed direction in the plane of the bilayer. $\psi_{6}=1$ indicates perfect sixfold orientational order over the entire bilayer, whereas $\psi_{6}=0$ signals the absence of such order.

Both order parameters, $S$ and $\psi_{6}$, clearly distinguish between the $L_{\alpha}$ and the $L_{\beta}$ phase, but they differ in one crucial point: $S$ is composed of additive contributions, which only stem from conformational, single molecule properties and therefore its change in response to moving a segment can be easily computed. The opposite is true for $\psi_{6}$, which only reflects orientational order caused by intermolecular packing and requires the computationally intense Voronoi tessellation in order to identify the neighbors of a lipid. We choose $S$ as the single reaction coordinate for the main phase transition, because the conformational and the orientational transitions are coupled, and $S$ is considerably easier to compute than $\psi_{6}$.

\section{Free energy perturbation theory}

The free energy perturbation theory (FEP) is a formally exact way of calculating free energy differences between two systems with different Hamiltonians [213]. Here, FEP is used to calculate such differences between two bilayers whose non-bonded interactions differ in $\bar{\rho}^{*}$. To this end, we sample configurations at a reference density, $\bar{\rho}_{0}^{*}$, and calculate the free energy difference, 


$$
F\left(\bar{\rho}^{*}\right)-F\left(\bar{\rho}_{0}^{*}\right)=-k_{B} T \ln \left\langle e^{-\beta \Delta \mathcal{H}_{\mathrm{nb}}\left(\bar{\rho}^{*}\right)}\right\rangle_{0},
$$

to a system at density $\bar{\rho}^{*}$. Here $\langle\cdots\rangle_{0}$ stands for an ensemble average of the reference system and

$$
\begin{aligned}
\Delta \mathcal{H}_{\mathrm{nb}}\left(\bar{\rho}^{*}\right) & =\mathcal{H}_{\mathrm{nb}}\left(\bar{\rho}^{*}\right)-\mathcal{H}_{\mathrm{nb}}\left(\bar{\rho}_{0}^{*}\right) \\
& =\left[v_{\alpha \beta}\left(\bar{\rho}^{*}\right)-v_{\alpha \beta}\left(\bar{\rho}_{0}^{*}\right)\right] P_{\alpha \beta}+\left[w_{\alpha \beta \gamma}\left(\bar{\rho}^{*}\right)-w_{\alpha \beta \gamma}\left(\bar{\rho}_{0}^{*}\right)\right] Q_{\alpha \beta \gamma}
\end{aligned}
$$

is the difference of the non-bonded energy, cf. Eq. (2.14). $P_{\alpha \beta}$ and $Q_{\alpha \beta \gamma}$ are the integrated densities defined by

$$
\begin{aligned}
P_{\alpha \beta} & \equiv \frac{1}{2} \sum_{i} \delta_{\alpha \mathcal{T}(i)} \tilde{\rho}_{2 \beta}\left(\mathbf{r}_{i}\right) \\
Q_{\alpha \beta \gamma} & \equiv \frac{1}{3} \sum_{i} \delta_{\alpha \mathcal{T}(i)} \tilde{\rho}_{3 \beta}\left(\mathbf{r}_{i}\right) \tilde{\rho}_{3 \gamma}\left(\mathbf{r}_{i}\right) .
\end{aligned}
$$

Since FEP samples only the phase space of a single thermodynamic phase, i.e., the phase that is stable ${ }^{2}$ at $\bar{\rho}_{0}^{*}$, we cannot locate the coexistence of two phases. However, FEP is well suited to explore the free energy branch, $F\left(\bar{\rho}^{*}\right)$, of a single phase, i.e., relative changes in the free energy.

\section{Umbrella sampling}

In contrast to FEP, the combination of US and WHAM allows for a direct location of the phase coexistence [209-213]. This becomes possible by calculating the free energy profile, $F(S)$, as a function of the order parameter, $S(\mathbf{x})$, which is, in turn, a function of all particle coordinates $\mathbf{x}$. Let $S_{\mathrm{fl}}=0.212$ and $S_{\text {gel }}=0.625$ denote the values of the order parameter in the $L_{\alpha}$ and in the $L_{\beta}$ phase, respectively. To obtain $F(S)$, bilayer configurations in the interval $S_{\mathrm{fl}} \ldots S_{\text {gel }}$ have to be sampled. However, the unfavorable configurations in the miscibility gap are virtually unreachable by conventional Boltzmann sampling because their statistical weight is exponentially small. By including an additional US potential, $W_{i}$, we force the system to sample also these unfavorable configurations. Specifically, we add the harmonic potential

$$
W_{i}(\mathbf{x})=\frac{k}{2} n\left(N_{A}-1\right)\left[S(\mathbf{x})-S_{i}\right]^{2} .
$$

that biases the simulation to keep $S(\mathbf{x})$ in the vicinity of $S_{i}$. Here $k=1 k_{B} T$ is a spring constant that measures how strong deviations from $S_{i}$ are penalized; the prefactor makes $W_{i}(\mathbf{x})$ extensive. We have used an equidistant spacing of

\footnotetext{
${ }^{2}$ or at least metastable
} 
the $S_{i}$ in the range $0.15<S_{i}<0.8$ with $\Delta S_{i}=0.01$ to sample the whole interval uniformly.

In addition to $S_{i}$, also the expansion coefficients, which determine the nonbonded interactions, are varied. In each simulation with $W_{i}(\mathbf{x})$ and coefficients $v_{\alpha \beta}^{j}$ and $w_{\alpha \beta \gamma}^{j}$, a trajectory with $n_{i j}$ samples of the order parameter $S$, the total energy, $U$, and the integrated densities, $P_{\alpha \beta}$ and $Q_{\alpha \beta \gamma}$, is recorded. This trajectory is reweighted to study the thermodynamic behavior of the bilayer at other interaction parameters without performing additional simulations.

Each trajectory, $S_{i j, p}$, where $p=1 \ldots n_{i j}$ denotes the index of one specific sample, is binned into a normalized histogram $p_{i j}^{(b)}(S)$, that measures the biased probability density of visiting $S$. The corresponding unbiased probability density is defined as

$$
p_{i j}^{(u)}(S)=e^{\beta\left[W_{i j}(S)-f_{i j}\right]} p_{i j}^{(b)}(S)
$$

where the $f_{i j}$ are the (yet unknown) free energy differences arising from

$$
W_{i j}(\mathbf{x})=\Delta \mathcal{H}_{\mathrm{nb}}\left(\mathbf{x}, \bar{\rho}_{j}\right)+W_{i}(\mathbf{x}),
$$

with respect to some arbitrary reference state, which is in our case $\bar{\rho}_{0}^{*}=17$.

The idea of WHAM is to combine all unbiased histograms $p_{i j}^{(u)}(S)$ into a single optimal histogram $p_{0}(S)$, which is given by a weighted superposition of the $p_{i j}^{(u)}(S)$, so that the statistical error is minimal [211, 212]. First, the free energies, $f_{i j}$, arising from the biasing potential are calculated from

$$
e^{-\beta f_{i j}}=\int d \mathbf{x} e^{-\beta W_{i j}(\mathbf{x})} p_{0}(S(\mathbf{x}))
$$

where the optimal probability density

$$
p_{0}(\mathbf{x})=C \sum_{i j} \sum_{p=1}^{n_{i j}} \frac{\delta\left(\mathbf{x}-\mathbf{x}_{i j, p}\right)}{\sum_{k l} e^{-\beta\left[W_{k l}\left(\mathbf{x}_{i j, p}\right)-f_{k l}\right]}}
$$

is formally available, once the free energies $f_{i j}$ have been computed, i.e., a nonlinear set of coupled equations has been solved. Here $C$ is a normalization constant, which is chosen, such that the first run has the free energy $f=$ 0 . Once the $f_{i j}$ are available, the free energy, $F\left(\bar{\rho}^{*}\right)$, can be computed by reweighting via

$$
e^{-\beta F\left(\bar{\rho}^{*}\right)}=\int d \mathbf{x} e^{-\beta \mathcal{H}_{\mathrm{nb}}\left(\mathbf{x}, \bar{\rho}^{*}\right)} p_{0}(\mathbf{x})
$$

In contrast to FEP, Eq. (3.23), $F\left(\bar{\rho}^{*}\right)$ includes this time the contributions from both phases. 
The probability density, $p\left(\mathcal{O}, \bar{\rho}^{*}\right)$, of any observable, $\mathcal{O}(\mathbf{x})$, as a function of $\bar{\rho}^{*}$ is formally available by

$$
p\left(\mathcal{O}, \bar{\rho}^{*}\right)=\int d \mathbf{x} p\left(\mathbf{x}, \bar{\rho}^{*}\right) \delta(\mathcal{O}-\mathcal{O}(\mathbf{x})),
$$

where the full probability density, $p\left(\mathbf{x}, \bar{\rho}^{*}\right)$, is given by

$$
p\left(\mathbf{x}, \bar{\rho}^{*}\right)=p_{0}(\mathbf{x}) e^{-\beta\left[\mathcal{H}_{\mathrm{nb}}\left(\mathbf{x}, \bar{\rho}^{*}\right)-F\left(\bar{\rho}^{*}\right)\right]} .
$$

Equation (3.33) can be used to calculate the free energy of any observable as a function of $\bar{\rho}^{*}$ by taking the negative logarithm, i.e.,

$$
F\left(\mathcal{O}, \bar{\rho}^{*}\right)=-k_{B} T \ln p\left(\mathcal{O}, \bar{\rho}^{*}\right) .
$$

During the calculations of the $f_{i j}$ one frequently encounters expressions like $e^{-\beta E}$, where $|\beta E|>500$ ( $E$ is extensive). Computations with standard double precision fail because of over- or underflows. We have circumvented this issue by usage of a special floating point library with a much greater precision [215]. However, this library requires huge amounts of computing time and memory so that a parallel code has been written in which the recurring loops $\sum_{i j} \sum_{p} \ldots$ in Eq. (3.31) have been distributed over 64-1024 CPUs. We have used a variant of Powell's hybrid solver method to solve for the $f_{i j}$ in Eq. (3.30).

\subsubsection{Location of the transition point}

\section{Hysteresis loop}

A first estimate of the position of the main phase transition is obtained from the center and the width of a hysteresis loop. Therefore we have simulated preassembled bilayers in the $N P_{t} T$-ensemble with $n=1600$ that were initially in the $L_{\alpha}$ phase at $\bar{\rho}^{*}=40$. We have performed several sequential cycles with $\bar{\rho}^{*}$ running from 11 to 40 and vice versa in steps of $\Delta \bar{\rho}^{*}=0.5$ or 1.0 for $\kappa N=50,75,100,125$. At each step the bilayer is simulated for $\Delta t=100 \tau$.

Near the main phase transition large hysteresis effects occur in $S$ and $\psi_{6}$ as shown in Fig. 3.9. The loops for both order parameters differ only quantitatively: they are weakly shifted and conformational order, measured by $S$, persists up to slightly higher values of $\bar{\rho}^{*}$ than the orientational order, measured by $\psi_{6}$. Their widths grow with increasing $\kappa N$, i.e., metastable domains persist up to higher $\bar{\rho}^{*}$. The increasing amplitudes of the order parameters indicate that different thermodynamic phases show up. For instance, at $\kappa N=125$ the $L_{\beta}$ and the $L_{\beta^{\prime}}$ phases occur and their transition is visible as a dip in both order parameters near $\bar{\rho}^{*} \approx 20$. For $\kappa N<100$ hardly any hysteresis effects are visible. 


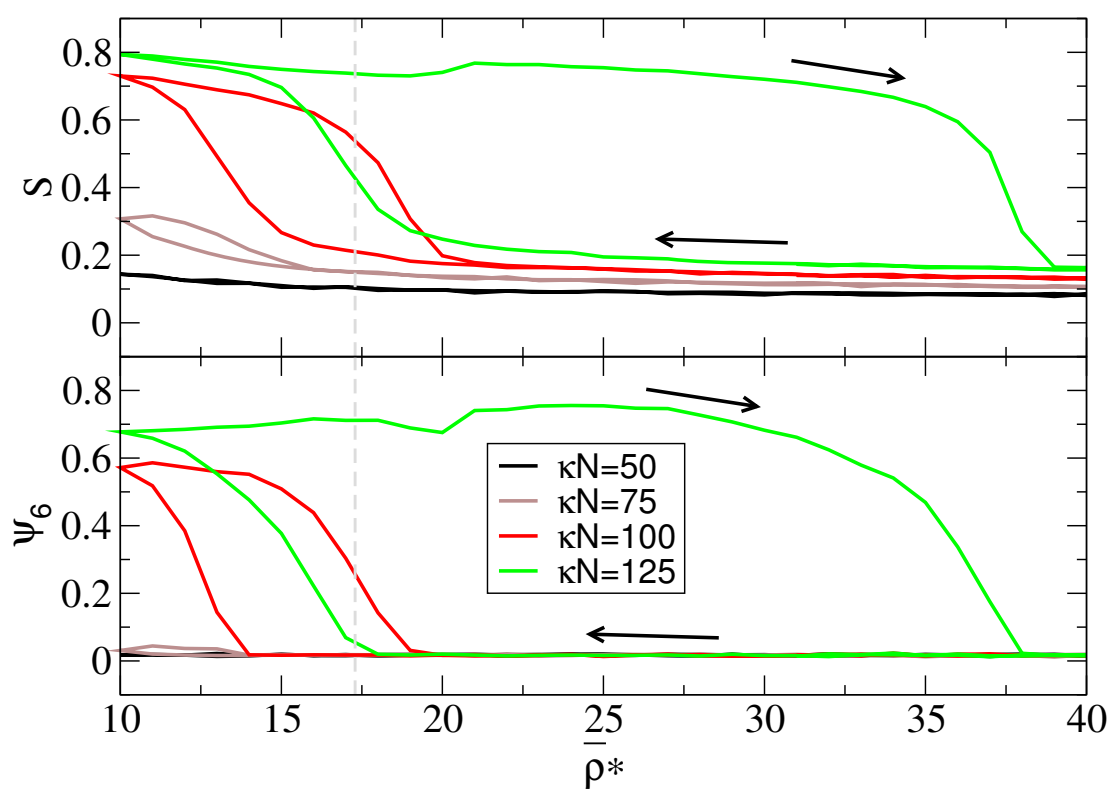

Figure 3.9: Hysteresis loops of $S$ (top) and $\psi_{6}$ (bottom) for $\kappa N=50 \ldots 125$. The arrows mark the direction in which $\bar{\rho}^{*}$ is proceeded. The dashed line at $\bar{\rho}^{*}=17.27$ indicates the transition point for $\kappa N=100$ which is determined below. Reprinted with permission from Ref. 178. Copyright 2010, American Institute of Physics.

We focus on the system $\kappa N=100$ where the phase diagram, Fig. 3.2, suggests in agreement with the hysteresis loop, Fig. 3.9, that only two phases, $L_{\alpha}$ and $L_{\beta}$, exist. The main phase transition is located in the interval $15<\bar{\rho}^{*}<19$. Hence, our first estimate for the point of the phase transition is the center of this interval, i.e.,

$$
\bar{\rho}_{\mathrm{HYST}}=17(2) .
$$

\section{Reweighting}

We calculate $F(S)$ by means of US/WHAM from simulations at different densities, $\bar{\rho}^{*}=16.28,17.00$, and 17.29 , with 2-5 different initial configurations in the $\mathrm{NP}_{t} \mathrm{~T}$ ensemble. Each biasing potential $W_{i}$, Eq. (3.27), is simulated for $\Delta t=3000 \tau$. It is advantageous to start the simulation from an initial configuration where both phases are already present.

The inset of Fig. 3.10 shows $F(S)$ at $\bar{\rho}^{*}=17$. The two visible minima correspond to the $L_{\alpha}$ and the $L_{\beta}$ phase. The offset, $\Delta F=648 k_{B} T$, between these minima indicates that the $L_{\beta}$ phase is stable and the $L_{\alpha}$ phase is metastable. To locate the phase transition, we reweight in $\bar{\rho}^{*}$ searching for a rapid variation, i.e., a rounded discontinuity, of the slope that signals the phase transition. 


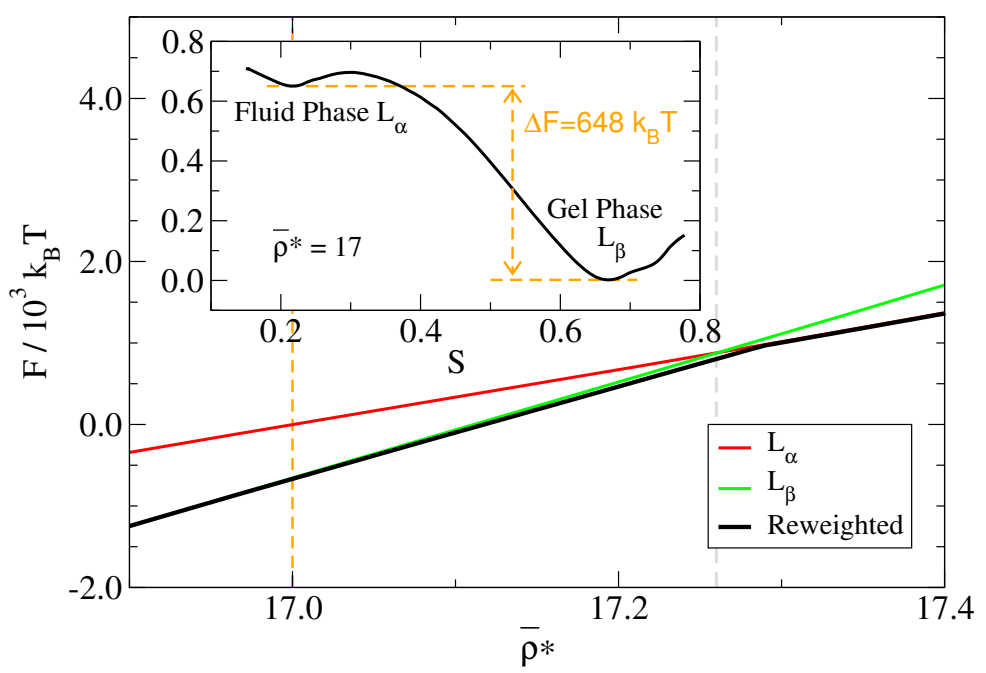

Figure 3.10: Inset: Free energy, $F(S)$, at $\bar{\rho}^{*}=17$. There is a difference of $\Delta F=$ $648 k_{B} T$ between the minima of the $L_{\alpha}$ phase and the $L_{\beta}$ phase. Main Panel: $F\left(\bar{\rho}^{*}\right)$ obtained from histogram reweighting in comparison to two independent FEP calculations of a $L_{\alpha}$ and a $L_{\beta}$ phase, whose offset, $\Delta F$, at $\bar{\rho}^{*}=17$ is known from the inset. Both curves intersect at $\bar{\rho}_{\mathrm{FEP}}=17.26(5)$ (dashed gray line). Reprinted with permission from Ref. 178. Copyright 2010, American Institute of Physics.

Such a variation occurs at

$$
\bar{\rho}_{\mathrm{US}}=17.29(1)
$$

and indicates the crossing of the free energy branches of the different phases (solid black line in the main panel of Fig. 3.10).

It is convenient to introduce a normalized order parameter,

$$
\Delta S \equiv \frac{S-S_{\mathrm{fl}}}{S_{\mathrm{gel}}-S_{\mathrm{fl}}}
$$

so that the minima of the free energy in the fluid phase at $S_{\mathrm{fl}}$ and in the gel phase at $S_{\text {gel }}$ correspond to $\Delta S=0$ and $\Delta S=1$, respectively.

At $\bar{\rho}^{*}=17.29$ both phases have equal statistical weight and are separated by a free energy barrier of $151.0(5) k_{B} T$ (see Fig. 3.11, left). Gratifyingly the US/WHAM results for the free energy, $F(\Delta S)$ and $F\left(\bar{\rho}^{*}\right)$, are consistent which indicates the high statistical accuracy of our data.

It is interesting to note that the free energy profile, Fig. 3.11 (left), is asymmetric around $\Delta S=0.5$ and slightly tilted to the left. This asymmetry stems from the onset of orientational order. For $\Delta S<0.5$ only a small amount of the lipids has a straight conformation and it is unfavorable to form gel domains. Hence, no sixfold orientational order, measured by $\psi_{6}$, is visible (right panel 


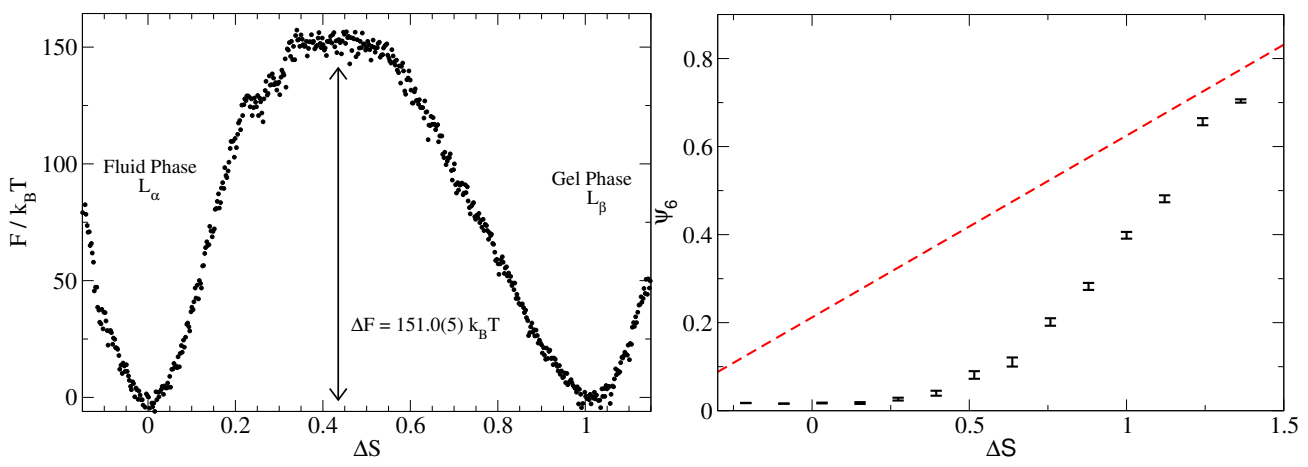

Figure 3.11: The free energy profile, $F(\Delta S)$, at phase coexistence determined by US/WHAM (left). The asymmetry around $\Delta S=0.5$ is related to the nonlinear coupling between $\Delta S$ and $\psi_{6}$ (right). The dashed line denotes a fictive linear coupling of $S$ and $\psi_{6}$. Reprinted with permission from Ref. 178. Copyright 2010, American Institute of Physics.

of Fig. 3.11). For $\Delta S>0.5$ enough lipids acquired a straight conformation so that gel domains form and an orientational order sets in. This leads to an accelerated decrease of $F(\Delta S)$. The minimal free energy is finally reached in a state with a global sixfold orientational order. Only the trailing tail beads of the lipids interdigitate with the ones from the opposite leaflet and constitute a thin, disordered layer at the center of the bilayer.

\section{Free energy perturbation theory}

We have conducted two additional, independent simulations at $\bar{\rho}^{*}=17$ in the $N P_{t} T$ ensemble without an US potential. One initial configuration was prepared in the metastable $L_{\alpha}$ phase and the other was prepared in the $L_{\beta}$ phase. The free energy branches of each phase are extrapolated with FEP, cf. Eq. (3.23). In this method the relative free energy difference between both branches remains undetermined. However, the offset at $\bar{\rho}^{*}=17, \Delta F=$ $648 k_{B} T$, has already been computed with US/WHAM, cf. Fig. 3.10. The main panel of this figure depicts the two, correspondingly shifted branches of $F\left(\bar{\rho}^{*}\right)$, which intersect at

$$
\bar{\rho}_{\mathrm{FEP}}=17.26(5) .
$$

In Fig. 3.10 we present the two branches of $F\left(\bar{\rho}^{*}\right)$ obtained from FEP in comparison to the result from US/WHAM. In the $L_{\alpha}$ phase both methods completely agree, however, in the $L_{\beta}$ phase there is a small difference discernible. Between $\bar{\rho}^{*}=17.15$ and 17.29 the FEP calculation slightly overestimates the free energy per lipid by $\Delta F / n=0.02 k_{B} T$, which arises from a gradual loss of sixfold orientational order as the transition is approached from the $L_{\beta}$ phase. 


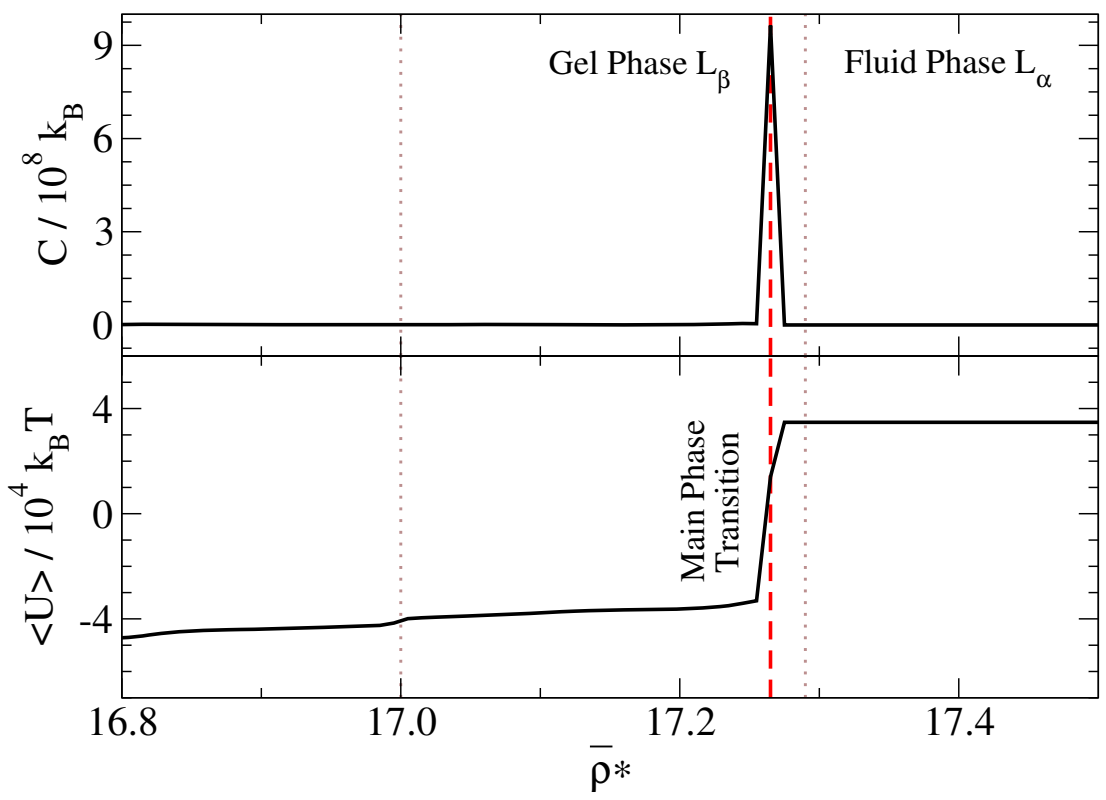

Figure 3.12: Specific heat, $C$, (top) and total energy, $\langle U\rangle$, (bottom) obtained from histogram reweighting as a function of $\bar{\rho}^{*}$. The main phase transition is visible as a peak in $C$ at $\bar{\rho}_{\mathrm{SH}}=17.27$ (dashed red line) and a sharp rise in $\langle U\rangle$. The dotted gray lines mark the points where US simulations were done. Reprinted with permission from Ref. 178. Copyright 2010, American Institute of Physics.

Therefore the result, $\bar{\rho}_{\mathrm{FEP}}$, is less accurate.

A similar way of determining the phase coexistence point has been applied earlier. In Ref. 108 the relationship between the branches is fixed by the knowledge of the two bulk free energies, which were extracted from mean-field theory. A similar calculation is possible in the fluid phase of our coarse-grained model, but it is not accurate in the gel phase, where correlations between the lipids are essential. These correlations, which are captured by our simulations, are clearly visible, e.g., in the order parameter $\psi_{6}$.

\section{Specific heat}

In the gel phase, the conformational degrees of freedom of the molecules and the translational degrees of freedom of the centers of mass are frozen and do not contribute to the total energy $\langle U\rangle$. These degrees of freedom are unlocked in the fluid phase and much larger values of $\langle U\rangle$ occur. The expected jump in the total energy or the large variation, $\left\langle U^{2}\right\rangle-\langle U\rangle^{2}$, which is proportional to the specific heat, $C\left(\bar{\rho}^{*}\right)$, can be used as another means to determine the transition point. 
We employ the reweighting procedure, Eq. (3.33), once more to calculate the probability distribution, $p\left(\bar{\rho}^{*}, U\right)$, and from this $\langle U\rangle\left(\bar{\rho}^{*}\right)$ as well as

$$
C\left(\bar{\rho}^{*}\right)=k_{B} \frac{\left\langle U^{2}\right\rangle\left(\bar{\rho}^{*}\right)-\langle U\rangle^{2}\left(\bar{\rho}^{*}\right)}{\left(k_{B} T\right)^{2}} .
$$

As expected, $p\left(\bar{\rho}^{*}, U\right)$ has for $\bar{\rho}^{*}<17.2$ a single peak at negative energies, $U_{\text {gel }} \approx-4 \times 10^{4} k_{B} T$, and for $\bar{\rho}^{*}>17.3$ a single peak at positive energies, $U_{\text {fluid }} \approx+4 \times 10^{4} k_{B} T$. In the phase transition region, $p\left(\bar{\rho}^{*}, U\right)$ has a bimodal shape with two peaks at $\pm 4 \times 10^{4} k_{B} T$, thus, the variance of this distribution at fixed $\bar{\rho}^{*}$ acquires a sharp peak (see Fig. 3.12, top). We obtain another estimate of the main phase transition from the location of this peak,

$$
\bar{\rho}_{\mathrm{SH}}=17.27(2) .
$$

The difference between these two energies, $\Delta U=U_{\text {fluid }}-U_{\text {gel }} \approx 8 \times 10^{4} k_{B} T$, is the latent heat of the phase transition and gives rise to a sudden jump in $\langle U\rangle\left(\bar{\rho}^{*}\right)$ at the same position (cf. Fig. 3.12, bottom). The slow increase of $\langle U\rangle\left(\bar{\rho}^{*}\right)$ in the interval $16.8<\bar{\rho}^{*}<17.27$ can be related to a gradual melting of the hydrophobic tails, i.e., the conformational degrees of freedom start to get unlocked even before the main phase transition takes place. Since there is no additional peak visible in $C\left(\bar{\rho}^{*}\right)$ at smaller $\bar{\rho}^{*}$, this gradual melting is no pretransition [204].

\subsubsection{Discussion and conclusion}

Depending on the parameters $\kappa N$ and $\bar{\rho}^{*}$, the lipids self-assemble into bilayers of different thermodynamic phases. The main phase transition, $L_{\beta} \leftrightarrow L_{\alpha}$, has many of the characteristics well known from discontinuous phase transitions, like pronounced hysteresis effects, the occurrence of metastable states, and sharp peaks in the response functions.

In this section, we discussed the main phase transition in detail, computed its free energy profile as a function of the conformational order parameter, $S$, and provided four different (but not independent) methods for locating the transition point. The first crude estimate, $\bar{\rho}_{\mathrm{HYST}}=17(2)$, was obtained from the center and the width of the hysteresis loop. A more precise estimate, $\bar{\rho}_{\mathrm{US}}=17.29(1)$, is obtained from the combination of umbrella sampling and the weighted histogram analysis method. We used the free energy perturbation theory to extrapolate the branches of the two phases and to find their intersection point, thereby yielding $\bar{\rho}_{\mathrm{FEP}}=17.26(5)$. A fourth estimate was obtained by inspecting the specific heat at the phase transition, which has a sharp peak at $\bar{\rho}_{\mathrm{SH}}=17.27(2)$. 
The three latter estimates, $\bar{\rho}_{\mathrm{US}}, \bar{\rho}_{\mathrm{FEP}}$, and $\bar{\rho}_{\mathrm{SH}}$, nicely agree with each other. The main uncertainty of our estimate of the phase coexistence, however, stems from a possible insufficient sampling along the path that reversibly connects the liquid and the gel phase. Unfortunately, this uncertainty is difficult to quantify. The consistency of the results suggests that the main phase transition for $\kappa N=100$ occurs at

$$
\bar{\rho}_{\text {coex }}=17.3(1) .
$$

Thus, the accuracy in the location of the transition point is improved by a factor of 20 compared to the accuracy obtained from the hysteresis loop. Thus, with the combination of umbrella sampling and histogram reweighting it is now -at least in principle- possible to study the entire phase diagram and accurately determine all phase boundaries.

The free energy profile across the phase transition, $F(S)$, is asymmetric in the scalar order parameter, $S$, because of the complex nature of the main phase transition, where two different sets of degrees of freedom, the conformational and the translational, are coupled nonlinearly.

\subsection{Line tension and bilayers in the miscibility gap}

The lateral demixing of multi-component lipid membranes into domains with different compositions, "rafts", is an exciting phenomenon that is studied actively in experiments [17, 216-220], computer simulations [221, 222], and analytical calculations. It is believed that this lateral heterogeneity plays an important role in biomembranes and cells use it actively to control the properties of their membranes by switching the molecular composition [223, 224]. The line tension, which is the free energy per unit length of the domain boundaries, plays a crucial role in these studies and several different experimental methods for obtaining such line tensions have been proposed which all give values around $10 \mathrm{pN}[17,217-220,225]$.

The simplest system that shows such a lateral heterogeneity is, however, a single-component lipid membrane at coexistence of two thermodynamic phases. Therefore, we continue the discussion of the main phase transition by looking at the line tension between domains belonging to different thermodynamic phases and at the shape of these domains, which is closely related. The occurrence of droplet and slab shapes is a universal aspect of discontinuous phase transitions in finite systems [226-231]. We show how typical configurations of the finite bilayer inside the miscibility gap look like and how this is related to the free energy profile.

It seems to be widely unknown that there are two distinct definitions of the line tension in a two-dimensional system which should not be confused. In this work they are denoted as the bare line tension, $\lambda$, and the thermodynamic line 
tension, $\sigma$. The former one occurs in the power spectrum of line fluctuations, the latter one in measurements of free energies or interface lengths. We present a careful definition based on statistical mechanics, measure them in our model, and demonstrate their difference.

\subsubsection{Theory}

\section{Configurations in the miscibility gap}

Let us consider a lipid bilayer in the $L_{\alpha}$ phase at phase coexistence with the $L_{\beta}$ phase. If the normalized order parameter, $\Delta S$, is slightly increased from $\Delta S=0$, this small increment is distributed homogeneously throughout the bilayer. The excess free energy of this undercooled fluid bilayer up to second order in $\Delta S$ is given by a Taylor expansion around the minimum

$$
F_{\mathrm{uf}}=\frac{k_{\mathrm{uf}}}{2}(\Delta S)^{2},
$$

where $k_{\mathrm{uf}}$ is a constant measuring the response of the system to changes in $\Delta S$. The same is true for the other minimum where the free energy of an overheated gel phase is

$$
F_{\mathrm{og}}=\frac{k_{\mathrm{og}}}{2}(1-\Delta S)^{2}
$$

In a macroscopic system an undercooled bilayer is metastable and the lipids condense into two-dimensional droplets of radius $R$ that consist of the thermodynamically stable $L_{\beta}$ phase. In the framework of classical nucleation theory, the excess free energy of such a droplet,

$$
F_{\text {drop }}=2 \pi \sigma R
$$

is given by the droplet's perimeter and the thermodynamic line tension, $\sigma$. There is no bulk contribution to Eq. (3.45) from the interior of the droplet, because the $L_{\alpha}$ and the $L_{\beta}$ phase have the same free energy at coexistence. Since the lipids occupy in both phases roughly the same area, cf. Tab. 3.2, $\Delta S$ approximately quantifies the fractional area of the $L_{\beta}$ phase. Thus, the area of the droplet, $\pi R^{2} \simeq L^{2} \Delta S$, is in good approximation proportional to $\Delta S$. Combining with Eq. (3.45) we obtain $R \sim(\Delta S)^{1 / 2}$ and

$$
F_{\text {drop }}=2 \sigma L \sqrt{\pi \Delta S}
$$

If the two-dimensional droplet grows larger, its size becomes comparable to the linear dimension of the simulation box, $L$. Then it is more favorable to form a slab of the $L_{\beta}$ phase separated from the $L_{\alpha}$ phase by two plane interfaces of length $L$. In this case, $F(\Delta S)$ reaches a plateau at the center of the free energy 
profile where its value is independent of $\Delta S$, i.e.,

$$
F_{\text {slab }}=2 \sigma L
$$

The excess free energy of the slab is dominated by the interfacial free energy provided that the system is large enough for the two interfaces not to interact. $\sigma$ is readily obtained from the plateau value [226].

Increasing $\Delta S$ further, one observes the reverse set of configurations. The slab of the $L_{\alpha}$ phase grows thinner and thinner and at some point it becomes favorable to form a fluid droplet surrounded by the $L_{\beta}$ phase. The radius of the droplet decreases while $\Delta S$ increases. Finally, the droplet vanishes and the lipids form a homogeneous $L_{\beta}$ phase.

\section{Interface line fluctuations}

If the boundary line separating the two domains in the slab geometry is smooth and free of overhangs, we can describe its position by a function $h(x)$. The statistical properties of $h(x)$ are dictated by the capillary wave Hamiltonian [200, 232],

$$
\mathcal{H}_{\text {cap }}=\lambda \int_{0}^{L} \mathrm{~d} x \sqrt{1+\left[h^{\prime}(x)\right]^{2}} \approx \lambda L+\frac{\lambda}{2} \int_{0}^{L} \mathrm{~d} x\left[h^{\prime}(x)\right]^{2},
$$

where $\lambda$ denotes the bare line tension. The coordinate system is chosen such that the mean position of the boundary vanishes, i.e., $\langle h(x)\rangle=0$. An expansion of $h(x)$ in a Fourier series with wave number $q=2 \pi n / L, n \in \mathbb{Z}$,

$$
h(x)=\sum_{q} h_{q} e^{i q x}
$$

shows that $\mathcal{H}_{\text {cap }}$ becomes diagonal in $q$-space and that the modes decouple:

$$
\mathcal{H}_{\text {cap }}=\lambda L+\mathcal{H}_{f} \quad \text { with } \quad \mathcal{H}_{f} \equiv \frac{\lambda L}{2} \sum_{q}\left|h_{q}\right|^{2} q^{2}
$$

The Hamiltonian $\mathcal{H}_{f}$ is the starting point for all further calculations. On the one hand, the fluctuation power spectrum,

$$
\left\langle\left|h_{q}\right|^{2}\right\rangle=\frac{k_{B} T}{\lambda L q^{2}}
$$

is readily obtained from Eq. (3.50) by using the equipartition theorem.

On the other hand, we can use $\mathcal{H}_{f}$ to calculate the free energy contribution, $\Delta F=F-\lambda L$, from the fluctuations of the boundary line. The canonical 
partition function, $\mathcal{Z}$, involves a functional integral over all $h(x)$, which is equivalent to an integral over all complex Fourier coefficients, $h_{q}$ :

$$
\mathcal{Z} \sim \int \mathcal{D}\left[h_{q}\right] \exp \left(-\frac{\mathcal{H}_{f}}{k_{B} T}\right) \sim \prod_{q} \int \frac{\mathrm{d} h_{q}}{L} \exp \left(-\frac{\lambda L}{2 k_{B} T}\left|h_{q}\right|^{2} q^{2}\right) .
$$

Since $h(x)$ is a real-valued function, $h_{q}$ has the Hermitian redundancy, i.e., $h_{-q}=h_{q}^{*}$. A decomposition into separate integrals over the real and the imaginary parts of $h_{q}$ for only the positive modes, $q>0$, thus yields

$$
\prod_{q} \int \frac{\mathrm{d} h_{q}}{L}=\prod_{q>0} \int \frac{\mathrm{d}\left(\operatorname{Re} h_{q}\right)}{L} \frac{d\left(\operatorname{Im} h_{q}\right)}{L} .
$$

We evaluate $\mathcal{Z}$ by carrying out the Gaussian quadratures and obtain

$$
\mathcal{Z} \sim \prod_{q>0} \frac{2 \pi k_{B} T}{\lambda L} \frac{1}{(q L)^{2}}
$$

To compute the free energy, $\Delta F=-k_{B} T \ln \mathcal{Z}$, the range of wave vectors has to be restricted. The simplest method is to introduce cutoffs for small as well as for high $q$. The cutoff at small $q$ arises from the periodic boundary conditions, i.e., $q_{\min }=2 \pi / L$. The UV cutoff, $q_{\max }=2 \pi n_{\max } / L=2 \pi / a, n_{\max }=L / a$, is delicate, because it introduces an additional length scale. This length scale, $a$, characterizes the smallest length on which the fluctuations of the boundary line can be described by the capillary wave Hamiltonian. Using these two cutoffs we obtain

$$
\begin{aligned}
\frac{\Delta F}{k_{B} T} & =-\ln \mathcal{Z}=\sum_{n=1}^{n_{\max }} \ln \left(\frac{\lambda L}{2 \pi k_{B} T} \cdot 4 \pi^{2} n^{2}\right) \\
& =n_{\max } \ln \left(\frac{2 \pi \lambda L}{k_{B} T}\right)+2 \ln \left(n_{\max } !\right) .
\end{aligned}
$$

$\Delta F$ is an extensive quantity proportional to $L$,

$$
\frac{\Delta F}{k_{B} T}=\frac{L}{a}\left[\ln \left(\frac{2 \pi \lambda L^{3}}{a^{2} k_{B} T}\right)-2\right] \equiv \frac{C L}{k_{B} T},
$$

and the total interfacial free energy is given by

$$
F=\lambda L+\Delta F=(\lambda+C) L \equiv \sigma L
$$

Thus, the bare line tension, $\lambda$, differs from the thermodynamic line tension, $\sigma$, by a term, $C$, which stems from the fluctuations of the boundary line and logarithmically depends on $L$ and $a$. 


\section{Determination of the interface line}

Several different schemes to locate the position of an interface are known [233]. Here we use an integral criterion [234, 235] in which we subdivide the profile of the local order parameter, $S(y, z)$, into $N_{z}=16$ horizontal stripes with a width of $\Delta z=L_{z} / N_{z}$. Each stripe is binned into two histograms, one for each leaflet, with a bin width of $\Delta y=2 r_{c}$, yielding in total $2 N_{z}$ histograms per snapshot. The bulk values of the order parameter in the gel phase, $S_{\text {gel }}$, and in the fluid phase, $S_{\mathrm{fl}}$, are extracted for every snapshot. As illustrated in Fig. 3.13, $h\left(z_{i}\right)$ in stripe $i$ has been calculated for each side of the slab separately as the position of the Gibbs dividing surface, such that

$$
\int_{h-\delta y}^{h} d y\left(S\left(y, z_{i}\right)-S_{\mathrm{fl}}\right)=\int_{h}^{h+\delta y} d y\left(S_{\mathrm{gel}}-S\left(y, z_{i}\right)\right) .
$$

Here $\delta y=10 r_{c}$ is used to confine the integral to a narrow region surrounding the Gibbs dividing surface so that fluctuations of the bulk influence the position of the interface minimally. Once $h(z)$ is determined, $\left|h_{q}\right|^{2}$ is available by an FFT. Similarly to the case of bilayer undulations, the average value, $\left\langle\left|h_{q}\right|^{2}\right\rangle$, is divided by a spectral damping factor $\left[\sin \left(\pi n / N_{z}\right) /\left(\pi n / N_{z}\right)\right]^{2}[181]$.

We compare two different ways of calculating this average. On the one hand, we argue that the bilayer is essentially a two-dimensional object, so that only the boundary lines at different sides of the slab, but not on different leaflets, fluctuate independently. This leads to an average calculated over two lines per snapshot. On the other hand, we can locate the boundaries in each leaflet independently, so that the average includes four lines per snapshot. In the limit $q \rightarrow 0$ we expect that the two boundaries in the two leaflets are coupled and that both methods yield the same $\lambda$.

\subsubsection{Results and discussion}

\section{Configurations in the miscibility gap}

First we studied the bilayer configurations in the miscibility gap. Figure 3.14 shows three such configurations: a gel droplet surrounded by the fluid phase, a slab geometry, and a fluid droplet surrounded by the gel phase. Additionally, it shows the corresponding Voronoi tesselations to illustrate the spatial distribution of the lipids.

A more quantitative analysis is available by a careful inspection of $F(\Delta S)$ at phase coexistence, cf. Fig. 3.15. The positions of the three configurations are marked by green circles. A fit to Eq. (3.43) or Eq. (3.44) in the vicinity of the two minima yields $k_{\mathrm{uf}}=6750 k_{B} T$ and $k_{\mathrm{og}}=4290 k_{B} T$; the resulting parabolas 


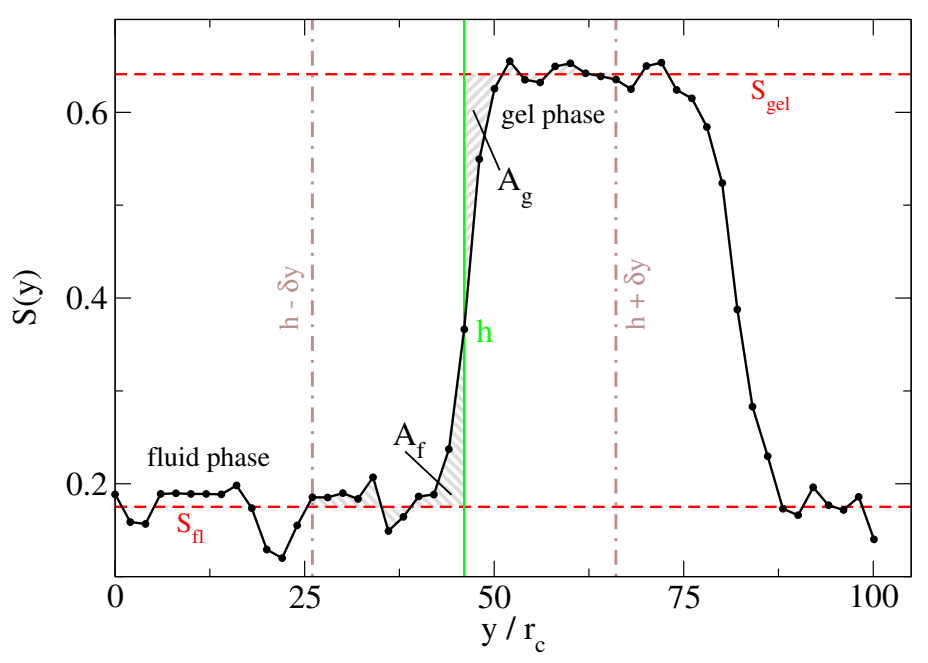

Figure 3.13: To calculate the Gibbs dividing surface the bulk values of the order parameter, $S_{\text {gel }}$ and $S_{\mathrm{fl}}$, are computed (dashed lines). After that, the integral criterion is applied in a narrow interval $h-\delta y \cdots h+\delta y$ surrounding each edge of the slab. The position of the interface $h$ is chosen so that $A_{f}=A_{g}$. Reprinted with permission from Ref. 178. Copyright 2010, American Institute of Physics.

are indicated in Fig. 3.15 by the blues curves. The agreement between the fits and the actual $F(\Delta S)$ is, particularly for the $L_{\alpha}$ phase, very good. Due to the aforementioned asymmetry of the free energy profile, cf. Sec. 3.2.2, the right edge of the free energy barrier has a different shape and the harmonic approximation for the minimum of the $L_{\beta}$ phase has a smaller range of validity.

The free energies of the gel and the fluid droplets, $F_{\text {drop }}$, cf. Eq. (3.46), are also shown in Fig. 3.15 as red, dash-dotted lines. They fit $F(\Delta S)$ very well at the left edge of the profile, but fail at the right edge where fluid droplets in the $L_{\beta}$ phase occur. Finally, we present the constant free energy of the slab geometry, cf. Eq. (3.47), $F_{\text {slab }}=151.0(5) k_{B} T$.

Additional deviations from the simple phenomenological estimates arise from the interaction between the lines that separate the liquid and the gel domains. For instance, the thermal fluctuations of the two lines in the slab geometry induce attractive Casimir forces [236]. While the slab is growing thinner and thinner at the crossover to the droplet geometry, these Casimir forces become more pronounced and reduce the free energy. However, there is an additional reduction of the free energy coming from transversal line fluctuations [237]. These become pronounced for small widths of the slab and finally lead to its destruction. While it is in principle possible to study these fluctuation mediated interactions by a careful inspection of $F(\Delta S)$, we did not investigate these effects in further detail. 

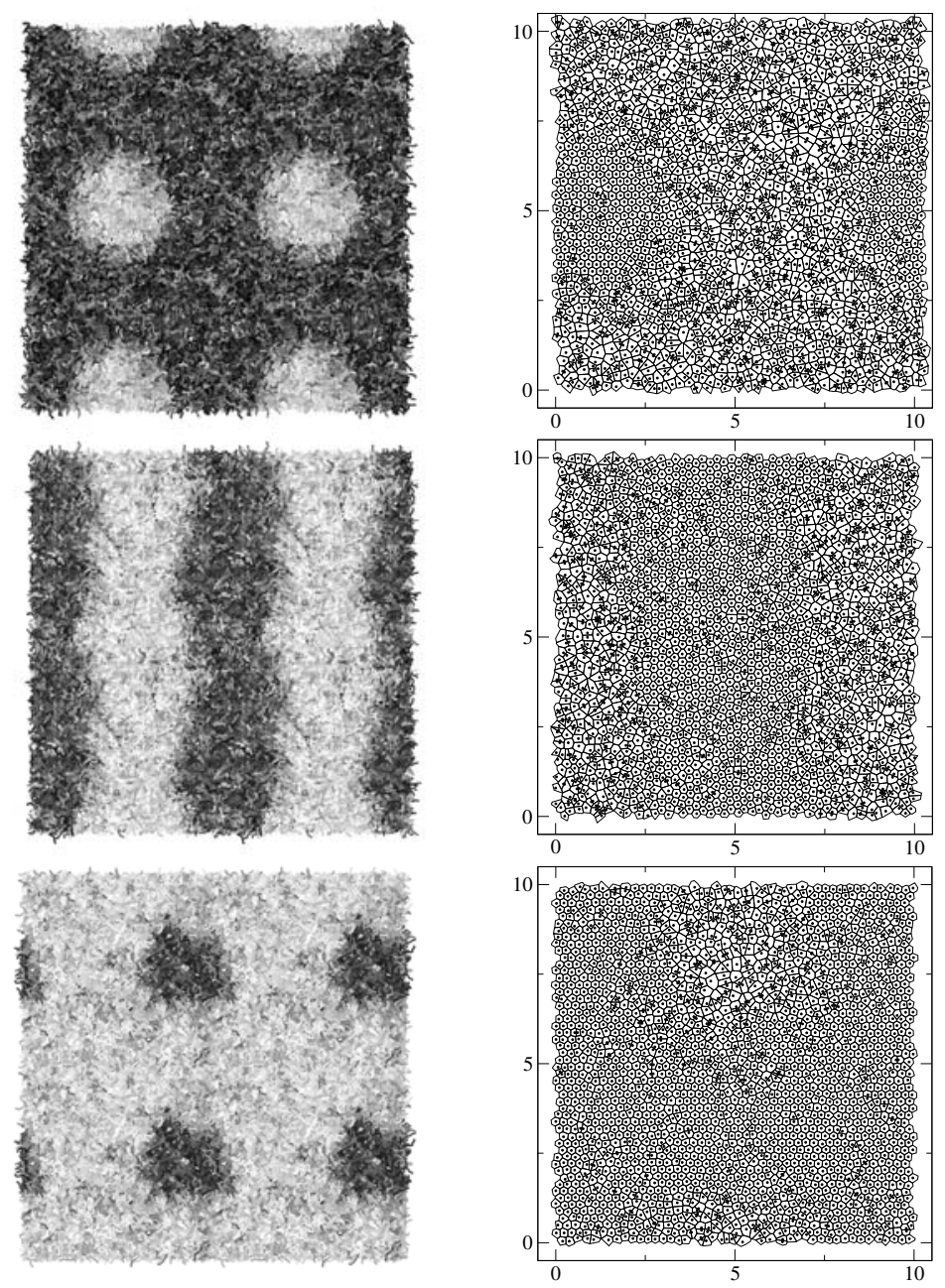

Figure 3.14: Bilayer configurations in the miscibility gap (duplicated across periodic boundaries). Left, from top to bottom: $L_{\beta}$ droplet in $L_{\alpha}$ phase $(\Delta S=0.26)$, the slab geometry $(\Delta S=0.60)$, and $L_{\alpha}$ droplet in a $L_{\beta}$ phase $(\Delta S=0.84)$. Each lipid is colored by its local conformational order parameter: the $L_{\beta}$ phase is white, the $L_{\alpha}$ phase dark, and intermediate values gray. Right: Voronoi tesselation of the same configurations as before, the dots are the molecular centers of mass. Reprinted with permission from Ref. 178. Copyright 2010, American Institute of Physics. 


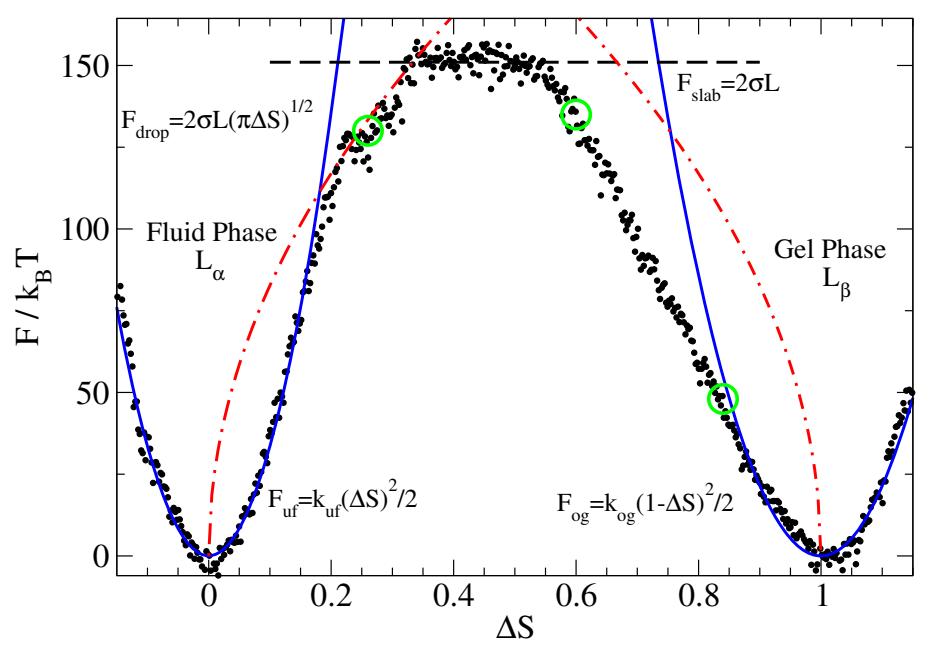

Figure 3.15: $F(\Delta S)$ at phase coexistence compared to the phenomenological expressions from Eq. (3.43), Eq. (3.46), and Eq. (3.47). Green circles mark the values of $\Delta S$ at which typical configurations are visualized in Fig. 3.14. Reprinted with permission from Ref. 178. Copyright 2010, American Institute of Physics.

\section{Bare line tension}

To record the fluctuation spectrum of the interface line, a rectangular shape of the simulation box, with $L_{y}>L_{z}$, was chosen. In this asymmetric situation the slab attains the lowest interfacial free energy if it aligns parallel to the $z$-axis. In this way the orientation of the interfaces is dictated by the box geometry. Specifically, we assembled an initial configuration with $n=14040$ lipids at $\bar{\rho}^{*}=17$ having $L_{y}=3 L_{z} \approx 105 r_{c}$. This configuration is simulated in the anisotropic $\mathrm{NP}_{t} \mathrm{~T}$ ensemble with two independent degrees of freedom. We employ an US potential with $S_{0}=0.34, k=5.0 k_{B} T$ for driving the system to the desired slab geometry. After an equilibration time of $\Delta t=10^{4} \tau$ the box lengths fluctuate around mean values of $\left\langle L_{y}\right\rangle=111.2 r_{c}$ and $\left\langle L_{z}\right\rangle=$ $35.0 r_{c}$, respectively, and both interfaces on both leaflets are flat. Unlike the experimental situation, where only domains of spherical shape are observed, there is no Laplace pressure because the interfaces are not curved and thus the two phases coexist at the same vanishing lateral pressure.

Figure 3.16 shows the line fluctuation spectra for the two different ways of averaging with two and four independent lines. In the inset, $\left\langle\left|h_{q}\right|^{2}\right\rangle q^{2}$ is plotted as a function of $(q L)^{2}$. For small $q$ this expression becomes linear and intercepts the $y$-axis at $k_{B} T / \lambda L_{z}$. The fits yield $\lambda_{2}=1.48 k_{B} T / r_{c}$ and $\lambda_{4}=1.24 k_{B} T / r_{c}$ for two and four lines, respectively.

For small $q$ both graphs in the fluctuation power spectrum of Fig. 3.16 asymptotically approach the same $q^{-2}$ power law. This indicates that interface fluc- 


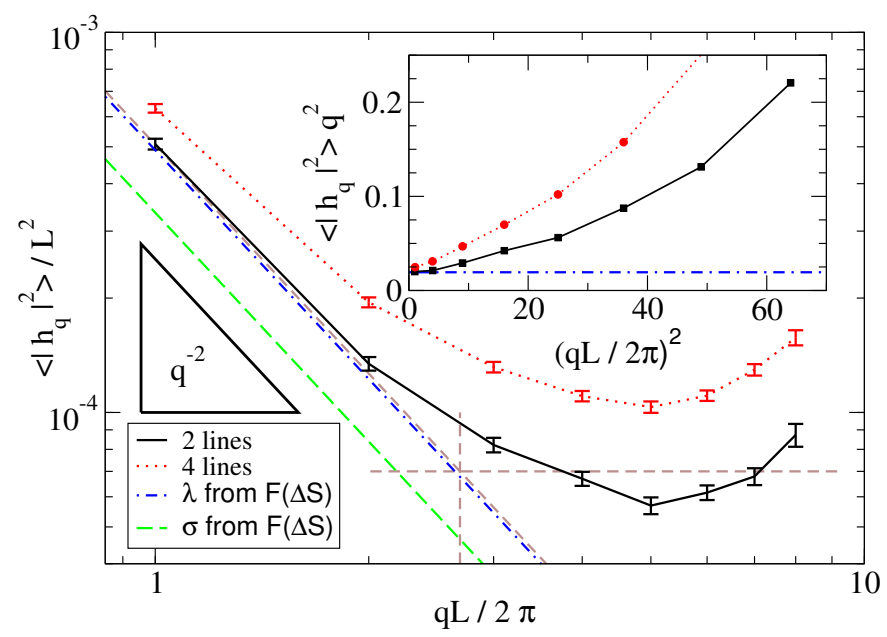

Figure 3.16: Power spectrum, $\left\langle\left|h_{q}\right|^{2}\right\rangle$, of the boundary line fluctuations calculated for 2 (solid) and 4 (dotted) independent interface lines with the standard mean error in comparison to the value calculated from $F(\Delta S)$ (dash-dotted). The dashed grey lines indicate the estimated value of the UV cutoff, $q_{\max }=2 \pi / a$. Inset: $\left\langle\left|h_{q}\right|^{2}\right\rangle \times q^{2}$ plotted as a function of $\left(q L_{z}\right)^{2}$. For small $q$ this function becomes constant and intersects the $y$-axis at $k_{B} T / \lambda L_{z}$. Reprinted with permission from Ref. 178. Copyright 2010, American Institute of Physics.

tuations are correlated on large scales. At larger values of $q$ the lines fluctuate independently and, concomitantly, the high- $q$ estimate of the line tension is lower for the data extracted from four lines than for two lines. The graph for $q L_{z} / 2 \pi>4$ shows clear deviations from the simple power law, which are expected because the description of the line by the capillary wave Hamiltonian breaks down on microscopic scales. Thus, $L_{z} \approx 35 r_{c}$ is too small to observe the expected scaling with $q^{-2}$ over an extended $q$-range. We compute the final result by taking the average of both values $\lambda_{2}$ and $\lambda_{4}$ and the average deviation as the uncertainty. This yields our final estimate of the bare line tension, $\lambda=1.36(12) k_{B} T / r_{c}$.

\section{Thermodynamic line tension}

By taking the plateau value, $F_{\text {slab }}=151.0(5) k_{B} T$, from Fig. 3.11 we obtain the thermodynamic line tension, $\sigma=2.16(1) k_{B} T / r_{c}$. This value can be converted to a bare line tension by solving Eq. (3.58) numerically for $\lambda$. We estimate the value of the UV cutoff, $a$, graphically from the intersect of the $q^{-2}$ power law at small $q$ and a constant fluctuation strength at higher $q$. We find $q_{\max }=$ $2 \pi / a=0.48 r_{c}^{-1}$, i.e., $a \approx 13 r_{c}$ and thus $n_{\max }=3$. This leads us to $\lambda_{F}=$ $1.55(1) k_{B} T / r_{c}$, which is in good agreement with the estimate obtained from the spectrum, $\lambda=1.36(12) k_{B} T / r_{c}$. 
To illustrate the difference between $\sigma$ and $\lambda_{F}$, we include in Fig. 3.16 the two asymptotical power spectra arising from $\lambda_{F}$ (dash-dotted line) and $\sigma$ (dashed line), when falsely inserted into Eq. (3.51). The former nicely describes the measured fluctuation spectrum, whereas the latter deviates significantly. Thus, there is a notable difference between $\sigma$ and $\lambda_{F}$ of approximately $30 \%$ even for the small system size considered in our simulation.

Using the previously determined conversion factor, Eq. (3.19), we get $5.2 \mathrm{pN}$ $=1 k_{B} T / r_{c}$ and find that our results, $\lambda=1.36(12) k_{B} T / r_{c}=7.1(6) \mathrm{pN}$ and $\lambda_{F}=1.55(1) k_{B} T / r_{c}=8.0(1) \mathrm{pN}$, show an excellent agreement with the experimental order of magnitude, $\lambda \approx 10 \mathrm{pN}$.

\subsubsection{Conclusions}

The lateral demixing of multi-component lipid membranes into domains with different compositions is an exciting phenomenon that is studied actively. The simplest system that shows such a lateral heterogeneity is a single-component lipid membrane at coexistence of two thermodynamic phases.

In this section we discussed the demixing behavior of lipids in the $L_{\alpha}$ and the $L_{\beta}$ phase at coexistence. We studied two aspects which naturally arise at discontinuous phase transitions: the occurrence of a line tension between fluid and gel domains, and the shape of these domains that arises as a consequence of the finiteness of our system.

The first part of this section dealt with the shape of the domains of the minority phase. We compared different regions in the free energy profile, $F(\Delta S)$, to phenomenological expressions. The first half of $F(\Delta S)$, where the fluid phase is the majority, follows the usual order of an undercooled, homogeneous phase, a droplet, and finally a slab of the minority phase; the phenomenological expressions fit $F(\Delta S)$ very well. The second half shows the reverse order, but the quantitative agreement of the expressions is slightly worse. This is attributed to the onset of orientational order, which is not captured by the scalar order parameter, $\triangle S$.

In the second part of this section, we studied the line tension between fluid and gel domains. We demonstrated that there are two different notions in a two-dimensional system, the bare and the thermodynamic line tension, which differ by a constant (here: $30 \%$ ) that depends on the length scale. This fact seems to be widely unknown in the literature - both line tensions are often used interchangeably. We obtained the bare line tension by recording the power spectrum of line fluctuations and the thermodynamic line tension from the plateau in $F(\Delta S)$. A conversion of the thermodynamic into a bare line tension yields a good agreement of both methods and we found $\lambda=1.55(1) k_{B} T / r_{c}=$ $8.0(1) \mathrm{pN}$. This agrees very well with the order of magnitude, $\lambda \approx 10 \mathrm{pN}$, that the experimentalists are observing for similar systems. 


\title{
4 Dynamic properties
}

\author{
"Biology was invented by God, physical \\ chemistry was invented by nice people, fluid \\ mechanics was invented by the devil."
}

(Harden McConnell)

One of the central ideas of coarse-graining is to integrate out microscopic degrees of freedom of an atomistic description so that an efficient model suitable for computer simulations is obtained. It has been shown in the previous chapter that our model reproduces many thermodynamical, structural, and mechanical properties of amphiphilic bilayers. However, the reduction of the degrees of freedom has an important side effect. They act as a heat bath for the mesoscopic degrees of freedom: they dissipate energy and create thermal noise. Since many degrees of freedom have been integrated out in the coarse-grained description, it is by no means clear that the coarse-grained dynamics still resembles the actual one. In fact, the study of how coarse-graining affects the dynamics is an active field that is emerging rapidly.

This chapter is devoted to the study of the dynamics of our model and is written with two slightly different aims in mind. On the one hand we measure many dynamic properties and compare them to their experimental counterparts. In this way we get an impression of the capabilities and shortcomings of the model. On the other hand we look for systematic deviations from atomistic simulations which are caused by the coarse-graining or the thermostat. We try to understand their origins and explain what could be done to mimic the atomistic behavior in a coarse-grained model.

There are three sections: in Sec. 4.1 we study the motion of the amphiphiles and try to shed some light on the recent discussion whether the amphiphiles move like Brownian particles on short times or if they exhibit collective motion. The next section, Sec. 4.2, focusses on the different mechanisms of dissipation. In general, three such mechanisms exist in bilayer membranes. These are the viscosity of the solvent, the surface viscosity of the bilayer, and the intermonolayer friction. The first one is missing due to the lack of solvent in our model, whereas the other two exist and are discussed in turn. Finally, we combine in Sec. 4.3 the results of the first two sections and create a two-dimensional representation of our model, which has the same lateral distribution functions and the same dissipation constants. In this simplified representation we investigate how the dynamics changes under the reduction of all intramolecular degrees of freedom. 


\subsection{Brownian vs. correlated motion}

In recent years there has been much debate about the question how lipids actually diffuse. The reason for this discussion is that different experimental techniques, which probe the lipids' lateral diffusion on different length scales, yield diffusion coefficients that differ by two orders of magnitude. On the one hand there are quasi-elastic neutron scattering experiments (QENS) that probe the extreme short-range diffusion on a length scale of two lipid diameters; they find apparent diffusion coefficients of $D \approx 10^{2} \mathrm{\mu m}^{2} / \mathrm{s}[238,239]$. On the other hand there are techniques like fluorescence recovery after photobleaching (FRAP), fluorescence correlation spectroscopy (FCS), and magnetic resonance experiments that probe the diffusion over several micrometers; they find $D \approx$ $1 \mathrm{\mu m}^{2} / \mathrm{s}$ [240-244].

Initially, this discrepancy was explained by the "free-volume theory" with a jump-diffusion model $[245,246]$. At short times a lipid is confined to a cage that is formed by the surrounding molecules. Its center of mass diffuses within the confinement and this "rattling in a cage" is seen by the QENS measurements as the fast diffusion. Occasionally a vacancy opens up within the surrounding molecules that is large enough so that the lipid can jump from its initial position into this vacancy. If the jump frequency is low enough one obtains a slow random walk with the diffusion coefficient seen by FRAP or FCS [247].

Although the free-volume theory is able to rationalize the experimental findings, its predictions for intermediate times could not be verified with the available experimental setups at that time. Indeed, they turned out to be wrong as soon as increased computing power became available and atomistic models of lipid bilayers were studied [248-251]. Only a tiny number of jump events were observed, and the long-range diffusive motion could not be explained by them alone. Instead, it turned out that the motion in the intermediate regime was much more involved and strongly collective [92, 252]. This led to the current picture in which the lipids dynamically assemble to clusters with a size of several nanometers and then diffuse collectively.

Since then, many simulation and experimental groups have been trying to shed some light on this collective motion. In a prominent work Falck et al. performed atomistic simulations of DPPC bilayers with 1152 and 4608 lipids for up to $100 \mathrm{~ns}$. They found no evidence for the free-volume theory, but instead they observed a flow-like pattern of the centers of mass of the lipids. They state that the "motion of lipids is correlated at least over tens of nanometers" [92]. Since the flow pattern exhibits some similarities with the string-like motion that is observed in glass forming materials [253, 254], other authors tried to understand this pattern by using mode-coupling theory [55], stretched exponential distributions [255], or other spatiotemporal displacement correlation 


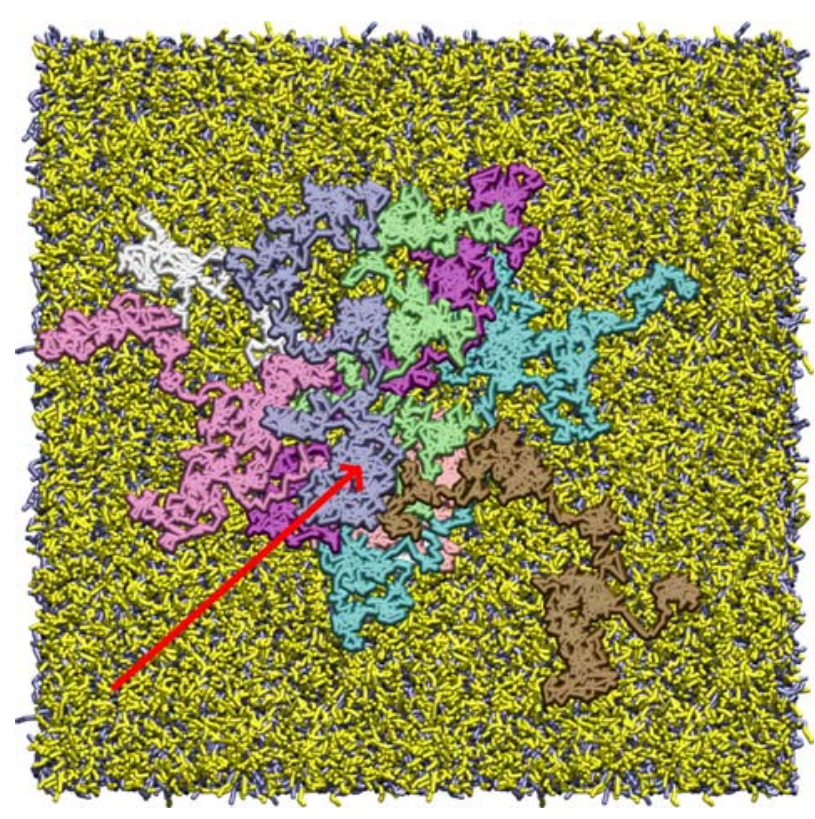

Figure 4.1: The picture shows a top view of a lipid bilayer (yellow, light blue) with the trajectories $(5000 \tau)$ of eight lipids that start next to each other in the area marked with the red arrow. Their long time motion resembles a random walk, but their intermediate motion is under active discussion.

functions [222, 256]. Currently, there is only one QENS experiment showing evidence of this flow motion [257].

The diffusion of lipids is not only restricted to the lateral direction, but they can also tunnel through the bilayer and come out on the other side. This diffusion in the normal direction, called "flipflop", is a thermally activated process and -due to the high energetic barrier- rare in synthetic membranes. ${ }^{1}$ A typical time between two flipflops is on the order of magnitude of $10^{4} \mathrm{~s}$ per lipid, i.e., it is much slower than any other process [258-260]. However, as a result of the much softer interactions too frequent flipflops are a common disease in many coarse-grained models [181, 261], so that this point deserves special attention.

Besides the study of Brownian motion many other things can be learnt from modern X-ray and neutron scattering experiments which yield the dynamic structure factor, $S(q, \omega)$ [262-265]. With these techniques it is possible to probe the lipid dynamics over several orders of magnitude in time and space. $S(q, \omega)$ is also available from simulations [57, 266, 267], so that a detailed comparison of the dynamics is possible. It is often interpreted in terms of the Rayleigh-Brillouin scheme for simple liquids where three lines occur [197]. The Rayleigh line at $\omega=0$ is related to the transport of heat and has a width that is proportional to the thermal diffusivity of the system. The two Brillouin lines at $\omega= \pm \omega_{B}(q)$ are related to the propagation of sound, i.e., $\omega_{B}(q)=c_{s} q$

\footnotetext{
${ }^{1}$ The situation in biomembranes is more complex, because special proteins, "flippases", exist which are able to catalyze flipflops.
} 
where $c_{s}$ is the adiabatic speed of sound. Their widths are proportional to the sound attenuation coefficient times $q^{2} . c_{s}$ and the attenuation coefficient have both been measured in experiments [263, 266] as well as in simulations [57], and typical values for $c_{s}$ are around $1400 \mathrm{~m} / \mathrm{s}$ (fluid phase) and $2300 \mathrm{~m} / \mathrm{s}$ (gel phase).

In this section we try to answer the question how the Brownian motion of the lipids in our model looks like and which role the collectivity plays in this process (see Fig. 4.1). In doing so we study many dynamic properties of individual lipids as well as their collective motion in the fluid and the gel phase. First, the diffusion coefficient of the lipids is calculated and used to establish a conversion factor which relates the unit of time in our model, $\tau$, to an experimental time scale. We also investigate the performance of our model with respect to the flipflops. Second, we calculate $S(q, \omega)$ to determine the dispersion relations and hence the spectrum of collective excitations of the bilayer. The speed of sound is extracted which serves as an upper boundary for all meaningful velocities. The thermal diffusivity cannot be obtained from our simulations, because the DPD thermostat flattens all temperature gradients. Since the Rayleigh line is difficult to observe even in simulations without such a thermostat, we omit its discussion in this work entirely. Finally, we unsuccessfully look for the flow pattern and explain its absence in our model.

\subsubsection{Methods}

\section{Mean-square displacement}

One of two different methods is normally employed for measuring a diffusion coefficient, $D$, in simulations. The idea of the first method, which goes back to Einstein [268], is to measure the mean-square displacement (MSD), $\left\langle\left(\mathbf{r}_{i}(t)-\right.\right.$ $\left.\left.\mathbf{r}_{i}(0)\right)^{2}\right\rangle$, of an ensemble of equal particles labelled by the index $i$ and then to look at the limit of long times. In two dimensions the MSD is

$$
\left\langle\left(\mathbf{r}_{i}(t)-\mathbf{r}_{i}(0)\right)^{2}\right\rangle=4 D t
$$

so that $D$ can be computed by dividing the left hand side of Eq. (4.1) by $4 t$ and by taking the limit $t \rightarrow \infty$.

However, the MSD is by itself an interesting function, because it visualizes the particle's motion on all time scales. Another analytically tractable limit is the one of very short times. Here, each particle ballistically moves a mean free path before a collision occurs. By using the equipartition theorem it can easily be shown that

$$
\left\langle\left(\mathbf{r}_{i}(t)-\mathbf{r}_{i}(0)\right)^{2}\right\rangle=2 k_{B} T t^{2} / m
$$

where $m$ is the mass of the considered particle. The functional form of these 
two limiting cases is universal, while the intermediate dynamics is non-universal and depends on the interactions.

In this work we look at the lateral diffusion of amphiphiles in a bilayer. Since the bilayer is soft, thermal undulations of the bilayer should be taken into account which lead to a diffusion on a curved surface. Hence, Eq. (4.1) is only an approximation for flat membranes. It has been shown by Reister and Seifert that it is still a good approximation as long as the system is not too large [269, 270]. Nevertheless, most particle-based simulation methods including our model- are currently unable to treat systems large enough for curvature effects to become important. In our case the error is smaller than $5 \%$ and can be safely ignored.

Normally, one would expect the distribution of MSDs at a fixed time $t$ to be a Gaussian. However, it is well known from many studies of glasses, that dynamic heterogeneities may exist, i.e., that there are certain mobile particles which move faster than other, immobile ones. If such a dynamic heterogeneity occurs, one sees a deviation from a Gaussian, specifically, one sees a flattening of the distribution, i.e., it becomes more platykurtic. Therefore, an easy way of detecting these dynamic heterogeneities is to look at the two-dimensional non-Gaussianity parameter (NGP) [271, 272],

$$
\alpha(t)=\frac{\left\langle\left(\mathbf{r}_{i}(t)-\mathbf{r}_{i}(0)\right)^{4}\right\rangle}{2\left\langle\left(\mathbf{r}_{i}(t)-\mathbf{r}_{i}(0)\right)^{2}\right\rangle^{2}}-1,
$$

which is zero for a Gaussian and positive for a platykurtic distribution.

The MSD as well as the NGP must be sampled over several orders of magnitude to bridge the ballistic and the diffusive limits. A calculation of these quantities at every instant of time is not only computationally demanding, but often unnecessary. In this work we use an "order- $n$ " algorithm that calculates them equidistantly on a logarithmic time scale and that automatically leads to an average over uncorrelated samples [273].

\section{Velocity autocorrelation}

The second method for calculating $D$ goes back to Green and Kubo (GK) who found a general method for computing friction and transport coefficients like diffusivities, heat conductivities, or viscosities. The idea is to measure the velocity autocorrelation function ( $\mathrm{VACF}),\left\langle\mathbf{v}_{i}(t) \cdot \mathbf{v}_{i}\right\rangle$, and to extrapolate carefully its time integral to infinity. It can be proven that it is equivalent to the Einstein method and that both produce the same results. ${ }^{2}$ Here, we use

\footnotetext{
${ }^{2}$ It seems that the Einstein method is generally preferred for computing $D$, because the time integration over the velocities is already done by the simulation so that the obtained results have a smaller statistical error.
} 
the relation [197]

$$
D=\frac{1}{2} \int_{0}^{\infty} d t\left\langle\mathbf{v}_{i}(t) \cdot \mathbf{v}_{i}\right\rangle .
$$

From a theoretical point of view, this quantity is ill-defined, because it can be shown analytically that $\left\langle\mathbf{v}_{i}(t) \cdot \mathbf{v}_{i}\right\rangle$ decays for long times like $t^{-d / 2}$, where $d$ is the dimensionality $[274,275]$. For $d=2$ this leads to a logarithmic divergence of $D$. However, a bilayer is not a strict two-dimensional object, so that it remains an open question with which exponent these long-time tails really decay. Even in our longest simulations, as well as in the experiments cited above, no divergence is observed, and we decided to ignore this issue.

Similar to the MSD, it is sufficient to calculate the VACF on a logarithmic time scale, thereby applying the order- $n$ algorithm once more.

\section{Intermediate scattering function}

Concerted motion is only detectable if one inspects dynamic correlations between several beads or molecules. The simplest approach is to look at correlations between two points $(\mathbf{r}, t)$ and $\left(\mathbf{r}^{\prime}, t^{\prime}\right)$ in space and time, but sometimes -for instance in the study of dynamic heterogeneities in glasses - it is also necessary to study higher order correlators [276, 277]. Here we restrict the discussion to two-point correlation functions, which simplifies the comparison with scattering experiments. Our starting point is the time-dependent local density of particles of type $\alpha$,

$$
\rho^{\alpha}(\mathbf{r}, t)=\sum_{i} \delta_{\mathcal{T}(i) \alpha} \delta\left(\mathbf{r}-\mathbf{r}_{i}(t)\right)
$$

where $\mathcal{T}(i)$ denotes the type of particle $i$, and $\delta_{\alpha \beta}$ is the usual Kronecker delta. Its spatial Fourier transform is given by

$$
\rho^{\alpha}(\mathbf{q}, t)=\sum_{i} \delta_{\mathcal{T}(i) \alpha} e^{-i \mathbf{q} \cdot \mathbf{r}_{i}(t)}
$$

where $\mathbf{q}$ is a wave vector commensurable with the periodic boundary conditions in the lateral direction. The autocorrelation function, $F^{\alpha}(\mathbf{q}, t)$, of the Fourier components of the density, $\rho^{\alpha}(\mathbf{q})$, is called the intermediate scattering function (ISF) and is the standard tool for the investigation of relaxation processes on all time and length scales [197]. For an ensemble of $n \times N$ beads it is defined by 


$$
\begin{aligned}
F^{\alpha}(\mathbf{q}, t) & =\frac{1}{n N}\left\langle\rho^{\alpha}(\mathbf{q}, t) \rho^{\alpha}(-\mathbf{q}, 0)\right\rangle \\
& =\frac{1}{n N}\left\langle\sum_{i=1}^{n N} \sum_{j=1}^{n N} \delta_{\mathcal{T}(i) \alpha} \delta_{\mathcal{T}(j) \alpha} e^{-i \mathbf{q} \cdot\left(\mathbf{r}_{i}(t)-\mathbf{r}_{j}(0)\right)}\right\rangle
\end{aligned}
$$

and for $t=0$ it is identical to the static structure factor, $G(q)$, defined in Eq. (3.5). Even though $F(\mathbf{q}, t)$ is easy to obtain in simulations, most scattering experiments do not yield $F(\mathbf{q}, t)$, but instead its temporal Fourier transform, the dynamic structure factor, ${ }^{3}$

$$
S^{\alpha}(\mathbf{q}, \omega)=\int_{-\infty}^{\infty} d t F^{\alpha}(\mathbf{q}, t) e^{-i \omega t}
$$

We also define the ISF for the centers of mass of the molecules as

$$
F^{\mathrm{com}}(\mathbf{q}, t)=\frac{1}{n}\left\langle\sum_{i=1}^{n} \sum_{j=1}^{n} e^{-i \mathbf{q} \cdot\left(\mathbf{R}_{i}(t)-\mathbf{R}_{j}(0)\right)}\right\rangle,
$$

where $\mathbf{R}_{i}(t)$ denotes the center of mass position of molecule $i$ at time $t$.

A closely related quantity is the density current,

$$
\mathbf{j}^{\alpha}(\mathbf{r}, t)=\sum_{i=1}^{n N} \delta_{\mathcal{T}(i) \alpha} \mathbf{u}_{i}(t) \delta\left(\mathbf{r}-\mathbf{r}_{i}(t)\right),
$$

for particles of type $\alpha$. As before, its spatial Fourier transform is

$$
\mathbf{j}^{\alpha}(\mathbf{q}, t)=\sum_{i=1}^{n N} \delta_{\mathcal{T}(i) \alpha} \mathbf{u}_{i}(t) e^{-i \mathbf{q} \cdot \mathbf{r}_{i}(t)}
$$

The longitudinal current correlation function, $C_{\ell}^{\alpha}(\mathbf{q}, t)$, is defined similarly to $F^{\alpha}(\mathbf{q}, t)$, and it can be shown easily that both are closely connected [197],

$$
\begin{aligned}
C_{\ell}^{\alpha}(\mathbf{q}, t) & =\frac{1}{n N}\left\langle\mathbf{j}^{\alpha}(\mathbf{q}, t) \cdot \mathbf{j}^{\alpha}(-\mathbf{q}, 0)\right\rangle \\
& =-\frac{d^{2}}{d t^{2}} \frac{1}{n N}\left\langle\rho^{\alpha}(\mathbf{q}, t) \rho^{\alpha}(-\mathbf{q}, 0)\right\rangle \\
& =-\frac{d^{2}}{d t^{2}} F^{\alpha}(\mathbf{q}, t) .
\end{aligned}
$$

For later use we also need the Fourier transform of $C_{\ell}^{\alpha}(\mathbf{q}, t)$ in the time domain,

\footnotetext{
${ }^{3}$ To calculate this integral it is assumed that $F(\mathbf{q}, t)$ is an even function in $t$.
} 
$J^{\alpha}(\mathbf{q}, \omega)$. A quick look at Eq. (4.12) shows that it is related to the dynamic structure factor by

$$
J^{\alpha}(\mathbf{q}, \omega)=\int_{-\infty}^{\infty} d t C_{\ell}^{\alpha}(\mathbf{q}, t) e^{-i \omega t}=\omega^{2} S^{\alpha}(\mathbf{q}, \omega) .
$$

For convenience, we also define the sums of the previous quantities over all particle types as

$$
\begin{aligned}
\rho(\mathbf{q}, t) & =\sum_{\alpha} \rho^{\alpha}(\mathbf{q}, t) \\
F(\mathbf{q}, t) & =\frac{1}{n N}\langle\rho(\mathbf{q}, t) \rho(-\mathbf{q}, 0)\rangle, \\
S(\mathbf{q}, \omega) & =\int_{-\infty}^{\infty} d t F(\mathbf{q}, t) e^{-i \omega t}, \\
J(\mathbf{q}, \omega) & =\omega^{2} S(\mathbf{q}, \omega) .
\end{aligned}
$$

At this point a technical remark on the numerical evaluation of Eqs. (4.144.17 ) is in order. Since we are interested in the short as well as in the long time behavior of $F(\mathbf{q}, t)$, a high sampling rate for the configurations over a long interval is needed. Instead of saving the full configuration $\left\{\mathbf{r}_{i}(t)\right\}$ every few integration steps, we decided to save only the Fourier components, $\rho^{\alpha}(\mathbf{q}, t)$, which saves some time and disk space. Furthermore, we are interested in the range of small to intermediate $\mathbf{q}$, thus a large number of wave vectors is needed. Hence, it becomes very inefficient to calculate $\rho^{\alpha}(\mathbf{q}, t)$ for every $\mathbf{q}$ and every $t$ individually.

A faster method for computing the $\rho^{\alpha}(\mathbf{q}, t)$ is needed. In our case this is achieved by a Fast Fourier Transform (FFT). The two-dimensional FFT calculates the Fourier transform on a discrete set of points in real space with periodic boundary conditions and yields a discrete set of Fourier components, that are commensurable with the periodic boundary conditions. Therefore it is necessary to obtain a discretized representation of the density first, in which the particles are smeared over one or more grid points. Then it is possible to evaluate $\rho^{\alpha}(\mathbf{q}, t)$ for all $\mathbf{q}$ at once. It should be mentioned that this method of calculating $\rho^{\alpha}(\mathbf{q}, t)$ is -from a technical point of view- very similar to the calculation of the electrostatic potential with the Particle-Mesh-Ewald (PME) method that is frequently used in MD for the study of Coulomb interactions [38, 39].

Specifically, we used the assignment function devised by Essmann et al., where a very fine grid is needed and cardinal B-splines of fourth order are used to smear each particle over $4 \times 4$ grid points [38]. Here we use typical grid 
sizes of $128 \times 128$ or $256 \times 256$ for a box length of $\sim 30 r_{c}$. The FFT is then calculated with the FFTW library. Although this algorithm is very efficient, there are two sources that cause anisotropies in the data at high q. First, there is the underlying grid that destroys rotational and translational invariance on the length scale of the lattice spacing completely. Second, the assignment function is also not rotationally invariant. Since we are only interested in the regime of small to medium q, we did not try to fix these issues. However they can be attenuated by using higher order B-splines or even finer discretizations of the grid.

\subsubsection{Results I: Brownian motion}

Before turning to the collective motion, we focus on the individual motion. In this section three different systems with $\kappa N=100, \chi N=30$ are studied. The first one, $\bar{\rho}^{*}=17$, is below the main phase transition and in the gel phase $\left(L_{\beta}\right)$, while the second, $\bar{\rho}^{*}=18$, and the third, $\bar{\rho}^{*}=40$, are in the fluid phase $\left(L_{\alpha}\right)$. The systems with $\bar{\rho}^{*}=17$ and 18 mimic lipidic membranes, whereas the system with $\bar{\rho}^{*}=40$ mimics polymeric membranes. Each system contained $n=4680$ amphiphiles with $N=16$ beads, and was simulated for $5-8 \times 10^{4} \tau$ in the NVT ensemble using the standard DPD thermostat with $\gamma=0.5$. Preequilibrated, tensionless bilayers were used as initial configurations. Measurements of the MSD, the NGP, and the VACF were done every $0.02 \tau$.

\section{Mean-square displacement}

Figure 4.2 depicts the MSD of the beads averaged over all types and the MSD of the centers of mass as a function of time, as well as the running exponents. Four different regimes can be distinguished for the beads, and three different ones for the centers of mass. They are discussed in turn.

At very small times there is the ballistic regime with $\left\langle\left(\mathbf{r}_{i}(t)-\mathbf{r}_{i}(0)\right)^{2}\right\rangle \sim t^{2}$ for the beads as well as for the centers of mass. The source of this regime is the inertia of the interacting particles and the discrepancy between these two arises only from the different masses. The observation of this regime is consistent with all experiments and Eq. (4.2) yields a perfect fit.

At $t \approx 0.1 \tau$ there is a first crossover in the dynamics of the beads to a second regime scaling linearly with $t$. This regime is clearly visible from the running exponent in the interval $0.4 \tau<t<1.6 \tau$ and should not be confused with the freely diffusive one at long times, though it has the same scaling exponent. On this time scale the beads have already suffered collisions with other beads, but they have not yet realized that they are a part of a larger entity. Since the interactions are weak, the stochastic forces of the thermostat outweigh the nonbonded interactions and a net random force remains, which leads to a diffusive 


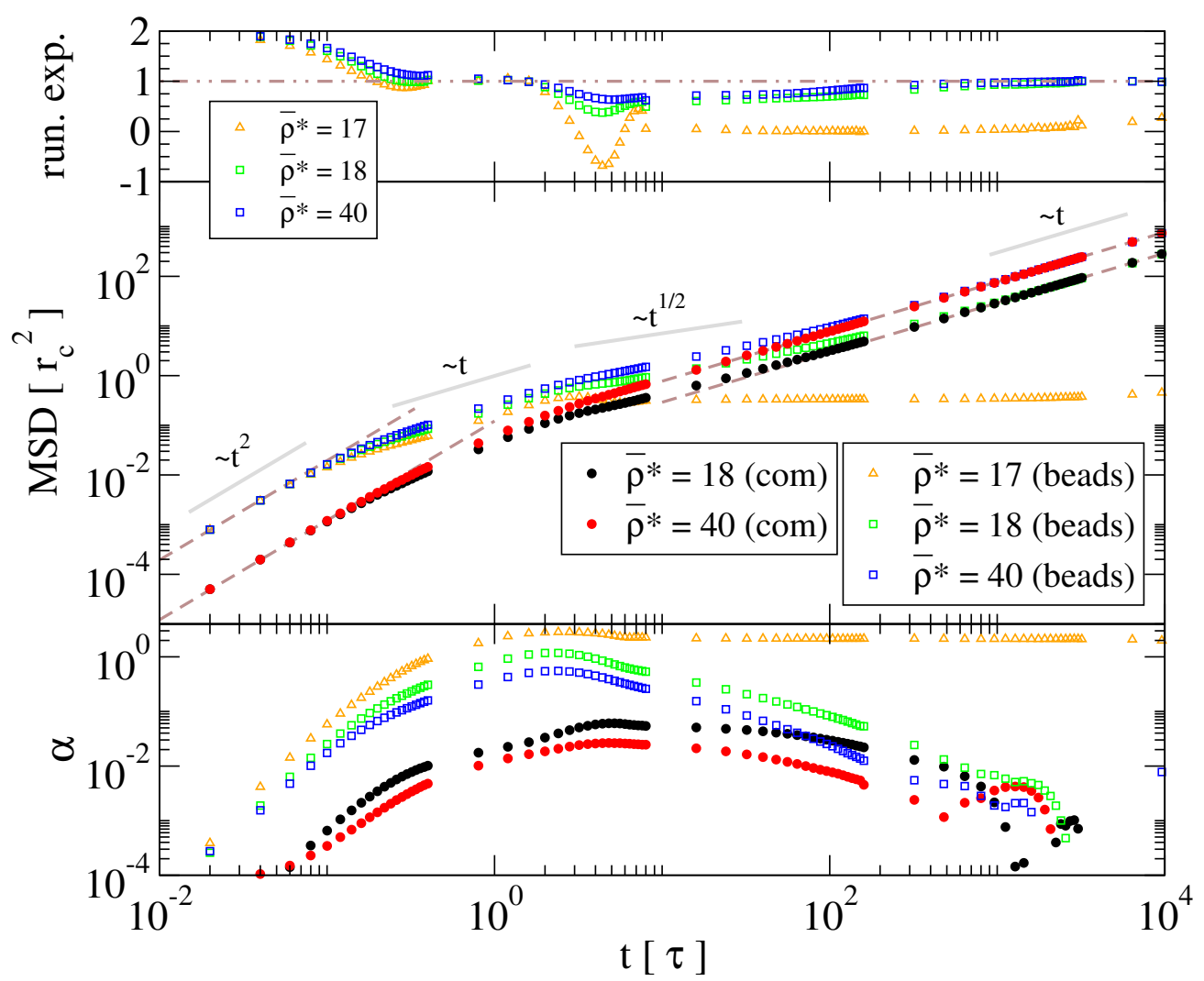

Figure 4.2: Middle: lateral MSD of the beads (open symbols) and of the centers of mass (filled symbols) for $\bar{\rho}^{*}=17,18$ and 40 . The dashed lines mark fits to the ballistic and diffusive regimes for the centers of mass at short and long times, respectively. Top: running exponent of the beads' MSD. The dashed-dotted, gray line denotes unity. Bottom: the NGP, $\alpha$, for the same systems. 
motion of the beads around the center of mass of their molecule. Hence, this regime is a consequence of the thermostat and therefore the plateaus of all three MSD curves coincide. The regime is seen neither in the MSD of the centers of mass, nor in atomistic models. ${ }^{4}$

For $t>3 \tau$ a second crossover shows up. This is where the beads realize that they cannot diffuse freely and the molecule has to diffuse as a whole, so that this regime is a consequence of the molecular connectivity. The MSDs of the beads and of the centers of mass become subdiffusive with $\left\langle\left(\mathbf{r}_{i}(t)-\mathbf{r}_{i}(0)\right)^{2}\right\rangle \sim t^{\beta}, \beta<$ 1. Qualitatively, this is similar to the Rouse model in polymer dynamics [279], although we do not observe the predicted exponent $\beta=1 / 2$. In an atomistic MD study Flenner et al. have tried to compute the scaling exponent and ended up between $\beta=0.427$ and $\beta=0.677$ [55].

For even longer times, $t>300 \tau$, the beads acquire the diffusion coefficient of the centers of mass, $D$, which is dominated by the friction of the DPD thermostat. For $\bar{\rho}^{*}=18$ and $\bar{\rho}^{*}=40$ this leads to a free diffusion, as it is described by Eq. (4.1). Here we obtain $D=7.28 \times 10^{-3} r_{c}^{2} / \tau\left(\bar{\rho}^{*}=18\right)$ and $D=19.3 \times 10^{-3} r_{c}^{2} / \tau\left(\bar{\rho}^{*}=40\right)$, respectively. For $\bar{\rho}^{*}=17$ the molecules stay where they are and start to move only on much longer time scales. Although it is difficult to measure, we obtained $D=2.6(1) \times 10^{-7} r_{c}^{2} / \tau$ for the gel phase. This result is three to four orders of magnitude smaller than in the fluid phase, but this ratio is in excellent agreement with experimental studies.

The NGP, $\alpha(t)$, differs by approximately two orders of magnitude between the beads and the centers of mass (see Fig. 4.2, bottom). For the former $\alpha(t)$ rises continuously from zero to a maximum at $t=2-3 \tau$, where $\alpha=0.5$ $\left(\bar{\rho}^{*}=40\right), 1.1\left(\bar{\rho}^{*}=18\right)$, and $2.9\left(\bar{\rho}^{*}=17\right)$. The maximum is located at the second crossover (from the diffusive to the subdiffusive regime), which indicates that a broad distribution of MSDs exist. Qualitatively this can be understood by remembering that $\alpha(t)$ was calculated by averaging over all beads, regardless of their type. Of course, the hydrophilic beads in the head groups are more mobile than the hydrophobic ones in the interior. Here, the different mobilities become very pronounced and lead to the maximum of the NGP. In the fluid phases $\alpha$ decays, because on larger time scales the motion of the whole amphiphiles dominates. However, in the gel phase the hydrophobic tails are at rest, but the head groups are still mobile. Therefore the distribution of the MSDs is essentially frozen and $\alpha$ remains constant for $\bar{\rho}^{*}=17$.

The NGP for the centers of mass is two orders of magnitude smaller than for the beads. Although there is a maximum at $t=5-6 \tau$, the NGP is always smaller than 0.06. In other two-dimensional systems of glass forming liquids $\alpha$ is typically much larger, e.g., $\alpha=0.6$ [272] or $\alpha=0.5-2$ [280, 281], so that our distribution of the MSD is virtually always Gaussian. These findings already rule out the possibility of having any significant dynamic heterogeneities.

\footnotetext{
${ }^{4}$ see for instance [55, 278]
} 


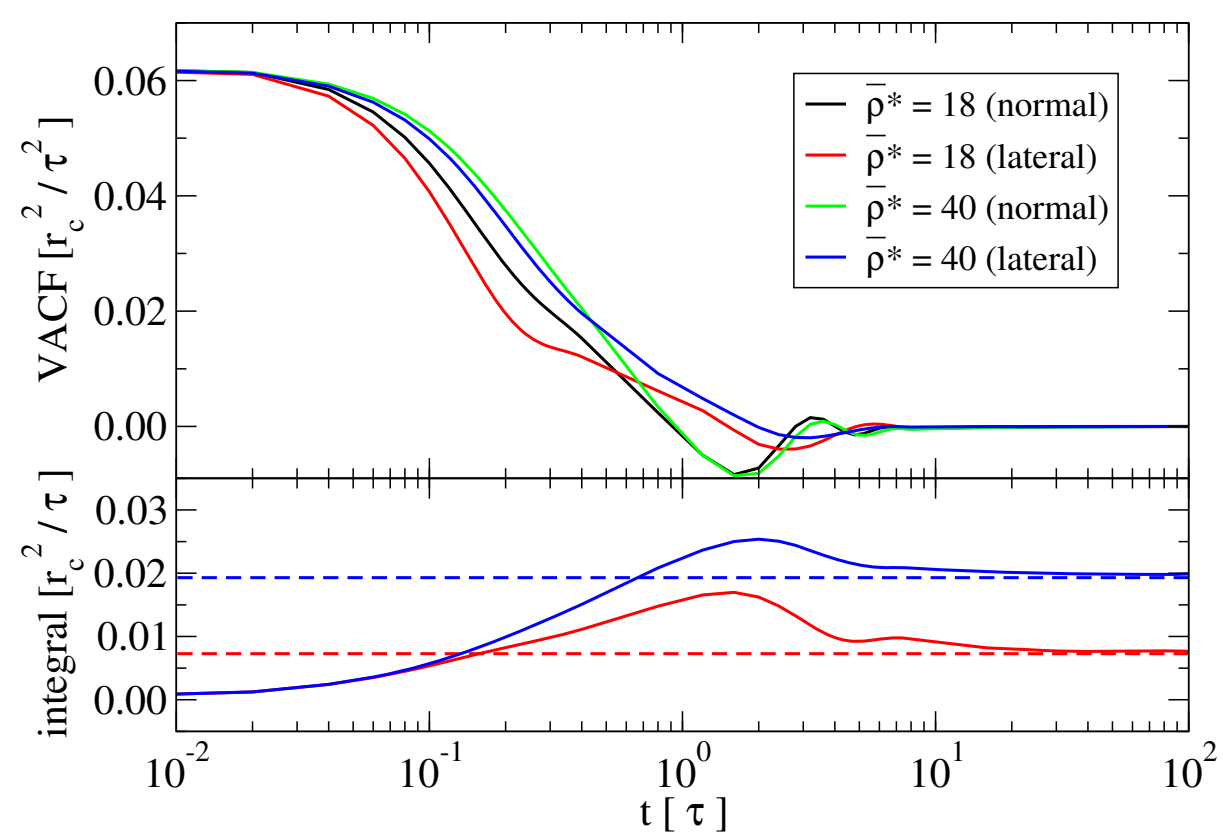

Figure 4.3: Top: VACF for the centers of mass of the two systems in the normal and in the lateral direction. Bottom: running time integral over the VACF for the lateral direction. Apparently, the integrals converge and the limiting value gives the lateral diffusion coefficient (see Eq. (4.4)). The diffusion coefficients obtained from the MSD are indicated as dashed lines.

\section{Velocity autocorrelation}

We continue the discussion of the Brownian motion with the VACF that gives an independent estimate of the diffusion coefficient via Eq. (4.4). The upper panel of Fig. 4.3 shows the VACF of the centers of mass in the lateral, but also in the normal direction for the two systems in the fluid phase. For $t=0$ they all start at the squared thermal velocity $v_{t h}^{2}=k_{B} T / N$. After an initial exponential decay the lateral VACF for $\bar{\rho}^{*}=18$ kinks at $t=0.2 \tau$. This is the time when the ballistic regime of the beads ends and crosses over to the first diffusive regime. After this time the first beads of an amphiphile have hit a nearby molecule and their velocities change the sign and decorrelate. This kink is unseeable for $\bar{\rho}^{*}=40$, because of the higher bead density. At $t=1.53 \tau\left(\bar{\rho}^{*}=18\right)$ or $t=1.97 \tau\left(\bar{\rho}^{*}=40\right)$ the VACF crosses zero and changes its sign. This can be conceived as the collision time between two amphiphiles. At this time the center of mass of the molecule springs back and the VACF decreases further until it reaches its minimum at $t=2.6 \tau\left(\bar{\rho}^{*}=18\right)$ or $t=3.3 \tau\left(\bar{\rho}^{*}=40\right)$. At this time most of the beads have already collided with other molecules so that the VACF rapidly decays and only the long time tail remains. 
The integrated VACF (lower panel of Fig. 4.3) shows a maximum where the VACF crosses zero for the first time, but then converges to a plateau whose value equals $D$. We find $D=0.0075 r_{c}^{2} / \tau\left(\bar{\rho}^{*}=18\right)$ and $D=0.0198 r_{c}^{2} / \tau$ $\left(\bar{\rho}^{*}=40\right)$. Both are in very good agreement with the diffusion constants obtained earlier from the MSD. A premature plateau indicating the "rattling in a cage" motion - as it is obtained from QENS experiments- is not seen. This is in line with the discussion of the MSD and shows that there is no small time scale in our model where the amphiphiles are confined. The convergence of the integrals to the same value as before indicates that there is no diverging long time tail in the VACF. Even though statistics are bad for long times we have the impression that a $t^{-3 / 2}$ power law fits better than a $t^{-2 / 2}$ power law.

The normal component of the VACF decorrelates approximately as fast as the lateral component, but it shows some oscillatory behavior stemming from the confinement in the normal direction. At the center of the bilayer the amphiphiles collide with the molecules from the other leaflet, whereas they are dragged back into the bilayer by the attractive interactions of their own leaflet if they move too far to the outside. This up and down motion is closely related to the protrusion modes that can be seen in the height fluctuation spectrum at high q [191, 282]. For both systems we find roughly the same frequency $\omega=3.8 \tau^{-1}$.

\section{Flipflop}

During the simulations we also looked for flipflop events in the fluid phase. To this end the normal positions of the centers of mass of the hydrophilic head groups, $x_{i}^{h}(t)$, and of the hydrophobic tails, $x_{i}^{t}(t)$, for all molecules $i$ and for all snapshots taken at time $t$ have been calculated. If $x_{i}^{h}(t)>x_{i}^{t}(t)$ a lipid is considered to be on the upper leaflet, otherwise on the lower leaflet. In our definition a flipflop event takes place, if $\left(x_{i}^{h}(t)-x_{i}^{t}(t)\right)$ switches its sign and keeps this new sign for at least $10 \tau$. This is a rather mild condition, if one keeps in mind that a flipflop would normally lead to a permanent change of the molecule's leaflet. However, even with this criterion we were unable to detect a single flipflop event neither for $\bar{\rho}^{*}=18$ nor for $\bar{\rho}^{*}=40$. Thus, we cannot give a numerical value for the flipflop rate, $r$, of our model, but only a lower boundary, ${ }^{5}$

$$
r \ll 2.67 \times^{-9} \tau^{-1} \approx 3 \mathrm{~s}^{-1} .
$$

If one keeps in mind that thermally activated flipflops naturally happen with a rate $r \approx 10^{-5} \mathrm{~s}^{-1}$, then our result is compatible with the experimental observation. However, our coarse-grained model is unable to reach these time scales, and therefore we conclude that flipflops are so rare in our model, that they can be safely neglected.

\footnotetext{
${ }^{5}$ where the conversion units for $\bar{\rho}^{*}=18$ have been used, see below.
} 


\section{Mapping of time scales}

A comparison of $D$ with experimental results (for long times) can be used to map the unit of time in our model. Typical values in lipid membranes are around $D=5 \mathrm{\mu m}^{2} / \mathrm{s}$. A comparison with the result for $\bar{\rho}^{*}=18, D=$ $7.3 \times 10^{-3} r_{c}^{2} / \tau$, and the previously mapped unit of length, $1 r_{c}=0.78 \mathrm{~nm}$ (cf. Eq. (3.19)), thus yields

$$
1 \tau=0.89 \mathrm{~ns} \quad\left(\bar{\rho}^{*}=18\right) .
$$

For the polymeric membrane, $\bar{\rho}^{*}=40$, we obtained $D=19.3 \times 10^{-3} r_{c}^{2} / \tau$ which corresponds to $0.1 \mathrm{\mu m}^{2} / \mathrm{s}$ and $1 r_{c}=2.6 \mathrm{~nm}$ (cf. Eq. (3.20)), hence

$$
1 \tau=1.3 \mu \mathrm{s} \quad\left(\bar{\rho}^{*}=40\right) .
$$

These two numbers serve as conversion factors for the unit of time in the models with $\bar{\rho}^{*}=18$ and $\bar{\rho}^{*}=40$, respectively.

\subsubsection{Results II: correlated motion}

We now turn to the study of collective dynamical phenomena in lipid bilayers where many molecules move in unison. We start at the largest length scales and then advance down to the scale of single molecules. The quantities of interest are the intermediate scattering function, $F(q, t)$, or the dynamic structure factor, $S(q, \omega)$. The former one is easier to obtain in simulations, while the latter one is the typical quantity obtained from time-resolved scattering experiments.

\section{Speed of sound}

On the largest time and length scales the bilayer behaves like a continuum and hydrodynamic equations apply. Hence, it is possible to study collective excitations, like propagating waves, dispersion relations, etc. in the framework of generalized hydrodynamics. These phenomena are interesting by themselves, but they also serve as a physical check for $F(q, t)$ and $S(q, \omega)$.

We start the discussion with an estimation of the speed of sound, $c_{s}$, of the bilayers in our model. This speed marks the upper boundary for all meaningful velocities, but particularly for the velocity of flowing, hypothetical lipid clusters. A first crude approximation of $c_{s}$ in the liquid phase can be obtained from the isothermal compressibility, $\kappa_{T}$, the molecular number density, $\rho_{c}$, and the molecular mass, $m$, by the relation $c_{s}=1 / \sqrt{\kappa_{T} \rho_{c} m}$. The product of the isothermal compressibility with the molecular density of the bilayer's hydrophobic interior has already occurred earlier in the definition of $\kappa N$ (cf. Eq. (2.7)). Since the mass of a single bead is unity in our units and only the hydrophobic 
beads participate in the sound propagation (which is shown below), $m$ equals the number of hydrophobic beads, i.e., $m=12$. Hence,

$$
c_{s}=\frac{1}{\sqrt{\kappa_{T} \rho_{c} m}}=\sqrt{\frac{\kappa N \times k_{B} T}{m}}=\sqrt{\frac{100}{12}} \frac{r_{c}}{\tau} \approx 3 \frac{r_{c}}{\tau},
$$

irrespective of the other model parameters. Transferred into physical units, this corresponds to $c_{s} \approx 3 \mathrm{~m} / \mathrm{s}\left(\bar{\rho}^{*}=18\right)$ or $c_{s} \approx 0.01 \mathrm{~m} / \mathrm{s}\left(\bar{\rho}^{*}=40\right)$, which is - even compared with the tiny value of air, $c_{s}=340 \mathrm{~m} / \mathrm{s}-$ ridiculously small. This deficiency results from the fact that the non-bonded interactions are too soft, i.e., $\kappa N$ is too small. Nevertheless, this estimate already provides a first glimpse of what one can expect from the simulations.

A more accurate estimate of $c_{s}$ can be obtained from the position of the maximum in the longitudinal current correlation function, $J(q, \omega)[57,197]$. If more than a single maximum shows up, then there are multiple longitudinally propagating dispersion relations. ${ }^{6}$

Here we compute $S(q, \omega)$ from long simulation runs of $\Delta t=12000 \tau$ where $\rho(\mathbf{q}, t)$ was computed every $0.02 \tau$, so that a trajectory of $3 \times 10^{5}$ equidistant samples was obtained. This trajectory was blocked into overlapping intervals with each one having $5 \times 10^{4}$ data points. Every interval started $1 \times 10^{4}$ data points later than its predecessor. For obtaining $S(\mathbf{q}, \omega)$ we first calculated $F(\mathbf{q}, t)$ in each interval, performed a Fourier transform in the time domain, and finally averaged over all intervals. $F(\mathbf{q}, t)$ was computed using the same recipe just omitting the Fourier transform. This intricate averaging procedure is necessary, because $S(\mathbf{q}, \omega)$ and $F(\mathbf{q}, t)$ are very noisy quantities, and a single computation lacked the desired accuracy.

Figure 4.4 shows $J(q, \omega)$ (sum of all beads), $J^{A}(q, \omega)$ (only hydrophobic beads), and $J^{B}(q, \omega)$ (only hydrophilic beads) of the fluid phase $\left(\bar{\rho}^{*}=18\right)$. In general three different peaks show up which lead to three different propagating dispersion relations, $\omega_{i}(q), i=1,2,3$ (inset of Fig. 4.4). $\omega_{1}(q)$, is defined by the position of the first peak in $J^{A}(q, \omega)$ (green dots). It has a linear dispersion over the whole interval we studied and a linear fit yields,

$$
\omega_{1}(q)=c_{1} \times q, \quad c_{1}=0.81 r_{c} / \tau .
$$

A careful analysis revealed that the corresponding peak in $S(q, \omega)$ has a Lorentzian shape, but that its FWHM scales approximately with $q$.

The hydrophilic heads possess a very similar dispersion relation, and we define $\omega_{2}(q)$ as the position of the first peak in $J^{B}(q, \omega)$. It coincides with $\omega_{1}(q)$ for $q r_{c}>1$, but bends upwards for smaller $q$. One gets the impression, that it does not even cross the origin, which would mean that this dispersion

\footnotetext{
${ }^{6}$ Peristaltic or undulatory waves do not show up because of their transverse character.
} 


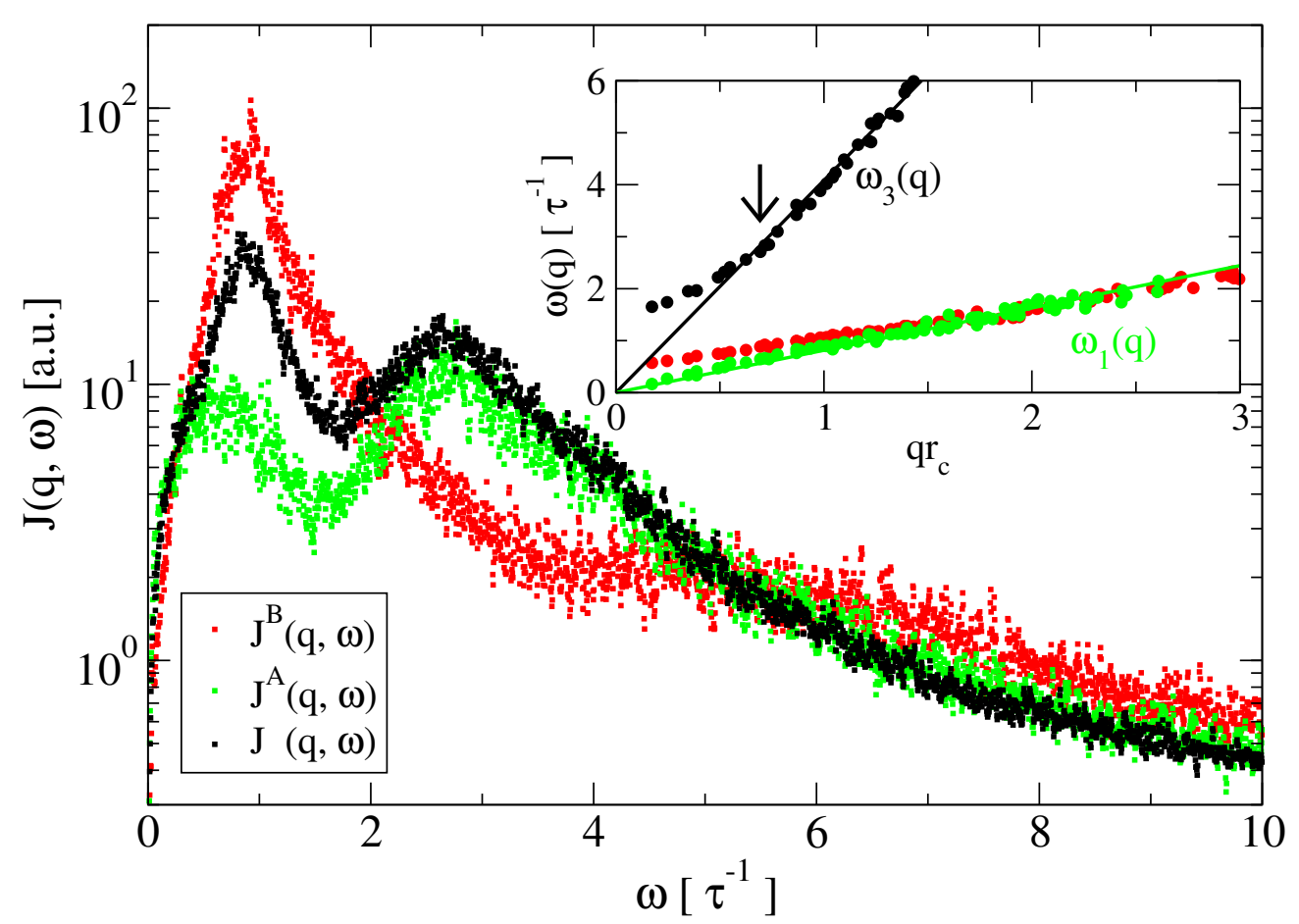

Figure 4.4: The main panel shows $J(q, \omega)$ (black), $J^{A}(q, \omega)$ (red), and $J^{B}(q, \omega)$ (green) for $\bar{\rho}^{*}=18$ ( $L_{\alpha}$ phase) and $q r_{c}=0.694$ on a logarithmic scale. Here, three propagating modes can be identified: The first peak at $\omega_{1} \tau=0.68$ stems from the hydrophobic tails and has a linear dispersion, $\omega_{1}(q)$, over the whole interval we studied (inset, green line). The hydrophilic heads possess a very similar dispersion relation, $\omega_{2}(q)$, that collapses with $\omega_{1}(q)$ for $q r_{c}>1$, but that splits off below (inset, red dots). The corresponding peak in the main panel is located at $\omega_{2} \tau=0.86$. Finally, there is a third peak at $\omega_{3} \tau=2.7$ which leads to another linear dispersion relation, $\omega_{3}(q)$. This is the sound mode and the slope of the black dots in the inset gives the speed of sound, $c_{s}$. The arrow in the inset marks the $q$-value of the main panel. 
relation is not based on a conservation law. It seems that on small scales $\omega_{1}(q)$ and $\omega_{2}(q)$ are the same or do not interact, but that they start to repel each other at $q r_{c} \approx 1$, where $\omega_{2}(q)$ splits off. However, this splitting off can only be detected if $J^{A}(q, \omega)$ and $J^{B}(q, \omega)$ are analyzed, but not the total correlation function, $J(q, \omega)$.

An explanation for the branches, $\omega_{1}(q)$ and $\omega_{2}(q)$, is currently still missing. We think that this is some kind of collective excitation where the beads of many lipids perform some internal motion coherently, similar to what one knows from atoms in a crystal with basis.

A third peak shows up at larger $q$ in $J^{A}(q, \omega)$. For $q r_{c}>1$ it has a linear dispersion, for $q r_{c}<1$ it bends also upwards. This time the width of the Lorentzian peak in $S(q, \omega)$ scales in the linear regime very well with $q^{2}$ (not shown) and we obtain

$$
\omega_{3}(q)=c_{3} \times q, \quad c_{3}=4.09 r_{c} / \tau \quad\left(\bar{\rho}^{*}=18\right) .
$$

It is well known that the dispersion relation of sound waves reads $\omega=c_{s} q$, and that the widths of the corresponding Lorentzian peaks in $S(q, \omega)$ are proportional to $q^{2}$ [197]. Therefore, we conclude that we have identified the dispersion relation of sound waves as $\omega_{3}(q)$ and that the speed of sound is given by $c_{s} \equiv c_{3}=4.09 r_{c} / \tau$. This is in good agreement with the rough estimate given in Eq. (4.21).

Finally, we calculated $J(q, \omega)$ also for the gel phase $\left(\bar{\rho}^{*}=17\right)$. We identify the same three peaks, which correspond again to three propagating dispersion relations $\omega_{i}(q)$. We start with the obvious case, $\omega_{3}(q)$ (black dots in the inset), which has a linear dispersion and a quadratic dependence on $q$ of the peak's width in $S(q, \omega)$. We obtain the speed of sound,

$$
\omega_{3}(q)=c_{3} \times q, \quad c_{3}=6.77 r_{c} / \tau \quad\left(\bar{\rho}^{*}=17\right) .
$$

This value is a factor of 2 larger than the rough estimate for the fluid phase in Eq. (4.21). This is reasonable, because it is well known that the speed of sound is larger in ordered structures than in fluids.

The other two dispersion relations, $\omega_{1}(q)$ (green dots) and $\omega_{2}(q)$ (red dots), are somewhat different than before: $\omega_{2}(q)$, the peak in $J^{B}(q, \omega)$, is more pronounced but advances with $q$ very slowly. In fact, we find an empirical dependency

$$
\omega_{2}(q)=1.02 \times\left(q r_{c}\right)^{1 / 4}
$$

An extrapolation of the branches $\omega_{2}(q)$ and $\omega_{3}(q)$ indicates that the two branches might cross at $q r_{c} \approx 0.08$, i.e., at a length scale around $80 r_{c}$. Unfortunately the system under study is too small for observing what happens at this point.

The last dispersion branch of the hydrophobic beads, $\omega_{1}(q)$, is difficult to track and ceases to exist for $q r_{c} \gtrsim 1$. Up to this point it roughly follows $\omega_{2}(q)$. 


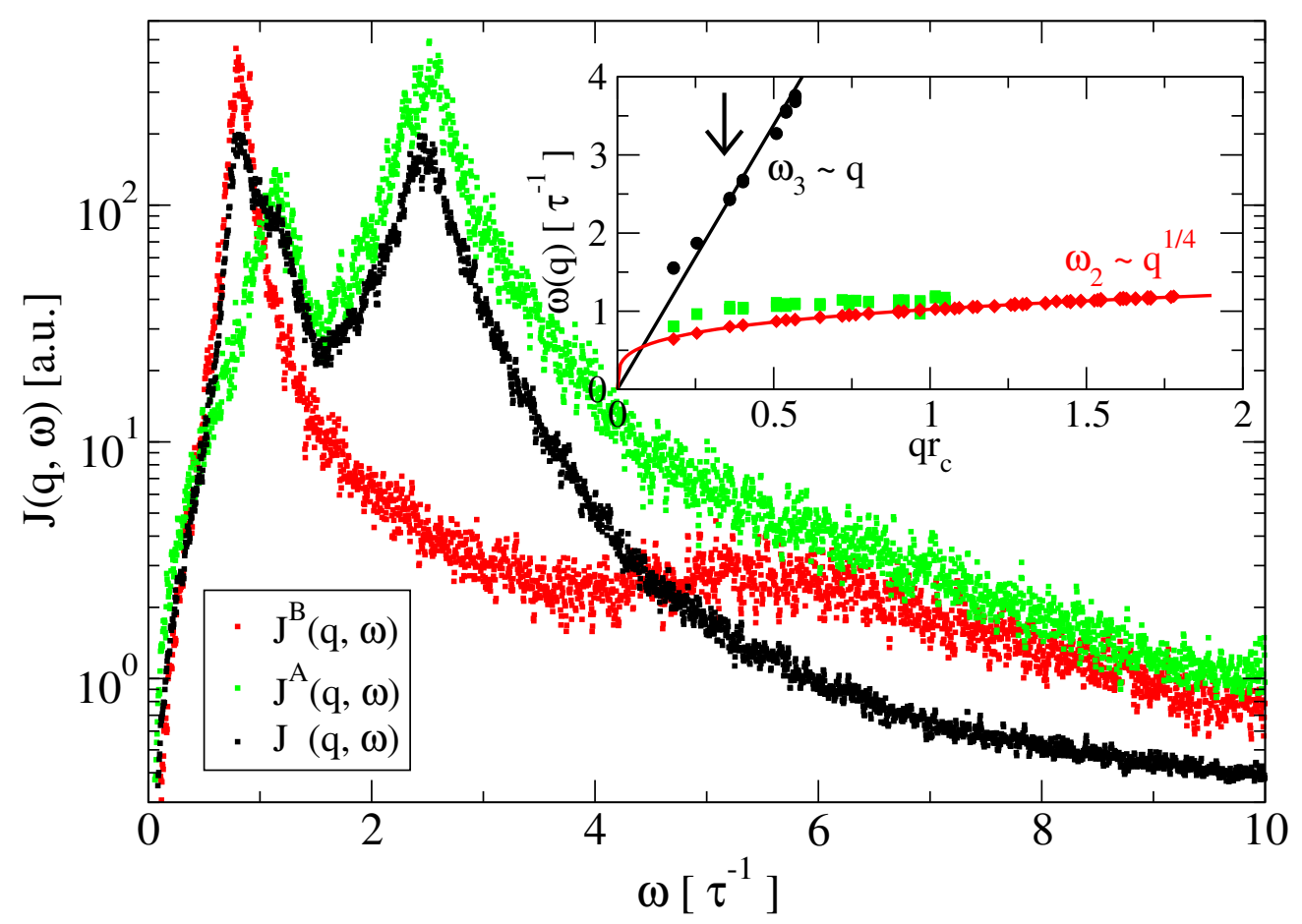

Figure 4.5: The main panel shows $J(q, \omega)$ (black), $J^{A}(q, \omega)$ (green), and $J^{B}(q, \omega)$ (red) for $\bar{\rho}^{*}=17$ ( $L_{\beta}$ phase) and $q r_{c}=0.359$ on a logarithmic scale. Three propagating modes can be identified: The first peak at $\omega \tau=0.79$ stems from the head groups and has a dispersion relation $\omega_{2} \sim q^{1 / 4}$ (inset, red line). Next there is a smaller peak at $\omega_{1} \tau=1.10$ stemming purely from the tails, but not exclusively from the trailing beads. Its dispersion is very noisy and ceases before crossing $\omega_{2}(q)$ (inset, green squares). The third mode at $\omega_{3} \tau=2.47$ is the sound or Brillouin mode with a linear dispersion, $\omega_{3} \sim q$ (inset, black line). The arrow in the inset marks the $q$-value of the main panel. 


\section{Structural relaxation}

Another collective phenomenon is the structural relaxation of the lipids' lateral coordination, i.e., the time decay of the intermolecular packing peak in the static structure factor, $G(q)$. This has been the topic of several time-resolved scattering experiments and it is generally accepted that two decay processes are involved, a fast and a slow one [257, 265]. Here we analyze the initial decay of $F(q, t) / F(q, 0)$ for $\bar{\rho}^{*}=18$ and $\bar{\rho}^{*}=40$ in the time interval $0<t<30 \tau$ and the $q$-interval $5<q r_{c}<13$. We use the same results as before and employ a phenomenological fitting function,

$$
f(t)=A_{1} e^{-t^{2} / 2 s^{2}}+A_{2} e^{-t / \tau_{i}}
$$

where the Gaussian component accounts for random thermal fluctuations and all fast processes; the exponential term takes care of the slow structural relaxation. $F(\mathbf{q}, t) / F(\mathbf{q}, 0)$ is fitted for each $\mathbf{q}$-vector with four fitting parameters: $A_{1}, A_{2}$ are dimensionless prefactors and $\tau_{i}, s$ are characteristic time scales for each decay. The sum $A_{1}+A_{2}$ serves as a test for the quality of the fit and its value should be in all cases around unity. ${ }^{7}$ Smaller values of $q$ cannot be fitted with this approach, because of the oscillatory behavior of $F(\mathbf{q}, t)$ as a function of $t$ coming from the propagating waves. This is particularly severe in the gel phase where propagating waves make $F(q, t)$ strongly oscillatory even for high $q$.

The obtained fitting parameters are displayed in Fig. 4.6 as a function of $q r_{c}$ for both systems. The sum of the prefactors, $A_{1}+A_{2}$, is nearly constant and always deviates from unity by less than $7 \%$. A multiplication of each prefactor with $G(q)$ yields a decomposition of the packing peak into two differently decorrelating components (upper row in Fig. 4.6). It turns out that the exponentially decaying one has the largest contribution to the peak $(\sim 75 \%)$, and that the longest relaxation times $\tau_{i} \approx 1.06 \tau\left(\bar{\rho}^{*}=18\right)$ and $0.48 \tau\left(\bar{\rho}^{*}=40\right)$ coincide with the position of the peak at $q r_{c} \approx 9.3$. The Gaussian component has its maximum contribution at slightly smaller $q$, i.e., $q r_{c}=8.3\left(\bar{\rho}^{*}=18\right)$ or $8.1\left(\bar{\rho}^{*}=40\right)$ and its maximum decay time, $s$, at $q r_{c}=9.0\left(\bar{\rho}^{*}=18\right.$ and $\bar{\rho}^{*}=40$ ). The peak in $G(q)$ mainly originates from the hydrophobic beads (cf. Sec. 3.1.4), so that the exponentially decaying contribution can be attributed to the relaxation of the lipids' tails. The Gaussian component stems from the tails as well as from the heads.

Looking at the amplitude of the exponentially decaying component, $A_{2}$, one gets the impression that it is a single peak overlaid with a second exponentially

\footnotetext{
${ }^{7}$ It is always slightly larger than unity, because the initial decay on the scale of the integration time step of any time correlation function is Gaussian due to the microscopic reversibility of the integration algorithm. Ignoring this Gaussian decay and directly assuming an exponential decay leads to a small overestimation of the initial amplitude.
} 


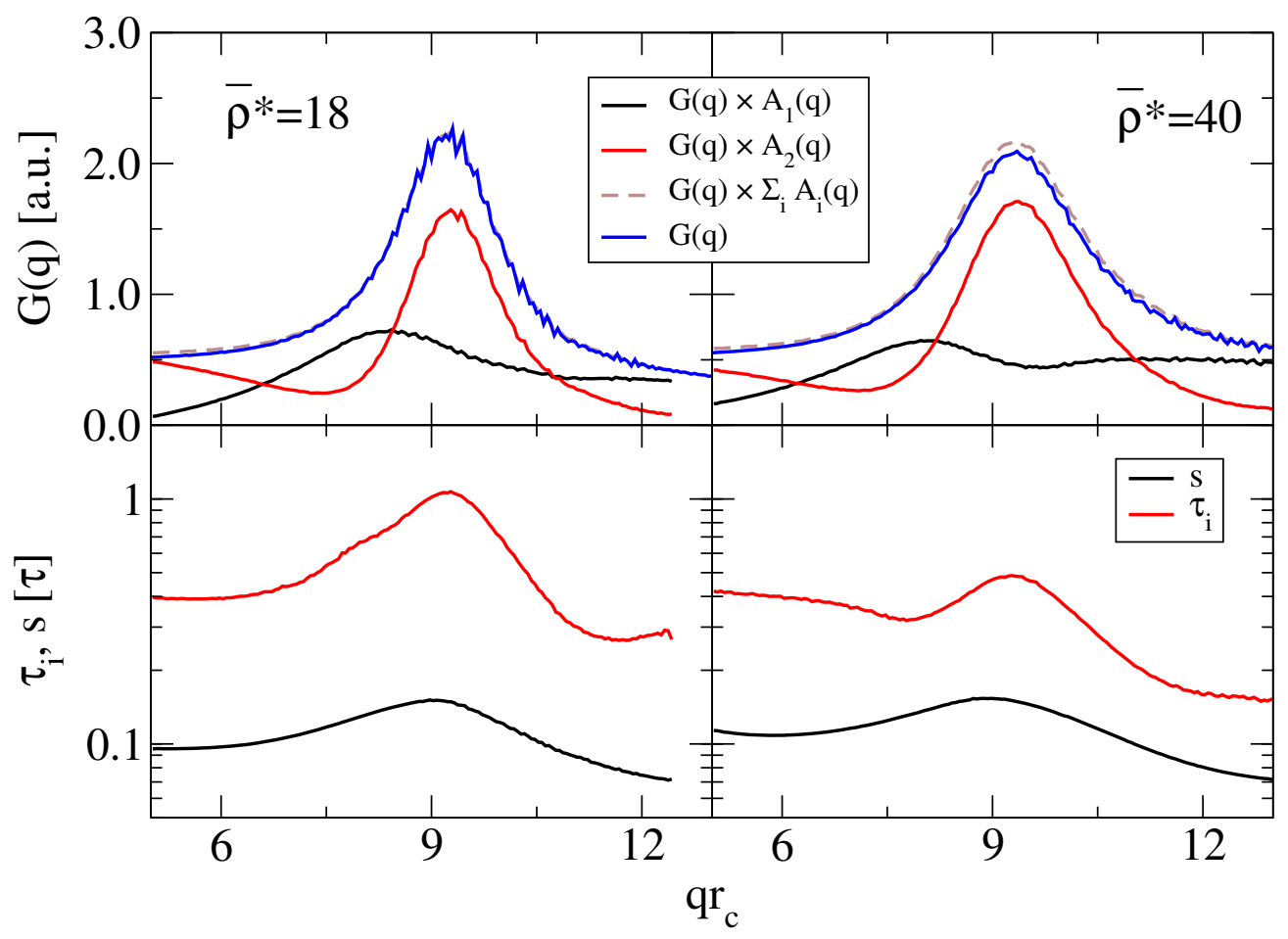

Figure 4.6: Structural relaxation of the fluid phase at $\bar{\rho}^{*}=18$ (left) and $\bar{\rho}^{*}=40$ (right) obtained by fitting $F(q, t) / F(q, 0)$ to Eq. (4.26). Top: static structure factor, $G(q)=F(q, 0)$, of the fluid phase (blue) with the packing peak reflecting the characteristic length scale that separates two amphiphiles. The amplitudes of the two components, $A_{1}(q)$ and $A_{2}(q)$, (black, red) are scaled with $G(q)$ so that the absolute contributions to the scattering intensity become visible. $G(q) \times\left(A_{1}(q)+A_{2}(q)\right.$ ) (dashed gray line) agrees very well with $G(q)$. Bottom: relaxation rates, $s, \tau_{i}$, (black, red). 
decaying process at smaller $q$. One does not get the impression that this peak continues to smaller $q$, which would be essential for a longer ranged correlation in space and time. Instead, if the second process is ignored, the peak looks like a Lorentzian. Its width, $\Gamma$, gives an estimate of the correlation length, $\xi \simeq \Gamma^{-1}$. We find $\xi_{18} \approx 1 / 1.52 r_{c}=0.66 r_{c}\left(\bar{\rho}^{*}=18\right)$ and $\xi_{40} \approx 1 / 2.00 r_{c}=0.50 r_{c}$ $\left(\bar{\rho}^{*}=40\right)$.

If we compare the obtained time and length scale of this relaxation process with the neutron scattering results from Rheinstädter et al., we find partial agreement: they state that "the lipid tails displacements are correlated for at least four lipid diameters between $t_{1}[=2.5 \mathrm{ps}]$ and $t_{2}[=1.1 \mathrm{~ns}]$ " [265]. In the lipidic system, $\bar{\rho}^{*}=18$, we find a similar time scale $\tau_{i} \approx 1.05 \tau=0.93 \mathrm{~ns}$, but a shorter correlation length $\xi_{18}=0.5 \mathrm{~nm}$, which is approximately only one lipid diameter. This means that the time scale agrees very well, but that the spatial extension of dynamic correlations is significantly shorter than in experiments. It is in any case much shorter than the $10 \mathrm{~nm}$ found by Falck et al. using atomistic MD [92]. Thus after moving a lipid diameter, each molecule has already forgotten about its previous situation. In case of the polymeric system, $\bar{\rho}^{*}=40$, we find $\tau_{i} \approx 0.48 \tau=0.62 \mu \mathrm{s}$.

\section{Flow motion}

We now turn to the correlated motion of the centers of mass and to the discussion of the flow pattern that Falck et al. observed. Since this pattern has been observed in atomistic MD on a length scale of $10 \mathrm{~nm}$ and a time scale of 10 ns, we need a larger system than before, but we do not need to simulate it for a long time. Here, we report the simulation results of a system with now $n=74880$ lipid molecules and a box length of $L_{y}=L_{z}=144.814 r_{c} \approx 113 \mathrm{~nm}$. The interaction parameters are the same as before, but now we focus on lipid bilayers with $\bar{\rho}^{*}=18$. The system has been assembled from preequilibrated pieces of tensionless bilayers and has been simulated for $3600 \tau$.

A direct visualization of the center of mass displacements, as it is shown by Falck et al. [92], is in our case inconclusive because the displacements are blurred by a lot of thermal noise, and averaging over different snapshots is unhelpful since $\left\langle\mathbf{v}_{i}\right\rangle=0$. Therefore we omit the discussion of such displacement maps and concentrate on the study of $F^{\mathrm{com}}(q, t)$ in which all intramolecular motion is left aside.

To analyze the data we take the idea from Busch et al. that the lipid clusters move ballistically on the time scale of 1 ns [257]. This motion is similar to that of beads at very short times before they suffer collisions (cf. Eq. (4.2)), but with a velocity $v_{0} \ll v_{t h}$. The expected collective motion manifests itself as a Gaussian distribution in $q$ as well as in $t$, 


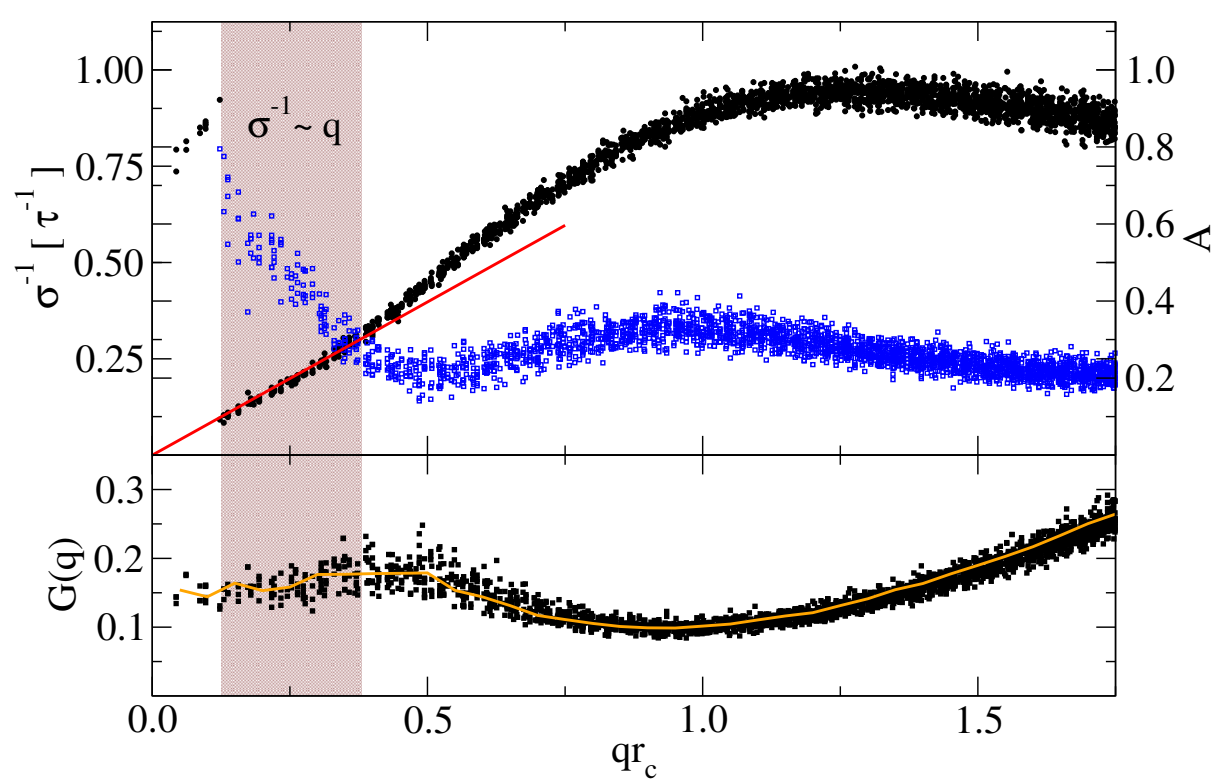

Figure 4.7: Top: the initial decay of $F^{\mathrm{com}}(\mathbf{q}, t) / F^{\mathrm{com}}(\mathbf{q}, 0)$ has been fitted to Eq. (4.28). The black dots denote $\sigma^{-1}(\mathbf{q})$, the blue squares $A(\mathbf{q})$. The shaded interval marks the area in which $\sigma^{-1} \sim q$, i.e., where large patches of lipids move ballistically. The red line is a linear fit to $\sigma^{-1}(\mathbf{q})$. Bottom: $G(\mathbf{q})$ of the centers of mass (black squares) and its radial average $G(q)$ (orange line). $G(q)$ has a broad maximum at $0.3<q r_{c}<0.5$.

$$
F^{\mathrm{com}}(q, t) \sim \exp \left(-\frac{q^{2} v_{0}^{2} t^{2}}{2}\right)
$$

To find this motion, we fitted $F^{\mathrm{com}}(\mathbf{q}, t) / F^{\mathrm{com}}(\mathbf{q}, 0)$ for every wave vector $\mathbf{q}$ to the sum of a Gaussian and an exponential decay,

$$
\frac{F^{\mathrm{com}}(\mathbf{q}, t)}{F^{\operatorname{com}}(\mathbf{q}, 0)} \simeq A(\mathbf{q}) \exp \left(-\frac{t^{2}}{2 \sigma(\mathbf{q})^{2}}\right)+[1-A(\mathbf{q})] \exp \left(-\frac{t}{t_{0}(\mathbf{q})}\right)
$$

with a variance $\sigma(\mathbf{q})^{2}$, an amplitude $A(\mathbf{q})$, and an arbitrary decay time $t_{0}(\mathbf{q})$ in the time interval $0 \ldots 10 \tau$. The exponential decay is included to cover other non-Gaussian decays at short times as well. A comparison of Eq. (4.28) with Eq. (4.27) shows that one expects $\sigma^{-1}=q v_{0}$ in the region where clusters move ballistically. In other words the decay time, $\tau_{\mathbf{q}}=1 /\left(q v_{0}\right)$, of the corresponding modes of the ISF grow linearly with increasing size, $\Delta x=2 \pi / q$, i.e., $\tau_{\mathbf{q}} \sim$ $1 / q \sim \Delta x$.

The fitted $\sigma^{-1}(\mathbf{q})$ and $A(\mathbf{q})$ are shown in the upper panel of Fig. 4.7, the lower panel depicts $G(q)$. The fits succeeded for all but the smallest $\mathbf{q}$, where 
the time interval was probably too narrow for observing a Gaussian decay. The expected scaling, $\sigma^{-1} \sim q$, holds in the interval $0.125<q r_{c}<0.38$; for $q r_{c}>1.0, \sigma^{-1}$ first levels off and then decreases slowly. Interestingly, the matching interval roughly coincides with a small dip in $G(q)$ between $q=0.3$ and $0.5 r_{c}^{-1}$, which indicates the existence of structures on this length scale. The broad nature of this dip shows that these have a size between 12 and $21 r_{c}$ $(9-16 \mathrm{~nm})$.

We fitted a line through the origin to $\sigma^{-1}$ in the first interval and obtained $v_{0}=0.8 r_{c} / \tau=3.2 v_{t h}$. This velocity is a factor of five smaller than the speed of sound (Eq. (4.23)), but it is still a factor of three larger than the thermal velocity. According to the Maxwell-Boltzmann statistics the probability of finding a lipid with a velocity $\left\|\mathbf{v}_{i}\right\| \geq v_{0}$ is around $0.1 \%$. This means that there are by far not enough lipids in the membrane to form ballistically moving clusters of such a size. Hence, the motion is too fast for being a flow conveying lipids.

A possible explanation for these findings comes from the unidentified propagating branch of the dispersion relation, $\omega_{1}(q)$, Eq. (4.22): the velocity $v_{0}$ matches nicely the velocity $c_{1}=0.81 r_{c} / \tau$ found earlier. The observed dip in $G(q)$ gives us a rough estimate of the typical wave length of this collective excitation.

\subsubsection{Conclusions}

The overall goal of this long but important section was to answer the question if our model is able to shed some light on the recent discussion of the lipids' diffusion process. To this end we have discussed many dynamic properties of the fluid and the gel phase. The discussion started with the motion of individual beads and lipids by inspecting the mean-square displacement. The MSD has many of the features (a ballistic, a subdiffusive regime, and a freely diffusive regime) that are also seen in atomistic simulations, but the DPD thermostat modifies the bead's dynamics on short time scales. We do not find evidence for a jump-diffusion like motion. The discussion of the velocity autocorrelation function also showed that there is no "rattling in a cage" motion of the lipids.

We continued the discussion with the non-Gaussianity parameter and found that the only noteworthy dynamic heterogeneity comes from the difference of the heads and the tails, which is not of particular interest. In case of the centers of mass, there was hardly any deviation from a Gaussian distribution of the mean-square displacements, so that we can rule out the possibility of having dynamic heterogeneities or some kind of string-like motion in our model. ${ }^{8}$ All lipids diffuse roughly at the same time scale.

\footnotetext{
${ }^{8}$ We did observe dynamic heterogeneities in some non-equilibrium situations, like the spinodal decomposition of a gel phase into a fluid phase.
} 
After this first part of the discussion it was already clear that the motion of the lipids is more like that of polymers in a dense melt, where collective effects are of minor relevance. However, we carried on to see which kinds of concerted motion there are. We measured the intermediate scattering function and the dynamic structure factor to obtain the dispersion relations and with them the spectrum of collective excitations of the bilayer. Several branches exist in the fluid as well as in the gel phase, and we could estimate the speed of sound which is orders of magnitude slower than in experiments, due to the softness of our interactions.

An analysis of the structural relaxations showed that the typical correlation length is on the order of one lipid diameter, so that collective relaxations are unlikely to occur. However, the time scale of these relaxations agrees nicely with neutron scattering experiments and is on the order of $1 \mathrm{~ns}$.

Even though the situation is almost clear, we followed the idea of Busch et al., that lipid clusters would flow ballistically if they existed [257]. Surprisingly, we have found the expected scaling regime in the decay of the intermediate scattering function. It turned out that this motion is too fast for being a flow conveying lipids. Hence, this finding rules out the possibility of having a ballistic flow of lipids in our model on the probed time scale.

Taking all results into account, the following picture of a lipid's motion emerges: At very short times it moves ballistically. After $\sim 0.1 \tau$, it hits another molecule in the vicinity which slows it down and causes its conformation to change. After $\sim 1 \tau$ the lipid has already suffered so many collision, that the conformation has decorrelated completely, but its center of mass has not moved very far, yet. At $10 \tau$, the velocity of its center of mass is uncorrelated from its initial value, and after $100 \tau$ the molecule performs a lateral random walk. There is no support for a dynamic formation of lipid clusters, a strongly cooperative diffusion, or even a collective flow of lipids in our model.

In this section we have learnt a lot about the motion of the beads and the molecules in our model. We have found many similarities but also some differences to atomistic models, and we have shown that in our model Brownian motion dominates over collective motion.

\subsection{Surface viscosity and intermonolayer friction}

It is known from the earliest experiments on red blood cells that these "flicker" under the microscope. This flickering was also observed in other cells and liposomes, and was related to a continuous undulation of the surface. In the first analytical treatment of this shape relaxation processes, the bilayer was treated as a thin sheet surrounded by a viscous solvent that dissipated the energy of the undulations [283]. The analytical predictions were confirmed by 
video microscopy experiments, but it turned out that the applicability of this theory was restricted to large length scales where the molecular character of the bilayer is negligible. On much smaller scales, the lipids diffuse within the monolayer, slide past one another causing friction in a monolayer, and another dissipation mechanism, the surface viscosity of the fluid monolayers, becomes important.

It was pointed out by Evans et al. that there is a third dissipative process on intermediate scales [31]. If the two monolayers of a bilayer laterally move against each other, their relative motion is damped by a frictional force (see Fig. 4.8). This intermonolayer friction cannot be treated in the simple picture of a structureless, thin sheet membrane anymore, but it crucially depends on the existence of two adjacent monolayers. Its incorporation into the analytical treatment led together with the surface viscosity to an understanding of the relaxation processes on all length scales, and is known as the Seifert-Langer (SL) theory [84, 85].

Measuring these dissipation mechanisms is a delicate issue and experiments are rare. All of the experiments focussing on the surface viscosity, $\eta$, are based on a theory by Saffmann [284, 285] where the diffusion of a tracer particle in a membrane surrounded by a solvent is related to $\eta$. The first measurements were done with membrane tethers [286], but many other objects like latex spheres [287-289], large proteins [290], or even entire lipid domains [291] have been used as tracer particles over the years. Typical values for $\eta$ in liposomes are in the range of $1-10 \times 10^{-10} \mathrm{~Pa} \mathrm{~m} \mathrm{~s}^{9}{ }^{9}$ Values for polymersomes are approximately a factor of 500 higher [292].

The intermonolayer friction coefficient, $b$, was measured first in a bilayer where the bottom monolayer was fixed to a substrate and the diffusion of a tracer particle was tracked [293]. Most of the newer experiments rely on membranes where large curvatures occur, like in pulling a thin tether from a vesicle [31, 32, 294, 295], in fusion pores [296], or in chemical instabilities that trigger the ejection of tubules [297]. The time correlations of undulations in bilayer stacks or giant vesicles were also used for measuring $b$ [298-300]. The experimental results for lipid bilayers are in the range of $1-10 \times 10^{8} \mathrm{~Pa} \mathrm{~s} / \mathrm{m},{ }^{10}$ for polymeric membranes they are about an order of magnitude larger [292].

In computer simulations little attention has been given to $b$ and $\eta$. A notable exception is a series of articles from the Briels group studying both quantities within a coarse-grained model with explicit solvent [301-304]. $\eta$ is extracted from a Green-Kubo relation (GK) as well as from non-equilibrium molecular dynamics (NEMD) with Lees-Edwards sliding boundary conditions [44, 305], where perpendicular shear is applied to the bilayer. $b$ is obtained from NEMD

\footnotetext{
${ }^{9}$ Frequently, $\eta$ is reported in units of "surface poise", where $1 \mathrm{SP}=10^{-3} \mathrm{Pams}$

${ }^{10} b$ is often reported in cgs units: 1 dyne $\mathrm{s} \mathrm{cm}^{-3}=10 \mathrm{~Pa} \mathrm{~s} / \mathrm{m}$
} 

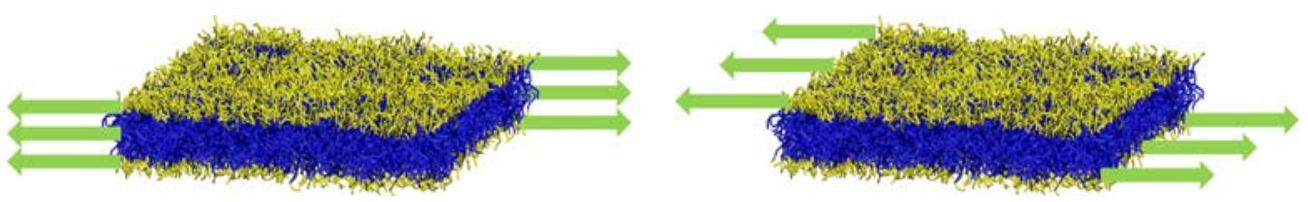

Figure 4.8: The two shear modes in bilayer membranes. Following the convention on sheared block copolymers [307], they are denoted as perpendicular shear (left) and parallel shear (right). The resistance of the bilayer against perpendicular shear is characterized by the surface viscosity, $\eta$, whereas its resistance against parallel shear is characterized by its intermonolayer friction coefficient, $b$. Forces are shown as green arrows.

runs with parallel shear (see Fig. 4.8) and also through comparison with the SL theory $[84,85]$. However, their results for $b$ and $\eta$ are two orders of magnitude too small compared to the experimental counterpart. Additionally, they observe deviations from the predictions of the SL theory in the autocorrelation function of the undulation modes which they cannot explain. But since their results for $b$ are in close proximity to a value reported in an atomistic study [278], they come to the conclusion that "the interpretation of the experimental data appears as the most likely source of the discrepancy" [304]. The same model has also been used by other authors to mimic one of the experiments mentioned above, where $b$ was extracted from the diffusion of a large protein [306].

To shed further light on these issues, we decided to measure $b$ and $\eta$ in our coarse-grained solvent-free model. It turns out that the procedure for measuring $b$ is complicated by the lack of solvent and none of the procedures used so far in simulations is accurate for our model. On the one hand sliding boundary conditions cannot be used in the case of a solvent-free model, because parallel shear (cf. Fig. 4.8) is needed and in that case the sliding boundary conditions must have been applied directly at the head groups of the bilayer, which would lead to a significant distortion of the bilayer. On the other hand, the original SL theory crucially depends on the presence of a viscous solvent which is absent in solvent-free models. The aim of the present section is therefore to establish techniques for measuring $b$ and $\eta$ that are independent of the solvent viscosity, to apply them, and in this way to gain a deeper insight into the dynamics of pure bilayers.

\subsubsection{Theory}

In this work we utilize two different methods for obtaining the surface viscosity of a single monolayer, $\eta$, and two different methods for obtaining the intermonolayer friction coefficient, $b$. 


\section{A Green-Kubo relation for $\eta$}

The first relation for $\eta$ is the well-known Green-Kubo (GK) formula [308, 309] that relates the autocovariance of the off-diagonal elements of the momentary pressure tensor, $P_{\alpha \beta}(t)$, to $\eta$ by

$$
\eta=\frac{V L_{x}}{2 k_{B} T} \int_{0}^{\infty} d t^{\prime}\left\langle P_{y z}\left(t^{\prime}\right) P_{y z}\right\rangle .
$$

The factor 2 in the denominator originates from the fact that the viscosity of a monolayer is calculated. The derivation of this equation is based on two conservation laws: the conservation of the particle number and the conservation of the momentum. It also depends on the corresponding continuity equations that connect the temporal change in the density field with the momentum density and the temporal change of the momentum density with the stress tensor [197]. $P_{y z}(t)$ is computed with the virial theorem [44].

\section{Reverse Non-Equilibrium Molecular Dynamics (RNEMD)}

Alternatively, we obtain $\eta$ from a set of RNEMD simulations [310-312]. Briefly, the idea in these simulations is to generate an artificial flow of lipids and to measure the resulting velocity profile. Compared to standard NEMD simulations this scheme represents a reversal of the cause and the effect - the velocity gradient and the lipid flow, respectively. To this end, the bilayer was divided in the $y$-direction into 20 stripes numbered from 1 to 20 . After every fixed time interval $\Delta t_{s}$ the most positive $z$-component of a particle's velocity in stripe 1 was exchanged with the most negative $z$-component of another particle's velocity residing in stripe 11 . The resulting perpendicular shear flow is damped by viscous forces which heat the system. If this perturbation is weak enough, the temperature and the density gradients become negligible, and $\eta$ is obtained from

$$
\eta=\frac{P_{\mathrm{tot}}}{4 t L_{z}\left|\frac{\partial v_{z}}{\partial y}\right|},
$$

where $P_{\text {tot }}$ is the total momentum exchanged during the time $t$ and $\partial v_{z} / \partial y$ is the velocity gradient measured in the simulation.

\section{A Green-Kubo relation for $b$}

If parallel shear is applied to a bilayer, the velocity profile within each monolayer is nearly constant and the monolayers are moving with equal, but opposite velocities causing a frictional force, $F$, at the midplane of the bilayer. For small velocities one expects that $F$ is proportional to the contact area, $A$, and to the 
velocity difference between the monolayers, $\Delta v$. The constant of proportionality is $b$. Hence,

$$
F=A \times \Delta v \times b .
$$

For measuring $b$ we derive one more GK relation, which is very similar to an equation used in hydrodynamics to calculate friction coefficients of surfaces [313, 314]. In linear-response theory, the parallel shear can be described by a perturbation Hamiltonian $\mathcal{H}^{\prime}=\mathcal{H}^{+}+\mathcal{H}^{-}$where

$$
\mathcal{H}^{ \pm}= \pm \frac{v_{0}}{2} \sum_{i=1}^{n N^{ \pm}} p_{i y}
$$

is the perturbation acting on the upper $(+)$ or lower leaflet $(-)$, respectively. The sum runs over all $n N^{ \pm}$particles in each leaflet and $v_{0}$ is a small velocity. This perturbation creates a constant flow in the $+y$-direction on the upper and in the $-y$-direction on the lower leaflet. The velocity profile up to first order in $v_{0}$ is

$$
v_{y}^{ \pm}= \pm \frac{v_{0}}{2}
$$

Linear-response theory yields the non-equilibrium average of any variable $B$ in the presence of this perturbation as

$$
\langle B(t)\rangle_{\mathrm{NE}}-\langle B\rangle_{0}=\frac{v_{0}}{k_{B} T} \int_{-\infty}^{t} d s\langle B(t-s) \dot{A}(s)\rangle
$$

where the subscript "NE" denotes a non-equilibrium average where the time evolution is controlled by the perturbed Hamiltonian; the subscript "0" denotes an equilibrium average without the perturbation [197]. Here, we use $A=$ $\mathcal{H}^{\prime} / v_{0}=\left(\sum_{i=1}^{n N^{+}} p_{i, y}-\sum_{i=1}^{n N^{-}} p_{i, y}\right) / 2$. Hence,

$$
\dot{A}=\frac{1}{2}\left(F_{y}^{+}-F_{y}^{-}\right)=F_{y}^{+},
$$

where Newton's third law was used in the second equality. $F_{y}^{ \pm}=\sum_{i} F_{y, i}$ are the $y$-components of the total forces that are exerted on the upper or lower monolayer, respectively. If we substitute $F_{y}^{+}$for $B$ and introduce

$$
b=\frac{1}{A k_{B} T} \int_{0}^{\infty} d t\left\langle F_{y}^{+}(t) F_{y}^{+}\right\rangle
$$

then Eq. (4.34) can be rewritten in the final form using $\Delta v=v_{y}^{+}-v_{y}^{-}=v_{0}$, $\left\langle F_{y}^{+}\right\rangle_{0}=0$, and Eq. (4.35) as 


$$
\left\langle F_{y}^{+}\right\rangle_{\mathrm{NE}}=A \times \Delta v \times b,
$$

which is identical to Eq. (4.31). Unlike the GK relation for $\eta$, this one is not based on a conservation law.

\section{Undulation mode autocorrelation}

It was realized by Seifert and Langer that $b$ manifests itself through the decay of the autocorrelation function of the undulatory modes [84, 85]. In their model the bilayer consists of two thin sheets with a bending rigidity, $\kappa$, surrounded by a viscous solvent with a shear viscosity, $\eta_{S}$. The lipid densities on the upper $(+)$ or the lower (-) leaflet at the neutral surface ${ }^{11}$ are denoted as $\phi^{ \pm}$, respectively (see Fig. 4.9). In a plane bilayer they equal the projected densities at the midplane, $\psi^{ \pm}$, but in an undulating, curved bilayer they differ. The position of the midplane, $h(\mathbf{r})$, is specified in the Monge gauge as a height above some reference plane and the mean curvature of nearly flat membranes, $H$, is related to this position by $2 H=\nabla^{2} h(\mathbf{r})$. If $d$ denotes the normal distance between the neutral surface and the bilayer midplane, then this difference is in first order of $H$ given by

$$
\phi^{ \pm} \approx \psi^{ \pm}(1 \pm 2 d H) .
$$

The elastic free energy density of each leaflet is $\left(k_{m} / 2\right)\left(2 \phi^{ \pm} / \Phi_{0}-1\right)^{2} \approx\left(k_{m} / 2\right) \times$ $\left(\rho^{ \pm} \pm 2 d H\right)^{2}$ where $k_{m}$ is the area compressibility of a monolayer and $\rho^{ \pm}=$ $\left(\psi^{ \pm} / \Phi_{0}-1\right)$ is the scaled deviation of the projected density from the equilibrium lipid density of a flat membrane, $\Phi_{0} / 2=n / 2 A$. Together with the usual bending term of the bilayer, the free energy is

$$
F=\int d A\left(\frac{\kappa}{2}(2 H)^{2}+\frac{k_{m}}{2}\left[\left(\rho^{+}+2 d H\right)^{2}+\left(\rho^{-}-2 d H\right)^{2}\right]\right) .
$$

A Fourier expansion of $h(\mathbf{r})$ with $h(\mathbf{r})=\sum_{\mathbf{q}} h_{\mathbf{q}} \exp (-i \mathbf{q} \cdot \mathbf{r})$ and $\mathbf{q}=2 \pi \times$ $\left(n_{x} / \sqrt{A}, n_{y} / \sqrt{A}\right), n_{x}, n_{y} \in \mathbb{N}$ yields

$$
F=\frac{A}{2} \sum_{\mathbf{q}}\left(\begin{array}{lll}
h_{\mathbf{q}} & \rho_{\mathbf{q}}^{\Delta} & \rho_{\mathbf{q}}^{\Sigma}
\end{array}\right)\left(\begin{array}{ccc}
\tilde{\kappa} q^{4} & -2 k_{m} d q^{2} & 0 \\
-2 k_{m} d q^{2} & 2 k_{m} & 0 \\
0 & 0 & 2 k_{m}
\end{array}\right)\left(\begin{array}{c}
h_{\mathbf{q}} \\
\rho_{\mathbf{q}}^{\Delta} \\
\rho_{\mathbf{q}}^{\Sigma}
\end{array}\right)^{*},
$$

where the Fourier coefficients, $h_{\mathbf{q}}$, can be considered as the independent degrees of freedom. $\tilde{\kappa}=\kappa+2 d^{2} k_{m}$ is a renormalized bending rigidity and the asterisk denotes complex conjugation. $F$ is given in terms of the density difference,

\footnotetext{
${ }^{11}$ The neutral surface is the surface inside a monolayer that is neither stretched nor compressed [200].
} 


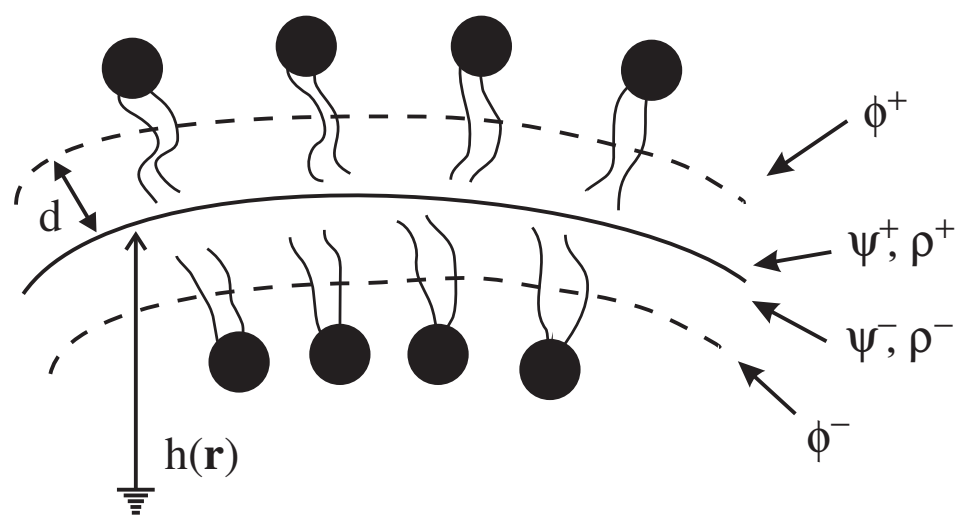

Figure 4.9: Schematic picture of a lipid bilayer. The black circles with the wavy lines represent lipid molecules, the dashed lines represent the neutral surfaces and the solid line the bilayer midplane. The lipid densities at the neutral surfaces are $\phi^{ \pm}$, and their projections onto the midplane are $\psi^{ \pm}$. The height of the midplane above some reference plane is given by $h(\mathbf{r})$.

$\rho_{\mathbf{q}}^{\Delta} \equiv\left(\rho_{\mathbf{q}}^{+}-\rho_{\mathbf{q}}^{-}\right) / 2$, and the average density, $\rho_{\mathbf{q}}^{\Sigma} \equiv\left(\rho_{\mathbf{q}}^{+}+\rho_{\mathbf{q}}^{-}\right) / 2$ which decouples, so that it is ignored in the following.

If the bilayer is excited by a single plane wave, $h(\mathbf{r})=h \exp [-i \mathbf{q} \cdot \mathbf{r}]+$ c.c., with an amplitude $h$, and $\rho^{ \pm}(\mathbf{r})=\left(\rho^{\Sigma} \pm \rho^{\Delta}\right) \exp [-i \mathbf{q} \cdot \mathbf{r}]+$ c.c., the exerted normal force density is

$$
-\frac{1}{A} \frac{\delta F}{\delta h^{*}}=-\tilde{\kappa} q^{4} h+2 k_{m} d q^{2} \rho^{\Delta} .
$$

The in-plane surface pressure, $\sigma^{ \pm}$, is in this case

$$
\sigma^{ \pm}=-\frac{1}{A} \frac{\delta F}{\delta \rho^{ \pm *}}=k_{m} \rho^{ \pm} \mp d q^{2} k_{m} h .
$$

Unfortunately, the treatment of Seifert and Langer is not directly applicable to the case of a solvent-free model, because they use a no-slip boundary condition between the solvent and the bilayer which is no longer meaningful. Simply putting $\eta_{S} \rightarrow 0$ in their expressions does not lead to well-defined expressions either, so that parts of their calculation must be reworked before it can be applied to a solvent-free model.

To determine the dynamic equation of motion for the membrane, we simply require that all normal forces balance. The central difference in this work to Seifert and Langer's work is that the out-of-plane inertia is no longer neglected. Hence,

$$
m \Phi_{0} \ddot{h}=-\frac{1}{A} \frac{\delta F}{\delta h^{*}}=-\tilde{\kappa} q^{4} h+2 k_{m} d q^{2} \rho^{\Delta}
$$


where $m$ is the mass of a lipid. The lipids in each monolayer are treated as a two-dimensional, incompressible liquid with velocities $\tilde{\mathbf{v}}^{ \pm}$, where the tilde refers to two-dimensional quantities. Within the two monolayers, the lateral force balance equation reads

$$
-\tilde{\nabla} \sigma^{ \pm}+\eta \tilde{\nabla}^{2} \tilde{\mathbf{v}}^{ \pm} \mp b\left(\tilde{\mathbf{v}}^{+}-\tilde{\mathbf{v}}^{-}\right)=0
$$

The terms on the left hand side are (i) the surface pressure, (ii) the viscous damping by the surface viscosity, and (iii) the intermonolayer friction that penalizes a relative motion between the two monolayers. The in-plane inertia can be safely neglected, because the motion of the lipids in a dense bilayer is overdamped on the time scales of interest. The densities, $\rho^{ \pm}$, obey an approximate continuity equation, $\partial_{t} \rho^{ \pm} \approx-\tilde{\nabla} \cdot \tilde{\mathbf{v}}^{ \pm}$, because flip-flops are neglected on the time scale of interest. This is certainly true for the experimental system and also approximately true for our coarse-grained model. To incorporate the continuity equations we take the divergence of Eq. (4.43), transform to the reciprocal space, and find

$$
q^{2} \sigma^{ \pm}+\eta q^{2} \dot{\rho}^{ \pm} \pm b\left(\dot{\rho}^{+}-\dot{\rho}^{-}\right)=0 .
$$

Subtracting the equations for both signs, using Eq. (4.41), and dividing by 2 we finally arrive at

$$
\left(\eta q^{2}+2 b\right) \dot{\rho}^{\Delta}=-k_{m} q^{2} \rho^{\Delta}+d q^{4} k_{m} h .
$$

With the standard way of reducing the degree of a differential equation by increasing the number of coupled equations, a combination of Eq. (4.42) and Eq. (4.45) leads to the following system of three coupled, linear, first order differential equations

$$
\partial_{t}\left(\begin{array}{c}
h \\
\rho^{\Delta} \\
\dot{h}
\end{array}\right)=-\left(\begin{array}{ccc}
0 & 0 & -1 \\
-\frac{k_{m} d q^{4}}{2 b+\eta q^{2}} & \frac{k_{m} q^{2}}{2 b+\eta q^{2}} & 0 \\
\frac{\tilde{\kappa} q^{4}}{m \Phi_{0}} & -\frac{2 k_{m} d q^{2}}{m \Phi_{0}} & 0
\end{array}\right)\left(\begin{array}{c}
h \\
\rho^{\Delta} \\
\dot{h}
\end{array}\right) .
$$

To simplify the notation, we introduce a time scale $\tau_{r}$ and a frequency $\omega_{h}$,

$$
\tau_{r}=\frac{2 b+\eta q^{2}}{k_{m} q^{2}} \quad \omega_{h}=\sqrt{\frac{\kappa q^{4}}{m \Phi_{0}}} .
$$

Additionally the renormalization of the bending rigidity, $\tilde{\kappa}$, is only a small perturbation to $\kappa$ in our case, so that we can write $\tilde{\kappa}=\kappa(1+\varepsilon), \varepsilon=2 d^{2} k_{m} / \kappa \ll$ 1. For instance, using $\bar{\rho}^{*}=18, d \approx t_{h} / 4=1.6 r_{c}, k_{m}=1.04 k_{B} T / r_{c}^{2}$, and $\kappa=18 k_{B} T$, where $t_{h}$ is the thickness of the bilayer, we obtain $\varepsilon \approx 0.3$. With 
the introduction of the parameter $P=2 k_{m} / q^{2} \kappa$, Eq. (4.46) becomes

$$
\partial_{t}\left(\begin{array}{c}
h \\
\rho^{\Delta} d \\
h / \omega_{h}
\end{array}\right)=-\left(\begin{array}{ccc}
0 & 0 & -\omega_{h} \\
-\varepsilon / P \tau_{r} & 1 / \tau_{r} & 0 \\
(1+\varepsilon) \omega_{h} & -\omega_{h} P & 0
\end{array}\right)\left(\begin{array}{c}
h \\
\rho^{\Delta} d \\
h / \omega_{h}
\end{array}\right) .
$$

The physical meaning of $\tau_{r}$ and $\omega_{h}$ becomes clear if one neglects the two coupling terms $-\varepsilon / P \tau_{r}$ and $-\omega_{h} P$ between $h$ and $\rho^{\Delta}$ for a moment. In this case the matrix in Eq. (4.48) becomes antidiagonal and we find an exponential relaxation of $\rho^{\Delta}$ with the characteristic time $\tau_{r}$ and an undamped harmonic oscillation of $h$ with frequency $\omega_{h}$. Hence, the system has one real eigenvalue, $1 / \tau_{r}$, and two purely imaginary ones, $\pm i \omega_{h}$.

An exact solution of the whole system of coupled differential equations (for instance by a Laplace transform) is possible but involves the roots of a cubic polynomial and leads to cumbersome expressions which are hard to interpret. Alternatively, one may try a perturbation calculation in which the harmonic oscillation in $h$ is weakly coupled to the relaxation in $\rho^{\Delta}$. Unfortunately, only one $\left(-\varepsilon / P \tau_{r}\right)$ of the two coupling terms in Eq. (4.48) is small, so that a straightforward expansion in $\varepsilon$ becomes impossible.

Therefore we restrict our focus on the eigenvalues, $\lambda$, of the system in Eq. (4.48) which correspond to up to three different dispersive modes. The characteristic polynomial is cubic in $\lambda$, and reads

$$
\lambda^{3}-\lambda^{2} / \tau_{r}+\omega_{h}^{2}(1+\varepsilon) \lambda-\omega_{h}^{2} / \tau_{r}=0 .
$$

Exact expressions for the roots of this polynomial exist, but from a physical point of view they are difficult to understand.

Even without solving for the roots one can calculate the discriminant of this polynomial by inserting realistic values for all parameters. This analysis reveals that one expects in virtually all cases only one real and two complex conjugated roots. Unlike the uncoupled system, the two complex roots are not purely imaginary anymore, but contain real contributions due to the coupling to the relaxation of $\rho^{\Delta}$. The real root leads to an exponential decay with a decay constant $\gamma_{1}$, while the complex roots lead to a damped, oscillatory decay with a damping constant $\gamma_{2}$ and a frequency $\omega_{B}$. Hence, an alternative parameterization of the characteristic polynomial is given by:

$$
\begin{aligned}
0 & =\left[\lambda-\gamma_{1}\right]\left[\lambda-\left(\gamma_{2}+i \omega_{B}\right)\right]\left[\lambda-\left(\gamma_{2}-i \omega_{B}\right)\right] \\
& =\lambda^{3}-\left(\gamma_{1}+2 \gamma_{2}\right) \lambda^{2}+\left(\gamma_{2}^{2}+\omega_{B}^{2}+2 \gamma_{1} \gamma_{2}\right) \lambda-\gamma_{1}\left(\gamma_{2}^{2}+\omega_{B}^{2}\right)
\end{aligned}
$$

A comparison of the coefficients of Eq. (4.49) and Eq. (4.51) leads to

$$
\tau_{r}^{-1}=\gamma_{1}+2 \gamma_{2}
$$




$$
\begin{aligned}
\omega_{h}^{2}(1+\varepsilon) & =\gamma_{2}^{2}+\omega_{B}^{2}+2 \gamma_{1} \gamma_{2} \\
\omega_{h}^{2} / \tau_{r} & =\gamma_{1}\left(\gamma_{2}^{2}+\omega_{B}^{2}\right),
\end{aligned}
$$

which are exact within the model. $b$ is contained in $\tau_{r}$ and can be obtained without any free parameters by measuring $\gamma_{1}, \gamma_{2}$, and $\omega_{B}$ either from Eq. (4.52) or Eq. (4.54).

For later reference we also provide the first order inverse expressions,

$$
\begin{aligned}
\gamma_{1} & =\frac{1}{\tau_{r}}\left(1-\varepsilon \frac{\omega_{h}^{2} \tau_{r}^{2}}{1+\omega_{h}^{2} \tau_{r}^{2}}\right)+\mathcal{O}\left(\varepsilon^{2}\right) \\
\gamma_{2} & =\frac{1}{2 \tau_{r}}\left(\varepsilon \frac{\omega_{h}^{2} \tau_{r}^{2}}{1+\omega_{h}^{2} \tau_{r}^{2}}\right)+\mathcal{O}\left(\varepsilon^{2}\right) \\
\omega_{B}^{2} & =\omega_{h}^{2}\left(1+\varepsilon \frac{\omega_{h}^{2} \tau_{r}^{2}}{1+\omega_{h}^{2} \tau_{r}^{2}}\right)+\mathcal{O}\left(\varepsilon^{2}\right),
\end{aligned}
$$

which are remarkable because they explicitly show how the coupling combined $\tau_{r}$ and $\omega_{h}$ to form three new time scales, $\gamma_{1}, \gamma_{2}$, and $\omega_{B} \cdot \gamma_{1}$ resembles the time scale of the relaxation of $\rho^{\Delta}$ and is a bit smaller than $1 / \tau_{r}$ so that differences in the density decay slower than in the uncoupled case. $\gamma_{2}$ is the new damping rate of the oscillations and is of first order in $\varepsilon$, i.e., one expects to see damped oscillatory behavior in the correlation functions. The prefactor $1 / 2$ can be understood by the fact that originally there were two oscillatory modes and the coupled damping splits between these modes giving each the same weight. The zeroth order term of the new oscillation frequency, $\omega_{B}$, is given by $\omega_{h}$. The resubstitution of $\tau_{r}, \omega_{h}$, and $\varepsilon$ in these expressions is not particularly enlightening, but it should be noted that for $q r_{c} \ll 1$

$$
\begin{aligned}
\gamma_{1} & \sim q^{2}, \\
\gamma_{2} & \sim q^{2}, \\
\omega_{B}^{2} & \sim q^{4} .
\end{aligned}
$$

The standard way of obtaining $\gamma_{1}, \gamma_{2}$, and $\omega_{B}$ involves the calculation of the autocorrelation function of $h(t)$. With an analytical expression for $h(t)$ one would calculate $C(q, t)=\left\langle h(t) h^{*}\right\rangle$ as an ensemble average over the three initial conditions $h(0), \dot{h}(0)$, and $\rho^{\Delta}(0)$. But since such an expression for $h(t)$ is currently unavailable, we propose an alternative, phenomenological expression for $C(q, t)$, which is motivated by the previous findings,

$$
C(q, t) \equiv\left\langle h(t) h^{*}\right\rangle=\left\langle|h|^{2}\right\rangle\left(A_{1} e^{-\gamma_{1} t}+A_{2} \cos \left(\omega_{B} t+\varphi\right) e^{-\gamma_{2} t}\right) .
$$

Here the weighting factors $A_{1}$ and $A_{2}$ are chosen so that $A_{1}+A_{2} \cos \varphi=$ 1 with an arbitrary phase shift, $\varphi$. The first term in Eq. (4.61), i.e., the 
simple exponential decay, reflects the relaxation of the density difference and corresponds to a single, stationary dispersive mode. The Fourier transform of such an exponential decay leads to a Lorentzian peak at $\omega=0$ in the dynamic undulation structure factor, $S(q, \omega)$. Following the convention from light scattering of simple liquids [197], this peak will be denoted as the Rayleigh line. The second term in $C(q, t)$ comes from the two complex roots which correspond to two propagating dispersive modes and lead to two Lorentzian peaks at $\omega= \pm \omega_{B}(q)$ in $S(q, \omega)$. They will be denoted as Brillouin lines. Hence, the Fourier transform of $C(q, t)$ is

$$
\begin{gathered}
S(q, \omega)=\frac{A_{R}}{\pi} \frac{\Gamma_{R} / 2}{\omega^{2}+\left(\Gamma_{R} / 2\right)^{2}}+\frac{A_{B}}{\pi} \frac{\Gamma_{B} / 2}{\left(\omega-\omega_{B}\right)^{2}+\left(\Gamma_{B} / 2\right)^{2}}+\ldots \\
\cdots+\frac{A_{B}}{\pi} \frac{\Gamma_{B} / 2}{\left(\omega+\omega_{B}\right)^{2}+\left(\Gamma_{B} / 2\right)^{2}},
\end{gathered}
$$

where the index $R$ denotes the Rayleigh line and the index $B$ denotes the Brillouin line of the propagating mode. $\Gamma_{R}$ and $\Gamma_{B}$ are the FWHM of the two Lorentzians, and $A_{R}$ and $A_{B}$ are prefactors. The connection to Eq. (4.61) is given by $\Gamma_{R} / 2=\gamma_{1}$ and $\Gamma_{B} / 2=\gamma_{2}$.

\subsubsection{Results and discussion}

Six systems in the fluid phase with $\bar{\rho}^{*}=18,20,25,30,35$, and $40, \kappa N=100$, and $\chi N=30$ were simulated to measure $\eta$ and $b$ by the GK relations as well as $b$ from the undulation mode autocorrelation. Each system contained $n=4680$ amphiphiles with $N=16$ beads and was simulated for $2-5 \times 10^{4} \tau$ in the NVT ensemble using the standard DPD thermostat with $\gamma=0.5$. Preequilibrated bilayers under vanishing lateral tension were used as initial configurations.

\section{Measurement of the surface viscosity}

For measuring $\eta$ with the GK formula, Eq. (4.29), the elements of the pressure tensor, $P_{\alpha \beta}$, were saved every $0.005 \tau$, i.e., in every integration step. $P_{y z}(t)$ is blocked into overlapping intervals with a length of $250 \tau$, each block starting $50 \tau$ after the start of the previous one. $\left\langle P_{y z}(t) P_{y z}\right\rangle$ is computed in each block and averaged (Fig. 4.10, lower panel). The integral in Eq. (4.29) over the block-averaged $\left\langle P_{y z}(t) P_{y z}\right\rangle$ was evaluated numerically. For $t$ between 20 and $75 \tau$ a first plateau showed up (Fig. 4.10, upper panel), i.e., $\left\langle P_{y z}(t) P_{y z}\right\rangle$ reached approximately zero. This point determines the cutoff of the integral in Eq. (4.29). Generally speaking, $\eta$ will have a larger statistical uncertainty if the integral is continued after this point because one integrates up mostly noise. Frequently, the choice of the onset of the first plateau was ambiguous so 


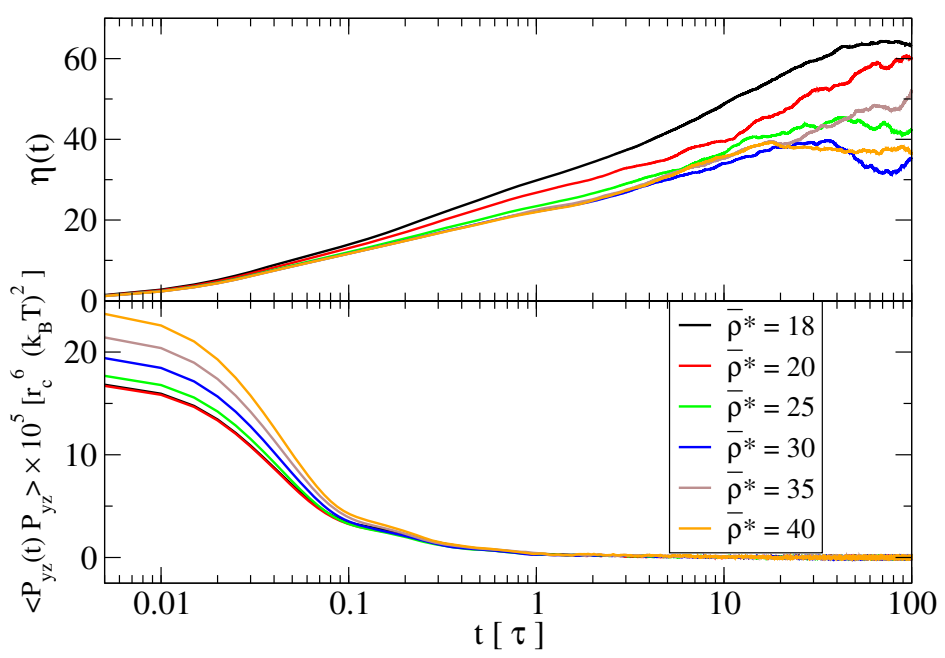

Figure 4.10: Lower panel: Block-averaged autocovariance of an off-diagonal element of the pressure tensor, $P_{y z}$, for different $\bar{\rho}^{*}$. Upper panel: Running integral over the autocovariances with the prefactors from Eq. (4.29), so that $\eta(t) \rightarrow \eta$ in the limit $t \rightarrow \infty$.

that a systematic error was introduced at this point. The statistical error was obtained from a blocking analysis [315]. This yielded the values reported for $\eta$ in Tab. 4.1 in the column "Green-Kubo".

Alternatively, $\eta$ was computed using the RNEMD methodology from the data of dedicated simulations lasting $2-4 \times 10^{4} \tau$. The bilayer was divided into 20 stripes along the $y$-direction and the velocities in the $z$-direction were swapped between the stripes 1 and 11. Five bead velocities were swapped at once every $0.5 \tau$. The total exchanged momentum $P_{\text {tot }}$ was saved every $2 \tau$.

The velocity gradient $\partial v_{z} / \partial y$ and its statistical error were obtained from a simultaneous linear regression of both velocity profiles in the unperturbed region around $v_{z}=0$ (Fig. 4.11, bottom). Additionally, the lipid number density profile, $\Phi(y) / \Phi_{0}$, normalized to its equilibrium value $\Phi_{0}$, as well as the temperature profile in the $y$-direction have been computed (top, middle in Fig. 4.11). The total exchanged momentum per time, $P_{\text {tot }} / t$, was obtained by fitting the saved values of $P_{t o t}$ to a line through the origin. The statistical error in this quantity was so low, that it could be safely neglected. The final value of $\eta$ was obtained by Eq. (4.30), the statistical error from the usual error propagation equations. The results for $\eta$ are shown in Tab. 4.1 in the column "RNEMD".

The rate of velocity swaps is set on purpose to a small value, so that the perturbation of the equilibrium properties is negligible, but the velocity profile is linear in the center of the shear zones. Tenney and Maginn derive analytic 


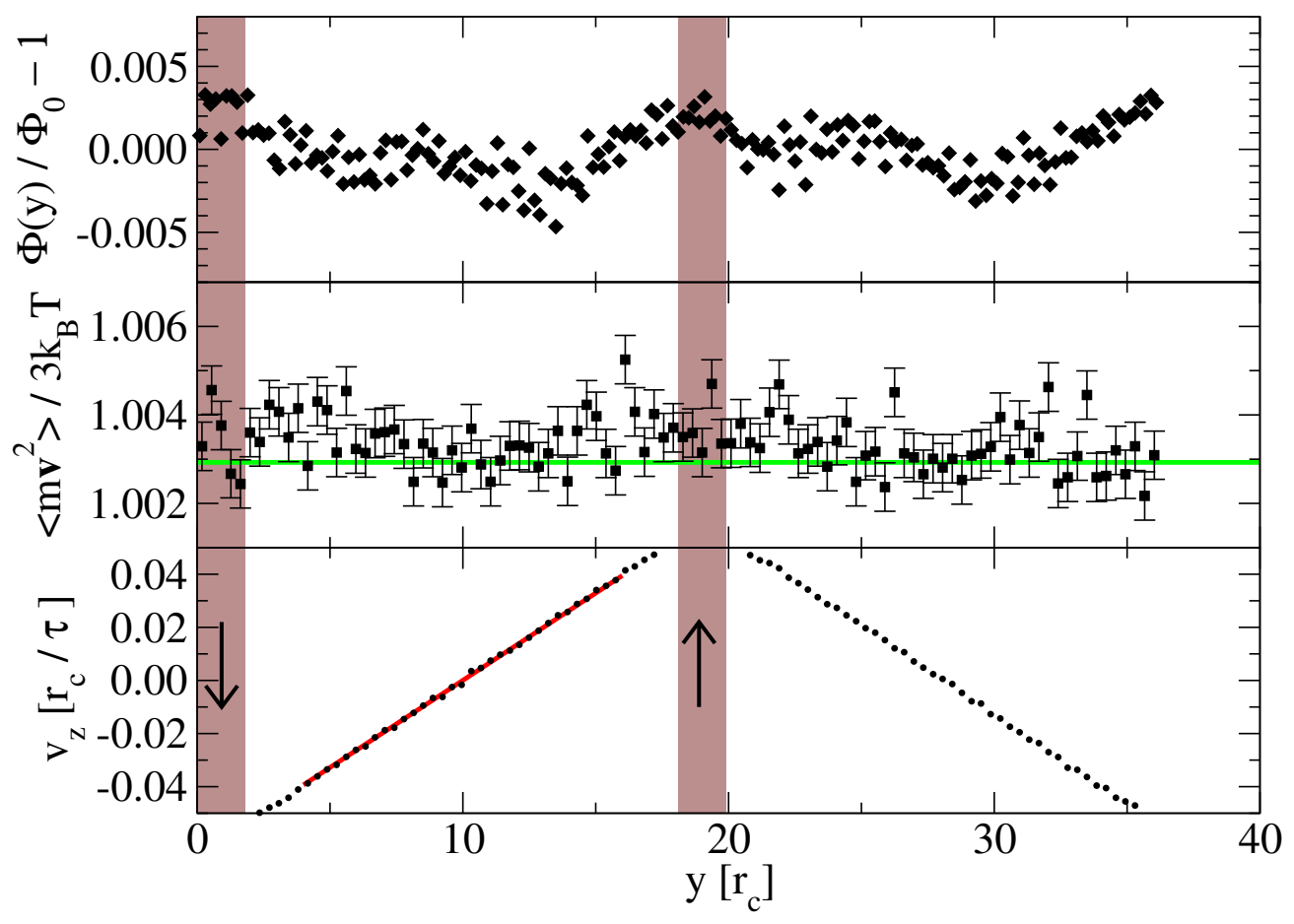

Figure 4.11: Top: lipid areal density profile as a function of $y$ for $\bar{\rho}^{*}=18$ recorded during the RNEMD runs. The profile is normalized by the average density $\Phi_{0}$. The gray shaded areas mark the two stripes, in which the velocities are swapped. Middle: average kinetic energy per degree of freedom, i.e., temperature. The green line marks the average temperature in a DPD simulation without the RNEMD perturbation. Bottom: velocity profile in the $z$-direction. The red line marks the linear fit to the profile in the center of the shear zones. The arrows visualize the direction of lipid flow.

Table 4.1: Surface viscosities and intermonolayer friction coefficients

\begin{tabular}{ccccc}
\hline \hline $\bar{\rho}^{*}$ & \multicolumn{2}{c}{$\eta\left[k_{B} T \times \tau \times r_{c}^{-2}\right]$} & \multicolumn{2}{c}{$b\left[k_{B} T \times \tau \times r_{c}^{-4}\right]$} \\
& Green-Kubo & RNEMD & Green-Kubo & Undulations \\
\hline 18 & $63(3)$ & $66.2(7)$ & $1.7(1)$ & $0(1)$ \\
20 & $57(6)$ & $56.7(5)$ & $2.0(1)$ & - \\
25 & $43(4)$ & $48.1(5)$ & $2.9(1)$ & - \\
30 & $37(3)$ & $43.7(6)$ & $4.3(2)$ & - \\
35 & $46(3)$ & $42.4(5)$ & $6.0(2)$ & $8(1)$ \\
40 & $37(3)$ & $42.4(3)$ & $7.6(4)$ & - \\
\hline \hline
\end{tabular}


expressions for the density and the temperature profiles inside the shear zones for a bulk fluid which are quadratic in $y$ [311]. In contrast to our simulations they use the thermostat only during the equilibration phase and disable it before the actual RNEMD phase starts. Thus, energy and momentum are conserved and the velocity swaps lead to a viscous heating of the system. They solve a heat-diffusion-equation which leads to the non-trivial temperature and density profiles.

In our simulations the DPD thermostat is active all the time and removes the viscous heating almost instantaneously. Hence, our profiles $\Phi(y) / \Phi_{0}$ and $\left\langle m \mathbf{v}^{2}\right\rangle(y) / 3 k_{B} T$ in Fig. 4.11 are essentially flat. The spread around the mean values is of the order of $0.1 \%$ and two orders of magnitude smaller than in the cited work. The residual heating of $0.3 \%$ is a remainder of the viscous heating and vanishes if the driving is reduced even more.

The results of both methods, GK and RNEMD, offer consistent results in the range of $30-70 k_{B} T \times \tau / r_{c}^{2}$, where the RNEMD method provides one more significant digit than the GK method. Translating these numbers into physical units, one obtains $\eta=3.9 \times 10^{-10}$ Pams for lipid bilayers, $\bar{\rho}^{*}=18$, and $\eta=3.3 \times 10^{-8}$ Pams for polymeric bilayers, $\bar{\rho}^{*}=40$. The difference between lipid and polymer membranes is two orders of magnitude, but not a factor of 500 as found in experiments [292]. However, these two orders stem entirely from the conversion of units, which were obtained for rather generic systems. It might be possible that one gets a higher $\eta$ if specific systems are mapped. Nevertheless, $\eta$ matches the desired range of experimental values for lipid bilayers, $1-10 \times 10^{-10}$ Pams, exactly, so that the model provides a realistic view on the surface viscosity.

Tab. 4.1 displays the peculiar feature that $\eta$ decreases with increasing $\bar{\rho}^{*}$. This seems at first sight somewhat surprising, because the dynamic viscosity normally increases with the density. However, increasing the model parameter $\bar{\rho}^{*}$ does not only increase the number density of the lipids, but it also reduces the strength of the interactions (cf. Eq. (2.8)), so that the products $v_{A A} \times$ $\bar{\rho}^{*}, w_{A A A} \times\left(\bar{\rho}^{*}\right)^{2}, w_{A A B} \times\left(\bar{\rho}^{*}\right)^{2}$, and $w_{A B B} \times\left(\bar{\rho}^{*}\right)^{2}$ remain constant, but the actual coefficients vanish in the limit $\bar{\rho}^{*} \rightarrow \infty$. Only $v_{A B}$ and $v_{B B}$ keep finite values. In this limit the hydrophobic interior comprises only weakly interacting tails, and resembles the behavior of a polymeric melt of rather stiff, Gaussian chains [279]. The main contributions left to $\eta$ are in this case the intramolecular interactions. $^{12}$

\footnotetext{
${ }^{12}$ The intramolecular contribution should, however, follow the predictions of the Rouse model, where $\eta \propto \bar{\rho}^{*}$. Thus, it might be possible that $\eta$ develops a minimum at larger $\bar{\rho}^{*}$ and increases again afterwards.
} 


\section{Is it possible to vary $D$ and $\eta$ independently?}

The frictional forces of the ordinary DPD thermostat are controlled by the friction coefficient $\gamma$ and act only longitudinally. With this single parameter it is impossible to control several dynamical properties like the diffusion coefficient, $D$, and the viscosity, $\eta$, of a liquid independently.

In a recent article, Junghans et al. propose a modified DPD thermostat, the transverse DPD (TDPD) thermostat, that adds transverse stochastic forces with a second friction coefficient, $\gamma_{\perp}$ (cf. p. 29). They state that the "presented $[\ldots]$ thermostat can be used [...] to tune the diffusion constant and viscosity of the system to the desired values" [172]. If one assumes, that a change in $\gamma_{\perp}$ has only these two effects, i.e., it modifies $D$ and $\eta$, but it has a negligible influence on the hydrodynamic boundary conditions between the lipids, then this statement contradicts the Stokes-Einstein equation in two dimensions [316],

$$
D=k_{B} T / c_{2 D} \pi \eta .
$$

Here $c_{2 D}$ is a dimensionless constant, that depends only on these boundary conditions. In contrast to the well-known three-dimensional case, there is no dependency on the particle diameter in Eq. (4.63) because of the different units of $\eta$ in two dimensions. The problem with the cited statement is that according to Eq. (4.63) $D$ and $\eta$ are not independent, but their product is constant. In this small side project the question in the title shall be answered for our model. Thereby we check the validity of the two-dimensional Stokes-Einstein equation.

To this end several dedicated runs with $\bar{\rho}^{*}=18$ were started, that used the TDPD thermostat with $\gamma^{\|}=0.5 . \gamma^{\perp}$ was varied between 0 and 10 , the same range as in Ref. 172. $\eta$ was calculated by the GK formula, Eq. (4.29), and by the RNEMD method, Eq. (4.30). The molecular diffusion constant, D, was obtained by Eq. (4.1).

The top panel of Fig. 4.12 depicts $\eta$ obtained from the two methods as a function of $\gamma^{\perp}$. The GK formula underestimates $\eta$ and the discrepancy is getting worse with increasing $\gamma^{\perp}$. An increase of $\gamma^{\perp}$ leads to noisier data for $\left\langle P_{y z}(t) P_{y z}\right\rangle$, so that it becomes more and more difficult to obtain meaningful averages and to select an appropriate cutoff for the first plateau. It seems, that the integral over $\left\langle P_{y z}(t) P_{y z}\right\rangle$ was systematically truncated too early. However, there is hope, that much longer runs will yield better estimates for the cutoff time in the integration and therefore more accurate values of $\eta$.

As before, these difficulties are avoided in the RNEMD calculation, which produces an almost linear relationship. This linear relationship has also been observed by Junghans et al. in NEMD simulations using sliding boundary conditions [172]. In the following only the values of $\eta$ that are obtained from the RNEMD method will be discussed. 


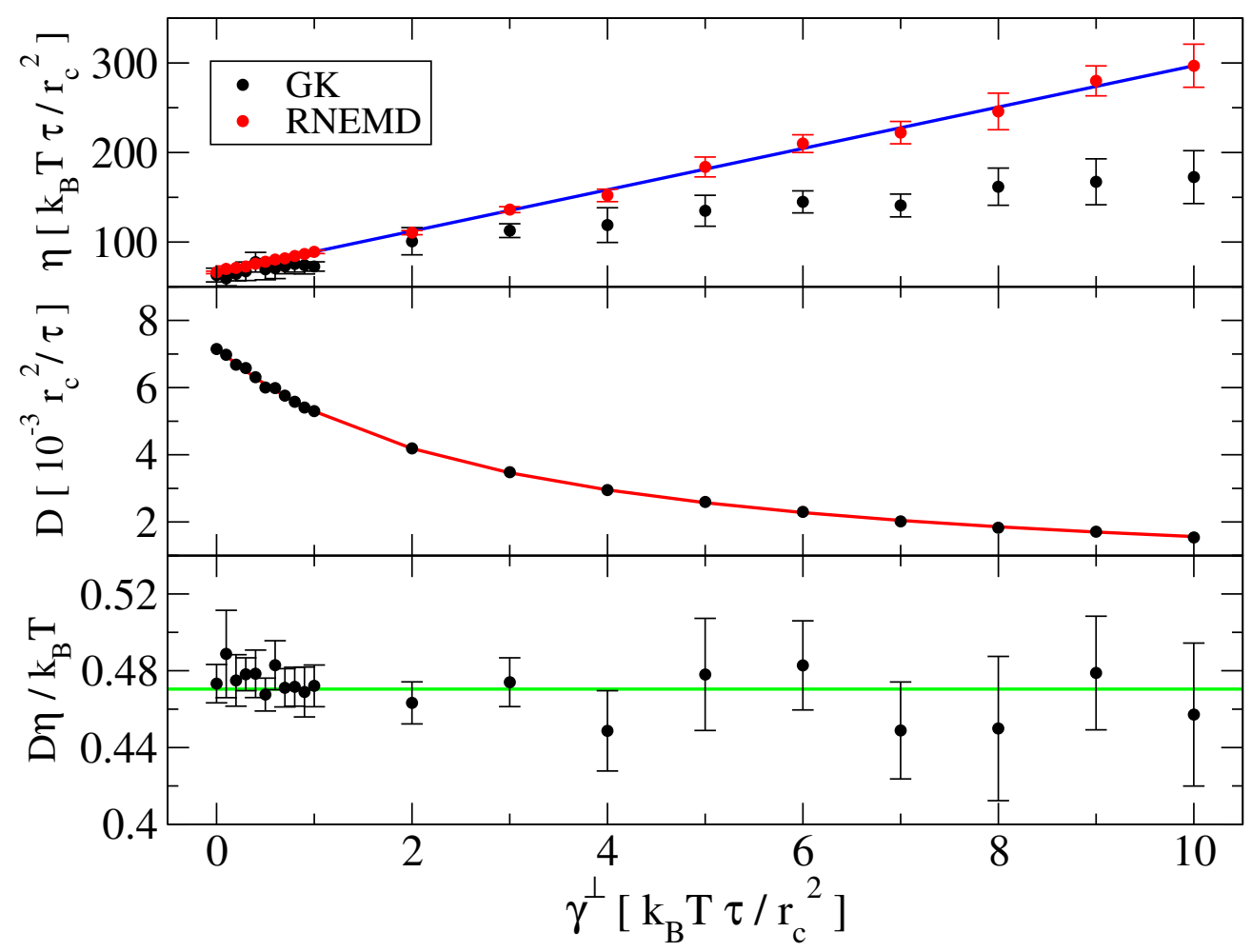

Figure 4.12: Top: surface viscosity $\eta$ for different values of the TDPD friction coefficient $\gamma^{\perp}$ measured by GK and RNEMD for the system $\bar{\rho}^{*}=18$. The blue line is a linear fit to the RNEMD data points. The GK method becomes less reliable for high $\gamma^{\perp}$, whereas the RNEMD method still works. Middle: molecular diffusion constant $D$ and a fit to the Einstein equation, Eq. (4.64). Bottom: according to Eq. (4.63) the product $D \eta / k_{B} T$ should be a constant and the green line depicts the best fit to this constant. 
The center panel of Fig. 4.12 presents $D$ as a function of $\gamma^{\perp}$. From the Einstein equation, $D \sim k_{B} T / \gamma$, one expects that $D$ and $\gamma^{\perp}$ are inversely related. Since the molecular diffusion coefficient is recorded and the standard DPD thermostat still contributes to the friction, we fitted the data to

$$
D=\frac{k_{B} T}{f\left(\gamma^{\perp}+\gamma_{0}\right)} .
$$

$f=49.9$ is a dimensionless scaling factor, whereas $\gamma_{0}=2.78 k_{B} T \times \tau / r_{c}^{2}$ contains the intrinsic friction of the standard DPD thermostat.

Finally, the product $D \eta / k_{B} T$ is depicted in the lower panel of Fig. 4.12. The error bars are obtained from the error propagation equation. Although they are large for $\gamma^{\perp}=4-10$, there is no trend in the data visible and the product seems to be constant within error bars. The existence of such a constant looks like a confirmation of the Stokes-Einstein equation, Eq. (4.63). At least for this specific set of interaction parameters, it is impossible to tune $D$ and $\eta$ independently. Finally, we obtain $c_{2 D}=0.15(1)$, which is much smaller than the value obtained in Ref. 316, $c_{2 D}=1.69(10)$, for a fluid of Yukawa discs. Unfortunately, no well-defined expressions relating $c_{2 D}$ to the type of the boundary condition exist, so that it is presented here only for the sake of completeness.

These findings indicate that an increase of $\gamma^{\perp}$ has hardly any effect on $D \eta / k_{B} T$. Although the TDPD thermostat successfully increases the numerical value of $\eta$, it also diminishes $D$ in the same way. Since the conversion of the simulation time to physical units is done by mapping the diffusion coefficients, $\eta$ stays constant in physical units. Hence, there is no additional flexibility in tuning the transport coefficients. The TDPD thermostat also increases the noise in the stress autocovariance, so that averaging becomes harder and the GK relations become de facto useless. Therefore, we conclude that the use of the TDPD thermostat offers no advantages in our model over the ordinary DPD thermostat.

\section{Measurement of the intermonolayer friction}

The intermonolayer friction, $b$, was obtained from the same equilibrium runs. Here, the key quantity is the instantaneous, total force on the upper leaflet, $\mathbf{F}^{+}$, exerted by the lower leaflet. $\mathbf{F}^{+}$was saved every $0.01 \tau$. In contrast to the GK measurement of $\eta$, two independent measurements of $b$ are this time available from a single run, because the two components $F_{y}^{+}$and $F_{z}^{+}$are uncorrelated. The recorded values of $F_{y / z}^{+}$were divided into non-overlapping blocks with $100 \tau$, the correlation function was calculated and integrated in each block, and averaged (see Fig. 4.13). The final $b$ is shown in Tab. 4.1 in 


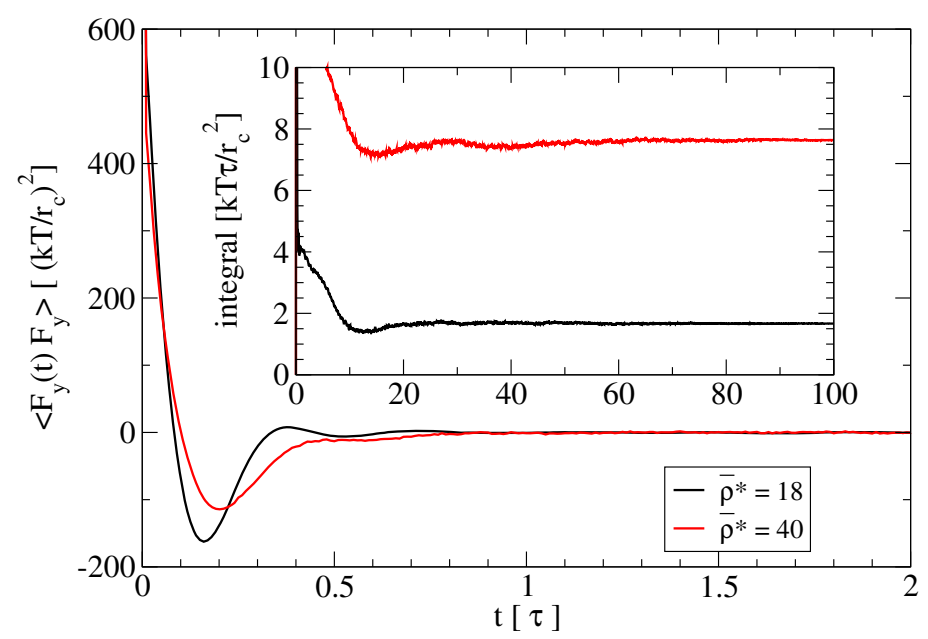

Figure 4.13: Autocovariance of the tangential force acting between the two monolayers (main panel) and its running integral (inset) for the two systems $\bar{\rho}^{*}=18$ and $\bar{\rho}^{*}=40$. $b$ is estimated by Eq. (4.36) from the integral in the limit $t \rightarrow \infty$. Therefore the running integral is shown for a longer time interval in the inset.

the column "Green-Kubo". The statistical uncertainty is obtained, as before, from a blocking analysis [315].

Fortunately, $\left\langle F_{\alpha}^{+}(t) F_{\alpha}^{+}\right\rangle$decays rapidly so that it is easy to compute accurate values of $b$ with Eq. (4.36). There are hardly any ambiguities regarding the position of the plateau in the integrated correlation function (see Fig. 4.13), thus, systematic uncertainties are small.

Finally, $b$ was computed from the autocorrelation function of the undulation modes, $C(q, t)=\left\langle h_{\mathbf{q}}(t) h_{\mathbf{q}}^{*}\right\rangle$. To this end $h_{\mathbf{q}}$ was calculated as on p. 46 and saved to disk every $0.02 \tau$. $C(q, t)$ was computed for the first 18 modes and averaged over all equivalent modes. ${ }^{13}$ Indeed, it shows some oscillations that vanish with increasing $q^{2}$ (see Fig. 4.14). In a next step $C(q, t)$ was Fourier transformed into the frequency domain, yielding the dynamic undulation structure factor, $S(q, \omega)$ (see Fig. 4.15). The fit to Eq. (4.62) for positive $\omega$ includes five fitting parameters, which all depend on $q: \Gamma_{R}, \Gamma_{B}, \omega_{B}, A_{R}$, and $A_{B}$.

Besides the dispersion relation of the propagating mode, $\omega_{B}(q)$ (see inset of Fig. 4.15), one also obtains the decay rates $\gamma_{1}(q)$ and $\gamma_{2}(q)$ (see Fig. 4.16). In principle the Eqs. (4.52-4.54) must be solved self-consistently for obtaining $\tau_{r}$ and $\omega_{h}$ from $\gamma_{1}$ and $\gamma_{2}$. However, we prefer Eq. (4.52) for evaluating $b$, because it depends only on $\gamma_{1}(q)$ and $\gamma_{2}(q)$. The other two equations, Eq. (4.53) and Eq. (4.54), depend additionally on $\omega_{B}(q)$ and $\tilde{\kappa} / \kappa$, or they contain $b$ only in higher order terms. $b$ 's statistical uncertainty was estimated by the error

\footnotetext{
${ }^{13}$ Two modes $h_{\mathbf{q}_{1}}$ and $h_{\mathbf{q}_{2}}$ are considered to be equivalent, if $\mathbf{q}_{1}^{2}=\mathbf{q}_{2}^{2}$.
} 


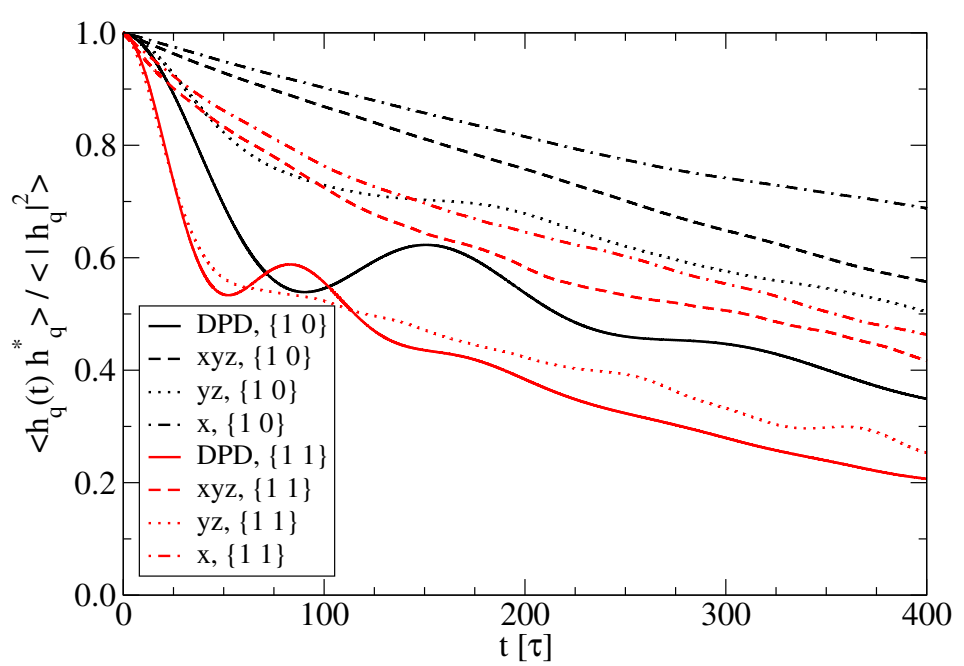

Figure 4.14: Autocorrelation function of the two slowest undulation modes, $\left\{\begin{array}{lll}1 & 0\end{array}\right\}$ (black) and $\left\{\begin{array}{ll}1 & 1\end{array}\right\}$ (red), averaged over all equivalent wave vectors for the system with $\bar{\rho}^{*}=18$. The solid line $(-)$ marks the result where the DPD thermostat was used, the dashed line (- -) marks the result where the Langevin thermostat was applied to all three directions, the dotted line $(\cdots)$ marks the result where it was applied to the two lateral directions, and the dash-dotted line (.-.) the result where it was applied only to the normal direction. The DPD result might be fitted with high accuracy to Eq. (4.61).

propagation equations, where it is implicitly assumed that $\gamma_{1}(q)$ and $\gamma_{2}(q)$ are uncorrelated. The most important contribution to the statistical uncertainty of $b$ stems from the errors in $\gamma_{1}$ and $\gamma_{2}$. The final results are listed in Tab. 4.1 in the column "Undulations".

An independent estimate of the order of magnitude of $b$ can also be obtained from theoretical considerations on polymer brushes [317, 318]. If one treats the two monolayers as apposing brushes that overlap in the center of the bilayer over a distance, $\delta$, and ignores all numerical prefactors, then $b \approx \rho_{c} \times \delta \times \xi$. Here, $\rho_{c}$ is the molecular number density and $\xi=c_{2 D} \times \eta$ is a friction coefficient. $\delta$ is readily obtained from the width of $\hat{\rho}_{A}^{+}(x) \times \hat{\rho}_{A}^{-}(x)$, where $\hat{\rho}_{A}^{ \pm}(x)$ is the density profile of the hydrophobic beads on the upper/lower leaflet [317], cf. Fig. 3.6. Using $\delta=0.71 r_{c}, c_{2 D}=0.15(1), \eta=66.2(7) k_{B} T \tau / r_{c}^{2}$, we find

$$
b \approx 3 k_{B} T \times \tau \times r_{c}^{-4}
$$

for $\bar{\rho}^{*}=18$ which gives the right order of magnitude.

A comparison of the three methods shows that they provide consistent results, although those of the GK formula are more significant by one digit. This is largely because the GK formula is a much more direct method of probing $b$. 


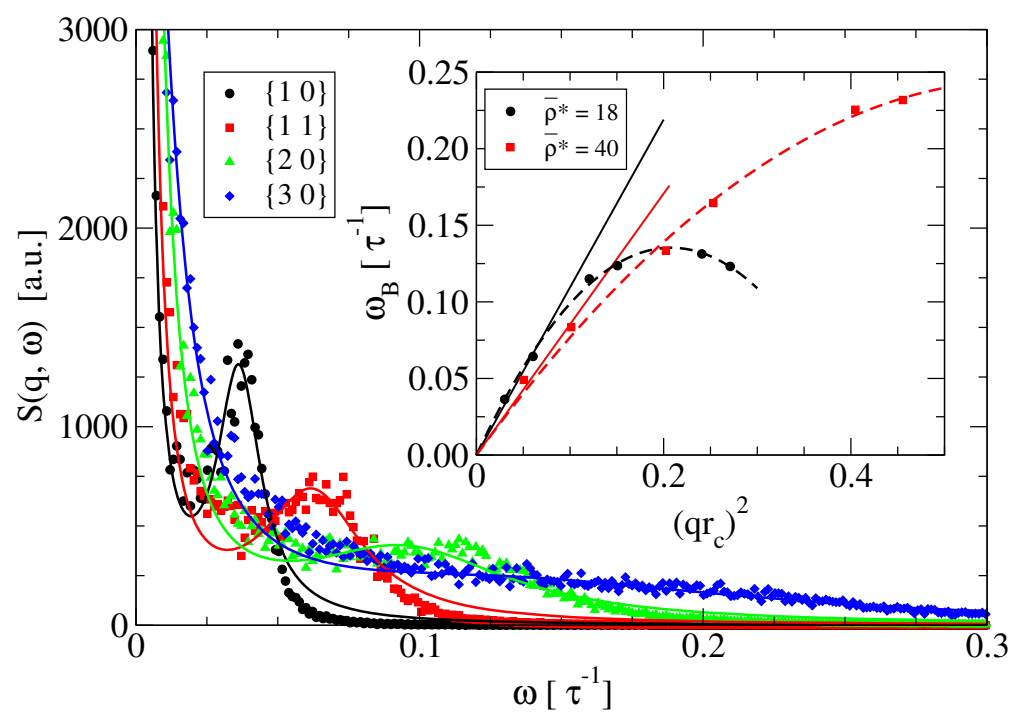

Figure 4.15: Main panel: dynamic undulation structure factor $S(q, \omega)$ for four undulation modes of the system $\bar{\rho}^{*}=18$, showing the Rayleigh line at $\omega=0$ and the Brillouin line at $\omega=\omega_{B}$. The solid lines are fits to Eq. (4.62). Inset: dispersion relation, $\omega_{B}(q)$, of the propagating mode for $\bar{\rho}^{*}=18$ and $\bar{\rho}^{*}=40$. The solid lines are fits to the asymptotical $\omega_{B} \sim q^{2}$ power law, whereas the dashed lines include a higher order term $\mathcal{O}\left(q^{4}\right)$.

Qualitatively, the same difference in the accuracy also showed up, when $b$ was compared by other authors between NEMD simulations with Lees-Edwards sliding boundary conditions and the Seifert-Langer theory [304]. In that case the NEMD results are easier to obtain and have a higher accuracy.

Translating the values from Tab. 4.1 for $\bar{\rho}^{*}=18$ to physical units we obtain $b=1.6 \times 10^{6} \mathrm{Pas} / \mathrm{m}$. Compared to the experimental range of $b=$ $1-10 \times 10^{8} \mathrm{Pas} / \mathrm{m}$ for lipid bilayers, our result is approximately two orders of magnitude too small. However, it is close to the two other simulation results we are aware of. Den Otter and Shkulipa simulate a coarse-grained model of 1518 DPPC lipids in an explicit solvent and find, depending on the details of the coarse-grained representation of a lipid, $b=2.4-7.3 \times 10^{6} \mathrm{Pas} / \mathrm{m}$ [304]. Wohlert and Edholm obtain $b=2.8 \times 10^{6} \mathrm{Pas} / \mathrm{m}$ in an atomistic study of 128 DMPC molecules surrounded by water [278]. Although these three results have been obtained by different techniques and from completely different models, the order of magnitude of $b$ seems to be universal for lipid bilayers. Hence, we agree with the other two studies claiming that there might be a problem with the interpretation of the experimental results.

The same problem might also arise in polymeric membranes. Here we find $b=8.7 \times 10^{8} \mathrm{Pas} / \mathrm{m}$ for $\bar{\rho}^{*}=40$. This value is also $1-2$ orders of magni- 


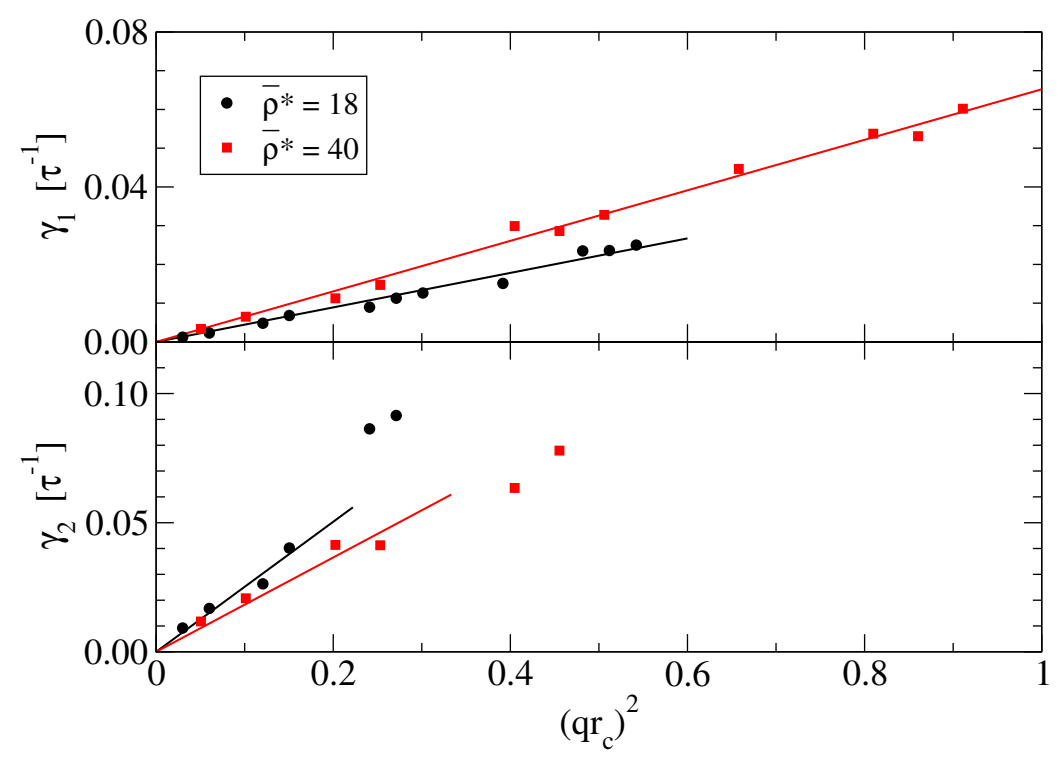

Figure 4.16: Top: the decay rate, $\gamma_{1}$, of the undulation modes as a function of $q^{2}$ for $\bar{\rho}^{*}=18$ and $\bar{\rho}^{*}=40$. The solid lines are fits to all data points. Bottom: decay rate $\gamma_{2}$ of the propagating undulation mode. The solid lines are fits to the first four data points.

tude smaller than the reported experimental values [292]. To the best of our knowledge nobody has measured $b$ at the time of this writing for such a system. It remains an open question, whether there is a general problem with the interpretation of all experiments measuring the intermonolayer friction.

\section{Origin of the oscillations}

How can one be sure, that the oscillations of the undulation autocorrelation in Fig. 4.14 are really caused by inertia, and not by some other effect? ${ }^{14}$. In the following, a series of consistency checks is discussed.

The first and simplest check is to disable the conservation of momentum temporarily. Of course, such a check is only possible in a computer simulation, but here it can be achieved by switching from the DPD thermostat to the Langevin thermostat which violates momentum conservation (cf. p. 29). For keeping the temperature constant, it is sufficient to couple the thermostat to only a subset of the translational degrees of freedom, for instance to all $y$ coordinates. This is interesting, because it leads to a violation of momentum conservation in only this direction, while the momentum is still conserved in the other directions.

\footnotetext{
${ }^{14}$ Seifert's original work includes several extensions which all lead to propagating modes [84]
} 
Here, three cases are discussed, where the Langevin thermostat is coupled to (i) all degrees of freedom of the beads, (ii) only the lateral ones, and (iii) only the normal ones. The difference in Fig. 4.14 is striking: in case (i), the conservation of momentum is violated in all directions and consequently there are no oscillations visible. The autocorrelation function shows an exponential decay, which is probably the case one would observe in a standard MC simulation, or in the presence of a solvent. In case (ii), the momentum is conserved only in the normal direction, and is violated laterally. This situation is similar to what one expects in DPD simulations, because the two-dimensional liquid of lipids is overdamped and inertia effects can be neglected. Indeed, oscillations with a comparable frequency in the autocorrelation function are observed. They are, however, less pronounced than in the case of DPD, because the bilayer is not a true two-dimensional liquid. The application of the thermostat in case (iii) completely destroys all inertia effects in the normal direction and leads to a low damping of the undulatory modes and therefore to the slowest decay. Consequently, there are no oscillations visible.

The inertia effect and its analytical treatment can also be checked for consistency by inspecting the dependency of $\gamma_{1}(q), \gamma_{2}(q)$, and $\omega_{B}(q)$ on $q$ in the lowest order. The inset of Fig. 4.15 depicts $\omega_{B}(q)$ for $\bar{\rho}^{*}=18$ and $\bar{\rho}^{*}=40$. A fit to the first two data points shows a dependency $\omega_{B} \sim q^{2}$, which is consistent with Eq. (4.60). For larger $q^{2}$ deviations show up, that can still be fitted by including a second order term in $q^{2}$, i.e., $\mathcal{O}\left(q^{4}\right)$. In principle it is possible to extract the bending rigidity, $\kappa$, from the slope at small $q$. We obtain $\kappa=7$, and $\kappa=11$ for $\bar{\rho}^{*}=18$ and 40 , respectively. The moderate agreement with the measurement in Sec. 3.1.4 is not too surprising, since the higher order terms are important and only two data points were used for the fit. The actual slope of $\omega_{B}\left(q^{2}\right)$ should therefore be roughly a factor 1.4 higher than calculated here.

Figure 4.16 displays $\gamma_{1}$ and $\gamma_{2}$ as functions of $q^{2}$ which are directly related to the widths of the Rayleigh and the Brillouin lines. $\gamma_{2}(q)$ has only a limited number of data points, because it becomes increasingly difficult to track the width and position of the Brillouin line with increasing $q$. We deduce from the linear relationship that both depend in lowest order on $q^{2}$, as it is expected from Eq. (4.58) and Eq. (4.59). Notably, $\gamma_{2} \approx 0.4 \times \gamma_{1}$, for both systems, which supports the idea that the leading term in $\gamma_{2}$ is $\mathcal{O}(\varepsilon)$ (cf. Eq. (4.56)).

The origin of the oscillation is entirely different from the oscillation that is observed in standard hydrodynamic coupling theory [83, 192, 319-321], which takes only the membrane tension and the solvent viscosity into account. ${ }^{15}$ In this theory a dispersion $\omega \sim q^{3 / 2}$ for propagating, transverse capillary waves is found, that has no additional peak at $\omega=0$ in the dynamic structure factor $S(q, \omega)$.

\footnotetext{
${ }^{15}$ This theory is only valid for wavelengths that are much larger than the membrane thickness, which is probably not the case in this work
} 
As a side remark: a simple change of the mass, $m \rightarrow x \times m$, of all lipids disqualifies itself for being a check of the model. Although $m$ enters in $\gamma_{1}, \gamma_{2}$, and $\omega_{B}$, a change leads only to a trivial rescaling of the time scale, i.e., $\tau \rightarrow$ $\sqrt{x} \tau$. Hence, no new insight into the validity of the model is gained.

After this discussion it is apparent how to get an undulation mode's autocorrelation which resembles the experimental result: the bilayer must be able to exchange momentum with its surroundings. ${ }^{16}$ This can be either achieved by introducing a solvent into the model, or by coupling a small fraction of the degrees of freedom to a thermostat that does not conserve momentum. A possibility could be to couple only the head groups to a Langevin thermostat, which mimics the momentum that would normally be transferred to the solvent.

\subsubsection{Conclusions}

In a first step a monolayer's surface viscosity, $\eta$, was measured by two different methods. The first one is based on a GK formula and uses equilibrium fluctuations to extract $\eta$ from the autocorrelation of the off-diagonal elements of the pressure tensor. In the second method, RNEMD, an artificial flux of lipids is created, and the corresponding velocity profile is measured which can be related to $\eta$. The results of both methods agree within error bars, however, the results of the RNEMD measurement provide one order of magnitude better accuracy at comparable compute time than the GK measurements, which are plagued by the problem of finding the "right" plateau. Standard NEMD measurements with sliding boundary conditions could not be applied, because of the lack of solvent. The values for $\eta$ are in the range of $30-70 k_{B} T \times \tau / r_{c}^{2}$. Converting these numbers into physical units, we obtain $\eta=3.9 \times 10^{-10} \mathrm{~Pa} \mathrm{~m} \mathrm{~s}$ for $\bar{\rho}^{*}=18$ and $\eta=3.3 \times 10^{-8}$ Pams for $\bar{\rho}^{*}=40$. Both numbers match the experimental range and the resulting surface viscosity of polymer membranes is approximately two orders of magnitude higher than that of lipid bilayers.

In a second step the intermonolayer friction coefficient, $b$, was measured by two methods that have not been used before to measure $b$. The first one is based on another GK relation that relates the integral of the autocovariance of the total lateral force between the monolayers to $b$. In contrast to the GK relation for $\eta$, this one yields results with high accuracy, because the autocovariance decays rapidly. The GK formula is known from fluid mechanics, where it is used to extract friction coefficients of rough surfaces [313, 314].

${ }^{16}$ There is of course the possibility of a negative discriminant of Eq. (4.49), so that three real eigenvalues come into existence. The detailed conditions can be formulated by two inequalities, but this has not been worked out. It seems necessary for $\gamma_{1}$, which is a function of $k_{m}$, to become very large; in this case $k_{m}$ has to acquire unphysically high values. 
The second method is based on a theory by Seifert and Langer, which incorporates $b$ into the decay rate of the undulation mode autocorrelation [84, 85]. This method is a very indirect one, and therefore depends on the prior measurement of several other quantities, like the bending rigidity and the monolayer compression modulus. Unfortunately, the original theory cannot be applied to a solvent-free model, because inertia effects come into play which are not treated. These effects change the behavior of the undulation autocorrelations qualitatively and lead to an oscillatory decay. An improved theory without any free parameters has been derived that explains these oscillations. Several consistency checks have been performed, so that we are confident about the origin of these oscillations. The results of both methods agree within error bars, although the second one is rather inaccurate. The obtained values for $b$ are in the range of $1-8 k_{B} T \times \tau / r_{c}^{4}$, which translates to $b=1.6 \times 10^{6} \mathrm{Pas} / \mathrm{m}$ for $\bar{\rho}^{*}=18$ and $b=8.7 \times 10^{8} \mathrm{Pas} / \mathrm{m}$ for $\bar{\rho}^{*}=40$. Compared to the experiments, both values are 1-2 orders of magnitude too small, but they are consistent with other coarse-grained and atomistic simulations. We therefore conclude that there might be a general problem with the interpretation of the experiments.

In summary the model reproduces realistic values for $\eta$. If the DPD thermostat is used and no solvent is present, then the decay of the undulations is different from what is observed in experiments. This artifact can be circumvented by a partial violation of the conservation of momentum, or by the introduction of a solvent. Moreover, we have explored the possibility of using the TDPD thermostat to control the diffusivity and the viscosity independently. It turned out that the product $D \times \eta$ is constant within error bars. This finding is consistent with the two-dimensional Stokes-Einstein equation.

\subsection{Mapping to a fluid of soft discs}

Our coarse-grained bead-spring model, presented so far, exhibits many static and dynamic properties of amphiphilic bilayers that are compatible with experimental observations. Even though the number of degrees of freedom has been reduced significantly compared to atomistic models, its applicability is still limited to situations whose characteristic dimensions are smaller than $100 \mathrm{~nm}$ in the spatial and $10 \mu \mathrm{s}$ in the time domain. For many applications in chemical biology this is still not enough, therefore even coarser coarse-grained models become necessary.

Under certain circumstances, it is of interest to study the interplay of phenomena on separated time or length scales. In these cases one requires differently coarse-grained representations of the same physical systems which must be connected somehow. For instance, one could envisage the study of vesicles in shear flow, which is a simplified model for the flow of red blood cells in blood 

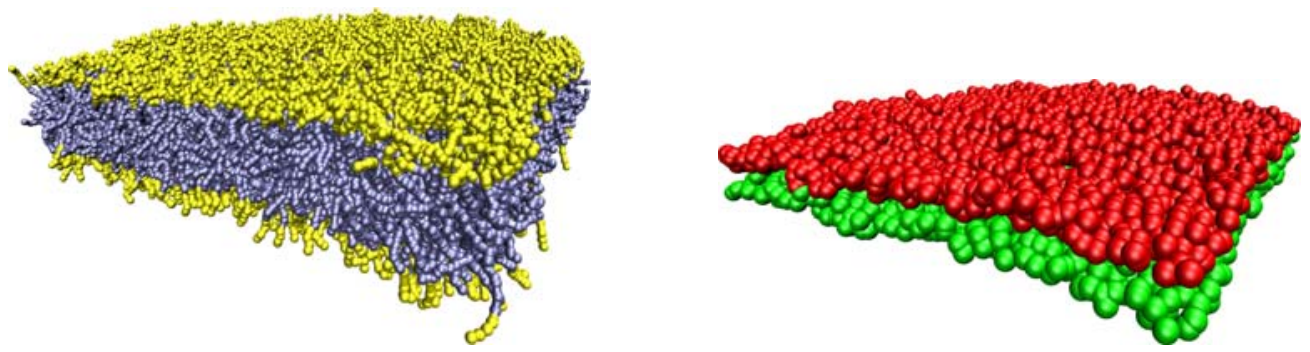

Figure 4.17: In this section the lipids in the HRM (left) are mapped to a fluid of soft, two-dimensional discs, the LRM. To give theses discs a spatial extension they are drawn as spheres instead (right).

vessels. Ideally, all three dissipation mechanisms, i.e., the solvent viscosity, the intermonolayer friction, and the surface viscosity, would be included. However, the latter ones can be only accounted for if the molecular structure of the bilayer is considered. At the moment such a study is still unfeasible and other authors resorted to models without such detail; for instance they described the bilayer surface by a triangulation [106, 107, 322].

In recent years, sophisticated "multi scale" coarse-graining methodologies have been invented for tackling such problems. Typically they encompass two or more representations of the same system on different levels of coarsegraining. In the case of only two representations there is normally one with a high resolution, i.e., an atomistic or molecular representation, and one with a low resolution which is more coarse-grained. In the following the former one will be denoted as the high resolution model (HRM) and the latter one as the low resolution model (LRM). Generally, a successful connection between these two representations involves three fundamental steps [100].

The first step is to define a prescription for mapping the HRM's "atoms" to some effective interaction centers in the LRM, e.g., by lumping several atoms into one bead. If dynamic properties are of interest, the momenta must be mapped additionally. In the following we will map an entire amphiphilic molecule onto a single bead sitting at the molecule's center of mass (see Fig. 4.17). The bead's momentum is given by the molecule's center of mass momentum.

The second step consists of mapping the interactions of the HRM's atoms to the LRM's beads. Various schemes have been presented for finding these interactions and many of them are able to reproduce the HRM's statics with high accuracy. Most prominent are iterative schemes that reproduce a set of lower order radial distribution functions, like inverse Monte Carlo [323-325], the iterative Boltzmann inversion method [96, 326, 327], the structure based coarse-graining [328, 329], or the molecular renormalization group approach 
[330, 331]. They have been applied successfully in several studies of lipid bilayer membranes [325, 332, 333]. A different, non-iterative scheme is based on solving the Born-Green-Yvon (BGY) set of integral equations known from liquid-state theory numerically [334, 335]. In this way variationally optimized interaction potentials can be generated from existing structural correlation functions.

Finally, there is the "force-matching" scheme, where arbitrary many-body interaction potentials are cast into a set of pair potentials that are expanded as linear combinations of suitable basis functions [98-101]. The coefficients of this expansion are determined variationally, by minimizing the discrepancy between the forces of the HRM and the matched forces of the LRM. Several successful applications of this scheme in lipid bilayer studies have been reported [67, 98, 336-338].

Reproducing only static properties is not enough for preserving the dynamics of the underlying model. ${ }^{17}$ In fact, the coarse-graining eliminates many microscopic degrees of freedom which act as a heat bath and cause thermal noise and friction for the macroscopic degrees of freedom. Therefore, a third step is needed, in which the HRM's friction is imprinted on the LRM. This step has been overlooked in many studies so far, and work on this topic has begun only recently [86-88]. At present no practical, working schemes are available and the mapping of friction is largely based on physical intuition.

An easy way of reintroducing friction and thermal noise into the LRM is to let an adjusted DPD thermostat do the work. This marks a change in the perception of the thermostat: instead of being responsible for maintaining only the temperature, its stochastic forces now replace the microscopic background that has been eliminated [141]. By manipulating the thermostat's parameters, i.e., the friction coefficient, the weighting function, or the cutoff, it is possible to control the friction [172, 339-341] and it might be possible to adjust the LRM's transport coefficients to the HRM's values.

In this section we use our coarse-grained model as the HRM and perform an additional coarse-graining step for obtaining a simplified representation. The lipids in this LRM are represented by two-dimensional soft discs interacting via pair potentials (see Fig. 4.17). The goal of this study is to establish a representation of our model with the same pair correlations on large and small scales, and the same dissipation coefficients, i.e., the intermonolayer friction and the surface viscosity. The consideration of the dissipation marks an improvement over previous work, where only static properties have been matched [332, 338]. This toy model allows us to perform very efficient simulations and to gain insight into the relevance of the degrees of freedom for the dynamics, as well as to study the influence of the DPD thermostat and its parameters on the transport coefficients.

\footnotetext{
${ }^{17}$ It has been pointed out that reproducing only static properties is not even enough for preserving the thermodynamics [77].
} 


\subsubsection{Interactions}

The mapping of the HRM to the LRM is achieved by the three steps mentioned before. First, a simple, two-dimensional mapping prescription is established, where the lipids are represented by a single bead which resides at the lateral position of its center of mass. The normal coordinate is discarded. Second, the interaction potentials of the LRM are engineered to approximate the lateral pair correlation functions and the static structure factor of the HRM; this is achieved by a force-matching procedure. Third, the intermonolayer friction and the surface viscosity of the LRM particles are adjusted to the HRM's values by tuning the DPD thermostat's parameters.

\section{Force-matching}

The $n$ lipids in the LRM are structureless, two-dimensional point particles confined to a plane. To mimic the existence of two leaflets, half of the lipids are tagged as residing on the lower leaflet, and half of them as residing on the upper one. All lipids interact through two pair potentials: an intraleaflet potential, $U_{\text {intra }}(r)$, for the interactions between two lipids within the same leaflet, and an interleaflet potential, $U_{\text {inter }}(r)$, for the interactions between lipids on opposite leaflets. In the following $r$ denotes the lateral distance between two beads, irrespective of their leaflets.

The interactions of the HRM are mapped to $U_{\text {intra }}(r)$ and $U_{\text {inter }}(r)$ by a force-matching procedure. An extensive discussion of this procedure is given elsewhere [98-101], thus only a brief description of the relevant issues for this work is given. The presentation follows Ref. 101.

The matching prescription that defines the LRM's positions, $\mathbf{R}_{I}$, and momenta, $\mathbf{P}_{I}$, as functions of the HRM's positions, $\mathbf{r}_{I i}$, and momenta, $\mathbf{p}_{I i}$ is:

$$
\begin{aligned}
& \mathbf{R}_{I}=\frac{1}{N} \sum_{i=1}^{N}\left(\hat{\mathbf{e}}_{y} \otimes \hat{\mathbf{e}}_{y}+\hat{\mathbf{e}}_{z} \otimes \hat{\mathbf{e}}_{z}\right) \cdot \mathbf{r}_{I i} \\
& \mathbf{P}_{I}=\frac{1}{N} \sum_{i=1}^{N}\left(\hat{\mathbf{e}}_{y} \otimes \hat{\mathbf{e}}_{y}+\hat{\mathbf{e}}_{z} \otimes \hat{\mathbf{e}}_{z}\right) \cdot \mathbf{p}_{I i}
\end{aligned}
$$

The two indices of the HRM quantities are the molecular index, $I$, and the bead index, $i$, of molecule $I$. The quantities of the LRM are written in upper case, whereas those of the HRM are written in lower case. According to the classification of Noid et al. [100], this mapping is "consistent in phase space", i.e., the LRM's "equilibrium distribution of coordinates and momenta is equal to the distribution determined by the [HRM's] equilibrium distribution function". An important feature of this mapping is the equivalence of the unit of length, $r_{c}$, in both the LRM and the HRM. 
The LRM's total potential energy can be written as

$$
U\left(\mathbf{R}^{n}\right)=\sum_{x, \gamma} U_{x}\left(r\left(\{\mathbf{R}\}_{\gamma}\right)\right)
$$

where $n$ denotes the number of molecules, $x \in\{$ intra, inter $\}$ one of the two possible interactions, and $\gamma$ a specific pair of LRM particles that has an interaction of type $x$. The function $r\left(\{\mathbf{R}\}_{\gamma}\right)$ gives the lateral distance between the two particles. Both potentials, $U_{x}(r)$, have a finite range and vanish for $r \geq \Lambda$. They are gauged so that $U_{x}(\Lambda)=0$. In the interval $[0, \Lambda]$ they are constructed from a set of $K$ basis functions, $u_{x d}(r)$, and are written as linear combinations with real coefficients, $\phi_{x d}$, as follows

$$
U_{x}(r)=\sum_{d=1}^{K} \phi_{x d} u_{x d}(r) .
$$

The corresponding force on particle $I$ is therefore

$$
\mathbf{F}_{I}\left(\mathbf{R}^{n} ; \phi\right)=\sum_{x, d} \phi_{x d} \mathbf{G}_{I ; x d}\left(\mathbf{R}^{n}\right)
$$

with

$$
\mathbf{G}_{I ; x d}\left(\mathbf{R}^{n}\right)=-\sum_{\gamma} \frac{d u_{x d}\left(r\left(\{\mathbf{R}\}_{\gamma}\right)\right)}{d r} \frac{\partial r\left(\{\mathbf{R}\}_{\gamma}\right)}{\partial \mathbf{R}_{I}} .
$$

Equation (4.70) demonstrates that $\mathbf{F}_{I}$ is linear in the expansion coefficients $\phi_{x d}$. The force-matching has the consequence that the unit of energy, $k_{B} T$, agrees in the two models in addition to $r_{c}$.

In this work we use fourth order B-splines for the basis functions, $u_{x d}(r)$, i.e., cubic polynomials which are defined in a compact interval and are continuously differentiable at the interval boundaries. They are widely used in many different areas where fitting problems occur $[336,342]$. It can be shown easily that the gauge implies $\phi_{x K}=0$.

As it was shown by Noid et al. the force-matching procedure can be formulated as a variational principle in the coefficients $\phi_{x d}$ that is very similar to the one used in condensed matter physics to determine ground-state wave functions $[100,101]$. In our two-dimensional case, the functional to be minimized is

$$
\chi_{\mathrm{MS}}^{2}(\phi)=\frac{1}{2 n}\left\langle\sum_{I=1}^{n}\left\|\mathbf{f}_{I}\left(\mathbf{r}^{n N}\right)-\mathbf{F}_{I}\left(\mathbf{R}^{n} ; \phi\right)\right\|^{2}\right\rangle .
$$

Here, $\mathbf{f}_{I}\left(\mathbf{r}^{n N}\right)$ is the total lateral force on the center of mass of lipid $I$ according to the interactions of the HRM. In general, this depends on the positions of all $n N$ beads, $\mathbf{r}^{n N}$, in the HRM. The positions of the LRM particles, $\mathbf{R}^{n}$, are also 
functions of $\mathbf{r}^{n N}$ with the relationship being given by the mapping operator. For obtaining an expression that is easier to handle, we rewrite Eq. (4.72) as a time average over $N_{t}$ sampled time steps. By using Eq. (4.70) we find

$$
\begin{aligned}
\chi_{\mathrm{MS}}^{2}(\phi) & \simeq \frac{1}{2 N_{t} n} \sum_{t=1}^{N_{t}} \sum_{I=1}^{n}\left\|\mathbf{f}_{I}\left(\mathbf{r}_{t}^{n N}\right)-\sum_{x d} \phi_{x d} \mathbf{G}_{I ; x d}\left(\mathbf{R}^{n}\right)\right\|^{2} \\
& =\frac{1}{2 N_{t} n}\|\underline{\mathbf{f}}-\underline{\underline{\mathbf{G}}} \underline{\phi}\|^{2} .
\end{aligned}
$$

In the second line, $\underline{\mathbf{f}}$ denotes the vector of $2 N_{t} n$ force components, $\phi$ the vector of $2 K$ expansion coefficients, and $\underline{\underline{\mathbf{G}}}$ the $2 N_{t} n \times 2 K$ matrix of all force components; $\|\cdot\|$ is the usual Euclidean norm.

From a technical point of view, Eq. (4.73) depicts a linear least-squares problem, ${ }^{18}$ with $2 K$ parameters $\phi_{x d}$, that can be readily solved with one of the many numerical libraries. ${ }^{19}$ Since $\underline{\underline{\mathbf{G}}}$ gets very large for large $N_{t}$, it is virtually impossible to keep all sampled time steps in the computer's memory at the same time. Therefore the block averaging approximation is used [101]. In this approximation the $N_{t}$ samples are grouped into $m$ equal blocks of length $\tilde{N}_{t}=N_{t} / m$. One computes an estimate $\underline{\phi}_{i}, i=1 \ldots m$, from each block $i$, and finally yields $\underline{\phi}=\sum_{i} \underline{\phi}_{i} / m$. Besides the reduced memory consumption, this approximation offers the benefit of speeding up the calculation of $\phi$, because the computational cost of the least-squares problem scales with the block length like $\mathcal{O}\left(\tilde{N}_{t}^{2}\right)$.

\section{Friction-matching}

The projection onto the centers of mass removes all intramolecular degrees of freedom, so that the intrinsic friction of the LRM is expected to be much smaller than that of the HRM. Hence, the LRM's $b$ and $\eta$ will differ from the HRM's values. Of course, the unit of time in the LRM does not have to agree with the one in the HRM, thus, a new mapping of the time scale, similar to p. 90, becomes necessary.

Instead of performing the additional mapping explicitly, we focus in this section on the time scale independent products $D b / k_{B} T$ and $D \eta / k_{B} T$. The first one has the dimension of an inverse area, while the latter one is dimensionless. To distinguish the HRM's target quantities from the LRM's actual quantities,

\footnotetext{
${ }^{18} \mathrm{In}$ fact it is a constrained least-squares problem, because Eq. (4.73) is accompanied by two linear constraints, $\phi_{x K}=0$, which result from the potential gauge, $U_{x}(\Lambda)=0$. Since the constraints are linear, the constrained least-squares problem with $2 K$ degrees of freedom can be rewritten as an unconstrained linear least-squares problem with $2(K-1)$ degrees of freedom.

${ }^{19}$ Here the function dgglse() of the LAPACK library is used.
} 
we denote the latter ones with a tilde, e.g., $\tilde{b}, \tilde{\eta}$, and $\tilde{D}$. Since the units of length $\left(r_{c}\right)$ and energy $\left(k_{B} T\right)$ agree in both models, the equations $\tilde{D} \tilde{b} / k_{B} T \approx D b / k_{B} T$ and $\tilde{D} \tilde{\eta} / k_{B} T \approx D \eta / k_{B} T$ have to hold for the mapping to be successful.

For adjusting the friction of the LRM we have to introduce a new source of friction, which will be the DPD thermostat. We have learnt in Sec. 4.2 that two different dissipation mechanisms exist and, hence, we will use a variant of this thermostat that distinguishes the interactions within the same leaflet from those between opposite leaflets. Specifically, we assign an intraleaflet friction coefficient, $\gamma_{\text {intra }}$, and an intraleaflet (DPD-)weighting function, $w_{\text {intra }}^{D}(r)$, to the

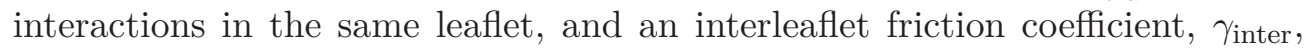
and a corresponding weighting function, $w_{\text {inter }}^{D}(r)$, to the other case (cf. the discussion of the standard DPD thermostat on p. 28). Hence, we have two parameters $\left(\gamma_{\text {intra }}, \gamma_{\text {inter }}\right)$ and two functions $\left(w_{\text {intra }}^{D}(r), w_{\text {inter }}^{D}(r)\right)$ as degrees of freedom for tuning the LRM's dynamics. A proper shape of the weighting functions has to be determined.

For saving computing time, one would like to have an analytic expression that relates the four degrees of freedom of the DPD thermostat $\left(w_{\text {intra }}^{D}(r), w_{\text {inter }}^{D}(r)\right.$, $\left.\gamma_{\text {intra }}, \gamma_{\text {inter }}\right)$ to $\tilde{D}$. It has been shown by Lahmar and Rousseau that such an expression approximately exists [341]. Here, we extend this expression to the case of two coupled, two-dimensional monolayers. For brevity we introduce the notation

$$
[f] \equiv 2 \pi \Phi_{0} \int_{0}^{\Lambda} r d r f(r)
$$

to denote an areal integral of an arbitrary function $f(r)$ over the disc with radius $\Lambda ; \Phi_{0}=n / A$ is the lipid number density. By considering the lateral pair correlation function of beads in the same leaflet, $g_{\text {intra }}(r)$, and in different leaflets, $g_{\text {inter }}(r)$, we define effective friction coefficients as

$$
\begin{aligned}
& \bar{\gamma}_{\text {inter }}=\gamma_{\text {inter }}\left[g_{\text {inter }} w_{\text {inter }}\right] \\
& \bar{\gamma}_{\text {intra }}=\gamma_{\text {intra }}\left[g_{\text {intra }} w_{\text {intra }}\right]
\end{aligned}
$$

These integrals project the four degrees of freedom onto two scalar quantities. For physical reasons, $\bar{\gamma}_{\text {inter }}$ and $\bar{\gamma}_{\text {intra }}$ are always positive. Due to the softness of our interactions, packing effects are negligible, i.e., $g_{\text {intra }}(r) \approx g_{\text {inter }}(r) \approx 1$. This fact can be used to simplify these expressions to ${ }^{20}$

$$
\bar{\gamma}_{\text {inter }} \approx \gamma_{\text {inter }}\left[w_{\text {inter }}\right] \text { and } \bar{\gamma}_{\text {intra }} \approx \gamma_{\text {intra }}\left[w_{\text {intra }}\right]
$$

The lateral diffusion coefficient may now be cast approximately in the form of

\footnotetext{
${ }^{20}$ Keeping $g_{\text {intra }}(r)$ and $g_{\text {inter }}(r)$ in the expressions changes the numerical values of $\bar{\gamma}_{\text {intra }}$ and $\bar{\gamma}_{\text {inter }}$ by less than $5 \%$.
} 
a Stokes-Einstein equation as [341]

$$
\tilde{D}=c_{1} \times \frac{k_{B} T}{\gamma_{0}+\left(\bar{\gamma}_{\text {intra }}+\bar{\gamma}_{\text {inter }}\right)},
$$

where $c_{1}$ is a positive, dimensionless coupling constant and $\gamma_{0}$ is the (small) LRM's intrinsic friction originating from the interaction potentials. Equation (4.78) depends only on the sum of the two effective friction coefficients. Hence, this universality allows us to predict the value of $\tilde{D}$ for all DPD parameters, once the constants $c_{1}$ and $\gamma_{0}$ are fixed.

It is tempting to speculate if a similar expression also holds for the intermonolayer friction coefficient, $\tilde{b}$. However, such a relationship has not yet been found. Here, we propose a linear relationship involving only $\bar{\gamma}_{\text {inter }}$, because this is the essential source of the friction between the leaflets. For obtaining the right units, $\tilde{b}$ should also be proportional to $\Phi_{0}$. Hence, ${ }^{21}$

$$
\tilde{b}=c_{2} \Phi_{0} \times \bar{\gamma}_{\text {inter }},
$$

where $c_{2}$ is another dimensionless coupling constant, independent of the thermostat. This expression should be valid for intermediate friction, but will fail if $\bar{\gamma}_{\text {inter }}$ approaches zero, since the intrinsic intermonolayer friction is neglected in Eq. (4.79). At very high friction, non-linear terms, which are currently neglected, can become relevant.

Multiplying $\tilde{D}$ and $\tilde{b}$, i.e., Eq. (4.78) and Eq. (4.79), and dividing by $k_{B} T$ we obtain the expression

$$
\frac{\tilde{D} \tilde{b}}{k_{B} T}=c_{1} c_{2} \Phi_{0} \times \frac{\bar{\gamma}_{\text {inter }}}{\gamma_{0}+\left(\bar{\gamma}_{\text {intra }}+\bar{\gamma}_{\text {inter }}\right)} .
$$

The product $\tilde{D} \tilde{b} / k_{B} T$ has the dimension of an inverse area and is independent of the time scale. Thus, its numerical value is directly comparable to that in the HRM and a successful mapping should yield $D b / k_{B} T=\tilde{D} \tilde{b} / k_{B} T$. Since we are interested in solving for the friction coefficients, we replace the LRM's actual value of this product by the known target value of the HRM, rearrange this equation as a linear combination of $\bar{\gamma}_{\text {inter }}$ and $\bar{\gamma}_{\text {intra }}$, and obtain

$$
\bar{\gamma}_{\text {inter }}\left(\frac{D b}{k_{B} T}-c_{1} c_{2} \Phi_{0}\right)+\bar{\gamma}_{\text {intra }}\left(\frac{D b}{k_{B} T}\right)+\gamma_{0}\left(\frac{D b}{k_{B} T}\right)=0 .
$$

\footnotetext{
${ }^{21}$ One could add an additional offset so that $\tilde{b}>0$ even if $\bar{\gamma}_{\text {inter }}=0$. But for brevity we omit such a term.
} 
Equation (4.81) reveals a problem. The friction coefficients $\bar{\gamma}_{\text {inter }}, \bar{\gamma}_{\text {intra }}$, and $\gamma_{0}$ are -for physical reasons- always positive, as well as the product $D b / k_{B} T$. Hence, a consistent, physically meaningful solution to this equation can only exist, if

$$
D b / k_{B} T<c_{1} c_{2} \Phi_{0}
$$

Thus, it all depends on the relative magnitude of the two constants $c_{1}$ and $c_{2}$. If they are too small, no physically meaningful solution for $\bar{\gamma}_{\text {inter }}$ and $\bar{\gamma}_{\text {intra }}$ exists, and $\tilde{D} \tilde{b} / k_{B} T$ cannot be matched to $D b / k_{B} T$ with the DPD thermostat alone.

Finally, we are looking for an expression relating the surface viscosity, $\tilde{\eta}$, to the thermostat's parameters. Fortunately, the shear viscosity of standard DPD fluids has been studied carefully and analytical expressions for two and three dimensions exist [138, 339]. Their derivations involve a sphere of action which is defined by higher moments of the DPD weighting functions. In our case, two such spheres exist and we define

$$
\left\langle R^{2}\right\rangle_{\text {intra }}=\frac{\left[r^{2} w_{\text {intra }}\right]}{\left[w_{\text {intra }}^{2}\right]} \text { and }\left\langle R^{2}\right\rangle_{\text {inter }}=\frac{\left[r^{2} w_{\text {inter }}\right]}{\left[w_{\text {inter }}^{2}\right]} .
$$

With these definitions, $\tilde{\eta}$ can be written in a convenient form as

$$
\tilde{\eta}=c_{3} m \Phi_{0} \times\left(\bar{\gamma}_{\text {intra }}\left\langle R^{2}\right\rangle_{\text {intra }}+\bar{\gamma}_{\text {inter }}\left\langle R^{2}\right\rangle_{\text {inter }}\right)
$$

where $c_{3}$ is another dimensionless coupling constant and $m$ is the mass of a molecule. To keep this equation linear in the effective friction coefficients, we have omitted a small kinetic term proportional to $\left(\bar{\gamma}_{\text {intra }}+\bar{\gamma}_{\text {inter }}\right)^{-1}$ [339].

A multiplication of Eq. (4.84) with Eq. (4.78) and a subsequent division by $k_{B} T$ lead to the dimensionless quantity

$$
\frac{\tilde{D} \tilde{\eta}}{k_{B} T}=c_{1} c_{3} m \Phi_{0} \times \frac{\bar{\gamma}_{\text {intra }}\left\langle R^{2}\right\rangle_{\text {intra }}+\bar{\gamma}_{\text {inter }}\left\langle R^{2}\right\rangle_{\text {inter }}}{\gamma_{0}+\left(\bar{\gamma}_{\text {intra }}+\bar{\gamma}_{\text {inter }}\right)} .
$$

Similarly to the previously treated case, a successful mapping should yield $\tilde{D} \tilde{\eta} / k_{B} T=D \eta / k_{B} T$. We therefore substitute $\tilde{D} \tilde{\eta} / k_{B} T$ by $D \eta / k_{B} T$, rewrite this expression as a linear combination of the effective friction coefficients, and arrive at

$$
\begin{aligned}
-\gamma_{0} \frac{D \eta}{k_{B} T} & =\bar{\gamma}_{\text {inter }}\left(\frac{D \eta}{k_{B} T}-c_{1} c_{3} m \Phi_{0}\left\langle R^{2}\right\rangle_{\text {inter }}\right) \\
& +\bar{\gamma}_{\text {intra }}\left(\frac{D \eta}{k_{B} T}-c_{1} c_{3} m \Phi_{0}\left\langle R^{2}\right\rangle_{\text {intra }}\right)
\end{aligned}
$$

For obtaining physically relevant results one of the two terms in brackets on the RHS must be negative. In contrast to Eq. (4.81) this condition is easier to 
fulfill because of the additional dependence on the action radii, $\left\langle R^{2}\right\rangle_{\text {inter }}$ and $\left\langle R^{2}\right\rangle_{\text {intra }}$. Their values can be set appropriately by choosing different weighting functions. Hence, by tweaking the DPD parameters, it is possible to set the LRM's surface viscosity to the desired values.

Matching $\tilde{D}, \tilde{b}$, and $\tilde{\eta}$ at once is only possible if $D b / k_{B} T$ is small enough. In this case, Eq. (4.81) and Eq. (4.86) form a set of two coupled linear equations with two unknowns $\left(\gamma_{\text {inter }}\right.$ and $\left.\gamma_{\text {intra }}\right)$. Although the equations still depend on the shape of the weighting functions, it is possible to find numerically exact solutions, if an ansatz for the weighting functions with a parameter is used.

\subsubsection{Mapping of the statics}

\section{The interaction potentials and their cutoff}

For matching the interaction potentials of the LRM, we set up dedicated simulations of the HRM for the two systems $\bar{\rho}^{*}=18$ and 40 in the $N P_{t} T$ ensemble with the standard DPD thermostat. Preequilibrated bilayer configurations with vanishing lateral tension in the fluid phase ${ }^{22}$ having $n=4680$ molecules and $N=16$ beads were used as initial configurations. Each simulation lasted $10^{4} \tau$ and the forces on the $n$ amphiphiles' center of mass, $\mathbf{f}_{I}\left(\mathbf{r}^{n N}\right), I=1, \ldots, n$ were saved every $2 \tau$.

The force-matching procedure outlined in Sec. 4.3.1 was used to calculate $U_{\text {intra }}(r)$ and $U_{\text {inter }}(r)$ from the recorded forces. We employed the block averaging approximation with 500 configurations per block, i.e., the final potentials are averages over ten independent estimates.

There are two degrees of freedom in this procedure. The first is the cutoff, $\Lambda$, for which we tried $\Lambda=2,3,4,5$, and $6 r_{c}$ and the second is the number of basis functions, $K$. Here we tried different values between $K=20$ and 100 , however, it turned out that $K$ is of minor importance. If the number of basis functions is too small for a certain $\Lambda$, then the potentials look jagged and acquire sharp edges; if it is too high, spurious oscillations pop up and the potentials look noisy. Between these extremes there is a broad range of possible values for $K$, and we chose $K=30$ for $\Lambda \leq 4 r_{c}$ and $K=50$ for $\Lambda \geq 5 r_{c}$. The right choice of $\Lambda$ is more involved and it is based on comparisons of the lateral pair correlation function, $g(r)$, the static density structure factor, $G(q)$, and the lateral pressure with those of the HRM. Another crucial criterion is the performance of the simulation which degrades with increasing $\Lambda$.

Figure 4.18 shows the two interaction potentials for $\bar{\rho}^{*}=18$ and $\Lambda=$ $3,4,5,6 r_{c}$ as well as the corresponding second order virial coefficients,

\footnotetext{
${ }^{22}$ The gel phase cannot be matched with this approach, because its configuration space is sampled only for lipid separations close to integer multiples of the lattice constant sufficiently.
} 


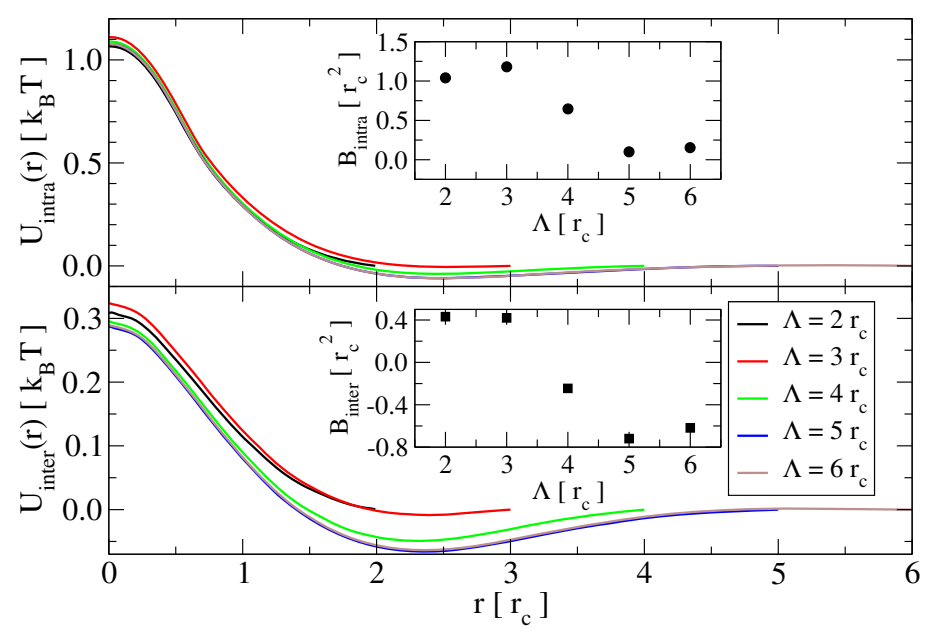

Figure 4.18: Interaction potentials $U_{\text {intra }}(r)$ (top) and $U_{\text {inter }}(r)$ (bottom) of the LRM as determined by the force-matching procedure for $\bar{\rho}^{*}=18, \Lambda=2,3,4,5,6 r_{c}$. The two insets show the second virial coefficients $B_{\text {intra }}(\Lambda)$ and $B_{\text {inter }}(\Lambda)$ calculated with Eq. (4.87), respectively.

$$
B_{x}=\pi \int_{0}^{\Lambda} r d r\left(1-e^{-\beta U_{x}(r)}\right)
$$

in the insets where $x \in\{$ intra, inter $\}$. At first glance $U_{\text {intra }}(r)$ and $U_{\text {inter }}(r)$ are soft, finite potentials which decay monotonously for $r<2 r_{c}$, enter a weak dip with a minimum at around $2.5 r_{c}$, and finally approach zero. The dip is the only source of attractive interactions between different molecules and therefore important for lowering the lateral pressure.

$U_{\text {intra }}(r)$ has a maximum at $r=0$ with an energy of $1.1 k_{B} T$ and a maximum depth of the dip of $0.06 k_{B} T$. If we use $\Lambda=2$ or $3 r_{c}$ the dip is absent. There is hardly any difference in $U_{\text {intra }}(r)$ between $\Lambda=5$ and $6 r_{c}$. The repulsive part of the other potential, $U_{\text {inter }}(r)$, is roughly a factor of 3 smaller than in $U_{\text {intra }}(r)$, but the dip has approximately the same depth with $0.07 k_{B} T$. From these findings we conclude that the essential features of the interactions are captured by $\Lambda=4$ and $5 r_{c}$. Larger values of $\Lambda$ lack any new features and slow down the whole simulation, thus, these values are disregarded in the remainder of this section.

Another interesting quantity for deciding on $\Lambda$ is the lateral pressure, $P_{t}=$ $L_{x}\left(P_{y y}+P_{z z}\right) / 2$. Ideally, one wants to reach the state where the membrane is tensionless, i.e., $P_{t}=0$. However, a look at the potentials in Fig. 4.18 shows that there are hardly any attractive interactions which reduce the lateral pressure. This can be quantified by inspecting the second order virial coefficients, 
Table 4.2: LRM's lateral pressure, $P_{t}$, in units of $k_{B} T / r_{c}^{2}$ for $\Lambda=6 r_{c}$. The attractive parts of $U_{\text {intra }}(r)$ and $U_{\text {inter }}(r)$ have been scaled by the factors $s_{\text {intra }}$ and $s_{\text {inter }}$ given in the first column and the first row, respectively. $\mathrm{X}$ marks a combination of factors where the system collapsed.

\begin{tabular}{|c|cccccccc|}
\hline & 1.00 & 1.01 & 1.02 & 1.05 & 1.10 & 1.15 & 1.20 & 1.25 \\
\hline 1.00 & 1.90 & 1.85 & 1.80 & & & & 0.70 & $\mathrm{X}$ \\
1.01 & 1.85 & 1.80 & & & 1.25 & 0.95 & & \\
1.02 & & 1.75 & 1.70 & & 1.20 & 0.90 & & \\
1.05 & & & & 1.30 & 1.05 & 0.70 & $\mathrm{X}$ & \\
1.10 & & 1.25 & 1.20 & 1.05 & 0.75 & $\mathrm{X}$ & $\mathrm{X}$ & \\
1.15 & & 1.00 & 0.90 & 0.75 & $\mathrm{X}$ & & & \\
1.20 & 0.75 & $\mathrm{X}$ & $\mathrm{X}$ & $\mathrm{X}$ & & & $\mathrm{X}$ & \\
1.25 & $\mathrm{X}$ & & & & & & & \\
\hline
\end{tabular}

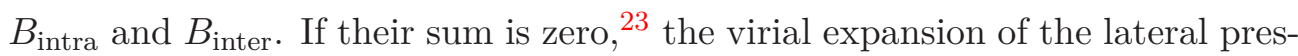
sure becomes $P_{t}=\Phi_{0} k_{B} T+\mathcal{O}\left(\Phi_{0}^{3}\right)$. Hence, $B_{\text {intra }}+B_{\text {inter }}$ must be negative to compensate the ideal gas term. This is only the case for $\Lambda>5 r_{c}$.

Obviously, the force-matching procedure in its current state misses some attractions that would lower $P_{t}$. Therefore, we increased the attractions manually. To this end $U_{\text {intra }}(r)$ and $U_{\text {inter }}(r)$ were split into a repulsive and an attractive part by a Weeks-Chandler-Andersen (WCA) decomposition [343]. The two attractive parts were scaled independently by a factor $s_{\text {intra }}$ or $s_{\text {inter }}$ in the range $1.00-1.60$, respectively, and the potentials were assembled again. Indeed, the succeeding simulations with $\Lambda=4,5,6 r_{c}$ revealed that $P_{t}$ was decreased (see Tab. 4.2).

Besides the fact that rescaling the attractive parts has a small influence on the static properties, e.g., $g(r)$ and $G(q)$ (to be discussed below), a new problem arose. Since the potential energies are finite, there is a critical line of rescaling factors at which the attractions win over the repulsions and the whole system becomes unstable, i.e., cluster-crystallization occurs. We stress that this is highly unphysical and a pure artifact of the finite interactions, but it sets a limit on $s_{\text {intra }}$ and $s_{\text {inter }}$. This critical line is located in the lower right half of Tab. 4.2 and separates the region with finite pressures from that where the system collapses. Noteworthily, even a small rescaling, i.e., $s_{\text {intra }}=1.05$ or $s_{\text {inter }}=1.05$, led for $\Lambda=5 r_{c}$ to a collapse. It seems that the force-matching procedure yields in this case interactions that are close to the critical line. The smallest values are $P_{t}=1.95 k_{B} T / r_{c}^{2}$ for $\Lambda=4 r_{c}\left(s_{\text {intra }}=s_{\text {inter }}=1.60\right)$, $P_{t}=1.15 k_{B} T / r_{c}^{2}$ for $\Lambda=5 r_{c}\left(s_{\text {intra }}=s_{\text {inter }}=1.00\right.$; all other were unstable $)$, and $P_{t}=0.70 k_{B} T / r_{c}^{2}$ for $\Lambda=6 r_{c}\left(s_{\text {intra }}=1.05\right.$ and $\left.s_{\text {inter }}=1.15\right)$.

\footnotetext{
${ }^{23}$ This corresponds to the Boyle temperature of a liquid.
} 
$U_{\text {intra }}(r)$, cf. Fig. 4.18, can be compared with the potential from Murtola et al., who map an atomistic model of a mixture of DPPC with cholesterol onto a fluid of discs by the inverse Monte Carlo method [332]. They employ a cutoff of $2.5 \mathrm{~nm}$ which translates to $3.2 r_{c}$ in our units, so that both potentials are directly comparable. Since their HRM features harsh repulsions, their forcematched potential is more repulsive than ours and exceeds $20 k_{B} T$ at $r=0 .{ }^{24}$ Similar to our result for $\Lambda=3 r_{c}$, their LRM potential decays monotonously lacking any visible, attractive dip. As a consequence it seems highly unlikely that a tensionless state can be obtained in their model.

One may also compare our potential to the work of Ayton and Voth who map an atomistic model of DMPC to a three-dimensional model where the lipids have an ellipsoidal shape [338]. For small distances the molecules interact via an anisotropic Gay-Berne potential. This potential has a harsh repulsion and diverges for $r \rightarrow 0$. For larger $r$ they interact via a spherical, force-matched potential. It has a cutoff of $2 \mathrm{~nm}\left(2.6 r_{c}\right)$ and possesses a broad attractive well with a depth of $\sim 2.5 k_{B} T$. Due to the anisotropic potential for small $r$ and the higher dimensionality, their potential is very different from ours and a direct comparison is, even though they use the same numerical method, impossible. Noteworthily, they are able to reach the tensionless state, $P_{t}=0$.

\section{Pair correlation function}

Another crucial check of the force-matching procedure is to compare the LRM's lateral pair correlation function with the HRM. With this check we can ensure that both models have the same molecular structure on small scales. Unlike Eq. (3.6) we define here two correlation functions,

$$
\begin{aligned}
& g_{\text {intra }}(r)=\frac{1}{\pi r n \Phi_{0}}\left\langle\sum_{i}^{n^{+}} \sum_{j \neq i}^{n^{+}} \delta\left(r-\left\|\mathbf{r}_{j}-\mathbf{r}_{i}\right\|\right)+\sum_{i} \sum_{j \neq i}^{n^{-}} \delta\left(r-\left\|\mathbf{r}_{j}-\mathbf{r}_{i}\right\|\right)\right\rangle \\
& g_{\text {inter }}(r)=\frac{2}{\pi r n \Phi_{0}}\left\langle\sum_{i}^{n^{+}} \sum_{j}^{n^{-}} \delta\left(r-\left\|\mathbf{r}_{j}-\mathbf{r}_{i}\right\|\right)\right\rangle,
\end{aligned}
$$

that measure the correlations within the same leaflet and between opposite leaflets. As before, $n^{ \pm}$denotes the number of lipids on the upper $(+)$or lower (-) leaflet, $n=n^{+}+n^{-}$, and $\Phi_{0}=n / A$. Both functions are normalized to 1 in the limit $r \rightarrow \infty$.

In Fig. 4.19 we compare both correlation functions for $\bar{\rho}^{*}=18$ and $\bar{\rho}^{*}=40$ and for $\Lambda=2,3,4,5 r_{c}$ to the ones obtained from the HRM. $g_{\text {intra }}(r)$ of the HRM starts at $r=0$ with $0.6\left(\bar{\rho}^{*}=18\right)$ and $0.8\left(\bar{\rho}^{*}=40\right)$, respectively,

\footnotetext{
${ }^{24}$ For the case with $0 \%$ cholesterol.
} 


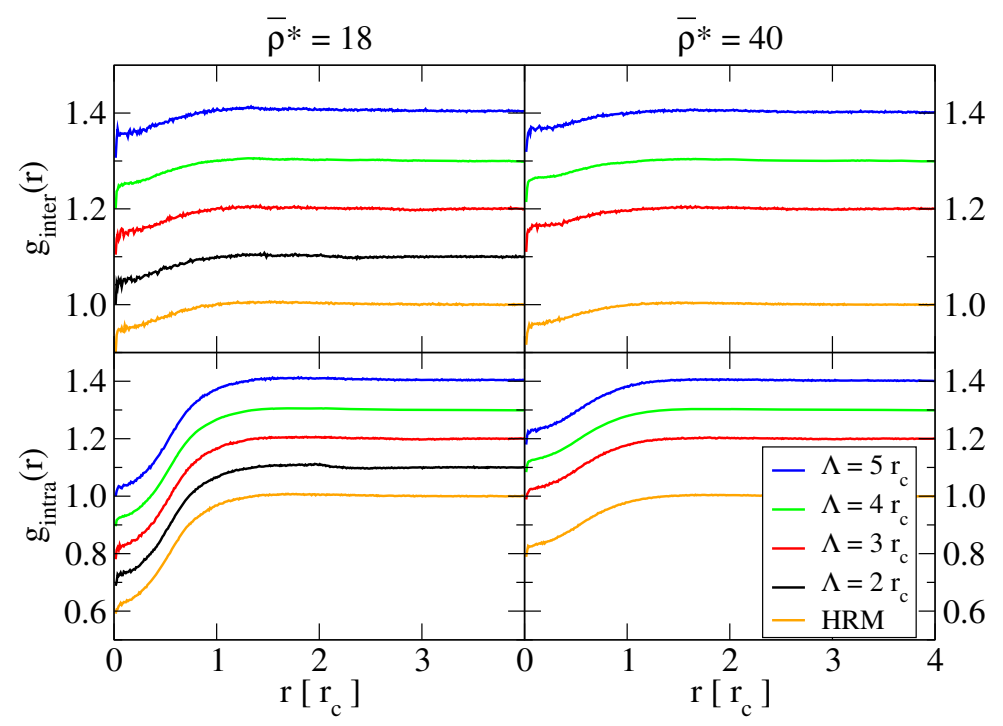

Figure 4.19: Lateral pair correlation functions $g_{\text {inter }}(r)$ (top) and $g_{\text {intra }}(r)$ (bottom) of the two systems $\bar{\rho}^{*}=18$ (left) and $\bar{\rho}^{*}=40$ (right) for different values of $\Lambda$ compared to the HRM. The curves for different $\Lambda$ have been stacked along the $y$-direction with a displacement of 0.1 .

indicating that there is a tremendous overlap of the molecules and only a very small excluded volume. There are hardly any packing oscillations except a little hump with 1.005 between $r=1.6$ and 2.0. The same is also true for $g_{\text {inter }}(r)$, but here the excluded volume is even smaller and at $r=0$ it starts at $0.94-0.95$. It is easy to imagine that these structureless functions can be matched readily. Indeed, the simulation of the LRM yields in all cases correlation functions that are very close to the HRM, i.e., one has to look carefully to see any differences. The humps of $g_{\text {intra }}(r)$ and $g_{\text {inter }}(r)$ are slightly overestimated at $\Lambda=2 r_{c}$. The force-matching procedure is capable of reproducing the right lateral pair correlation functions for $\Lambda>2 r_{c}$ and the structure on small scales is matched correctly. But since these functions are so structureless, $g_{\text {inter }}(r)$ and $g_{\text {intra }}(r)$ are no good indicators for the right choice of $\Lambda$.

\section{Static structure factor}

We now turn our attention to the large scale properties, that can be probed by the static structure factor,

$$
G(\mathbf{q})=\frac{1}{n}\left\langle\sum_{i=1}^{n} \sum_{j=1}^{n} e^{i \mathbf{q} \cdot\left(\mathbf{r}_{j}-\mathbf{r}_{i}\right)}\right\rangle .
$$




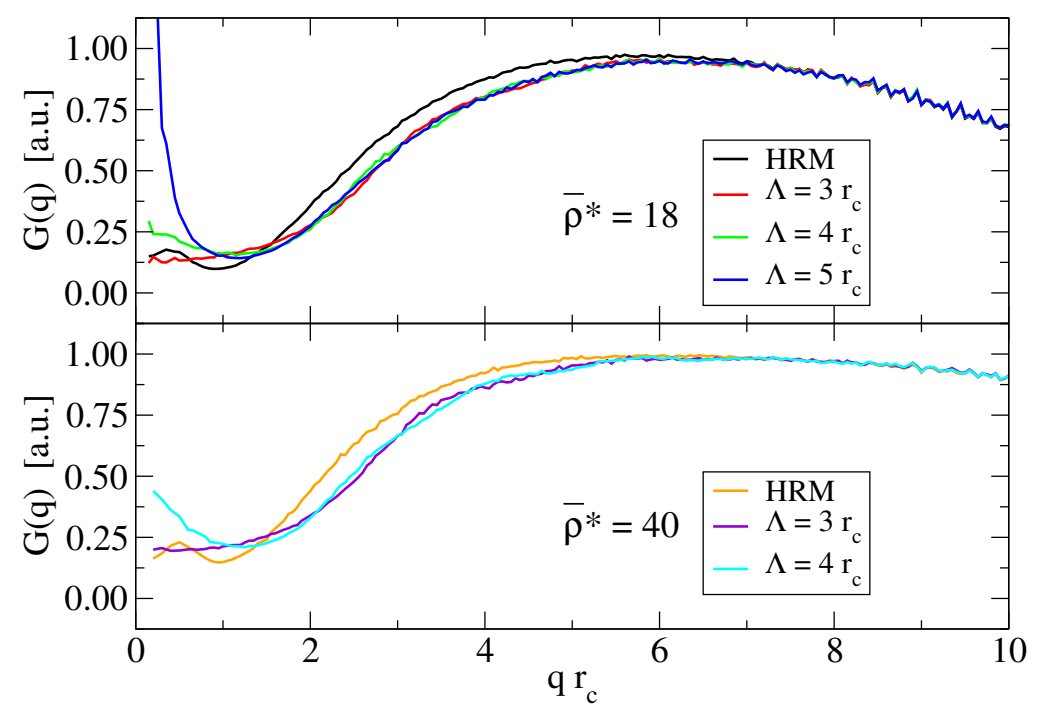

Figure 4.20: The radially averaged static structure factor, $G(q)$, of the two systems $\bar{\rho}^{*}=18$ (top) and $\bar{\rho}^{*}=40$ (bottom) for different values of $\Lambda$ compared to the HRM. The noise for $q r_{c}>8$ is caused by a radial averaging over a slightly anisotropic $G(q)$. This anisotropy is a numerical artifact caused by calculating $G(q)$ on a grid.

The spatial distribution of the amphiphiles lacks any long-range order and since there are hardly any packing effects, a wide distribution of molecular separations exists. Therefore, a plot of the HRM's $G(\mathbf{q})$ in the $q_{y}-q_{z}$-plane is rotationally symmetric and has a single broad ring without any isolated Bragg spots (see Fig. 4.20). In the limit $q \rightarrow 0, G(q)$ goes to a finite value. The results of the force-matching procedure show for $q r_{c}>2$ a similar distribution that is almost independent of $\Lambda$. Although the scattering intensity at $q r_{c}=6$ is the same as in the HRM, the curves are slightly shifted and have less intensity for small $q r_{c}$ which means that the LRM produces more disordered structures on larger scales.

Even though all $G(q)$ behave similarly for $q r_{c}>2$, there is a remarkable difference in the limit $q r_{c} \rightarrow 0$. It turns out that the limiting value, $G(q \rightarrow 0)$, which is proportional to the isothermal compressibility, increases with increasing $\Lambda$. This becomes particularly severe for $\Lambda=5 r_{c}$, where $G(q)$ diverges. Such a divergence indicates a liquid-vapor phase coexistence, as it is described by the Ornstein-Zernicke (OZ) theory [344], and certainly leads to an incorrect macroscopic behavior. This artifact of the force-matching procedure is highly unwanted and disqualifies the usage of $\Lambda \geq 5 r_{c}$. Even though $\Lambda=4 r_{c}$ exceeds the compressibility of the HRM, it is finite for both systems, $\bar{\rho}^{*}=18$ and $\bar{\rho}^{*}=40$, and at most twice as large. The smaller cutoffs $\Lambda=2 r_{c}$ (not shown) and $\Lambda=3 r_{c}$ yield good agreement with the HRM. 
In summary, the choice of $\Lambda$ is difficult because it has a strong impact on many aspects of the LRM. First, it is a crucial parameter for the performance of the simulations, because the number of interactions to be calculated scales with $\mathcal{O}\left(\Lambda^{2}\right)$ and large values degrade the performance significantly. Second, $\Lambda$ strongly influences the strength of the attractions and therefore the EOS. $\Lambda=2$ and $3 r_{c}$ lead to an almost purely repulsive potential, while $\Lambda=5$ and $6 r_{c}$ have a small attractive dip. However, large values of $\Lambda$ increase the isothermal compressibility of the LRM. For $\Lambda=5 r_{c}$ one already obtains a divergence of $G(q \rightarrow 0)$, which leads to the wrong macroscopic properties of the system. Since the pressure is too high for every set of parameters, and since the EOS was not included in the force-matching process, we expect the LRM to have an entirely different EOS from the HRM's. Consequently, such a system would never self-assemble into bilayers.

We conclude the discussion of the statics by stating that $\Lambda=4 r_{c}$ is -without any rescaling - the best compromise: it yields the right macroscopic properties, simulations are still efficient, the system does not collapse, and the pressure is as low as possible. Therefore, it will be used exclusively in the following discussion of the dynamics.

\subsubsection{Mapping of the dynamics}

The LRM's dynamics was studied for the system $\bar{\rho}^{*}=18$ and $\Lambda=4 r_{c}$, i.e., the quantities to be matched are $D b / k_{B} T \approx 1.3 \times 10^{-2} r_{c}^{-2}$ and $D \eta / k_{B} T \approx 0.48$ (cf. Tab. 4.1). Numerous DPD friction coefficients, $\gamma_{\text {intra }}$ and $\gamma_{\text {inter }}$, and two different (dissipative) weighting functions,

$$
\begin{aligned}
& w_{1}^{D}(r)= \begin{cases}(1-r / R)^{2}, & r<R \\
0, & r \geq R\end{cases} \\
& w_{2}^{D}(r)=\left\{\begin{array}{ll}
1, & r<R \\
0, & r \geq R
\end{array},\right.
\end{aligned}
$$

with variable cutoffs, $R \leq \Lambda$, were used in various combinations; the details are compiled in Tab. 4.3. Two different simulation runs were started for each set of DPD parameters: one equilibrium run for measuring $\tilde{D}$ from the bead's MSD, cf. Eq. (4.1), as well as $\tilde{b}$ via the Green-Kubo relation, cf. Eq. (4.36), and one non-equilibrium, RNEMD run for measuring $\tilde{\eta}$, cf. Eq. (4.30). Large values of $\bar{\gamma}_{\text {intra }}$ or $\bar{\gamma}_{\text {inter }}$ slowed down the relaxation significantly, thus we extended some runs for obtaining $\tilde{D}, \tilde{b}$, and $\tilde{\eta}$ with a comparable accuracy. The equilibrium runs took between 5000 and $24000 \tau$, and the RNEMD runs between 5000 and $56000 \tau$. 
Table 4.3: DPD friction coefficients and weighting functions for studying the LRM's dynamics. See Eqs. (4.90) and (4.91) for the definitions of the weighting functions.

\begin{tabular}{llccr}
\hline \hline$w_{\text {intra }} / R$ & $w_{\text {inter }} / R$ & $\gamma_{\text {intra }}$ & $\gamma_{\text {inter }}$ & \# runs \\
\hline$w_{1} / 4.0$ & $w_{1} / 4.0$ & $0.5-2.0$ & $4-16$ & 11 \\
$w_{1} / 3.5$ & $w_{1} / 3.5$ & $0.5-2.0$ & 4 & 2 \\
$w_{1} / 3.0$ & $w_{1} / 3.0$ & $0.5-6.0$ & $4-20$ & 10 \\
$w_{1} / 2.5$ & $w_{1} / 2.5$ & $0-4.0$ & $2-20$ & 15 \\
$w_{1} / 2.0$ & $w_{1} / 2.0$ & $0-4.0$ & $2-20$ & 24 \\
$w_{1} / 1.5$ & $w_{1} / 1.5$ & $0.5-2.0$ & 4 & 2 \\
$w_{1} / 1.0$ & $w_{1} / 1.0$ & $0.5-2.0$ & 4 & 2 \\
$w_{1} / 0.5$ & $w_{1} / 0.5$ & 2 & 4 & 1 \\
$w_{1} / 2.0$ & $w_{2} / 4.0$ & 1 & $12-20$ & 3 \\
$w_{1} / 2.5$ & $w_{2} / 4.0$ & 1 & $12-20$ & 3 \\
$w_{1} / 3.0$ & $w_{2} / 4.0$ & 1 & $12-20$ & 3 \\
$w_{2} / 4.0$ & $w_{2} / 4.0$ & 2 & $4-8$ & 3 \\
\hline \hline
\end{tabular}

\section{Diffusion}

First, we discuss the results of the measurement of $\tilde{D}$, which were obtained by the same method as in Sec. 4.1.2. In contrast to the HRM with three different regimes, the LRM's MSD function displays only two regimes: the ballistic and the freely diffusive one (see inset of Fig. 4.21). The intermediate, subdiffusive regime, which originates from the molecular connectivity, is missing.

The obtained diffusion coefficients are shown as a function of $\bar{\gamma}_{\text {intra }}+\bar{\gamma}_{\text {inter }}$ in the main panel of Fig. 4.21. We observe the expected universality, i.e., the data points collapse on a single curve that can be fitted by Eq. (4.78). This finding is in qualitative agreement with that from Lahmar and Rousseau [341] and demonstrates that $\bar{\gamma}_{\text {intra }}$ and $\bar{\gamma}_{\text {inter }}$ contribute equally to $\tilde{D}$. A least-squares fit with two parameters matches all but the largest values of $\bar{\gamma}_{\text {intra }}+\bar{\gamma}_{\text {inter }}$ and we obtain $c_{1}=2.46(2)$ and $\gamma_{0}=3.85 k_{B} T \tau / r_{c}^{2}$. The deviations from the asymptotic $\left(\bar{\gamma}_{\text {intra }}+\bar{\gamma}_{\text {inter }}\right)^{-1}$ power law for large friction are small.

\section{Intermonolayer friction}

We now turn to the discussion of the LRM's intermonolayer friction. Figure 4.22 depicts $\tilde{b}$ as a function of the effective friction between two leaflets, $\bar{\gamma}_{\text {inter }}$, for all sets of DPD parameters. The data points were obtained using the Green-Kubo relation with the same method as in Sec. 4.2.2. The measurement is straightforward and accurate estimates are available. All data points collapse on a single curve which is approximately linear and our linear expression, Eq. (4.79), is valid over three orders of magnitude. At the highest values, 


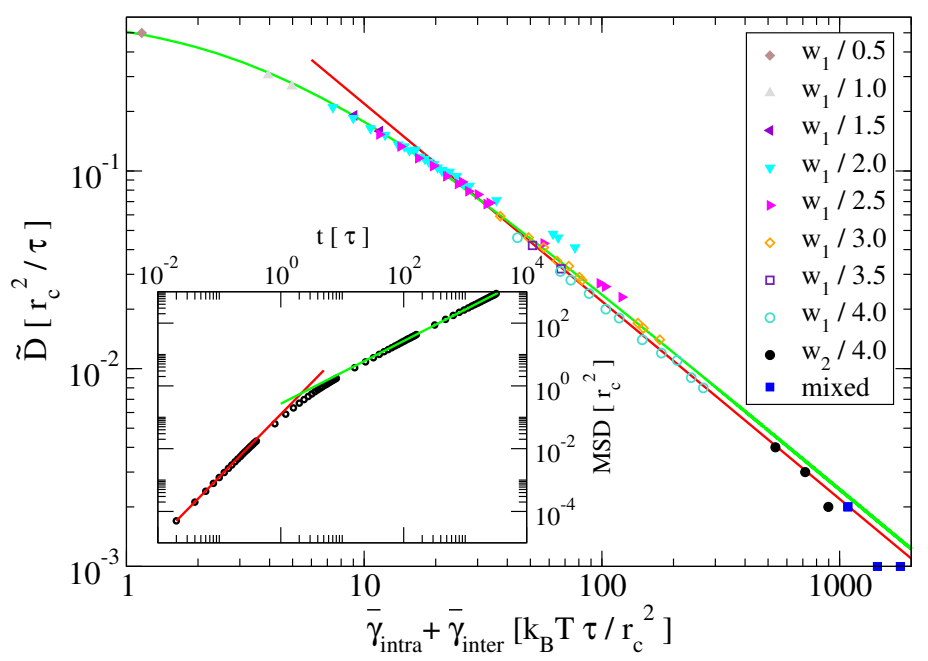

Figure 4.21: The LRM's diffusivity, $\tilde{D}$, as a function of the summed effective frictions, $\bar{\gamma}_{\text {intra }}+\bar{\gamma}_{\text {inter }}$. The data for different weighting functions and friction coefficients collapse on a single curve, described by Eq. (4.78) (green, solid line). The labels coincide with Tab. 4.3, "mixed" refers to the combinations where $w_{\text {intra }}=w_{1}$ and $w_{\text {inter }}=w_{2}$. The asymptotic power law is shown by the red, solid line. The inset shows the MSD for a typical simulation run $\left(w_{\text {intra }}(r)=w_{\text {inter }}(r)=w_{1}(r), R=2.5 r_{c}, \gamma_{\text {intra }}=4\right.$, $\left.\gamma_{\text {inter }}=2\right)$. The asymptote to the ballistic regime is shown in red, the one to the freely diffusive regime in green.

$\bar{\gamma}_{\text {inter }} \geq 10^{3}$, deviations from this linear behavior show up that may arise either from sampling difficulties or from non-linear effects. At very small values

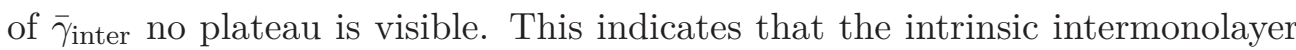
friction is negligible compared with the one induced by the thermostat. The influence of the intramonolayer DPD parameters, $\gamma_{\text {intra }}$ and $w_{\text {intra }}(r)$, on $\tilde{b}$ is virtually zero and even the data points for distinct intramonolayer weighting functions collapse on the same, universal curve.

A fit of the linear regime to Eq. (4.79) yields the dimensionless constant $c_{2}=1.02(2) \times 10^{-3}$. Hence,

$$
c_{1} c_{2} \Phi_{0}=8.93(2) \times 10^{-3} r_{c}^{-2}<D b / k_{B} T=13(1) \times 10^{-3} r_{c}^{-2}
$$

So even though it is possible to vary $\tilde{b}$ over more than three, and $\tilde{D}$ over more than two orders of magnitude in a controlled way, their variation is dependent and their product is always smaller than $D b / k_{B} T$. This means, the DPD thermostat slows the diffusion down faster than it is able to increase the intermonolayer friction. Hence, it is impossible to solve Eq. (4.81) for two positive friction coefficients. In other words, the HRM's intermonolayer friction is -on the relative time scale defined by the diffusivity- so high that it can- 


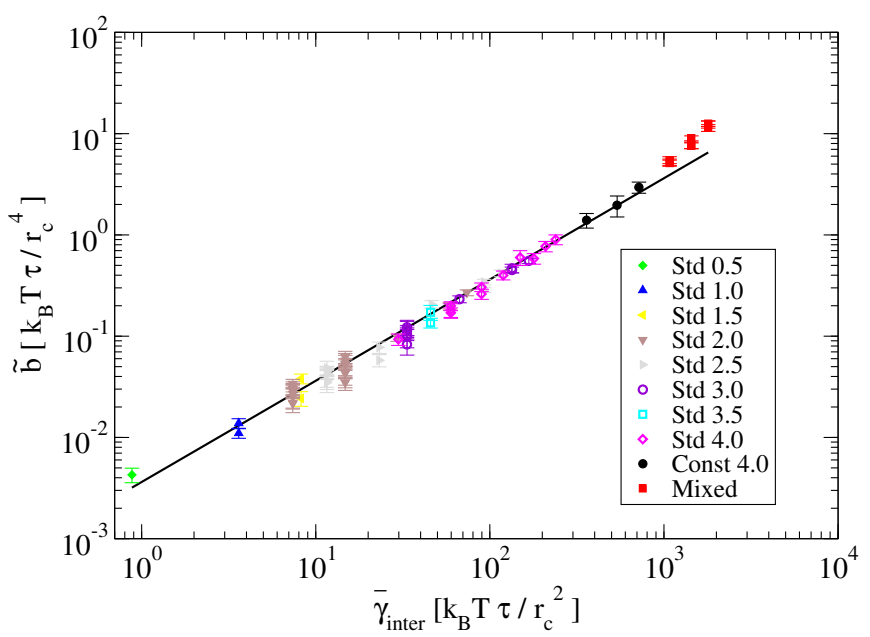

Figure 4.22: The LRM's intermonolayer friction coefficient, $\tilde{b}$, as a function of the effective intermonolayer DPD friction coefficient, $\bar{\gamma}_{\text {inter }}$. The names in the legend coincide with those in Fig. 4.21.

not be matched by adjusting the LRM's DPD parameters alone, although the difference is remarkably small.

It is difficult to judge if the LRM is capable of reproducing the intermonolayer friction of real existing lipid bilayers because of the two orders of magnitude difference between experiments and simulations (cf. Sec. 4.2.2). However, a direct comparison with the atomistic simulations from Wohlert and Edholm is feasible [278]. They found $b=2.8 \times 10^{6} \mathrm{~Pa} \mathrm{~s} / \mathrm{m}$ and $D=0.79 \times 10^{-11} \mathrm{~m}^{2} / \mathrm{s}$, which leads in our units to $D b / k_{B} T=5.48 \times 10^{-3} \mathrm{~nm}^{-2}=3.34 \times 10^{-3} r_{c}^{-2}$. This atomistic result is a factor 4 smaller than our coarse-grained result, and therefore smaller than $c_{1} c_{2} \Phi_{0}$. Hence, the LRM can be used to map this atomistic model. ${ }^{25}$

\section{Surface viscosity}

Finally, we move on to the discussion of the LRM's surface viscosity, $\tilde{\eta}$. We applied the RNEMD technique that allows efficient estimates for low to moderate friction. For very large friction, even this robust method becomes troublesome, because the velocity gradient gets very noisy and vanishes in short simulations.

Figure 4.23 shows $\tilde{\eta}$ as a function of $\left(\bar{\gamma}_{\text {intra }}\left\langle R^{2}\right\rangle_{\text {intra }}+\bar{\gamma}_{\text {inter }}\left\langle R^{2}\right\rangle_{\text {inter }}\right)$, cf. Eq. (4.84), for all DPD parameters. Although minor deviations exist, the data points seem to collapse onto a universal curve. This curve has a small negative slope for small values of the abscissa, but is essentially flat. A minimum at

\footnotetext{
${ }^{25}$ This requires, of course, different interaction potentials.
} 


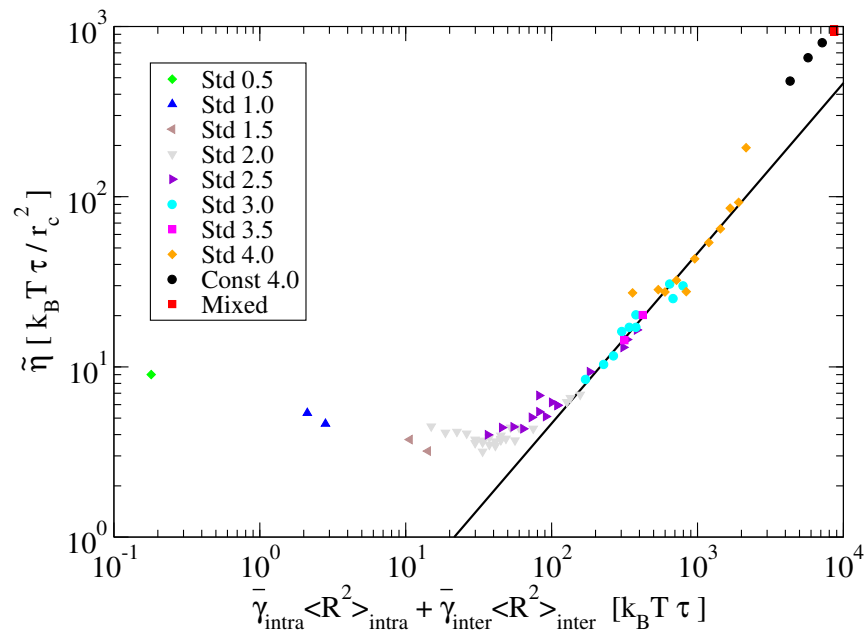

Figure 4.23: The surface viscosity, $\tilde{\eta}$, of the LRM as a function of $\bar{\gamma}_{\text {intra }}\left\langle R^{2}\right\rangle_{\text {intra }}+$ $\bar{\gamma}_{\text {inter }}\left\langle R^{2}\right\rangle_{\text {inter }}$. The names in the legend coincide with those in Fig. 4.21.

$\sim 3 k_{B} T \tau$ shows up, followed by a sharp rise by two orders of magnitude. The existence of a minimum and the decay at small values are, of course, not captured by our linear theory, since Eq. (4.84) neglects the kinetic contribution to the viscosity [339]. The sharp rise is approximately fitted with one parameter by the linear theory and we obtain a value for the coupling constant, $c_{3}=$ $8.136 \times 10^{-4}$. For very large friction, this linear theory becomes invalid, thus only the intermediate regime is captured acceptably. However, the quality of this fit is worse than that of the two other dynamical quantities. The existence of a universal curve for $\tilde{\eta}$ is, however, an important result and implies that one can predict $\tilde{\eta}$ 's value approximately from the knowledge of the thermostat's parameters.

We conclude the discussion of the dynamics by a brief look at the accuracy of this mapping procedure. For the sake of simplicity we take the results of one of the already existing simulation runs: $w_{\text {intra }}(r)=w_{\text {inter }}(r)=w_{1}(r)$, $R=2.5 r_{c}, \gamma_{\text {intra }}=4$, and $\gamma_{\text {inter }}=2$. This parameterization should be valid for mapping the HRM's surface viscosity to the LRM. Using $c_{1}$ and $\gamma_{0}$ from above, we compute the expected diffusion coefficient, $\tilde{D}_{\exp }=0.0672 r_{c}^{2} / \tau$. The value obtained from the simulation, $\tilde{D}_{\text {sim }}=0.0677 r_{c}^{2} / \tau$, is in excellent agreement with this prediction. The expected surface viscosity, $\tilde{\eta}_{\exp }=7.03 k_{B} T \tau / r_{c}^{-2}$ is a bit higher than the measured value, $\tilde{\eta}_{\text {sim }}=5.9 k_{B} T \tau / r_{c}^{-2}$. We obtain for the expected dimensionless mapping parameter, $\left(\tilde{D} \tilde{\eta} / k_{B} T\right)_{\exp }=0.47$, and for the measured one, $\left(\tilde{D} \tilde{\eta} / k_{B} T\right)_{\text {sim }}=0.40(5)$. With this simple mapping scheme, one can map the product $D \eta / k_{B} T$ from the HRM to the LRM with an accuracy of two significant digits. 


\subsubsection{Conclusions}

In this section we established a two-dimensional representation of our soft, solvent-free coarse-grained model for amphiphilic bilayers. The molecules are represented by soft, structureless discs that interact via pair potentials. We employed the force-matching procedure to find interaction potentials which closely mimic the effective interactions between lipids in the original model. The most important parameter in this matching is the cutoff of the interactions, $\Lambda$. We investigated the effect of different values of $\Lambda$ on the lateral pair correlation functions, the static structure factor, the second order virial coefficients, and the lateral pressure of our model. The short-ranged liquid structure is obtained easily, but the disc model seems to be unable to reproduce longer-ranged correlations correctly and large-scale properties crucially depend on $\Lambda$. The lateral pressure was in all cases too high, which can be attributed to the lack of any significant attractive tails in the interaction potentials. An amplification of the attraction by means of a WCA-decomposition lowered the pressure, but introduced instabilities. The best compromise for all these quantities has been found for $\Lambda=4.0 r_{c}$.

The DPD thermostat was used to recover the amount of friction which was lost by removing the intramolecular degrees of freedom. We used different weighting functions and different friction coefficients for the interactions within the same leaflet and within opposite leaflets. The choice of these degrees of freedom of the thermostat affected the dynamical quantities differently. The diffusivity, $\tilde{D}$, only depends on the sum of the effective intra- and interleaflet contributions; the weighting functions enter as a numerical prefactor. Hence, every value of $\tilde{D}$ can be obtained by manipulating only the friction coefficients. The surface viscosity, $\tilde{\eta}$, depends non-linearly on the sum of the intra- and interleaflet frictions, each scaled by a factor that includes various moments of the weighting functions. This dependency offers the possibility of controlling $\tilde{\eta}$ 's value through the shape of the two weighting functions. Finally, the intermonolayer friction, $\tilde{b}$, depends only on the effective interleaflet friction. This dependency is linear in the friction over several orders of magnitude. The DPD thermostat, with these four degrees of freedom, is a versatile tool as long as only one of these three quantities is required to have a predefined value. The universal behavior of $\tilde{D}, \tilde{b}$, and $\tilde{\eta}$ on the parameters facilitates a straightforward mapping and saves a significant amount of computing time.

For a physically meaningful mapping, at least two dynamical quantities must be considered at the same time. Here, one of them is $\tilde{D}$ which serves as a conversion factor for the unit of time. We identified $D b / k_{B} T$ and $D \eta / k_{B} T$ as reasonable mapping parameters, because they do not depend on the arbitrary timescale and because the units of length and energy agree in both models. We demonstrated that an upper boundary for $D b / k_{B} T$ exists, and that values 
exceeding this boundary cannot be obtained from our thermostat. The product with the surface viscosity, $D \eta / k_{B} T$, is easier to obtain, since it involves higher moments of the weighting functions which serve as additional tuning parameters.

The mapping of the HRM's dynamics onto the LRM was only partially successful. Though it was possible to obtain $\tilde{D} \tilde{\eta} / k_{B} T \approx D \eta / k_{B} T, D b / k_{B} T$ was slightly too high to be mapped. However, an atomistic simulation from a different group yielded a value of $D b / k_{B} T$ below the threshold, thus the LRM could in principle be used to map such a model. Since the intermonolayer friction and the diffusivity increase with an increasing molecular density, $\bar{\rho}^{*}$, the LRM is unable to describe the dissipation mechanisms of polymeric membranes. It seems that it is only capable of describing the intermonolayer friction of weakly interdigitated objects, e.g., lipids or soft rods. This can be also conceived from another point of view: the LRM crucially depends on the assumption of two weakly coupled leaflets. This assumption is justified in lipid bilayers, however, with an increasing molecular density the degree of interdigitation between the two monolayers, and thus the coupling, also increases and the basic assumption of the LRM fails.

We conclude from these findings that strongly coarse-grained models lack the ability to generate large values of the intermonolayer friction. The HRM, in turn, is able to generate much greater values of it, but lacks the ability of generating small ones. Hence both models are complementary in this respect. It would be interesting to see, if the molecular discretization, $N$, provides another means for modifying $b$ in the HRM. In this case one would end up with a parameter, that is more flexible than the DPD thermostat and that can be used to tune $b$ to high and low values easily. 


\section{Conclusions and outlook}

"Further, science is a collaborative effort."

(John Bardeen)

Collective phenomena in lipid bilayer membranes, like self-assembly, pore formation and fusion, lateral phase separation, collective diffusion, or phase transitions, play an important role in many biological processes. These phenomena involve hundreds to thousands of molecules and the characteristic time and length scales are microseconds and micrometers. Polymer membranes of diblock copolymers can be tailored to meet a wide range of applications and are much more robust than their lipid counterparts. However, they possess the same relevant interactions at the mesoscale, i.e., the scale between the monomeric repeat unit and the extension of the entire molecule, and thus show similar collective phenomena. Due to the higher molecular weight, the characteristic time and length scales are one to two orders of magnitude larger.

These scales, microseconds and micrometers, are often difficult to observe in experiments, so that computer models are used to aid in the interpretation of experimental results or to predict the outcome of certain measurements. Atomistic models, where all molecules are described in great chemical detail, are hardly suited to the study of these mesoscopic collective phenomena, therefore one often resorts to a coarse-grained description.

In Chapter 2, we have developed a coarse-grained, solvent-free model which smoothly interpolates between lipid and polymer membranes. Our soft, nonbonded interactions are based on a classical, weighted density functional which is a third-order expansion in weighted molecular densities. The thermodynamic properties and the local structure of our model decouple within the mean-field approximation, and can be controlled independently by the expansion coefficients and two weighting functions. By calculating the expansion coefficients from simple physical principles, which involved only quantities that are accessible from experiments, we impart a transparent interpretation onto these coefficients. Appropriate weighting functions are selected to create weak packing effects. The bonded, intramolecular interactions include simple harmonic potentials to mimic the connectivity and bond-angle potentials to restrict conformational fluctuations. We employ multibody dissipative particle dynamics to integrate the beads' equations of motion. 
A parallel simulation code, based on the force-decomposition scheme, was written allowing for the simulation of our model within the canonical or the tensionless ensemble. The program scales well up to $64 \mathrm{CPUs}$, so that large systems of $10^{4}-10^{5}$ molecules can be simulated. Linear dimensions of $100 \mathrm{~nm}$ and time intervals of $10 \mu$ s are accessible, thus, our model allows for a qualitative study of collective phenomena.

Chapter 3 of this work discusses the static properties of our model. Here, we confirm that it accurately represents polymer as well as lipid bilayers. Depending on the interaction coefficients, our model shows self-assembly into spherical, cylindrical, or wormlike micelles, as well as to bilayers or inverted structures. The lipid bilayer phase diagram comprises the fluid phase $\left(L_{\alpha}\right)$ as well as several gel phases $\left(L_{\beta}, L_{\beta^{\prime}}, L_{\beta I}\right)$. We did not observe a ripple phase $\left(P_{\beta^{\prime}}\right)$. The bending rigidity was found to be around $20 k_{B} T$, which is high enough for biomembranes (without proteins) to be simulated.

In a subsequent discussion we turned to the discontinuous main phase transition between the fluid and a gel phase $\left(L_{\beta}\right)$. We presented a method based on a non-Boltzmann sampling scheme to locate precisely the point of this phase transition. In comparison to the trivial way of finding the phase transition point by looking at the center and the width of the corresponding hysteresis loop, this method gives an estimate that is twenty times more accurate. In addition we studied the lateral demixing of fluid and gel domains and measured the line tension between such domains. It is very close to experimental values, i.e., $\sim 10 \mathrm{pN}$. It was demonstrated that two different definitions, the thermodynamic and the bare line tension, exist which differ by a fluctuation term that depends on the length scale.

The coarse-grained dynamics was studied in Chapter 4. We carefully analyzed the diffusion of the lipids by measuring the dynamic structure factor, the velocity autocorrelation function, and the mean-square displacement of the beads and the centers of mass. This study revealed that there is hardly any concerted motion of the centers of mass. In particular, no flow pattern like in atomistic models is observable [92], the lipids move in unison only for very short time intervals and their motion decorrelates quickly. The dynamic structure factors offered insights into the spectrum of collective excitations and the dispersion relations of the bilayers; a value for the speed of sound was extracted. Noteworthily, the flipflop rate, i.e., the rate of transversal diffusion of the lipids across the bilayer, is vanishingly small.

Another large part of the study was dedicated to the dissipation mechanisms. In contrast to atomistic simulations with an explicit, viscous solvent, there are only two instead of three mesoscopic dissipation mechanisms in our solvent-free model: the intermonolayer friction and the surface viscosity. Both quantities have not yet been measured in a solvent-free model, and two methods for calculating each one were presented. In particular we derived a new 
Green-Kubo relation for measuring the intermonolayer friction and presented a modified version of the Seifert-Langer theory $[84,85]$ that describes the time evolution of undulation modes. This modification included an inertial term which was neglected in the original theory. This term led to propagating undulation modes and explained the observed time evolution of the undulations in our solvent-free model very well. The obtained numerical value of the surface viscosity, $\eta \approx 3.9 \times 10^{-10} \mathrm{~Pa} \mathrm{~m} \mathrm{~s}$, matches the one from experiments [287-289]. The intermonolayer friction's value, $b \approx 1.6 \times 10^{6} \mathrm{~Pa} \mathrm{~s} / \mathrm{m}$, agrees with other simulations [278, 304], but differs from experiments by two orders of magnitude [298-300]. The reason for this discrepancy has not been resolved yet.

The impact of coarse-graining on the static properties is well understood, but its influence on the dynamics is still under active research. At present no practical scheme for mapping the dynamics of an atomistic model onto a coarse-grained model exists. Nevertheless, it is interesting to see how the coarse-graining procedure affects the dynamics. In a final part of Chapter 4 we devised another, even more coarse-grained model for lipid bilayers where entire molecules are represented as two-dimensional soft particles that move only laterally. We studied the question to what extent the DPD thermostat can be used to yield the same dissipation constants, measured on a relative time scale set by the lateral diffusion, as in the full bead-spring model. It turns out that such a mapping is indeed possible, as long as these constants are not too high. The surface viscosity can be mapped easily, whereas mapping the intermonolayer friction is far more challenging.

In this work we have exclusively studied single-component bilayers that served as a good testing bed for our new model. However, biomembranes as well as synthesized lipid bilayers always contain more than one species, e.g., DPPC with cholesterol, so that the next step in line of this work is certainly an extension to more than one species. This would open up the possibility of studying phase diagrams of lipid mixtures, line tensions between domains of different compositions, or the elastic properties as functions of the lipid composition. One could also investigate if the collective diffusion changes, or how the intermonolayer friction and the surface viscosity depend on the composition. Such an extension is -from a technical point of view-straightforward: an addition of a single bead species to the excess free energy functional, Eq. (2.3), suffices.

The equilibrium properties of membranes are well-understood. In their natural surroundings, biomembranes are permanently subjected to external forces, e.g., osmotic pressure differences, significant electric fields, or membrane proteins, which keep them out of equilibrium. Such forces induce stress or curvature in the membranes and may lead to large deformations. Thus, another continuation of this work is to study such deformations. One could ask questions concerning the curvature's influence on the motion of the molecules, or its effect on the phase diagram. 
At the moment, the model is already used in two other projects. The first investigates the adhesion, rupture, and spreading of vesicles on solid substrates, and the related pathways. In the second project a simplified model for a protein has been devised and the protein's influence on the pathway of membrane fusion is analyzed.

Summarizing the salient points of this thesis, we have presented a general, very efficient solvent-free, coarse-grained model for the simulation of lipid and polymer bilayers that describes the statics and dynamics realistically. The model has proven useful in this work, and hopefully it will do the same in future work. "Science is a collaborative effort", and with this thesis we hope to have created a good starting point for further research on collective phenomena in lipid and polymer membranes. 


\section{A Interaction coefficients}

Table A.1: Frequently occurring interaction parameters. Not shown are $v_{B B}=0.1$, $w_{B B B}=0, w_{A A A}=w_{A A B}=w_{A B B}$.

\begin{tabular}{ccc|ccc}
\hline \hline $\bar{\rho}^{*}$ & $\kappa N$ & $\chi N$ & $v_{A A}$ & $v_{A B}$ & $w_{A A A}$ \\
\hline 17 & 100 & 30 & -12.1176 & -4.24412 & 0.529412 \\
18 & 100 & 30 & -11.4444 & -4.00556 & 0.472222 \\
20 & 100 & 30 & -10.3 & -3.6 & 0.3825 \\
40 & 100 & 30 & -5.15 & -1.775 & 0.095625 \\
\hline \hline
\end{tabular}

Table A.2: Calculated intramolecular parameters for $N=10, \ell_{0}=0$. Recommended parameters are in bold.

\begin{tabular}{lcr}
\hline \hline$R_{\mathrm{eo}}\left[r_{c}\right]$ & $k_{b}\left[k_{B} T\right]$ & $k_{s}\left[k_{B} T / r_{c}^{2}\right]$ \\
\hline 2.0 & 2 & 15.001 \\
$\mathbf{2 . 0}$ & $\mathbf{3}$ & $\mathbf{2 0 . 5 3 7}$ \\
2.0 & 4 & 25.288 \\
2.0 & 5 & 28.635 \\
2.2 & 5 & 23.969 \\
2.5 & 2 & 9.662 \\
$\mathbf{2 . 5}$ & $\mathbf{3}$ & $\mathbf{1 3 . 0 3 3}$ \\
2.5 & 4 & 16.195 \\
2.5 & 5 & 18.590 \\
2.8 & 5 & 14.650 \\
3.0 & 5 & 12.745 \\
3.5 & 5 & 9.405 \\
\hline \hline
\end{tabular}


Table A.3: Calculated intramolecular parameters for $N=16$. Used parameters are in bold.

\begin{tabular}{|c|c|c|c|}
\hline$R_{\mathrm{eo}}\left[r_{c}\right]$ & $k_{b}\left[k_{B} T\right]$ & $\ell_{0}\left[r_{c}\right]$ & $k_{s}\left[k_{B} T / r_{c}^{2}\right]$ \\
\hline 3.5 & 0 & 0 & 3.673 \\
\hline 3.5 & 4 & 0 & 16.026 \\
\hline 3.5 & 5 & $\mathbf{0}$ & 19.089 \\
\hline 3.5 & 5 & 0.1 & 25.516 \\
\hline 3.5 & 5 & 0.2 & 38.793 \\
\hline 3.5 & 5 & 0.3 & $\sim 90$ \\
\hline 3.5 & 6 & 0 & 21.658 \\
\hline 3.5 & 6 & 0.1 & 29.798 \\
\hline 3.5 & 6 & 0.2 & 48.128 \\
\hline 3.5 & 6 & 0.3 & $\sim 135$ \\
\hline 3.5 & 7 & 0 & 24.024 \\
\hline 3.5 & 7 & 0.1 & 33.382 \\
\hline 3.5 & 7 & 0.2 & 56.438 \\
\hline 3.5 & 7 & 0.3 & $\sim 230$ \\
\hline 3.5 & 8 & 0 & 26.033 \\
\hline 3.5 & 8 & 0.1 & 36.778 \\
\hline 3.5 & 8 & 0.2 & 64.539 \\
\hline 3.5 & 8 & 0.3 & $\sim 375$ \\
\hline 3.5 & 9 & 0 & 27.611 \\
\hline 3.5 & 9 & 0.1 & 39.598 \\
\hline 3.5 & 9 & 0.2 & 72.596 \\
\hline 3.5 & 9 & 0.3 & $\sim 1000$ \\
\hline 3.5 & 10 & 0 & 29.007 \\
\hline 3.5 & 10 & 0.1 & 42.136 \\
\hline 3.5 & 10 & 0.2 & 80.247 \\
\hline 5.0 & 20 & 0 & 18 \\
\hline
\end{tabular}




\section{Bibliography}

[1] H. Lodish, A. Berk, C. A. Kaiser, M. Krieger, M. P. Scott, and A. Bretscher. Molecular Cell Biology. Palgrave Macmillan, New York, 2007.

[2] O. G. Mouritsen. Life - As a Matter of Fat. Springer, Berlin, 2005.

[3] J. N. Israelachvili. Intermolecular and Surface Forces. Academic Press, London, second edition, 1991.

[4] B. M. Discher, Y.-Y. Won, D. S. Ege, J. C.-M. Lee, F. S. Bates, D. E. Discher, and D. A. Hammer. Polymersomes: Tough vesicles made from diblock copolymers. Science, 284:1143-1146, 1999. doi: $10.1126 /$ science.284.5417.1143.

[5] I. W. Hamley. Nanoshells and nanotubes from block copolymers. Soft Matter, 1:36-43, 2005. doi: 10.1039/B418226J.

[6] M. Massignani, H. Lomas, and G. Battaglia. Polymersomes: A synthetic biological approach to encapsulation and delivery. Adv. Polym. Sci, 229: 115-154, 2010. doi: 10.1007/12_2009_40.

[7] M. Malinova, S. Belegrinou, D. de Bruyn Oubouter, and W. Meier. Biomimetic block copolymer membranes. Adv. Polym. Sci, 224:113-165, 2010. doi: 10.1007/12_2008_10.

[8] L. V. Chernomordik and M. M. Kozlov. Mechanics of membrane fusion. Nat. Struct. Mol. Biol., 15:675-683, 2008. doi: 10.1038/nsmb.1455.

[9] J. Zimmerberg and L. V. Chernomordik. Membrane fusion. Adv. Drug Deliv. Rev., 38:197-205, 1999. doi: 10.1016/S0169-409X(99)00029-0.

[10] B. R. Lentz, V. Malinin, M. E. Haque, and K. Evans. Protein machines and lipid assemblies: current views of cell membrane fusion. Curr. Opin. Struct. Biol., 10:607-615, 2000. doi: 10.1016/S0959-440X(00)00138-X.

[11] R. Jahn and H. Grubmüller. Membrane fusion. Curr. Opin. Cell Biol., 14:488-495, 2002. doi: 10.1016/S0955-0674(02)00356-3. 
[12] M. Müller, K. Katsov, and M. Schick. A new mechanism of model membrane fusion determined from Monte Carlo simulation. Biophys. J., 85: 1611-1623, 2003. doi: 10.1016/S0006-3495(03)74592-5.

[13] L. Yang and H. W. Huang. Observation of a membrane fusion intermediate structure. Science, 297:1877-1879, 2002. doi: $10.1126 /$ science. 1074354 .

[14] L. Luo and A. Eisenberg. Thermodynamic size control of block copolymer vesicles in solution. Langmuir, 17:6804-6811, 2001. doi: 10.1021/la0104370.

[15] A. A. Choucair, A. H. Kycia, and A. Eisenberg. Kinetics of fusion of polystyrene-b-poly(acrylic acid) vesicles in solution. Langmuir, 19:10011008, 2003. doi: 10.1021/la026187k.

[16] Y. Mai, Y. Zhou, and D. Yan. Real-time hierarchical self-assembly of large compound vesicles from an amphiphilic hyperbranched multiarm copolymer. Small, 3:1170-1173, 2007. doi: 10.1002/smll.200600733.

[17] T. Baumgart, S. T. Hess, and W. W. Webb. Imaging coexisting fluid domains in biomembrane models coupling curvature and line tension. Nature, 425:821-824, 2003. doi: 10.1038/nature02013.

[18] S. Mukherjee and F. R. Maxfield. Membrane domains. Ann. Rev. Cell Devel. Biol., 20:839-866, 2004. doi: 10.1146/annurev.cellbio.20.010403.095451.

[19] S. L. Veatch and S. L. Keller. Seeing spots: Complex phase behavior in simple membranes. Biochim. Biophys. Acta - Mol. Cell Res., 1746: 172-185, 2005. doi: 10.1016/j.bbamcr.2005.06.010.

[20] R. Koynova and M. Caffrey. Phases and phase transitions of the phosphatidylcholines. Biochim. Biophys. Acta Rev. Biomembranes, 1376:91145, 1998. doi: 10.1016/S0304-4157(98)00006-9.

[21] J. G. E. M. Fraaije, C. A. van Sluis, A. Kros, A. V. Zvelindovsky, and G. J. A. Sevink. Design of chimaeric polymersomes. Faraday Discussion, 128:355-361, 2005. doi: 10.1039/b403187c.

[22] D. A. Christian, A. W. Tian, W. G. Ellenbroek, I. Levental, K. Rajagopal, P. A. Janmey, A. J. Liu, T. Baumgart, and D. E. Discher. Spotted vesicles, striped micelles and janus assemblies induced by ligand binding. Nat. Mater., 8:843-849, 2009. doi: 10.1038/NMAT2512. 
[23] C. LoPresti, M. Massignani, C. Fernyhough, A. Blanazs, A. J. Ryan, J. Madsen, N. J. Warren, S. P. Armes, A. L. Lewis, C. S. Chirasatitsin, A. J. Engler, and G. Battaglia. Controlling polymersome surface topology at the nanoscale by membrane confined polymer/polymer phase separation. ACS Nano, 5:1775-1784, 2011. doi: 10.1021/nn102455z.

[24] J. Nam, P. A. Beales, and T. K. Vanderlick. Giant phospholipid/block copolymer hybrid vesicles: Mixing behavior and domain formation. Langmuir, 27:1-6, 2011. doi: 10.1021/la103428g.

[25] S. Chien. Red cell deformability and its relevance to blood flow. Ann. Rev. Physiol., 49:177-192, 1987. doi: 10.1146/annurev.ph.49.030187.001141.

[26] J. Deschamps, V. Kantsler, E. Segre, and V. Steinberg. Dynamics of a vesicle in general flow. Proc. Natl. Acad. Sci. USA, 106:11444-11447, 2009. doi: 10.1073/pnas.0902657106.

[27] M. Abkarian and A. Viallat. Dynamics of vesicles in a wall-bounded shear flow. Biophys. J., 89:1055-1066, 2005. doi: 10.1529/biophysj.104.056036.

[28] S. R. Keller and R. Skalak. Motion of a tank-treading ellipsoidal particle in a shear flow. J. Fluid. Mech., 120:27-47, 1982. doi: $10.1017 /$ S0022112082002651.

[29] R. Tran-Son-Tay, S. P. Sutera, and P. R. Rao. Determination of red blood cell membrane viscosity from rheoscopic observations of tank-treading motion. Biophys. J., 46:65-72, 1984. doi: 10.1016/S0006-3495(84)83999-5.

[30] H. Noguchi and G. Gompper. Dynamics of fluid vesicles in shear flow: Effect of membrane viscosity and thermal fluctuations. Phys. Rev. E, 72: 011901, 2005. doi: 10.1103/PhysRevE.72.011901.

[31] E. Evans, A. Yeung, R. Waugh, and J. Song. Dynamic coupling and nonlocal curvature elasticity in bilayer membranes. In R. Lipowsky, D. Richter, and K. Kremer, editors, The structure and conformation of amphiphilic membranes, pages 148-153, Berlin, 1992. Springer.

[32] E. Evans and A. Yeung. Hidden dynamics in rapid changes of bilayer shape. Chem. Phys. Lipids, 73:39-56, 1994. doi: 10.1016/0009-3084(94)90173-2.

[33] G. P. Robbins, R. L. Saunders, J. B. Haun, J. Rawson, M. J. Therien, and D. A. Hammer. Tunable leuko-polymersomes that adhere specifically to inflammatory markers. Langmuir, 26:14089-14096, 2010. doi: 10.1021/la1017032. 
[34] G. P. Robbins, D. Lee, J. S. Katz, P. R. Frail, M. J. Therien, J. C. Crocker, and D. A. Hammer. Effects of membrane rheology on leukopolymersome adhesion to inflammatory ligands. Soft Matter, 7:769-779, 2011. doi: 10.1039/c0sm00554a.

[35] J.-P. Ryckaert, G. Ciccotti, and H. J. C. Berendsen. Numerical integration of cartesian equations of motion of a system with constraints: molecular dynamics of $n$-alkanes. J. Comp. Phys., 23:327-341, 1977. doi: 10.1016/0021-9991(77)90098-5.

[36] H. C. Andersen. Rattle: A "velocity" version of the shake algorithm for molecular dynamics calculations. J. Comput. Phys., 52:24-34, 1983. doi: 10.1016/0021-9991(83)90014-1.

[37] B. Hess, H. Bekker, H. J. C. Berendsen, and J. G. E. M. Fraaije. LINCS: A linear constraint solver for molecular simulations. J. Comput. Chem., 18:1463-1472, 1997. doi: 10.1002/(SICI)1096-987X(199709)18:12<1463::AID-JCC4>3.0.CO;2-H.

[38] U. Essmann, L. Perera, M. L. Berkowitz, T. Darden, H. Lee, and L. G. Pedersen. A smooth particle mesh Ewald method. J. Chem. Phys., 103: 8577-8593, 1995. doi: 10.1063/1.470117.

[39] M. Deserno and C. Holm. How to mesh up Ewald sums. I. a theoretical and numerical comparison of various particle mesh routines. J. Chem. Phys., 109:7678-7693, 1998. doi: 10.1063/1.477414.

[40] W. L. Jorgensen, D. S. Maxwell, and J. Tirado-Rives. Development and testing of the OPLS all-atom force field on conformational energetics and properties of organic liquids. J. Am. Chem. Soc, 118:11225-11236, 1996. doi: $10.1021 /$ ja9621760.

[41] A. D. MacKerell et al. All-atom empirical potential for molecular modeling and dynamics studies of proteins. J. Phys. Chem. B, 102:3586-3616, 1998. doi: 10.1021/jp973084f.

[42] L. D. Schuler, X. Daura, and W. F. van Gunsteren. An improved GROMOS96 force field for aliphatic hydrocarbons in the condensed phase. $J$. Comput. Chem., 22:1205-1218, 2001. doi: 10.1002/jcc.1078.

[43] C. Oostenbrink, A. Villa, A. E. Mark, and W. F. van Gunsteren. A biomolecular force field based on the free enthalpy of hydration and solvation: The GROMOS force-field parameter sets 53A5 and 53A6. J. Comput. Chem., 25:1656-1676, 2004. doi: 10.1002/jcc.20090. 
[44] M. P. Allen and D. J. Tildesley. Computer Simulation of Liquids. Clarendon Press, Oxford, 1987.

[45] D. Frenkel and B. Smit. Understanding Molecular Simulation. Academic Press, San Diego, second edition, 2002.

[46] D. C. Rapaport. The Art of Molecular Dynamics Simulation. Cambridge University Press, Cambridge, 2nd edition, 2004.

[47] K. A. Dill, S. Bromberg, K. Z. Yue, K. M. Fiebig, D. P. Yee, P. D. Thomas, and H. S. Chan. Principles of protein-folding - a perspective from simple exact models. Protein Science, 4:561-602, 1995. doi: $10.1002 /$ pro.5560040401.

[48] L. Mirny and E. Shakhnovich. Protein folding theory: From lattice to all-atom models. Ann. Rev. Biophys. Biomol. Struct., 30:361-396, 2001. doi: 10.1146/annurev.biophys.30.1.361.

[49] S. Bandyopadhyay, M. Tarek, and M. L. Klein. Molecular dynamics study of a lipid-dna complex. J. Phys. Chem. B, 103:10075-10080, 1999. doi: 10.1021/jp9927496.

[50] B. L. de Groot and H. Grubmüller. Water permeation across biological membranes: Mechanism and dynamics of aquaporin-1 and GlpF. Science, 294:2353-2357, 2001. doi: 10.1126/science.1066115.

[51] E. Tajkhorshid, P. Nollert, M. O. Jensen, L. J. W. Miercke, J. O'Connell, R. M. Stroud, and K. Schulten. Control of the selectivity of the aquaporin water channel family by global orientational tuning. Science, 296:525530, 2002. doi: 10.1126/science.1067778.

[52] C. F. Lopez, S. O. Nielsen, P. B. Moore, and M. L. Klein. Understanding nature's design for a nanosyringe. Proc. Natl. Acad. Sci. USA, 101:44314434, 2004. doi: 10.1073/pnas.0400352101.

[53] K. V. Damodaran, M. Kenneth, J. Merz, and B. P. Gaber. Structure and dynamics of the dilauroylphosphatidylethanolamine lipid bilayer. Biochemistry, 31:7656-7664, 1992. doi: 10.1021/bi00148a029.

[54] H. Heller, M. Schäfer, and K. Schulten. Molecular dynamics simulation of a bilayer of 200 lipids in the gel and in the liquid crystal phase. $J$. Phys. Chem., 97:8343-8360, 1993. doi: 10.1021/j100133a034.

[55] E. Flenner, J. Das, M. C. Rheinstädter, and I. Kosztin. Subdiffusion and lateral diffusion coefficient of lipid atoms and molecules in phospholipid bilayers. Phys. Rev. E, 79:011907, 2009. doi: 10.1103/PhysRevE.79.011907. 
[56] M. Roark and S. E. Feller. Molecular dynamics simulation study of correlated motions in phospholipid bilayer membranes. J. Phys. Chem. B, 113:13229-13234, 2009. doi: 10.1021/jp902186f.

[57] E. G. Brandt and O. Edholm. Dynamic structure factors from lipid membrane molecular dynamics simulations. Biophys. J., 96:1828-1838, 2009. doi: $10.1016 / j . b p j .2008 .11 .044$.

[58] H. C. Öttinger. Understanding through coarse graining. private communication, October 2009.

[59] J. C. Shelley, M. Y. Shelley, R. C. Reeder, S. Bandyopadhyay, and M. L. Klein. A coarse grain model for phospholipid simulations. J. Phys. Chem. $B, 105: 4464,2001$. doi: 10.1021/jp010238p.

[60] S. J. Marrink, A. H. de Vries, and A. E. Mark. Coarse grained model for semiquantitative lipid simulations. J. Phys. Chem. B, 108:750-760, 2004. doi: 10.1021/jp036508g.

[61] S. O. Nielsen, C. F. Lopez, G. Srinivas, and M. L. Klein. Coarse grain models and the computer simulation of soft materials. J. Phys.: Condens. Matter, 16:R481, 2004. doi: 10.1088/0953-8984/16/15/R03.

[62] R. G. Larson, L. E. Scriven, and H. T. Davis. Monte Carlo simulation of model amphiphile-oil-water systems. J. Chem. Phys., 83:2411-2420, 1985. doi: $10.1063 / 1.449286$.

[63] R. Goetz and R. Lipowsky. Computer simulations of bilayer membranes: self-assembly and interfacial tension. J. Chem. Phys., 108:7397, 1998. doi: $10.1063 / 1.476160$.

[64] H. Noguchi and M. Takasu. Self-assembly of amphiphiles into vesicles: a Brownian dynamics simulation. Phys. Rev. E, 64:041913, 2001. doi: 10.1103/PhysRevE.64.041913.

[65] M. Müller, K. Katsov, and M. Schick. Biological and synthetic membranes: What can be learned from a coarse-grained description? Phys. Rep., 434:113-176, 2006. doi: 10.1016/j.physrep.2006.08.003.

[66] Z.-J. Wang and M. Deserno. A systematically coarse-grained solvent-free model for quantitative phospholipid bilayer simulations. J. Phys. Chem. B, 114:11207-11220, 2010. doi: 10.1021/jp102543j.

[67] S. Izvekov and G. A. Voth. Solvent-free lipid bilayer model using multiscale coarse-graining. J. Phys. Chem. B, 113:4443-4455, 2009. doi: $10.1021 /$ jp 810440 c. 
[68] S. J. Marrink, H. J. Risselada, S. Yefimov, D. P. Tieleman, and A. H. de Vries. The MARTINI force field: Coarse grained model for biomolecular simulations. J. Phys. Chem. B, 111:7812-7824, 2007. doi: 10.1021/jp071097f.

[69] M. Orsi, D. Y. Haubertin, W. E. Sanderson, and J. W. Essex. A quantitative coarse-grain model for lipid bilayers. J. Phys. Chem. B, 112: 802-815, 2008. doi: 10.1021/jp076139e.

[70] O. Farago. Water-free model for fluid bilayer membranes. J. Chem. Phys., 119:596, 2003. doi: 10.1063/1.1578612.

[71] I. R. Cooke, K. Kremer, and M. Deserno. Tunable generic model for fluid bilayer membranes. Phys. Rev. E, 72:011506, 2005. doi: 10.1103/PhysRevE.72.011506.

[72] H. Noguchi and M. Takasu. Structural changes of pulled vesicles: a Brownian dynamics simulation. Phys. Rev. E, 65:051907, 2002. doi: 10.1103/PhysRevE.65.051907.

[73] O. Lenz and F. Schmid. A simple computer model for liquid lipid bilayers. J. Molecular Liquids, 117:147-152, 2005. doi: 10.1016/j.molliq.2004.08.008.

[74] M. J. Stevens. Coarse-grained simulations of lipid bilayers. J. Chem. Phys., 121:11942, 2004. doi: 10.1063/1.1814058.

[75] M. Kranenburg and B. Smit. Phase behavior of model lipid bilayers. J. Phys. Chem. B, 109:6553-6563, 2005. doi: 10.1021/jp0457646.

[76] L. Gao, J. Shillcock, and R. Lipowsky. Improved dissipative particle dynamics simulations of lipid bilayers. J. Chem. Phys., 126:015101, 2007. doi: $10.1063 / 1.2424698$.

[77] A. A. Louis. Beware of density dependent pair potentials. J. Phys.: Condens. Matter, 14:9187-9206, 2002. doi: 10.1088/0953-8984/14/40/311.

[78] G. Brannigan and F. L. H. Brown. Solvent-free simulations of fluid membrane bilayers. J. Chem. Phys., 120:1059-1071, 2004. doi: $10.1063 / 1.1625913$.

[79] G. Brannigan, P. F. Philips, and F. L. H. Brown. Composition dependence of bilayer elasticity. Phys. Rev. E, 72:011915, 2005. doi: 10.1103/PhysRevE.72.011915. 
[80] J. D. Revalee, M. Laradji, and P. B. Sunil Kumar. Implicit-solvent mesoscale model based on soft-core potentials for self-assembled lipid membranes. J. Chem. Phys., 128:035102, 2008. doi: 10.1063/1.2825300.

[81] M. Venturoli, M. M. Sperotto, M. Kranenburg, and B. Smit. Mesoscopic models of biological membranes. Phys. Rep., 437:1-54, 2006. doi: 10.1016/j.physrep.2006.07.006.

[82] S. J. Marrink, A. H. de Vries, and P. Tieleman. Lipids on the move: Simulations of membrane pores, domains, stalks and curves. Biochim. Biophys. Acta Rev. Biomembranes, 1788:149-168, 2008. doi: $10.1016 /$ j.bbamem.2008.10.006.

[83] L. Kramer. Theory of light scattering from fluctuations of membranes and monolayers. J. Chem. Phys., 55:2097-2105, 1971. doi: 10.1063/1.1676380.

[84] U. Seifert and S. A. Langer. Viscous modes of fluid bilayer-membranes. Europhys. Lett, 23:71-76, 1993. doi: 10.1209/0295-5075/23/1/012.

[85] U. Seifert and S. A. Langer. Hydrodynamics of membranes: the bilayer aspect and adhesion. Biophys. Chem., 49:13-22, 1994. doi: 10.1016/0301-4622(93)E0077-I.

[86] H. C. Öttinger. Beyond Equilibrium Thermodynamics. Wiley, Hoboken, 2005.

[87] S. Izvekov and G. A. Voth. Modeling real dynamics in the coarse-grained representation of condensed phase systems. J. Chem. Phys., 125:151101, 2006. doi: $10.1063 / 1.2360580$.

[88] C. Hijón, P. Español, E. Vanden-Eijnden, and R. Delgado-Buscalioni. Mori-Zwanzig formalism as a practical computational tool. Faraday Discussion, 144:301-322, 2010. doi: 10.1039/b902479b.

[89] M. Müller, L. G. MacDowell, P. Virnau, and K. Binder. Interface properties and bubble nucleation in compressible mixtures containing polymers. J. Chem. Phys., 117:5480-5496, 2002. doi: 10.1063/1.1497636.

[90] K. Binder, M. Müller, P. Virnau, and L. G. MacDowell. Polymer plus solvent systems: Phase diagrams, interface free energies, and nucleation. Adv. Polym. Sci, 173:1-104, 2005. doi: 10.1007/b99426.

[91] N. Lefèvre, K. Ch. Daoulas, M. Müller, J.-F. Gohy, and C.-A. Fustin. Selfassembly in thin films of mixtures of block copolymers and homopolymers interacting by hydrogen bonds. Macromolecules, 43:7734-7743, 2010. doi: $10.1021 / \mathrm{ma} 100925 \mathrm{c}$. 
[92] E. Falck, T. Róg, M. Karttunen, and I. Vattulainen. Lateral diffusion in lipid membranes through collective flows. J. Am. Chem. Soc, 130:44-45, 2008. doi: 10.1021/ja7103558.

[93] R. Iftimie, P. Minary, and M. E. Tuckerman. Ab initio molecular dynamics: Concepts, recent developments, and future trends. Proc. Natl. Acad. Sci. USA, 102:6654-6659, 2005. doi: 10.1073/pnas.0500193102.

[94] D. J. Tobias, K. Tu, and M. L. Klein. Atomic-scale molecular dynamics simulations of lipid membranes. Curr. Opin. Coll. Int. Sci., 2:15-26, 1997. doi: 10.1016/S1359-0294(97)80004-0.

[95] H. L. Scott. Modeling the lipid component of membranes. Curr. Opin. Struct. Biol., 12:495-502, 2002. doi: 10.1016/S0959-440X(02)00353-6.

[96] D. Reith, M. Pütz, and F. Müller-Plathe. Deriving effective mesoscale potentials from atomistic simulations. J. Comp. Chem., 24:1624-1636, 2003. doi: 10.1002/jcc.10307.

[97] J. R. Silbermann, S. H. L. Klapp, M. Schoen, N. Chennamsetty, H. Bock, and K. E. Gubbins. Mesoscale modeling of complex binary fluid mixtures: Towards an atomistic foundation of effective potentials. J. Chem. Phys., 124:074105, 2006. doi: 10.1063/1.2161207.

[98] S. Izvekov and G. A. Voth. A multiscale coarse-graining method for biomolecular systems. J. Phys. Chem. B, 109:2469-2473, 2005. doi: 10.1021/jp044629q.

[99] S. Izvekov and G. A. Voth. Multiscale coarse graining of liquid-state systems. J. Chem. Phys., 123:134105, 2005. doi: 10.1063/1.2038787.

[100] W. G. Noid, J. W. Chu, G. S. Ayton, V. Krishna, S. Izvekov, G. A. Voth, A. Das, and H. C. Andersen. The multiscale coarse-graining method. I. a rigorous bridge between atomistic and coarse-grained models. J. Chem. Phys., 128:244114, 2008. doi: 10.1063/1.2938860.

[101] W. G. Noid, P. Liu, Y. Wang, J.-W. Chu, G. S. Ayton, S. Izvekov, H. C. Andersen, and G. A. Voth. The multiscale coarse-graining method. II. numerical implementation for coarse-grained molecular models. J. Chem. Phys., 128:244115, 2008. doi: 10.1063/1.2938857.

[102] P. B. Canham. The minimum energy of bending as a possible explanation of the biconcave shape of the human red blood cell. J. Theor. Biol., 26: 61-81, 1970. doi: 10.1016/S0022-5193(70)80032-7. 
[103] W. Helfrich. Elastic properties of lipid bilayers - theory and possible experiments. Z. Naturforschung C, 28:693-703, 1973.

[104] E. A. Evans. Bending resistance and chemically-induced moments in membrane bilayers. Biophys. J., 14:923-931, 1974. doi: 10.1016/S0006-3495(74)85959-X.

[105] U. Seifert. Configurations of fluid membranes and vesicles. Adv. Phys., 46:13-137, 1997. doi: 10.1080/00018739700101488.

[106] M. Kraus, W. Wintz, U. Seifert, and R. Lipowsky. Fluid vesicles in shear flow. Phys. Rev. Lett., 77:3685-3688, 1996. doi: 10.1103/PhysRevLett.77.3685.

[107] G. Gompper and D. M. Kroll. Network models of fluid, hexatic and polymerized membranes. J. Phys.: Condens. Matter, 9:8795-8834, 1997. doi: $10.1088 / 0953-8984 / 9 / 42 / 001$.

[108] O. G. Mouritsen, A. Boothroyd, R. Harris, N. Jan, T. Lookman, L. MacDonald, D. A. Pink, and M. J. Zuckermann. Computer simulation of the main gel-fluid phase transition of lipid bilayers. J. Chem. Phys., 79: 2027-2041, 1983. doi: 10.1063/1.445987.

[109] J. H. Ipsen, K. Jørgensen, and O. G. Mouritsen. Densityfluctuations in saturated phospholipid-bilayers increase as the acyl-chain length decreases. Biophys. J., 58:1099-1107, 1990. doi: 10.1016/S0006-3495(90)82452-8.

[110] O. G. Mouritsen. Theoretical models of phospholipid phase transitions. Chem. Phys. Lipids, 57:179-194, 1991. doi: 10.1016/0009-3084(91)90075-M.

[111] J. M. Drouffe, A. C. Maggs, and S. Leibler. Computersimulations of self-assembled membranes. Science, 254:1353-1356, 1991. doi: $10.1126 /$ science.1962193.

[112] H. Noguchi. Solvent-free coarse-grained lipid model for large-scale simulations. J. Chem. Phys., 134:055101, 2011. doi: 10.1063/1.3541246.

[113] B. Smit, A. G. Schlijper, L. A. M. Schlijper, and N. M. van Os. Effects of chain length of surfactants on the interfacial tension: molecular dynamics simulations and experiments. J. Phys. Chem., 94:6933-6935, 1990. doi: $10.1021 / \mathrm{j} 100381 \mathrm{a} 003$.

[114] T. Sintes and A. Baumgärtner. Membrane-mediated protein attraction. a Monte-Carlo study. Physica. A, 249:571-575, 1998. doi: 10.1016/S0378-4371(97)00482-2. 
[115] F. Schmid, D. Düchs, O. Lenz, and B. West. A generic model for lipid monolayers, bilayers, and membranes. Comp. Phys. Comm., 177:168171, 2007. doi: 10.1016/j.cpc.2007.02.066.

[116] K. Ch. Daoulas and M. Müller. Comparison of simulations of lipid membranes with membranes of block copolymers. Adv. Polym. Sci, 224:197233, 2009. doi: 10.1007/12_2008_7.

[117] Y. Norizoe, K. Ch. Daoulas, and M. Müller. Measuring excess free energies of self-assembled membrane structures. Faraday Discussion, 144: 363-391, 2010. doi: 10.1039/B901657K.

[118] M. Müller and L. G. MacDowell. Interface and surface properties of short polymers in solution: Monte Carlo simulations and self-consistent field theory. Macromolecules, 33:3902-3923, 2000. doi: 10.1021/ma991796t.

[119] K. G. Soga, M. J. Zuckermann, and H. Guo. Binary polymer brush in a solvent. Macromolecules, 29:1998-2005, 1996. doi: 10.1021/ma951102q.

[120] R. L. Scott and P. H. van Konynenburg. Static properties of solutions. Van der Waals and related models for hydrocarbon mixtures. Discuss. Faraday Soc., 49:87-97, 1970. doi: 10.1039/DF9704900087.

[121] P. H. van Konynenburg and R. L. Scott. Critical lines and phase equilibria in binary Van der Waals mixtures. Phil. Trans. R. Soc. A, 298:495-540, 1980.

[122] M. Müller. Miscibility behavior and single chain properties in polymer blends: a bond fluctuation model study. Macromol. Theory Simul., 8:343-374, 1999. doi: 10.1002/(SICI)1521-3919(19990701)8:4<343::AID-MATS343>3.0.CO;2-F.

[123] T. S. Khasanshin, A. P. Shchamialiou, and O. G. Poddubskij. Thermodynamic properties of heavy n-alkanes in the liquid state: n-dodecane. Int. J. Thermophys., 24:1277-1289, 2003. doi: 10.1023/A:1026199017598.

[124] O. Berger, O. Edholm, and F. Jähnig. Molecular dynamics simulations of a fluid bilayer of dipalmitoylphosphatidylcholine at full hydration, constant pressure, and constant temperature. Biophys. J., 72:2002-2013, 1997. doi: 10.1016/S0006-3495(97)78845-3.

[125] L. F. Braganza and D. L. Worcester. Structural changes in lipid bilayers and biological membranes caused by hydrostatic pressure. Biochemistry, 25:7484-7488, 1986. doi: 10.1021/bi00371a034. 
[126] H. B. Callen. Thermodynamics and an introduction to thermostatistics. John Wiley \& Sons, New York, 1985.

[127] Y. Zhang, S. E. Feller, B. R. Brooks, and R. W. Pastor. Computer simulation of liquid/liquid interfaces. I. theory and application to octane/water. J. Chem. Phys., 103:10252-10266, 1995. doi: 10.1063/1.469927.

[128] W. A. Curtin and N. W. Ashcroft. Weighted-density-functional theory of inhomogeneous liquids and the freezing transition. Phys. Rev. A, 32: 2909-2919, 1985. doi: 10.1103/PhysRevA.32.2909.

[129] F. van Swol and J. R. Henderson. Wetting and drying transitions at a fluid-wall interface. density-functional theory versus computer-simulation. II. Phys. Rev. A, 43:2932-2942, 1991. doi: 10.1103/PhysRevA.43.2932.

[130] A. Yethiraj. Density functional theory of polymers: a Curtin-Ashcroft type weighted density approximation. J. Chem. Phys., 109:3269-3275, 1998. doi: 10.1063/1.476918.

[131] M. Müller, L. G. MacDowell, and A. Yethiraj. Short chains at surfaces and interfaces: a quantitative comparison between density-functional theories and Monte Carlo simulations. J. Chem. Phys., 118:2929-2940, 2003. doi: $10.1063 / 1.1535893$.

[132] J. D. Weeks, K. Katsov, and K. Vollmayr. Roles of repulsive and attractive forces in determining the structure of nonuniform liquids: Generalized mean field theory. Phys. Rev. Lett., 81:4400-4403, 1998. doi: 10.1103/PhysRevLett.81.4400.

[133] K. Katsov and J. D. Weeks. On the mean field treatment of attractive interactions in nonuniform simple fluids. J. Phys. Chem. B, 105:67386744, 2001. doi: 10.1021/jp010893x.

[134] P. Español and P. Warren. Statistical mechanics of dissipative particle dynamics. Europhys. Lett, 30:191-196, 1995. doi: $10.1209 / 0295-5075 / 30 / 4 / 001$.

[135] C. N. Likos, B. M. Mladek, D. Gottwald, and G. Kahl. Why do ultrasoft repulsive particles cluster and crystallize? analytic results from density-functional theory. J. Chem. Phys., 126:224502, 2007. doi: $10.1063 / 1.2738064$.

[136] B. M. Mladek, M. J. Fernaud, G. Kahl, and M. Neumann. On the thermodynamic properties of the generalized Gaussian core model. Condensed Matter Physics, 8:135-148, 2005. 
[137] B. M. Mladek, D. Gottwald, G. Kahl, M. Neumann, and C. N. Likos. Clustering in the absence of attractions: Density functional theory and computer simulations. J. Phys. Chem. B, 111:12799-12808, 2007. doi: $10.1021 / \mathrm{jp} 074652 \mathrm{~m}$.

[138] P. J. Hoogerbrugge and J. M. V. A. Koelman. Simulating microscopic hydrodynamics phenomena with dissipative particle dynamics. Europhys. Lett, 19:155, 1992. doi: 10.1209/0295-5075/19/3/001.

[139] J. M. V. A. Koelman and P. J. Hoogerbrugge. Dynamic simulation of hard-sphere suspensions under steady shear. Europhys. Lett, 21:363, 1993. doi: $10.1209 / 0295-5075 / 21 / 3 / 018$.

[140] R. D. Groot and P. B. Warren. Dissipative particle dynamics: Bridging the gap between atomistic and mesoscopic simulation. J. Chem. Phys., 107:4423-4435, 1997. doi: 10.1063/1.474784.

[141] E. G. Flekkøy and P. V. Coveney. From molecular dynamics to dissipative particle dynamics. Phys. Rev. Lett., 83:1775-1778, 1999. doi: 10.1103/PhysRevLett.83.1775.

[142] I. Pagonabarraga and D. Frenkel. Dissipative particle dynamics for interacting systems. J. Chem. Phys., 115:5015-5026, 2001. doi: $10.1063 / 1.1396848$.

[143] S. Y. Trofimov, E. L. F. Nies, and M. A. J. Michels. Thermodynamic consistency in dissipative particle dynamics simulations of strongly nonideal liquids and liquid mixtures. J. Chem. Phys., 117:9383-9394, 2002. doi: $10.1063 / 1.1515774$.

[144] P. B. Warren. Vapor-liquid coexistence in many-body dissipative particle dynamics. Phys. Rev. E, 68:066702, 2003. doi: 10.1103/PhysRevE.68.066702.

[145] D. van der Spoel, E. Lindahl, B. Hess, G. Groenhof, A.E. Mark, and H. J. C. Berendsen. GROMACS: Fast, flexible, and free. J. Comput. Chem., 26:1701-1718, 2005. doi: 10.1002/jcc.20291.

[146] J. C. Phillips, R. Braun, W. Wang, J. Gumbart, E. Tajkhorshid, E. Villa, C. Chipot, R. Skeel, L. Kale, and K. Schulten. Scalable molecular dynamics with NAMD. J. Comput. Chem., 26:1781-1802, 2005. doi: 10.1002/jcc.20289.

[147] M. Christen, P. H. Hünenberger, D. Barkowies, R. Baron, R. Bürgi, D. P. Geerke, T. N. Heinz, M. A. Kastenholz, V. Kräutler, C. Oostenbrink, C. Peter, D. Trzesniak, and W. F. van Gunsteren. The GROMOS 
software for biomolecular simulation: GROMOS05. J. Comput. Chem., 26:1719-1751, 2005. doi: 10.1002/jcc.20303.

[148] S. J. Plimpton. Fast parallel algorithms for short-range molecular dynamics. J. Comput. Phys., 117:1-19, 1995. doi: 10.1006/jcph.1995.1039.

[149] S. J. Plimpton and B. A. Hendrickson. A new parallel method for molecular-dynamics simulation of macromolecular systems. J. Comp. Chem., 17:326-337, 1996. doi: 10.1002/(SICI)1096-987X(199602)17:3<326::AID-JCC7>3.0.CO;2-X.

[150] V. E. Taylor, R. L. Stevens, and K. E. Arnold. Parallel molecular dynamics: Implications for massively parallel machines. J. Par. Distr. Comput., 45:166-175, 1997. doi: 10.1006/jpdc.1997.1370.

[151] J. W. Shu, B. Wang, M. Chen, J. Z. Wang, and W. M. Zheng. Optimization techniques for parallel force-decomposition algorithm in molecular dynamic simulations. Comp. Phys. Comm., 154:121-130, 2003. doi: 10.1016/S0010-4655(03)00290-X.

[152] G. Sutmann and F. Janoschek. Communication and load balancing of force-decomposition algorithms for parallel molecular dynamics. In C. Bischof, M. Bücker, P. Gibbon, G. R. Joubert, T. Lippert, B. Mohr, and F. Peters, editors, Advances in Parallel Computing, Vol. 15, pages 45-52. IOS Press, Amsterdam, 2008.

[153] D. Brown, J. H. R. Clarke, M. Okuda, and T. Yamazaki. A domain decomposition parallelization strategy for molecular dynamics simulations on distributed memory machines. Comp. Phys. Comm., 74:67-80, 1993. doi: 10.1016/0010-4655(93)90107-N.

[154] M. Pütz and A. Kolb. Optimization techniques for parallel molecular dynamics using domain decomposition. Comp. Phys. Comm., 113:145167, 1998. doi: 10.1016/S0010-4655(98)00074-5.

[155] D. C. Rapaport. Multibillion-atom molecular dynamics simulation: Design considerations for vector-parallel processing. Comp. Phys. Comm., 174:521-529, 2006. doi: 10.1016/j.cpc.2005.11.008.

[156] J. Boris. A vectorized "near neighbors" algorithm of order $\mathrm{n}$ using a monotonic logical grid. J. Comput. Phys., 66:1-20, 1986. doi: 10.1016/0021-9991(86)90050-1.

[157] S. G. Lambrakos and J. P. Boris. Geometric properties of the monotonic lagrangian grid algorithm for near neighbor calculations. J. Comput. Phys., 73:183-202, 1987. doi: 10.1016/0021-9991(87)90113-6. 
[158] R. J. Petrella, I. Andricioaei, B. R. Brooks, and M. Karplus. An improved method for nonbonded list generation: Rapid determination of near-neighbor pairs. J. Comput. Chem., 24:222-231, 2003. doi: $10.1002 /$ jcc.10123.

[159] T. N. Heinz and P. H. Hünenberger. A fast pairlist-construction algorithm for molecular simulations under periodic boundary conditions. $J$. Comput. Chem., 25:1474-1486, 2004. doi: 10.1002/jcc.20071.

[160] T. Maximova and C. Keasar. A novel algorithm for non-bonded-list updating in molecular simulations. J. Comput. Biol., 13:1041-1048, 2006. doi: $10.1089 / \mathrm{cmb} .2006 .13 .1041$.

[161] P. Gonnet. A simple algorithm to accelerate the computation of nonbonded interactions in cell-based molecular dynamics simulations. $J$. Comput. Chem., 28:570-573, 2007. doi: 10.1002/jcc.20563.

[162] S. Meloni, M. Rosati, and L. Colombo. Efficient particle labeling in atomistic simulations. J. Chem. Phys., 126:121102, 2007. doi: $10.1063 / 1.2719690$.

[163] K. Kremer and K. Binder. Monte-Carlo simulations of lattice models for macromolecules. Comp. Phys. Rep., 7:259-310, 1988. doi: 10.1016/0167-7977(88)90015-9.

[164] D. P. Landau and K. Binder. A Guide to Monte Carlo Simulations in Statistical Physics. Cambridge University Press, Cambridge, 2000.

[165] T. Soddemann, B. Dünweg, and K. Kremer. Dissipative particle dynamics: A useful thermostat for equilibrium and nonequilibrium molecular dynamics simulations. Phys. Rev. E, 68:046702, 2003. doi: 10.1103/PhysRevE.68.046702.

[166] B. Dünweg and W. Paul. Brownian dynamics simulations without Gaussian random numbers. Int. J. Mod. Phys. C, 2:817-827, 1991. doi: 10.1142/S0129183191001037.

[167] I. Pagonabarraga, M. H. J. Hagen, and D. Frenkel. Self-consistent dissipative particle dynamics algorithm. Europhys. Lett, 42:377-382, 1998. doi: 10.1209/epl/i1998-00258-6.

[168] G. Besold, I. Vattulainen, M. Karttunen, and J. M. Polson. Towards better integrators for dissipative particle dynamics simulations. Phys. Rev. E, 62:7611-7614, 2000. doi: 10.1103/PhysRevE.62.R7611. 
[169] I. Vattulainen, M. Karttunen, G. Besold, and J. M. Polson. Integration schemes for dissipative particle dynamics simulations: From softly interacting systems towards hybrid models. J. Chem. Phys., 116:3967-3979, 2002. doi: $10.1063 / 1.1450554$.

[170] P. Nikunen, M. Karttunen, and I. Vattulainen. How would you integrate the equations of motion in dissipative particle dynamics simulations? Comp. Phys. Comm., 153:407-423, 2003. doi: 10.1016/S0010-4655(03)00202-9.

[171] C. P. Lowe. An alternative approach to dissipative particle dynamics. Europhys. Lett, 47:145-151, 1999. doi: 10.1209/epl/i1999-00365-x.

[172] C. Junghans, M. Praprotnik, and K. Kremer. Transport properties controlled by a thermostat: An extended dissipative particle dynamics thermostat. Soft Matter, 4:156-161, 2008. doi: 10.1039/b713568h.

[173] M. Tuckerman, B. J. Berne, and G. J. Martyna. Reversible multiple time scale molecular dynamics. J. Chem. Phys., 97:1990-2001, 1992. doi: $10.1063 / 1.463137$.

[174] M. E. Tuckerman and G. J. Martyna. Understanding modern molecular dynamics: Techniques and applications. J. Phys. Chem. B, 104:159-178, 2000. doi: 10.1021/jp992433y.

[175] S. Y. Trofimov, E. L. F. Nies, and M. A. J. Michels. Constant-pressure simulations with dissipative particle dynamics. J. Chem. Phys., 123: 144102, 2005. doi: 10.1063/1.2052667.

[176] A. F. Jakobsen. Constant-pressure and constant-surface tension simulations in dissipative particle dynamics. J. Chem. Phys., 122:124901, 2005. doi: $10.1063 / 1.1867374$.

[177] A. Kolb and B. Dünweg. Optimized constant pressure stochastic dynamics. J. Chem. Phys., 111:4453-4459, 1999. doi: 10.1063/1.479208.

[178] M. Hömberg and M. Müller. Main phase transition in lipid bilayers: Phase coexistence and line tension in a soft, solvent-free, coarse-grained model. J. Chem. Phys., 132:155104, 2010. doi: 10.1063/1.3369005.

[179] J. F. Nagle and S. Tristram-Nagle. Structure of lipid bilayers. Biochim. Biophys. Acta Rev. Biomembranes, 1496:159-195, 2000. doi: 10.1016/S0304-4157(00)00016-2.

[180] M. Kranenburg, M. Venturoli, and B. Smit. Molecular simulations of mesoscopic bilayer phases. Phys. Rev. E, 67:060901(R), 2003. doi: 10.1103/PhysRevE.67.060901. 
[181] I. R. Cooke and M. Deserno. Solvent-free model for self-assembling fluid bilayer membranes: Stabilization of the fluid phase based on broad attractive tail potentials. J. Chem. Phys., 123:224710, 2005. doi: $10.1063 / 1.2135785$.

[182] O. Lenz and F. Schmid. Structure of symmetric and asymmetric "ripple" phases in lipid bilayers. Phys. Rev. Lett., 98:058104, 2007. doi: 10.1103/PhysRevLett.98.058104.

[183] A. H. de Vries, S. Yefimov, A. E. Mark, and S. J. Marrink. Molecular structure of the lecithin ripple phase. Proc. Natl. Acad. Sci. USA, 102: 5392-5396, 2005. doi: 10.1073/pnas.0408249102.

[184] R. M. Hochmuth, N. Mohandas, and Jr P. L. Blackshear. Measurement of the elastic modulus for red cell membrane using a fluid mechanical technique. Biophys. J., 13:747-762, 1973. doi: 10.1016/S0006-3495(73)86021-7.

[185] L. Bo and R. E. Waugh. Determination of bilayer membrane bending stiffness by tether formation from giant, thin-walled vesicles. Biophys. J., 55:509-517, 1989. doi: 10.1016/S0006-3495(89)82844-9.

[186] V. Heinrich and R. E. Waugh. A piconewton force transducer and its application to measurement of the bending stiffness of phospholipid membranes. Annals of Biomedical Engineering, 24:595-605, 1996. doi: $10.1007 / \mathrm{BF} 02684228$.

[187] D. Cuvelier, I. Derényi, P. Bassereau, and P. Nassoy. Coalescence of membrane tethers: Experiments, theory, and applications. Biophys. J., 88:2714-2726, April 2005. doi: 10.1529/biophysj.104.056473.

[188] D. Marsh. Elastic curvature constants of lipid monolayers and bilayers. Chem. Phys. Lipids, 144:146-159, 2006. doi: 10.1016/j.chemphyslip.2006.08.004.

[189] M. Müller and M. Schick. Bulk and interfacial thermodynamics of a symmetric, ternary homopolymer-copolymer mixture: A Monte Carlo study. J. Chem. Phys., 105:8885-8901, 1996. doi: 10.1063/1.472618.

[190] E. S. Boek, J. T. Padding, W. K. den Otter, and W. J. Briels. Mechanical properties of surfactant bilayer membranes from atomistic and coarsegrained molecular dynamics simulations. J. Phys. Chem. B, 109:1985119858, 2005. doi: 10.1021/jp054372b. 
[191] G. Brannigan and F. L. H. Brown. A consistent model for thermal fluctuations and protein-induced deformations in lipid bilayers. Biophys. J., 90:1501-1520, 2006. doi: 10.1529/biophysj.105.075838.

[192] E. Lindahl and O. Edholm. Mesoscopic undulations and thickness fluctuations in lipid bilayers from molecular dynamics simulations. Biophys. $J ., 79: 426,2000$. doi: 10.1016/S0006-3495(00)76304-1.

[193] V. A. Harmandaris and M. Deserno. A novel method for measuring the bending rigidity of model lipid membranes by simulating tethers. $J$. Chem. Phys., 125:204905, 2006. doi: 10.1063/1.2372761.

[194] H. Bermudez, A. K. Brannan, D. A. Hammer, F. S. Bates, and D. E. Discher. Molecular weight dependence of polymersome membrane structure, elasticity, and stability. Macromolecules, 35:8203-8208, 2002. doi: $10.1021 / \mathrm{ma} 0206691$.

[195] H. Bermudez, D. A. Hammer, and D. E. Discher. Effect of bilayer thickness on membrane bending rigidity. Langmuir, 20:540-543, 2004. doi: 10.1021/la035497f.

[196] N. W. Ashcroft and N. D. Mermin. Solid State Physics. Harcourt, Orlando, 1976.

[197] J.-P. Hansen and I. R. McDonald. Theory of Simple Liquids. Academic Press, Amsterdam, 1986.

[198] P. M. Chaikin and T. C. Lubensky. Principles of condensed matter physics. Cambridge University Press, Cambridge, 1995.

[199] H. Ibach and H. Lüth. Festkörperphysik. Springer, Berlin, 2009.

[200] S. A. Safran. Statistical thermodynamics of surfaces, interfaces and membranes. Addison Wesley, Reading, 1994.

[201] W. K. den Otter. Area compressibility and buckling of amphiphilic bilayers in molecular dynamics simulations. J. Chem. Phys., 123:214906, 2005. doi: $10.1063 / 1.2132287$.

[202] M. Kranenburg, J.-P. Nicolas, and B. Smit. Comparison of mesoscopic phospholipid-water models. Phys. Chem. Chem. Phys, 6:4142-4151, 2004. doi: 10.1039/B406433J.

[203] S. Leekumjorn and A. K. Sum. Molecular studies of the gel to liquidcrystalline phase transition for fully hydrated DPPC and DPPE bilayers. Biochim. Biophys. Acta Biomembranes, 1768:354-365, 2006. doi: $10.1016 /$ j.bbamem.2006.11.003. 
[204] T. Heimburg. A model for the lipid pretransition: Coupling of ripple formation with the chain-melting transition. Biophys. J., 78:1154-1165, 2000. doi: 10.1016/S0006-3495(00)76673-2.

[205] M. Nielsen, L. Miao, J. H. Ipsen, M. J. Zuckermann, and O. G. Mouritsen. Off-lattice model for the phase behavior of lipid-cholesterol bilayers. Phys. Rev. E, 59:5790-5803, 1999. doi: 10.1103/PhysRevE.59.5790.

[206] T. C. Lubensky and F. C. MacKintosh. Theory of "ripple" phases of lipid bilayers. Phys. Rev. Lett., 71:1565-1568, 1993. doi: 10.1103/PhysRevLett.71.1565.

[207] C.-M. Chen, T. C. Lubensky, and F. C. MacKintosh. Phase transitions and modulated phases in lipid bilayers. Phys. Rev. E, 51:504-513, 1995. doi: 10.1103/PhysRevE.51.504.

[208] U. S. Schwarz and G. Gompper. Stability of inverse bicontinuous cubic phases in lipid-water mixtures. Phys. Rev. Lett., 85:1472-1475, 2000. doi: 10.1103/PhysRevLett.85.1472.

[209] G. M. Torrie and J. P. Valleau. Nonphysical sampling distributions in Monte Carlo free-energy estimation: Umbrella sampling. J. Comput. Phys., 23:187-199, 1977. doi: 10.1016/0021-9991(77)90121-8.

[210] A. M. Ferrenberg and R. H. Swendsen. Optimized Monte Carlo data analysis. Phys. Rev. Lett., 63:1195-1198, 1989. doi: 10.1103/PhysRevLett.63.1195.

[211] S. Kumar, D. Bouzida, R.H. Swendsen, P.A. Kollman, and J.M. Rosenberg. The weighted histogram analysis method for free-energy calculations on biomolecules. i. the method. J. Comput. Chem., 13:1011-1021, 1992. doi: $10.1002 /$ jcc. 540130812 .

[212] M. Souaille and B. Roux. Extension to the weighted histogram analysis method: combining umbrella sampling with free energy calculations. Comp. Phys. Comm., 135:40-57, 2001. doi: 10.1016/S0010-4655(00)00215-0.

[213] Ch. Chipot and A. Pohorille, editors. Free energy calculations: theory and applications in chemistry and biology. Springer, Berlin, 2007.

[214] W. Shinoda and S. Okazaki. A Voronoi analysis of lipid area fluctuation in a bilayer. J. Chem. Phys., 109:1517-1521, 1998. doi: 10.1063/1.476702.

[215] L. Fousse, G. Hanrot, V. Lefèvre, P. Pélissier, and P. Zimmermann. MPFR: A multiple-precision binary floating-point library with 
correct rounding. ACM Trans. Math. Soft., 33(2):13:1-13:15, 2007. doi: $10.1145 / 1236463.1236468$.

[216] S. L. Veatch, P. Cicuta, P. Sengupta, A. Honerkamp-Smith, D. Holowka, and B. Baird. Critical fluctuations in plasma membrane vesicles. ACS Chem. Biol., 3:287-293, 2008. doi: 10.1021/cb800012x.

[217] J.-M. Allain, C. Storm, A. Roux, M. B. Amar, and J.-F. Joanny. Fission of a multiphase membrane tube. Phys. Rev. Lett., 93:158104, 2004. doi: 10.1103/PhysRevLett.93.158104.

[218] J. de Joannis, F. Y. Jiang, and J. T. Kindt. Coarse-grained model simulations of mixed-lipid systems: Composition and line tension of a stabilized bilayer edge. Langmuir, 22:998-1005, 2006. doi: 10.1021/la051278d.

[219] A. Tian, C. Johnson, W. Wang, and T. Baumgart. Line tension at fluid membrane domain boundaries measured by micropipette aspiration. Phys. Rev. Lett., 98:208102, 2007. doi: 10.1103/PhysRevLett.98.208102.

[220] C. Esposito, A. Tian, S. Melamed, C. Johnson, S.-Y. Tee, and T. Baumgart. Flicker spectroscopy of thermal lipid bilayer domain boundary fluctuations. Biophys. J., 93:3169-3181, 2007. doi: 10.1529/biophysj.107.111922.

[221] S. J. Marrink, J. Risselada, and A. E. Mark. Simulation of gel phase formation and melting in lipid bilayers using a coarse grained model. Chem. Phys. Lipids, 135:223-244, 2005. doi: 10.1016/j.chemphyslip.2005.03.001.

[222] T. Apajalahti, P. Niemelä, P. N. Govindan, M. S. Miettinen, E. Salonen, S.-J. Marrink, and I. Vattulainen. Concerted diffusion of lipids in raft-like membranes. Faraday Discussion, 144:411-430, 2010. doi: $10.1039 / \mathrm{b} 901487 \mathrm{j}$.

[223] K. Simons and E. Ikonen. Functional rafts in cell membranes. Nature, 387:569-572, 1997. doi: 10.1038/42408.

[224] K. Jacobson, O. G. Mouritsen, and R. G. W. Anderson. Lipid rafts: at a crossroad between cell biology and physics. Nature Cell Biol., 9:7-14, 2007. doi: 10.1038/ncb0107-7.

[225] D. P. Kharakoz and E. A. Shlyapnikova. Thermodynamics and kinetics of the early steps of solid-state nucleation in the fluid lipid bilayer. $J$. Phys. Chem. B, 104:10368-10378, 2000. doi: 10.1021/jp001299a. 
[226] K. Binder. Monte Carlo calculation of the surface tension for two- and three-dimensional lattice-gas models. Phys. Rev. A, 25:1699-1709, 1982. doi: 10.1103/PhysRevA.25.1699.

[227] B. A. Berg, U. Hansmann, and T. Neuhaus. Properties of interfaces in the two and three dimensional Ising model. Z. f. Physik B - Condens. Mat., 90:229-239, 1993. doi: 10.1007/BF02198159.

[228] J. E. Hunter and W. P. Reinhardt. Finite-size scaling behavior of the free energy barrier between coexisting phases: Determination of the critical temperature and interfacial tension of the Lennard-Jones fluid. J. Chem. Phys., 103:8627-8637, 1995. doi: 10.1063/1.470121.

[229] K. Binder. Theory of the evaporation/condensation transition of equilibrium droplets in finite volumes. Physica. A, 319:99-114, 2003. doi: 10.1016/S0378-4371(02)01581-9.

[230] L. G. MacDowell, P. Virnau, M. Müller, and K. Binder. The evaporation/condensation transition of liquid droplets. J. Chem. Phys., 120: 5293-5308, 2004. doi: 10.1063/1.1645784.

[231] T. Fischer and R. L. C. Vink. The Widom-Rowlinson mixture on a sphere: elimination of exponential slowing down at first-order phase transitions. J. Phys.: Condens. Matter, 22:104123, 2010. doi: 10.1088/0953-8984/22/10/104123.

[232] M. P. Gelfand and M. E. Fisher. Finite-size effects in fluid interfaces. Physica. A, 166:1-74, 1990. doi: 10.1016/0378-4371(90)90099-E.

[233] E. Chacon and P. Tarazona. Characterization of the intrinsic density profiles for liquid surfaces. J. Phys.: Condens. Matter, 17:S3493-S3498, 2005. doi: $10.1088 / 0953-8984 / 17 / 45 / 039$.

[234] C. Pastorino, K. Binder, and M. Müller. Coarse-grained description of a brush-melt interface in equilibrium and under flow. Macromolecules, 42: 401-410, 2009. doi: 10.1021/ma8015757.

[235] A. Werner, F. Schmid, M. Müller, and K. Binder. "intrinsic" profiles and capillary waves at homopolymer interfaces: A Monte Carlo study. Phys. Rev. E, 59:728-738, 1999. doi: 10.1103/PhysRevE.59.728.

[236] D. S. Dean and R. R. Horgan. Path integrals for stiff polymers applied to membrane physics. Phys. Rev. E, 76:041102, 2007. doi: 10.1103/PhysRevE.76.041102. 
[237] R. Golestanian. Reduced persistence length and fluctuation-induced interactions of directed semiflexible polymers on fluctuating surfaces. Europhys. Lett, 36:557-561, 1996. doi: 10.1209/epl/i1996-00269-3.

[238] J. Tabony and B. Perly. Quasielastic neutron scattering measurements of fast local translational diffusion of lipid molecules in phospholipid bilayers. Biochim. Biophys. Acta Biomembranes, 1063:67-72, 1991. doi: 10.1016/0005-2736(91)90354-B.

[239] S. König, W. Pfeiffer, T. Bayerl, D. Richter, and E. Sackmann. Molecular dynamics of lipid bilayers studied by incoherent quasielastic neutron scattering. J. Phys. II (Paris), 2:1589-1615, 1992. doi: $10.1051 /$ jp2:1992100.

[240] W. L. C. Vaz, R. M. Clegg, and D. Hallmann. Translational diffusion of lipids in liquid crystalline phase phosphatidylcholine multibilayers. a comparison of experiment with theory. Biochemistry, 24:781-786, 1984. doi: $10.1021 /$ bi00324a037.

[241] J. Korlach, P. Schwille, W. W. Webb, and G. W. Feigenson. Characterization of lipid bilayer phases by confocal microscopy and fluorescence correlation spectroscopy. Proc. Natl. Acad. Sci. USA, 96:8461-8466, 1999.

[242] O. Krichevsky and G. Bonnet. Fluorescence correlation spectroscopy: the technique and its applications. Rep. Prog. Phys., 65:251-298, 2002. doi: $10.1088 / 0034-4885 / 65 / 2 / 203$.

[243] P. H. M. Lommerse, H. P. Spaink, and T. Schmidt. In vivo plasma membrane organization: results of biophysical approaches. $B B A$ Biomembranes, 1664:119-131, 2004. doi: 10.1016/j.bbamem.2004.05.005.

[244] N. Kahya, D. Scherfeld, K. Bacia, and P. Schwille. Lipid domain formation and dynamics in giant unilamellar vesicles explored by fluorescence correlation spectroscopy. J. Struct. Biol., 147:77-89, 2004. doi: $10.1016 /$ j.jsb.2003.09.021.

[245] M. H. Cohen and D. Turnbull. Molecular transport in liquids and glasses. J. Chem. Phys., 31:1164-1169, 1959. doi: 10.1063/1.1730566.

[246] H.-J. Galla, W. Hartmann, U. Theilen, and E. Sackmann. On two-dimensional passive random walk in lipid bilayers and fluid pathways in biomembranes. J. Membr. Biol., 48:215-236, 1979. doi: 10.1007/BF01872892. 
[247] W. L. Vaz and P. F. Almeida. Microscopic versus macroscopic diffusion in one-component fluid phase lipid bilayer membranes. Biophys. J., 60: 1553-1554, 1991.

[248] U. Essmann and M. L. Berkowitz. Dynamical properties of phospholipid bilayers from computer simulation. Biophys. J., 76:2081-2089, 1999. doi: 10.1016/S0006-3495(99)77364-9.

[249] E. Lindahl and O. Edholm. Molecular dynamics simulation of NMR relaxation rates and slow dynamics in lipid bilayers. J. Chem. Phys., 115:4938-4950, 2001. doi: 10.1063/1.1389469.

[250] P. B. Moore, C. F. Lopez, and M. L. Klein. Dynamical properties of a hydrated lipid bilayer from a multinanosecond molecular dynamics simulation. Biophys. J., 81:2484-2494, 2001. doi: 10.1016/S0006-3495(01)75894-8.

[251] J. B. Klauda, B. R. Brooks, and R. W. Pastor. Dynamical motions of lipids and a finite size effect in simulations of bilayers. J. Chem. Phys., 125:144710, 2006. doi: 10.1063/1.2354486.

[252] G. S. Ayton and G. A. Voth. Mesoscopic lateral diffusion in lipid bilayers. Biophys. J., 87:3299-3311, 2004. doi: 10.1529/biophysj.104.047811.

[253] W. Kob, C. Donati, S. J. Plimpton, P. H. Poole, and S. C. Glotzer. Dynamical heterogeneities in a supercooled Lennard-Jones liquid. Phys. Rev. Lett., 79:2827-2830, 1997. doi: 10.1103/PhysRevLett.79.2827.

[254] C. Donati, J. F. Douglas, W. Kob, S. J. Plimpton, P. H. Poole, and S. C. Glotzer. Stringlike cooperative motion in a supercooled liquid. Phys. Rev. Lett., 80:2338-2341, 1998. doi: 10.1103/PhysRevLett.80.2338.

[255] E. G. Brandt and O. Edholm. Stretched exponential dynamics in lipid bilayer simulations. J. Chem. Phys., 133:115101, 2010. doi: $10.1063 / 1.3478998$.

[256] M. Roark and S. E. Feller. Molecular dynamics simulation study of correlated motions in phospholipid bilayer membranes. J. Phys. Chem. B, 113:13229-13234, 2009. doi: 10.1021/jp902186f.

[257] S. Busch, C. Smuda, L. C. Pardo, and T. Unruh. Molecular mechanism of long-range diffusion in phospholipid membranes studied by quasielastic neutron scattering. J. Am. Chem. Soc, 132:3232-3233, 2010. doi: $10.1021 /$ ja907581s. 
[258] M. A. Kol, A. I. P. M. de Kroon, D. T. S. Rijkers, J. A. Killian, and B. de Kruijff. Membrane-spanning peptides induce phospholipid flop: A model for phospholipid translocation across the inner membrane of $\mathrm{E}$. coli. Biochemistry, 40:10500-10506, 2001. doi: 10.1021/bi010627+.

[259] M. S. C. Abreu, M. Joao Moreno, and W. L. C. Vaz. Kinetics and thermodynamics of association of a phospholipid derivative with lipid bilayers in liquid-disordered and liquid-ordered phases. Biophys. J., 87: 353-365, 2004. doi: 10.1529/biophysj.104.040576.

[260] J. Liu and J. C. Conboy. 1,2-diacyl-phosphatidylcholine flip-flop measured directly by sum-frequency vibrational spectroscopy. Biophys. J., 89:2522-2532, 2005. doi: 10.1529/biophysj.105.065672.

[261] M. Müller, K. Katsov, and M. Schick. New mechanism of membrane fusion. J. Chem. Phys., 116:2342-2345, 2002. doi: 10.1063/1.1448496.

[262] S. H. Chen, C. Y. Liao, H. W. Huang, T. M. Weiss, M. C. Bellisent-Funel, and F. Sette. Collective dynamics in fully hydrated phospholipid bilayers studied by inelastic x-ray scattering. Phys. Rev. Lett., 86:740-743, 2001. doi: 10.1103/PhysRevLett.86.740.

[263] T. M. Weiss, P.-J. Chen, H. Sinn, E. E. Alp, S.-H. Chen, and H. W. Huang. Collective chain dynamics in lipid bilayers by inelastic X-ray scattering. Biophys. J., 84:3767-3776, 2003. doi: 10.1016/S0006-3495(03)75105-4.

[264] M. C. Rheinstädter, C. Ollinger, G. Fragneto, F. Demmel, and T. Salditt. Collective dynamics of lipid membranes studied by inelastic neutron scattering. Phys. Rev. Lett., 93:108107, 2004. doi: 10.1103/PhysRevLett.93.108107.

[265] M. C. Rheinstädter, J. Das, E. J. Flenner, B. Brüning, T. Seydel, and I. Kosztin. Motional coherence in fluid phospholipid membranes. Phys. Rev. Lett., 101:248106, 2008. doi: 10.1103/PhysRevLett.101.248106.

[266] M. Tarek, D. J. Tobias, S.-H. Chen, and M. L. Klein. Short wavelength collective dynamics in phospholipid bilayers: A molecular dynamics study. Phys. Rev. Lett., 87:238101, 2001. doi: 10.1103/PhysRevLett.87.238101.

[267] J. S. Hub, T. Salditt, M. C. Rheinstädter, and B. L. de Groot. Short-range order and collective dynamics of DMPC bilayers: A comparison between molecular dynamics simulations, X-ray, and neutron scattering experiments. Biophys. J., 93:3156-3168, 2007. doi: $10.1529 /$ biophysj.107.104885. 
[268] A. Einstein. Über die von der molekularkinetischen Theorie der Wärme geforderte Bewegung von in ruhenden Flüssigkeiten suspendierten Teilchen. Ann. Phys. (Leipzig), 17:549-560, 1905.

[269] E. Reister and U. Seifert. Lateral diffusion of a protein on a fluctuating membrane. Europhys. Lett, 71:859-865, 2005. doi: $10.1209 /$ epl/i2005-10139-6.

[270] E. Reister-Gottfried, S. M. Leitenberger, and U. Seifert. Hybrid simulations of lateral diffusion in fluctuating membranes. Phys. Rev. E, 75: 011908, 2007. doi: 10.1103/PhysRevE.75.011908.

[271] A. Rahman. Correlations in the motion of atoms in liquid argon. Phys. Rev., 136:A405-A411, 1964. doi: 10.1103/PhysRev.136.A405.

[272] M. M. Hurley and P. Harrowell. Non-Gaussian behavior and the dynamical complexity of particle motion in a dense two-dimensional liquid. $J$. Chem. Phys., 105:10521-10526, 1996. doi: 10.1063/1.472941.

[273] D. Dubbeldam, D. C. Ford, D. E. Ellis, and R. Q. Snurr. A new perspective on the order- $n$ algorithm for computing correlation functions. Mol. Sim., 35:1084-1097, 2009. doi: 10.1080/08927020902818039.

[274] B. J. Alder and T. E. Wainwright. Velocity autocorrelations for hard spheres. Phys. Rev. Lett., 18:988-990, 1967. doi: 10.1103/PhysRevLett.18.988.

[275] B. J. Alder and T. E. Wainwright. Decay of velocity autocorrelation function. Phys. Rev. A, 1:18-21, 1970. doi: 10.1103/PhysRevA.1.18.

[276] S. C. Glotzer. Spatially heterogeneous dynamics in liquids: insights from simulation. J. Non-Cryst. Solids, 274:342-355, 2000. doi: 10.1016/S0022-3093(00)00225-8.

[277] N. Lačević, F. W. Starr, T. B. Schrøder, and S. C. Glotzer. Spatially heterogeneous dynamics investigated via a time-dependent fourpoint density correlation function. J. Chem. Phys., 119:7372-7387, 2003. doi: $10.1063 / 1.1605094$.

[278] J. Wohlert and O. Edholm. Dynamics in atomistic simulations of phospholipid membranes: Nuclear magnetic resonance relaxation rates and lateral diffusion. J. Chem. Phys., 125:204703, 2006. doi: $10.1063 / 1.2393240$.

[279] M. Doi and S. F. Edwards. The theory of polymer dynamics. Oxford University Press, Oxford, 1986. 
[280] W. K. Kegel and A. van Blaaderen. Direct observation of dynamical heterogeneities in colloidal hard-sphere suspensions. Science, 287:290293, 2000. doi: 10.1126/science.287.5451.290.

[281] E. R. Weeks, J. C. Crocker, A. C. Levitt, A. Schofield, and D. A. Weitz. Three-dimensional direct imaging of structural relaxation near the colloidal glass transition. Science, 287:627-631, 2000. doi: $10.1126 /$ science.287.5453.627.

[282] R. Lipowsky and S. Grotehans. Renormalization of hydration forces by collective protrusion modes. Biophys. Chem., 49:27-37, 1994. doi: 10.1016/0301-4622(93)E0079-K.

[283] F. Brochard and J. F. Lennon. Frequency spectrum of flicker phenomenon in erythrocytes. J. Phys. (Paris), 36:1035-1047, 1975. doi: 10.1051/jphys:0197500360110103500.

[284] P. G. Saffmann and M. Delbrück. Brownian motion in biological membranes. Proc. Natl. Acad. Sci. USA, 72:3111-3113, 1975.

[285] P. G. Saffmann. Brownian motion in thin sheets of viscous fluid. J. Fluid. Mech., 73:593-602, 1976. doi: 10.1017/S0022112076001511.

[286] R. E. Waugh. Surface viscosity measurements from large bilayer vesicle tether formation. II. experiments. Biophys. J., 38:29-37, 1982. doi: 10.1016/S0006-3495(82)84527-X.

[287] K. Velikov, C. Dietrich, A. Hadjiisky, K. Danov, and B. Pouligny. Motion of a massive microsphere bound to a spherical vesicle. Europhys. Lett, 40:405-410, 1997. doi: 10.1209/epl/i1997-00479-1.

[288] R. Dimova, C. Dietrich, A. Hadjiisky, K. Danov, and B. Pouligny. Falling ball viscosimetry of giant vesicle membranes: Finite-size effects. Eur. Phys. J. B, 12:589-598, 1999. doi: 10.1007/s100510051042.

[289] R. Dimova, B. Pouligny, and C. Dietrich. Pretransitional effects in dimyristoylphosphatidylcholine vesicle membranes: Optical dynamometry study. Biophys. J., 79:340-356, 2000. doi: 10.1016/S0006-3495(00)76296-5.

[290] M. J. Saxton and K. Jacobson. Single particle tracking: applications to membrane dynamics. Annu. Rev. Biophys. Biomol. Struct., 26:373-399, 1997.

[291] P. Cicuta, S. K. Keller, and S. L. Veatch. Diffusion of liquid domains in lipid bilayer membranes. J. Phys. Chem. B, 111:3328-3331, 2007. doi: $10.1021 /$ jp0702088. 
[292] R. Dimova, U. Seifert, B. Pouligny, S. Forster, and H. G. Döbereiner. Hyperviscous diblock copolymer vesicles. Eur. Phys. J. E, 7:241-250, 2002. doi: 10.1140/epje/i200101032.

[293] R. Merkel, E. Sackmann, and E. Evans. Molecular friction and epitactic coupling between monolayers in supported bilayers. J. Phys. (Paris), 50: 1535 - 1555, 1989. doi: 10.1051/jphys:0198900500120153500.

[294] R. M. Raphael and R. E. Waugh. Accelerated interleaflet transport of phosphatidylcholine molecules in membranes under deformation. Biophys. J., 71:1374-1388, 1996. doi: 10.1016/S0006-3495(96)79340-2.

[295] A. Yeung and E. Evans. Unexpected dynamics in shape fluctuations of bilayer vesicles. J. Phys. II France, 5:1501-1523, 1995. doi: $10.1051 /$ jp2:1995196.

[296] Y. A. Chizmadzhev, D. A. Kumenko, P. I. Kuzmin, L. V. Chernomordik, J. Zimmerberg, and F. S. Cohen. Lipid flow through fusion pores connecting membranes of different tensions. Biophys. J., 76:2951-2965, 1999. doi: 10.1016/S0006-3495(99)77450-3.

[297] J.-B. Fournier, N. Khalifat, N. Puff, and M. I. Angelova. Chemically triggered ejection of membrane tubules controlled by intermonolayer friction. Phys. Rev. Lett., 102:018102, 2009. doi: 10.1103/PhysRevLett.102.018102.

[298] W. Pfeiffer, S. König, J. F. Legrand, T. Bayerl, D. Richter, and E. Sackmann. Neutron spin echo study of membrane undulations in lipid multibilayers. Europhys. Lett, 23:457-462, 1993.

[299] T. Pott and P. Méléard. The dynamics of vesicle thermal fluctuations is controlled by intermonolayer friction. Europhys. Lett, 59:87-93, 2002. doi: 10.1209/epl/i2002-00163-6.

[300] R. Rodríguez-García, L. R. Arriaga, M. Mell, L. H. Moleiro, I. López-Montero, and F. Monroy. Bimodal spectrum for the curvature fluctuations of bilayer vesicles: Pure bending plus hybrid curvature-dilation modes. Phys. Rev. Lett., 102:128101, 2009. doi: 10.1103/PhysRevLett.102.128101.

[301] S. A. Shkulipa, W. K. den Otter, and W. J. Briels. Thermal undulations of lipid bilayers relax by intermonolayer friction at submicrometer length scales. Phys. Rev. Lett., 96:178302, 2006. doi: 10.1103/PhysRevLett.96.178302. 
[302] S. A. Shkulipa, W. K. den Otter, and W. J. Briels. Surface viscosity, diffusion, and intermonolayer friction: Simulating sheared amphiphilic bilayers. Biophys. J., 89:823-829, 2005. doi: 10.1529/biophysj.105.062653.

[303] S. A. Shkulipa, W. K. den Otter, and W. J. Briels. Simulations of the dynamics of thermal undulations in lipid bilayers in the tensionless state and under stress. J. Chem. Phys., 125:234905, 2006. doi: 10.1063/1.2402919.

[304] W. K. den Otter and S. A. Shkulipa. Intermonolayer friction and surface shear viscosity of lipid bilayer membranes. Biophys. J., 93:423-433, 2007. doi: 10.1529/biophysj.107.105395.

[305] A. W. Lees and S. F. Edwards. The computer study of transport processes under extreme conditions. J. Phys. C, 5:1921-1929, 1972. doi: $10.1088 / 0022-3719 / 5 / 15 / 006$.

[306] A. Khoshnood and G. Gompper H. Noguchi. Lipid membranes with transmembrane proteins in shear flow. J. Chem. Phys., 132:025101, 2010. doi: $10.1063 / 1.3285269$.

[307] F. S. Bates and G. H. Fredrickson. Block copolymer thermodynamics theory and experiment. Ann. Rev. Phys. Chem., 41:525-557, 1990.

[308] P. J. Daivis and D. J. Evans. Comparison of constant pressure and constant volume nonequilibrium simulations of sheared model decane. $J$. Chem. Phys., 100:541-547, 1994. doi: 10.1063/1.466970.

[309] M. Mondello and G. S. Grest. Viscosity calculations of n-alkanes by equilibrium molecular dynamics. J. Chem. Phys., 106:9327-9336, 1997. doi: $10.1063 / 1.474002$.

[310] F. Müller-Plathe. Reversing the perturbation in nonequilibrium molecular dynamics: An easy way to calculate the shear viscosity of fluids. Phys. Rev. E, 59:4894-4898, 1999. doi: 10.1103/PhysRevE.59.4894.

[311] C. M. Tenney and E. J. Maginn. Limitations and recommendations for the calculation of shear viscosity using reverse nonequilibrium molecular dynamics. J. Chem. Phys., 132:014103, 2010. doi: 10.1063/1.3276454.

[312] T. J. Müller and F. Müller-Plathe. Determining the local shear viscosity of a lipid bilayer system by reverse non-equilibrium molecular dynamics simulations. Chem. Phys. Chem., 10:2305-2315, 2009. doi: $10.1002 /$ cphc. 200900156 .

[313] L. Bocquet and J.-L. Barrat. Hydrodynamic boundary-conditions and correlation-functions of confined fluids. Phys. Rev. Lett., 70:2726-2729, 1993. doi: 10.1103/PhysRevLett.70.2726. 
[314] L. Bocquet and J.-L. Barrat. Hydrodynamic boundary-conditions, correlation-functions, and Kubo relations for confined fluids. Phys. Rev. E, 49:3079-3092, 1994. doi: 10.1103/PhysRevE.49.3079.

[315] H. Flyvbjerg and H. G. Petersen. Error estimates on averages of correlated data. J. Chem. Phys., 91:461-466, 1989. doi: 10.1063/1.457480.

[316] B. Liu, J. Goree, and O. S. Vaulina. Test of the Stokes-Einstein relation in a two-dimensional Yukawa liquid. Phys. Rev. Lett., 96:015005, 2006. doi: 10.1103/PhysRevLett.96.015005.

[317] A. Galuschko, L. Spirin, T. Kreer, A. Johner, C. Pastorino, J. Wittmer, and J. Baschnagel. Frictional forces between strongly compressed, nonentangled polymer brushes: Molecular dynamics simulations and scaling theory. Langmuir, 26:6418-6429, 2010. doi: 10.1021/la904119c.

[318] L. Spirin, A. Galuschko, T. Kreer, A. Johner, J. Baschnagel, and K. Binder. Polymer-brush lubrication in the limit of strong compression. Eur. Phys. J. E, 33:307-311, 2010. doi: 10.1140/epje/i2010-10674-3.

[319] J. F. Crilly and J. C. Earnshaw. Photon correlation spectroscopy of bilayer lipid membranes. Biophys. J., 41:197-210, 1983. doi: 10.1016/S0006-3495(83)84420-8.

[320] R. Hirn, R. Benz, and T. M. Bayerl. Collective membrane motions in the mesoscopic range and their modulation by the binding of a monomolecular protein layer of streptavidin studied by dynamic light scattering. Phys. Rev. E, 59:5987-5994, 1999. doi: 10.1103/PhysRevE.59.5987.

[321] J. L. McWhirter, G. Ayton, and G. A. Voth. Coupling field theory with mesoscopic dynamical simulations of multicomponent lipid bilayers. Biophys. J., 87:3242-3263, 2004. doi: 10.1529/biophysj.104.045716.

[322] G. Boedec, M. Leonetti, and M. Jaeger. 3D vesicle dynamics simulations with a linearly triangulated surface. J. Comput. Phys., 230:1020-1034, 2011. doi: $10.1016 /$ j.jcp.2010.10.021.

[323] R. L. McGreevy and L. Pusztai. Reverse Monte Carlo simulation: A new technique for the determination of disordered structures. Mol. Sim., 1: 359-367, 1988. doi: 10.1080/08927028808080958.

[324] A. P. Lyubartsev and A. Laaksonen. Calculation of effective interaction potentials from radial distribution functions: A reverse Monte Carlo approach. Phys. Rev. E, 52:3730-3737, 1995. doi: 10.1103/PhysRevE.52.3730. 
[325] A. P. Lyubartsev, M. Karttunen, P. Vattulainen, and A. Laaksonen. On coarse-graining by the inverse Monte Carlo method: Dissipative particle dynamics simulations made to a precise tool in soft matter modeling. Soft Materials, 1:121-137, 2002. doi: 10.1081/SMTS-120016746.

[326] W. Schommers. Pair potentials in disordered many-particle systems: A study for liquid gallium. Phys. Rev. A, 28:3599-3605, 1983. doi: 10.1103/PhysRevA.28.3599.

[327] F. Müller-Plathe. Coarse-graining in polymer simulation: From the atomistic to the mesoscopic scale and back. Chem. Phys. Chem., 3:754-769, 2002. doi: 10.1002/1439-7641(20020916)3:9<754::AID-CPHC754>3.0.CO;2-U.

[328] M. Praprotnik, L. delle Site, and K. Kremer. Multiscale simulation of soft matter: From scale bridging to adaptive resolution. Ann. Rev. Phys. Chem., 59:545-571, 2008.

[329] N. F. A. van der Vegt, C. Peter, and K. Kremer. Structure-based coarse- and fine-graining in soft matter simulations. In G. A. Voth, editor, Coarse-graining of condensed phase and biomolecular systems. CRC Press, Boca Raton, 2009.

[330] A. Savelyev and G. A. Papoian. Molecular renormalization group coarsegraining of electrolyte solutions: Application to aqueous $\mathrm{NaCl}$ and $\mathrm{KCl}$. J. Phys. Chem. B, 113:7785-7793, 2009. doi: 10.1021/jp9005058.

[331] A. Savelyev and G. A. Papoian. Molecular renormalization group coarsegraining of polymer chains: Application to double-stranded DNA. Biophys. J., 96:4044-4052, 2009. doi: 10.1016/j.bpj.2009.02.067.

[332] T. Murtola, E. Falck, M. Patra, M. Karttunen, and I. Vattulainen. Coarse-grained model for phospholipid/cholesterol bilayer. J. Chem. Phys., 121:9156-9165, 2004. doi: 10.1063/1.1803537.

[333] T. Murtola, E. Falck, M. Karttunen, and I. Vattulainen. Coarsegrained model for phospholipid/cholesterol bilayer employing inverse Monte Carlo with thermodynamic constraints. J. Chem. Phys., 126: 075101, 2007. doi: 10.1063/1.2646614.

[334] W. G. Noid, J.-W. Chu, G. S. Ayton, and G. A. Voth. Multiscale coarsegraining and structural correlations: Connections to liquid-state theory. J. Phys. Chem. B, 111:4116-4127, 2007. doi: 10.1021/jp068549t. 
[335] J. W. Mullinax and W. G. Noid. A generalized-Yvon-Born-Green theory for determining coarse-grained interaction potentials. J. Phys. Chem. C, 114:5661-5674, 2010. doi: 10.1021/jp9073976.

[336] L. Lu and G. A. Voth. Systematic coarse-graining of a multicomponent lipid bilayer. J. Phys. Chem. B, 113:1501-1510, 2009. doi: $10.1021 / j p 809604 \mathrm{k}$.

[337] S. Izvekov and G. A. Voth. Multiscale coarse-graining of mixed phospholipid/cholesterol bilayers. J. Chem. Theo. Comp., 2:637-648, 2006. doi: 10.1021/ct050300c.

[338] G. S. Ayton and G. A. Voth. Hybrid coarse-graining approach for lipid bilayers at large length and time scales. J. Phys. Chem. B, 113:44134424, 2009. doi: 10.1021/jp8087868.

[339] C. A. Marsh, G. Backx, and M. H. Ernst. Fokker-Planck-Boltzmann equation for dissipative particle dynamics. Europhys. Lett, 38:411-415, 1997. doi: 10.1209/epl/i1997-00260-6.

[340] P. Kindt and W. J. Briels. Scaling of mesoscale simulations of polymer melts with the bare friction coefficient. J. Chem. Phys., 123:224903, 2005. doi: $10.1063 / 1.2132284$.

[341] F. Lahmar and B. Rousseau. Influence of the adjustable parameters of the DPD on the global and local dynamics of a polymer melt. Polymer, 48:3584-3592, 2007. doi: 10.1016/j.polymer.2007.04.018.

[342] C. de Boor. A Practical Guide to Splines. Springer, Berlin, 2001.

[343] J. D. Weeks, D. Chandler, and H. C. Andersen. Role of repulsive forces in determining the equilibrium structure of simple liquids. J. Chem. Phys., 54:5237-5247, 1971. doi: 10.1063/1.1674820.

[344] H. E. Stanley. Introduction to Phase Transitions and Critical Phenomena. Clarendon Press, Oxford, 1971. 



\section{Curriculum Vitae}

\section{Persönliche Daten}

Name: Martin Hömberg

Geburtstag, -ort: 24.08.1981 in Wesel

Adresse: $\quad$ Ginsterweg 4, 37077 Göttingen

Nationalität: deutsch

Familienstand: ledig

Konfession: röm. katholisch

\section{Ausbildung}

$1988-1992$

Theodor-Heuss-Grundschule Wesel-Flüren

$1992-2001$

Konrad-Duden-Gymnasium Wesel

Juni 2001

Allgemeine Hochschulreife, Note: 1,3

$2001-2002$

Zivildienst am Marien-Hospital Wesel

\section{Studium}

Oktober 2002

Beginn Studium Physik an der Uni Göttingen

Oktober 2004

Vordiplom Physik, Note: sehr gut

Juli 2007

Diplom Physik, Note: mit Auszeichnung

September 2007

Beginn Promotion Physik mit Prof. M. Müller

am Institut für Theor. Physik der Uni Göttingen 



\section{Liste der Veröffentlichungen}

1. M. Hömberg, „Ein Verfahren zur schnellen Einführung von intermolekularen Korrelationen in Homopolymere", Diplomarbeit (2007)

2. M. Hömberg, M. Müller, "Generating multi-chain configurations of an inhomogeneous melt from the knowledge of single-chain properties", J. Chem. Phys. 128, 224911 (2008)

3. M. Hömberg, M. Müller, „Main phase transition in lipid bilayers: phase coexistence and line tension in a soft, solvent-free, coarse-grained model", J. Chem. Phys. 132, 155104 (2010) 